\title{
Mitochondrial adaptation at the neuronal presynapse
}

\author{
Dissertation \\ for the award of the degree \\ "Doctor rerum naturalium" (Dr.rer.nat.) \\ Division of Mathematics and Natural Sciences \\ of the Georg August University Göttingen \\ within the doctoral program Molecular Medicine \\ of the Georg-August University School of Science (GAUSS)
}

submitted by Felix Lange

from Leipzig

Göttingen, 2020 


\section{Thesis Committee}

Prof. Dr. Stefan Jakobs

Department of NanoBiophotonics

Mitochondrial Structure and Dynamics Group

Max Planck Institute for Biophysical Chemistry, Göttingen

Prof. Dr. Peter Rehling

Department of Cellular Biochemistry

University Medical Center Göttingen

Prof. Dr. Reinhard Jahn

Department of Neurobiology

Max Planck Institute for Biophysical Chemistry, Göttingen

\section{Members of the Examination Board}

Reviewer:

Prof. Dr. Stefan Jakobs

Second Reviewer: $\quad$ Prof. Dr. Peter Rehling

\section{Further members of the Examination Board:}

Prof. Dr. Reinhard Jahn

Prof. Dr. Silvio Rizzoli

Department of Neuro- and Sensory Physiology

University Medical Center Göttingen

Prof. Dr. Carolin Wichmann

Institute for Auditory Neuroscience

University Medical Center Göttingen

Prof. Dr. Tiago Fleming Outeiro

Department of Experimental Neurodegeneration

University Medical Center Göttingen

Date of the oral examination: 25.02 .2021 


\section{Affidavit}

I hereby declare, that I prepared the $\mathrm{PhD}$ thesis entitled:

\section{"Mitochondrial adaptation at the neuronal presynapse"}

That was submitted on the $30^{\text {th }}$ of December, 2020 is the result of my own work and prepared with no other sources than quoted.

Göttingen, 30 ${ }^{\text {th }}$ of December 2020

Felix Lange 


\section{Summary}

Synaptic transmission poses a major energy consuming process in the brain, but how neurons maintain a constant energy supply during extended synaptic activity and how presynaptic mitochondria contribute to the energy supply remains elusive. The mitochondria are key organelles to account for the majority of ATP newly synthesised in neuronal cells. Hence, the present study aimed at unravelling the structural adaptation of mitochondria to an increase in presynaptic energy demand. To this end, I first characterized the mitochondrial membrane architecture and how it changes in response to different energy sources using transmission electron microscopy. As a model system, several cancer cell lines were used in addition to primary hippocampal neuronal cultures isolated from rat brain. In all tested cancer cell lines, a long-term metabolic switch to ketosis induced significant changes in the mitochondrial structure reflected by an increase of the mitochondrial diameter as well as a significant increase in the abundance of crista membranes. In contrast, a metabolic switch to glycolysis resulted in a decrease of the mitochondrial diameter and a reorientation of the crista membranes in parallel to the mitochondrial outer membrane in these cells. Likewise, in cultivated hippocampal neurons, the metabolic switch to ketosis induced a $20 \%$ increase of crista membranes. Interestingly, only about $35 \%$ of the neuronal presynapses showed a mitochondrial occupation which was not influenced by chemical depolarization with high concentrations of potassium chloride $(\mathrm{KCl})$, suggesting that induced presynaptic activity does not affect the travelling of axonal mitochondria to the presynapses of hippocampal neurons.

Furthermore, I used the cochlear nucleus of mice before and after the onset of hearing as a model system for presynaptic states of low and high-energy demand. Here, the synaptic morphology together with the presynaptic mitochondrial structure using Focused lon Beam Scanning Electron Microscopy (FIB-SEM) was assessed. The volume of the synaptic boutons, the synaptic vesicle pool as well as the post-synaptic density were increased in synapses after the onset of hearing. Beyond that, the mitochondrial volume in these presynapses increased significantly. This suggests that synapses and mitochondria undergo major structural changes to serve the higher energy demands after the onset of hearing. Intriguingly, synapses containing mitochondria displayed an overall larger volume, synaptic vesicle pool and postsynaptic density when compared to synapses lacking mitochondria in mice before and after the onset of hearing. In conclusion, mitochondria play a key role in shaping presynaptic structure and are thus pivotal for synaptic transmission.

In order to gain functional insights into the role of mitochondria in synaptic transmission in addition to the structural information obtained by TEM, I developed novel correlative imaging 
tools. These tools include live cell 3D correlative light and electron microscopy (3D CLEM), which allows to monitor relevant mitochondrial targets by fluorescent labelling and subsequently correlate the functional information with structural aspects, and high-accuracy CLEM allowing for high spatial resolution of the detected features in both, light and electron microscopy.

These protocols built the foundation for an extended correlative approach. There, I combined high-accuracy CLEM with nanoscale secondary ion mass spectrometry (NanoSIMS). This, for the first time, allowed extracting the functional and structural information together with the chemical composition of subcellular areas of the same resin-embedded specimen. These tools could now be applied to study individual players in mitochondrial function by simultaneously getting information of their impact on synaptic structure and chemical composition. 


\section{List of Figures}

Figure 1: Synaptic vesicle cycle at the neuronal presynapse. .................................... 16

Figure 2: Energy consuming processes at the neuronal synapse.................................. 17

Figure 3: Mitochondria in HeLa cells. ...................................................................... 19

Figure 4: Transmission electron micrographs showing the mitochondrial structure in cultivated rat hippocampal neurons.

Figure 5: The ultrastructure of a HeLa wildtyp cell visualized by electron microscopy. 24

Figure 6: Sample preparation routes of biological specimens for correlative light and electron microscopy.

Figure 7: Live cell CLEM enables identification of targets. 28

Figure 8: Correlative light and electron microscopy of HeLa cells expressing a mitochondria targeted mCitrine.

Figure 9: Transmission electron microscopy images of rat hippocampal neurons prepared with the standard protocol.

Figure 10: Transmission electron micrographs of rat hippocampal neurons and HeLa cells suing the high-contrast protocol.

Figure 11: fBROPA protocol preparations of rat hippocampal neurons and HeLa cells. 44

Figure 12: Adapted high contrast protocol preparations of rat hippocampal neurons and HeLa cells. 46

Figure 13: Transmission electron microscopy images of mitochondria from HeLa, U-2 OS and Cos7 cells.

Figure 14: Transmission electron micrographs of HeLa, U-2 OS and Cos7 cells grown in SILAC FLEX with dialyzed serum and without glutamine.

Figure 15: Morphological adaptations of mitochondria to a specific energy substrate in HeLa, Cos7 and U-2 OS cells. 52

Figure 16: Average crista length per area of mitochondrial cross-section. 53

Figure 17: The Metabolic switch changes the length of crista membranes [ $\mu \mathrm{m}]$ per $\mu \mathrm{m}$ of mitochondrial length. 55

Figure 18: Mitochondrial occupation of presynapses in cultured rat hippocampal neurons. 57 
Figure 19: Transmission electron microscopy of the mitochondrial inner membrane architecture at different neuronal sites. 58

Figure 20: Comparison of mitochondria across model systems. 59

Figure 21: Exemplary transmission electron micrographs of mitochondria in hippocampal neurons grown in presence of $25 \mathrm{mM}$ beta-hydroxybutyrate. 60

Figure 22: Exemplary transmission electron micrographs of presynaptic mitochondria of neurons after cultivation for 21 DiV.

Figure 23: Morphological measurements in presynaptic mitochondria of rat hippocampal neurons after 21 DiV. 62

Figure 24: Structural analysis of neuronal presynapses in-vivo. 65

Figure 25: Quantification of the structural details of presynapses identified in a P9 animal as well as in a P20 animal. 66

Figure 26: Development of substrates suitable for correlative imaging. 68

Figure 27: Live cell CLEM of EGFP-Bax expressing HeLa-cells. 71

Figure 28: 3D-CLEM of mitochondria during apoptosis. 72

Figure 29: 3D reconstruction of apoptotic mitochondria as identified by CLEM. 73

Figure 30: Temporal succession of Bax-mediated apoptosis in HeLa cells analysed with transmission electron microscopy. 74

Figure 31: High-accuracy CLEM. 77

Figure 32: High-accuracy CLEM in detailed view. 78

Figure 33: Transmission electron microscopy correlated to NanoSIMS... 80

Figure 34: CLEM-SIMS of HeLa cells. 82

Figure 35: Mitochondrial architecture of cancer cells depending on the available carbon substrate. 86

Figure 36: Representative electron micrographs of neuronal mitochondria in hippocampal brain slices.

Figure 37: Ultrastructure of axonal mitochondria. 88

Figure 38: Structural heterogeneity of neuronal mitochondria depending on their positioning in the cells. 
Figure 39: Structural adaptations of presynapses in the cochlear nucleus after maturation and development. 94

Figure 40: Mitochondrial membrane morphology in HeLa cells during intrinsic apoptosis. 100 Figure 41: Samples for correlative light and electron microscopy

Figure 42: 3D CLEM of Bax-EGFP. 120

Figure 43: Statistical tests for changes of the mitochondrial diameter..... 121

Figure 44: Statistical test for changes in the average crista membrane abundance per mitochondrial area 122

Figure 45: Statistical test for changes in the average crista membrane abundance per mitochondrial length.

Figure 46: Statistical tests for mitochondrial adaptation in cultivated rat hippocampal neurons.

Figure 47: Box and whisker plots of features identified in FIB-SEM micrographs of the cochlear nucleus of $\mathrm{P} P 9$ and a $P 20$ animal. 125

Figure 48: Statistical tests for structural features identified in FIB-SEM micrographs..... 127 


\section{List of Tables}

Table 1: Advantages and downsides of sample preparation protocols for transmission electron microscopy of neuronal mitochondria in the soma........................................................ 47

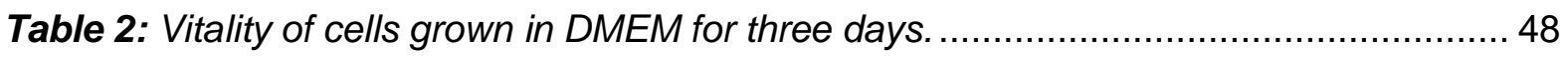

Table 3: Vitality of cells grown in SILAC FLEX medium without glutamine.........................50 


\section{Contents}

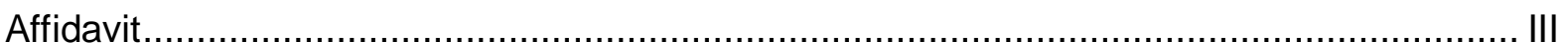

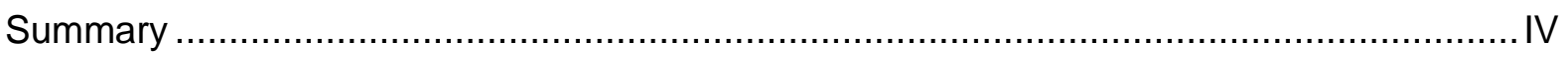

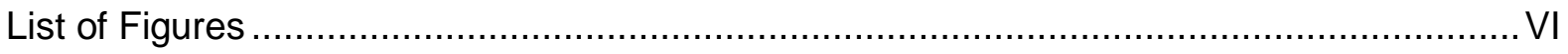

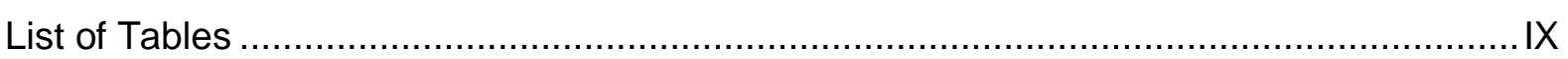

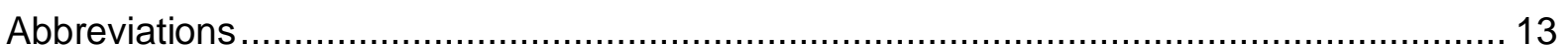

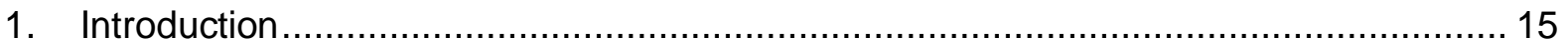

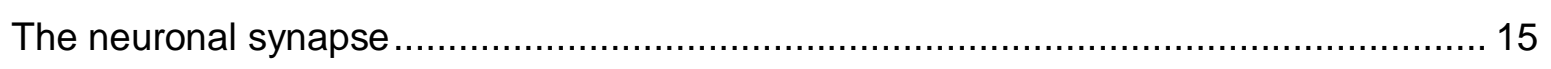

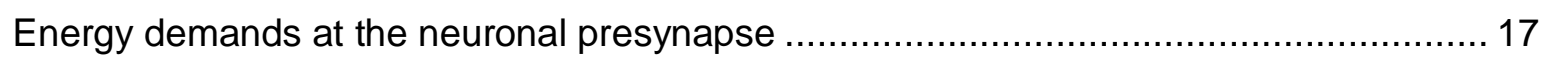

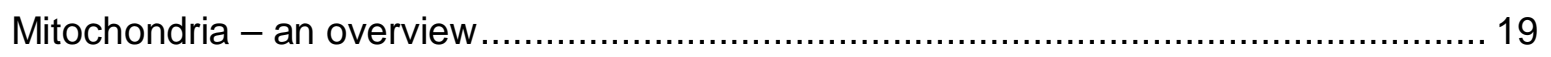

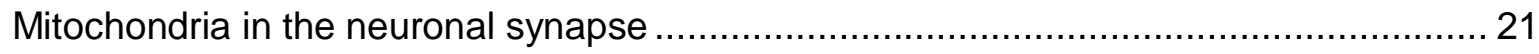

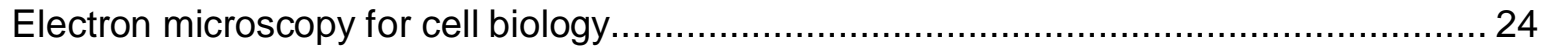

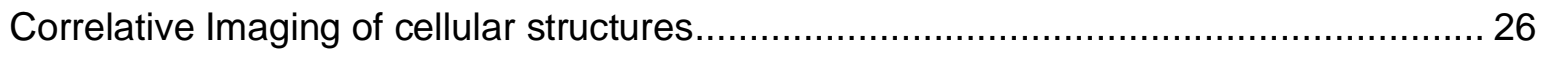

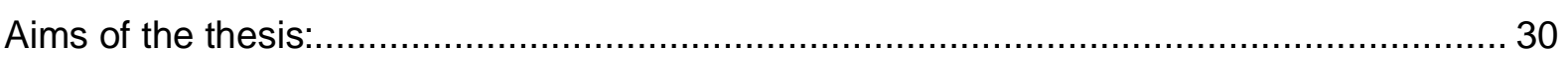

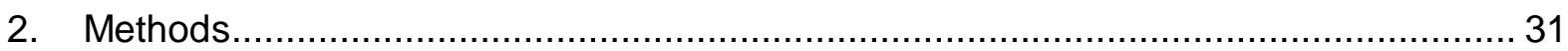

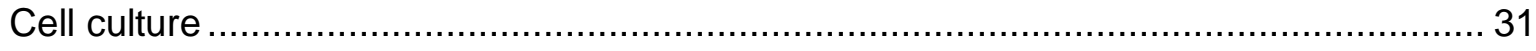

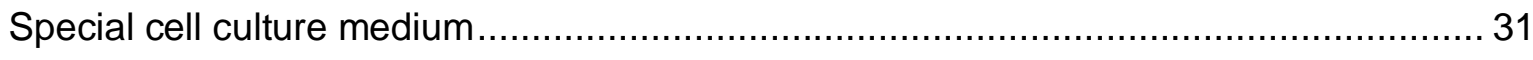

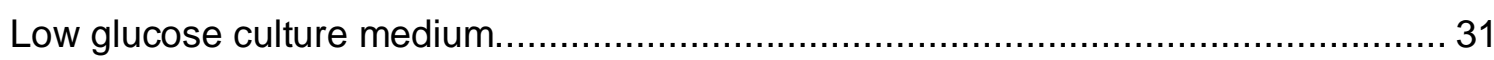

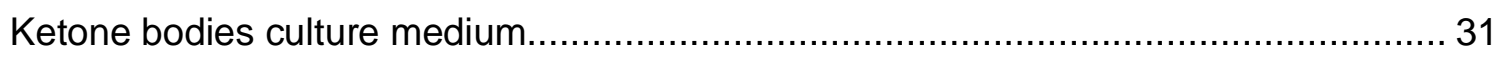

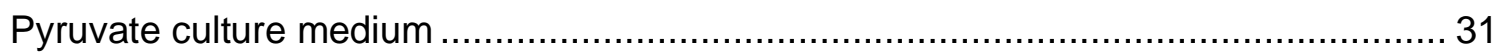

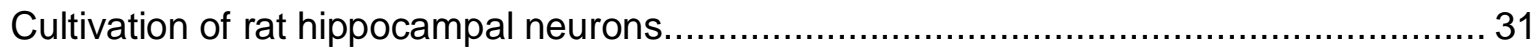

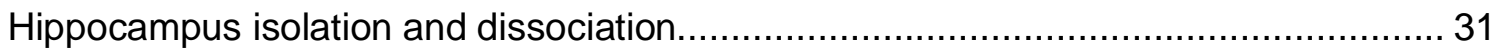

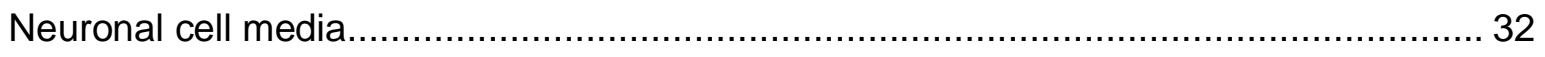

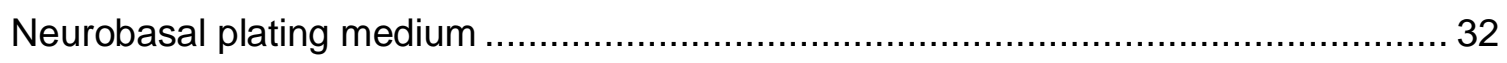

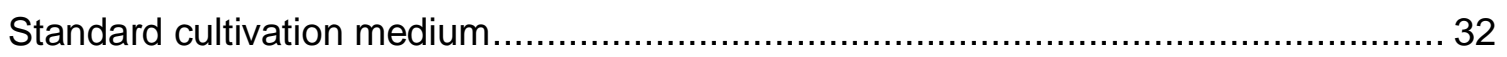

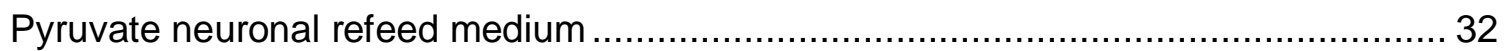

Ketone bodies neuronal refeed medium............................................................. 33

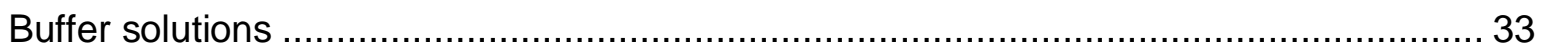

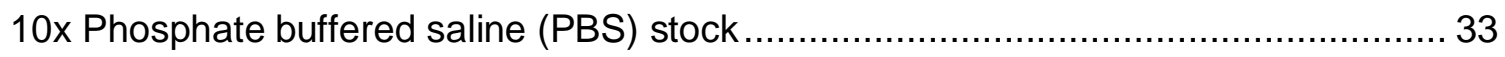

Phosphate buffered saline working solutions ......................................................... 33

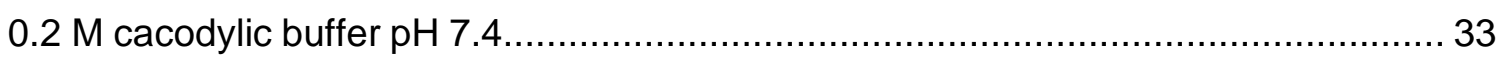

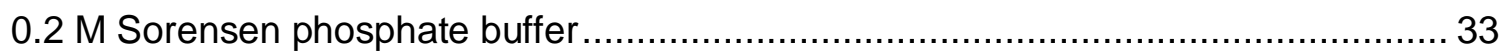

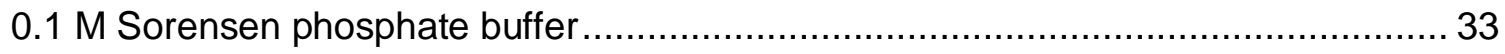

Sample preparations of cells for transmission electron microscopy (TEM) ….................. 34 


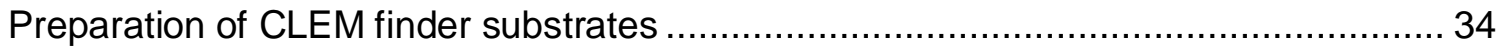

Cell immobilization for chemical aldehyde fixation (CAF) ...................................... 34

Standard staining procedure for electron microscopy............................................... 34

High membrane contrast procedure for electron microscopy .................................... 35

fBROPA protocol for serial blockface SEM imaging ................................................. 35

Adapted fBROPA protocol for transmission electron microscopy ............................... 36

Cell immobilization for high-pressure frozen cells .................................................... 37

Freeze substitution and resin embedding for CLEM.............................................. 37

High-accuracy post-embedding CLEM......................................................... 38

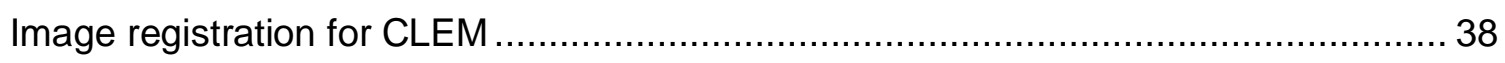

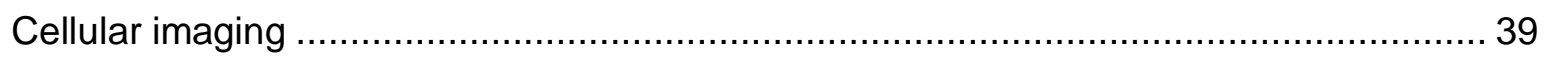

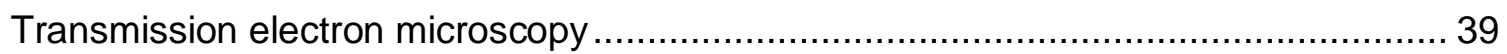

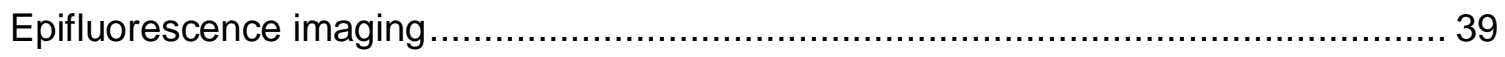

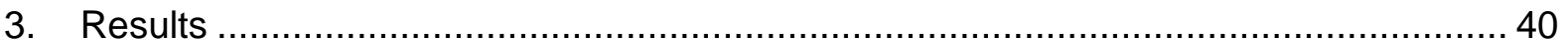

Section 1 - Mitochondrial adaptation in the neuronal presynapse .............................. 40

Development of high contrast staining procedures for electron microscopy .................40

High contrast membrane procedure ........................................................................ 41

fBROPA protocol for enhanced membrane staining .................................................. 43

Adapted high contrast protocol for neuronal cells.................................................. 45

Preliminary experiments towards mitochondrial adaptation during metabolic switch........ 47

Structural adaptations of mitochondria in presence of different carbon substrates in cultivated cancer cell lines .............................................................................. 48

Structural adaptations of mitochondria in presence of different carbon substrates in cultivated cancer cell lines in specialized medium............................................... 50

Mitochondrial occupation of rat hippocampal presynapses .................................... 56

Cultivation of rat hippocampal neurons in presence of different carbon substrates ...... 58

Mitochondrial inner membrane architecture after chemical depolarization ...................61 61

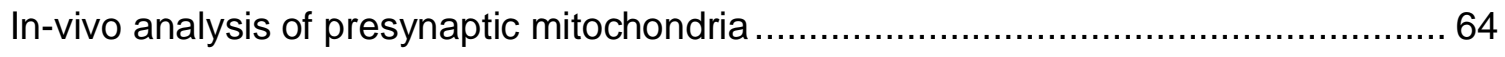

Section 2 - Protocol establishments for correlative imaging of cells ........................... 68

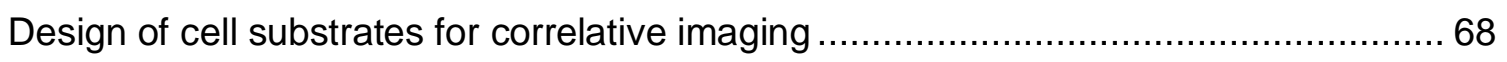

Live cell CLEM of Bax-EGFP expressing cells ................................................... 69

The temporal succession of BAX mediated apoptosis......................................... 74

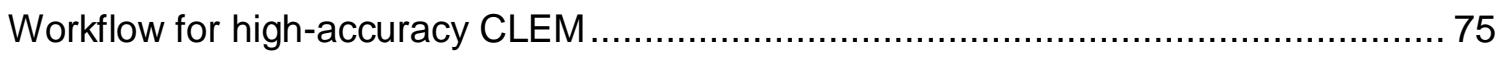

Expanding the information obtained by correlative imaging .................................... 79

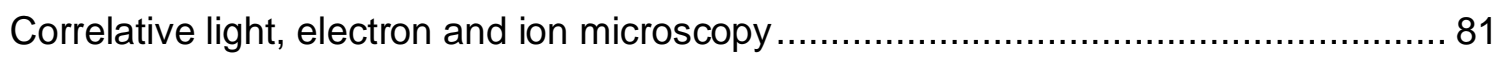

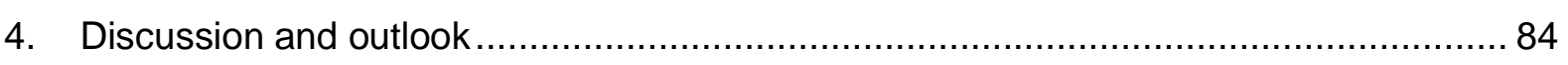

Section 1 - Mitochondrial adaptation in the neuronal presynapse ................................... 84 
Influence of the carbon source on mitochondrial membrane ultrastructure in cancer cells

Establishment of a staining protocol for neuronal mitochondria in electron microscopy 87

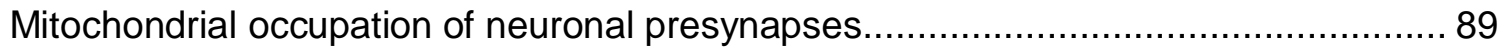

Effects of carbon substrate availability on mitochondrial structure in neuronal cells .....90

Chemical depolarization of neuronal cells ............................................................ 92

Mitochondrial adaptation in presynapses of the cochlear nucleus in mice....................93

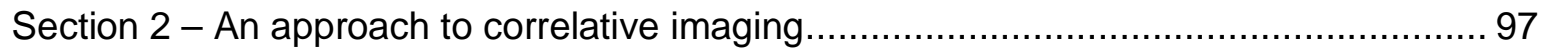

Design of CLEM-compatible cell substrates.......................................................... 97

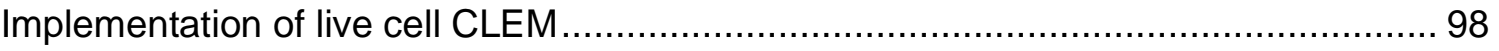

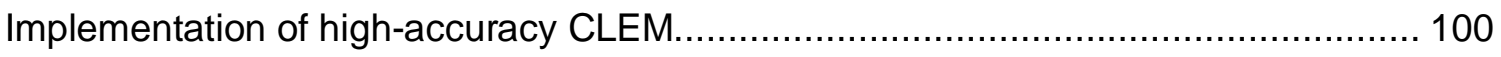

Expanding the information obtained by correlative imaging................................ 101

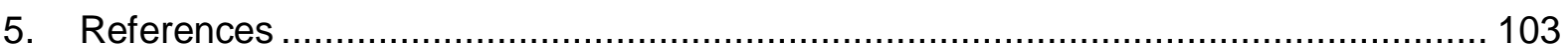

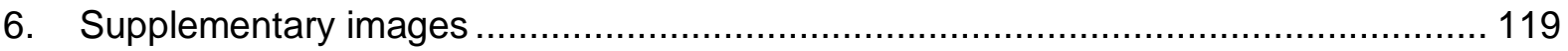

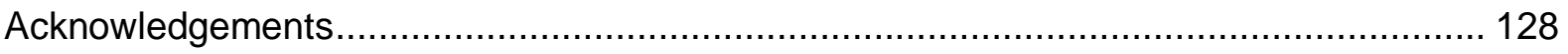

CV

Error! Bookmark not defined. 


\section{Abbreviations}

\begin{tabular}{|c|c|}
\hline ATP & Adenosine IriPhosphate \\
\hline $\mathrm{nm}$ & nanometres \\
\hline OxPhos & $\underline{\text { Oxidative Phosphorylation }}$ \\
\hline CLEM & Correlative Light and Electron Microscopy \\
\hline HPF & High-Pressure Freezing \\
\hline TEM & Transmission Electron Microscopy \\
\hline SEM & Scanning Electron Microscopy \\
\hline DiV & Days in $\underline{\text { Vitro }}$ \\
\hline FIB & Focussed lon Beam \\
\hline LM & Light Microscopy \\
\hline fBROPA & $\begin{array}{l}\text { fast } \underline{B} \text { rain-wide } \underline{\text { Reduced-O}} \text { smium staining with } \underline{\text { Pyrogallol-mediated }} \\
\text { Amplification }\end{array}$ \\
\hline$P$ & Postnatal day \\
\hline DMEM & Dulbecco's Modified Eagale Medium \\
\hline HBSS & Hank's B Buffered Salt Solution \\
\hline bHB & $\underline{b} e t a-\underline{H} y d r o x y \underline{B} u t y r a t e$ \\
\hline NanoSIMS & Nanoscale Secondary Ion Mass Spectrometry \\
\hline PBS & Phosphate Buffered Saline \\
\hline DAPI & 4',6-ㅁiAmidino-2-ㅁhenyllndole \\
\hline $2 \mathrm{D} / 3 \mathrm{D}$ & two dimensional/three dimensional \\
\hline GFP & Green Fluorescent Protein \\
\hline CAF & Classical Aldehyde Fixation \\
\hline SPB & Sorensens Phosphate Buffer \\
\hline ssTEM & serial sectioning Iransmission Electron Microscopy \\
\hline CME & Clathrin Mediated Endocytosis \\
\hline
\end{tabular}


SVs $\quad$ Synaptic Vesicles

OMM $\quad \underline{\text { Outer Mitochondrial Membrane }}$

IBM Inner Boundary Membrane

CM $\quad$ Crista Membrane

ETC Electron Transport $\underline{\text { Chain }}$

TCA $\quad$ TriCharboxylic $\underline{\text { Acid }}$

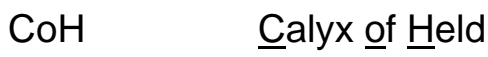

$\mathrm{KCl} \quad$ Potassium chloride 


\section{Introduction}

\section{The neuronal synapse}

Neurons are highly specialized cells in the mammalian brain. Their primary function is the receiving and transmittance of information. The cell body of neuronal cells is called the soma. Further, neuronal cells have functionally different sites that are located multiple micrometres away from the actual cell body. Dendrites are elongated tree-like cell extensions proceeding the soma. These cell parts are required to receive and process signals from other neuronal cells. Postsynapses for example are important structures that are located in the dendrites. The specialized projection that carries the signal away from the cell body is called the axon. The axon forms presynaptic boutons and presynaptic terminals.

The intercellular transmission of signals between neuronal cells is enabled by synaptic connections. A synaptic connection consists of a presynapse with an associated postsynapse. These two compartments communicate by means of electrical and chemical stimuli (1-3). The chemical messengers are termed neurotransmitters. Neurotransmitter in the central nervous system include small molecules like for example glutamate, GABA, serotonin or dopamine (46). Prior to release, these molecules are stored in small vesicular compartments called synaptic vesicles.

Synaptic vesicles at presynapses number between several dozen and a thousand. The abundance of vesicles in a presynaptic site is an important characterisation for the functional state of that synapse. The majority of the vesicles is scattered across the bouton volume and accumulating evidence suggests that these vesicles are organized in a filamentous protein matrix $(7,8)$. A smaller number of vesicles, termed docked vesicles, is located in close proximity to a protein-rich are of the plasma membrane that is called the active zone (9).

At the active zone, the fusion of synaptic vesicles occurs. The fusion of synaptic vesicles ultimately leads to the release of stored neurotransmitters into the extracellular space that is called the synaptic cleft (10). The synaptic cleft is defined as the gap between presynapse and postsynapse that is about $20 \mathrm{~nm}$ wide (11). Located on the other side of the synaptic cleft is the postsynapse that harbours receptors for neurotransmitters.

For glutamate receptors for example it was demonstrated, that the spatial organization of the receptor molecules with respect to the presynaptic release site mediate fast or long-lasting transmission signals (12). Scaffolding proteins that mediate downstream signalling of the action potential are located beneath the postsynaptic plasma membrane forming the 
postsynaptic density that can be visualized as an electron-dense layer in electron microscopy $(13,14)$.

The membrane potential of neuronal cells is negative compared to the extracellular space. The depolarization mediated by sodium influx through voltage-gated sodium channels is called an action potential. The depolarization in turn, opens voltage-gated calcium ions located in the membrane of presynaptic boutons. An increase of intracellular calcium concentrations in the presynapse stimulates the membrane fusion of synaptic vesicles at the active zone $(15,16)$ (Figure 1). Key players of this membrane fusion machinery include synaptotagmin localized on synaptic vesicles that can promote interactions to SNARE proteins that mediate membrane fusion $(15,17,18)$. Sustained elevated levels of calcium ions at the presynapse can lead to continuous release of neurotransmitter filled synaptic vesicles. The clearance of presynaptic calcium ions is a crucial mechanism to control synaptic strength on the presynaptic site (1922).

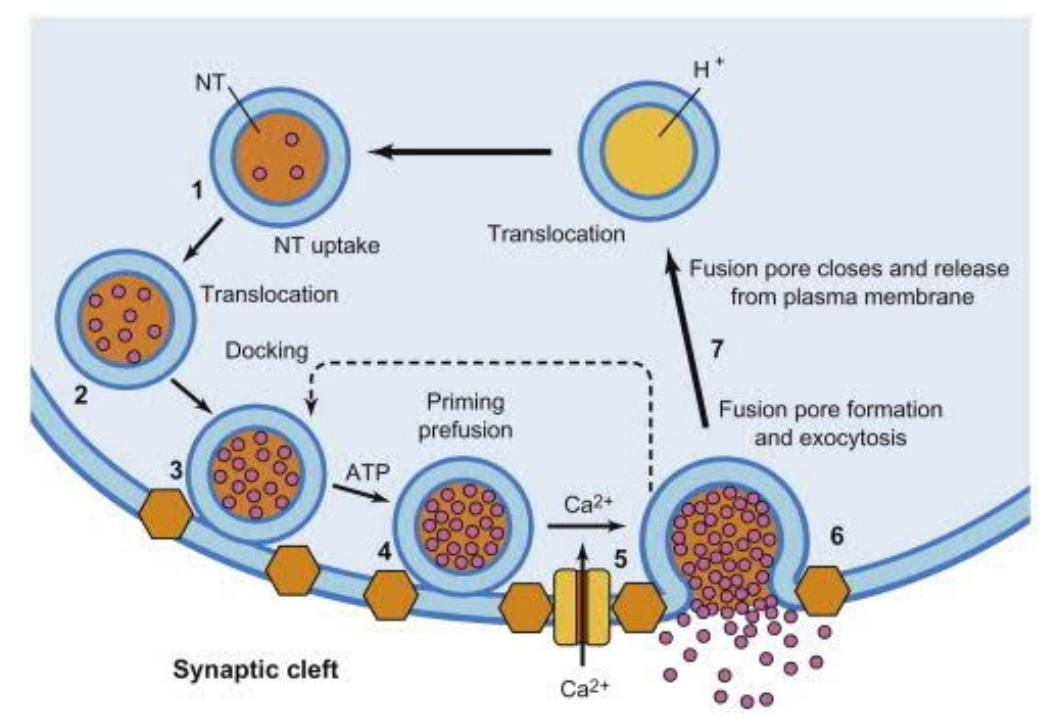

Figure 1: Synaptic vesicle cycle at the neuronal presynapse. 1: Synaptic vesicles are loaded with neurotransmitters. 2: Loaded vesicles are translocated near the active zone. 3: Docking of the vesicles near the active zone. 4: Priming of the vesicles at the active zone. 5: Influx of $\mathrm{Ca}^{2+}$ ions trigger the membrane fusion of vesicles with the plasma membrane at the active zone. 6: Membranes are recovered by endocytosis. 7: Vesicles are recycled and acidified. NT = neurotransmitter (16).

In order to restore synaptic vesicle pools, new vesicles are generated in a process called synaptic vesicle recycling. This might involve several strategies, however the process of clathrin-mediated endocytosis (CME) for vesicle recycling is the most established (23-27). In $\mathrm{CME}$, endocytosis is initiated by nucleation of the coat followed by the invagination of the pit, fission of the vesicle and finally the uncoating of the vesicle. 
After endocytosis and vesicle reformation, the synaptic vesicles are refilled with neurotransmitters. For neurotransmitter loading, the vacuolar $\mathrm{H}^{+}$-ATPase located on the vesicular membrane acidifies the vesicles (28-30). The high intravesicular concentration of protons establishes an electrochemical gradient that enables neurotransmitter transporters to load the vesicles (30-32). A recent, not yet peer-reviewed study determined the activity of the vacuolar $\mathrm{H}^{+}$-ATPase on filled synaptic vesicles as a mayor energetic burden that might account for almost $50 \%$ of the ATP consumed at the presynaptic bouton (33).

After acidification and neurotransmitter loading, the maturation of synaptic vesicles also requires the association for example with synapsin, rab3 or rabphilin before the vesicles relocate in the vesicle pool (34-36).

\section{Energy demands at the neuronal presynapse}

Neuronal signalling involves a variety of energy consuming processes. In total, synaptic transmission can make up to $55 \%$ of the total ATP expenditure of neuronal cells (37).

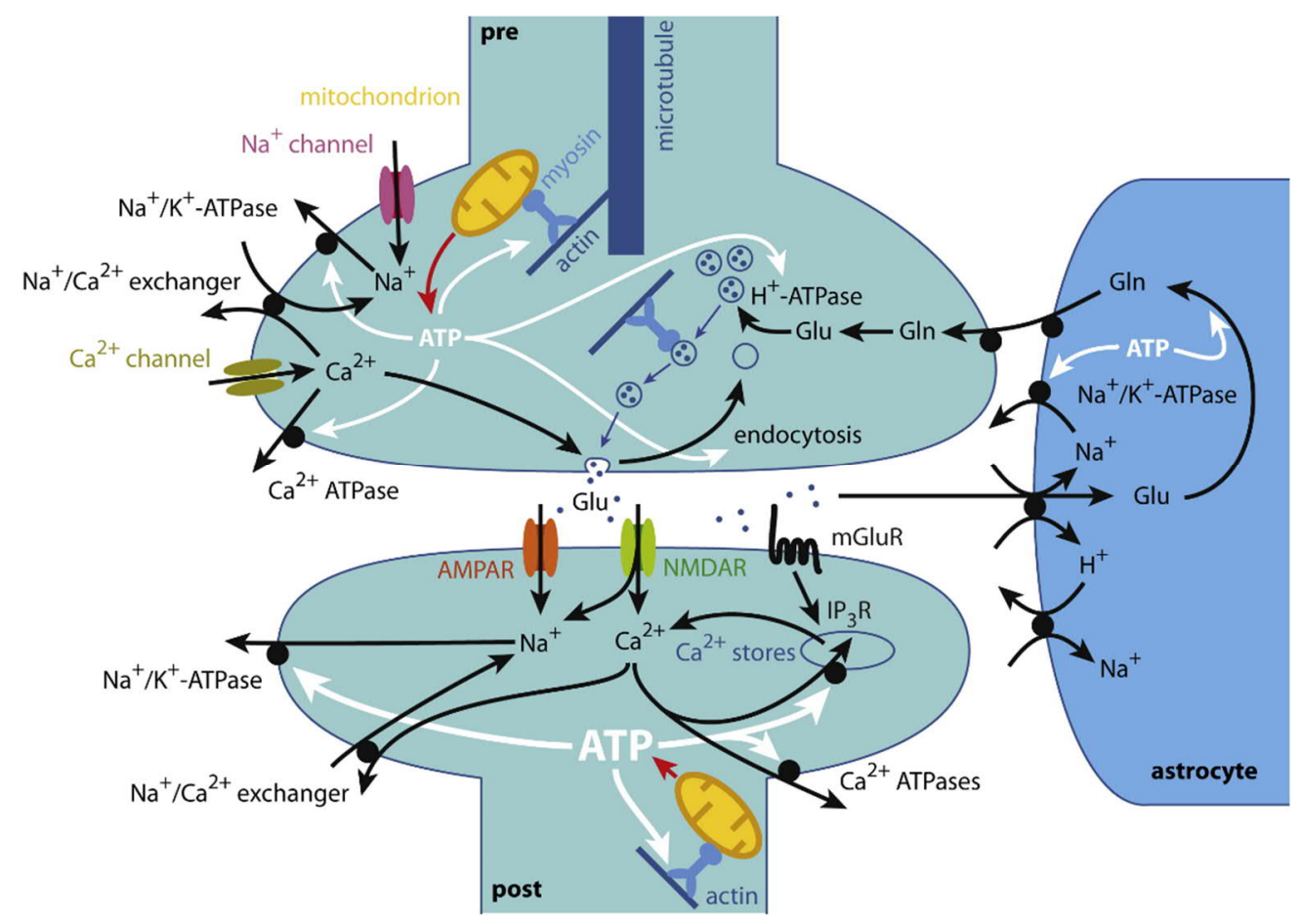

Figure 2: Energy consuming processes at the neuronal synapse. ATP consuming processes at the neuronal presynapse include the maintenance of ion levels such as $\mathrm{Na}^{+}, \mathrm{K}^{+}$and $\mathrm{Ca}^{2+}$. Further, ATP is required for the recycling of synaptic vesicles, the acidification and subsequent neurotransmitter loading of synaptic vesicles and finally the priming and release of synaptic vesicles (38).

The high local concentrations of glucose transporters in the presynaptic plasma membrane indicates the elevated energy demand in the presynapse. For example, a high extracellular concentration of glutamate provokes the surface accumulation of Glut3 transporters (39-41). 
Recent studies further suggest that Glut4 transporters are also crucial for glycolytic regulation at presynaptic sites (42-45).

The primary source of metabolic demand is the synaptic vesicle cycle (37). In addition, the active clearance of $\mathrm{Ca2}+$ ions to control synaptic strength as well as the re-establishment of sodium gradients for neuronal polarization requires energy (46). Furthermore, a sub fraction of presynaptic ATP might be involved in signalling (38) (Figure 2).

Because of the limited diffusion of ATP, these molecules have to be synthesised locally in the cells (47). For generation of energy derived from glucose breakdown at remote sites, neuronal cells can rely on two major strategies namely glycolysis or mitochondrial respiration (48). Glycolysis involves a series of enzymatic reactions in order to breakdown glucose. This process enables the extraction of energy for the cellular metabolism. The breakdown of glucose ultimately produces two molecules each of ATP, NADH and pyruvate as a product. This newly formed ATP fuels energy demanding processes (49-51).

In neuronal cell, strong evidence points towards glycolysis as the main energy supplier for sustained synaptic activity. First, the breakdown of glucose during glycolysis is thought to be relatively fast (51). Further studies demonstrated for example, that chemical inhibition of glycolytic enzymes by 2-deoxy-glucose caused a fast decline in local ATP levels at the presynapse. This further lead to a rapid decline in synaptic performance of the cells. In the same experiments, the inhibition of glycolysis blocked the endocytosis of synaptic vesicles suggesting that vesicle recycling as well is dependent on rapid ATP supply (52).

The dependency on glycolysis is also supported by recent findings showing the formation of large protein clusters consisting of glycolytic enzymes at sites of higher synaptic activity. Synaptosomes chemically fixed with aldehydes showed specific enrichment in five of ten glycolytic enzymes namely glucosephosphate isomerase, glyceraldehyde-phosphate dehydrogenase, aldolase, pyruvate kinase and lactate dehydrogenase (53). Enzymes like pyruvate kinase and lactate dehydrogenase mediate key energy producing steps in glycolysis. Further, the enzymes glyceraldehyde phosphate dehydrogenase (GAPDH) and 3phosphoglycerate kinase (3-PGK) next to others were found on synaptic vesicles $(54,55)$. This indicates that for example ATP derived from the two vesicle-bound enzymes supports vesicle acidification and neurotransmitter filling.

More recently, a study demonstrated a large cluster formed during energetic demands at presynaptic sites. The cluster that was termed glycolytic metabolon shows a local concentration increase of the enzymes phosphofructokinase, glyceraldehyde-3-phosphate dehydrogenase, pyruvate kinase and aldolase (56). The study pointed out a central role of glycolytic metabolon formation for the synaptic vesicle cycle. 
It is considered further, that glycolytic enzymes might be directly associated with the presynaptic $\mathrm{Na}^{+} / \mathrm{K}^{+}$pump that is necessary to restore neuronal polarization (57).

The aforementioned processes indicate a strong dependence of synaptic function on glycolysis and a failure of neuronal communication caused by insufficient glycolysis. However, in patients suffering from drug-resistance forms of epilepsy, a high ketogenic diet was shown as an effective treatment $(58,59)$. This suggest that neuronal presynapses can switch to a high activity of oxidative phosphorylation in situations where glycolysis is bypassed. How this change affects high-frequency activities of presynapses however is poorly understood to date. In a ketogenic metabolism, ATP at the presynapses would have to be replenished by oxidative phosphorylation that takes place in mitochondria (60). Mitochondrial contribution to ATP levels was recently shown to increase during elevated levels of $\mathrm{Ca}^{2+}$ in neuronal axons further indicating, that mitochondria might have a central role in support of synaptic function (60).

\section{Mitochondria - an overview}

Mitochondria form a dynamic network of organelles in almost every eukaryotic cell type. They are a key organelle for constant supply with ATP in the cells. Further, mitochondria participate for example in signalling of reactive oxygen species, clearance and storage of ions, immune responses and cell death $(61,62)$.
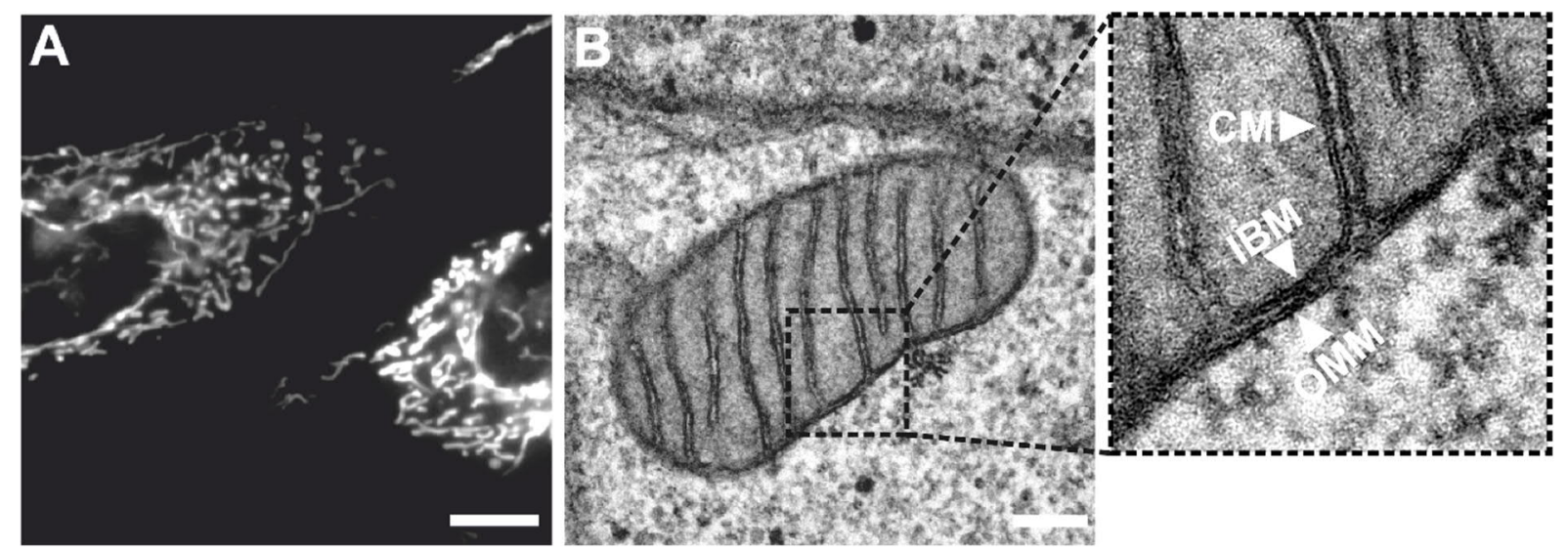

Figure 3: Mitochondria in HeLa cells. A: Live cell fluorescence microscopy of HeLa cells expressing a mitochondrial targeted mEosEM recorded with an epifluorescence microscope. B: Electron micrograph showing a mitochondrial cross section of a HeLa wildtype cell chemically immobilized and prepared for transmission electron microscopy. Boxed region in $B$ : Details of the mitochondrial membrane architecture. $C M=$ crista membrane. IBM = inner boundary membrane. OMM = outer mitochondrial membrane. Scale bar in $A=5 \mu \mathrm{m}$. Scale bar in $B=100 \mathrm{~nm}$. (Own data).

In light microscopy, fluorescently labelled mitochondria of cancer cells appear as a tubular network (Figure 3 A). In HeLa cells for example, the mitochondria range from a couple of hundreds nanometres up to a few micrometres in length and about 300 to $500 \mathrm{~nm}$ in diameter. 
Furthermore, mitochondria exhibit a complex membrane architecture that is traditionally investigated with electron microscopy $(63,64)$. This architecture consists of an outer mitochondrial membrane (OMM), the inner boundary membrane (IBM) in parallel to the outer membrane as well as the crista membranes (CM) (Figure $\mathbf{3} \mathbf{B}$, boxed region).

Anchor proteins located in the outer mitochondrial membrane facilitates the tethering and the transport of the organelle on cytoskeletal structures (65-68). The proper localization of mitochondria is an essential aspect to ensure mitochondrial support of cellular needs. The precise interaction of mitochondria with cytoskeletal components requires further investigations. However, for example the calcium-sensing GTPase Miro was identified as a central mediator of mitochondrial anchoring on microtubules. The interaction of Miro with motor proteins like dynein und kinesin for example allows travelling and repositioning of mitochondria in neuronal cells $(69,70)$.

Localized in the membrane are further important proteins for the in- and export of biological material, such as metabolites, lipids and proteins (71-75). Transporters localized in the outer membrane and the inner boundary membrane enable the passage of ions (76-79).

The membrane leaflets called crista membranes spanning across the mitochondrial matrix. The contact between the inner boundary membrane and the crista membranes is called crista junction. The crista junctions are maintained by the mitochondrial contact site and cristae organizing system (MICOS) $(64,80-83)$. The characterisation of the human MICOS and the involvement in crista membrane formation is in the centre of current investigations.

The crista membrane leaflets harbours the respiratory chain complexes and the rotary $F_{1} F_{0}$ ATP synthase (84). The respiratory chain complexes transport protons from the mitochondrial matrix into the intermembrane space between inner mitochondria membrane and outer mitochondrial membrane. The electrochemical gradient resulting from the proton shuffling enables the synthesis of ATP at the ATP synthase and allows for transport processes across the membranes (85).

The respiratory chain complexes I-IV together with ubiquinone, cytochrome-C and the ATP synthase form the electron transport chain (ETC). The ETC facilitates the generation of ATP in a process termed oxidative phosphorylation $(86,87)$. In oxidative phosphorylation, the oxidation of the energetic substrates $\mathrm{NADH}$ and $\mathrm{FADH}_{2}$ creates the energy to shuffle protons across the membrane. NADH is oxidized on complex I of the ETC and $\mathrm{FADH}_{2}$ on complex II respectively which ultimately drives the flow of $10 \mathrm{H}^{+}$across the membrane (88-91). The electrochemical gradient established by proton transfer drives the rotary $F_{1} F_{0}$ ATP synthase. The conformational change induced by the back flow of protons through $F_{0}$ allows then the 
phosphorylation of ADP to ATP (92). The energetic substrates NADH and FADH $\mathrm{F}_{2}$ that are needed in the ETC derive from a process called the tricarboxylic acid cycle (TCA).

In the mitochondria, pyruvate dehydrogenase converts pyruvate derived from glucose breakdown during glycolysis to acetyl-CoA (ACoA) (93). In the mitochondrial matrix, citrate synthase then condenses oxaloacetate with acetyl-CoA producing citrate that starts the TCA. The TCA generates the majority of $\mathrm{NADH}$ and FADH2 derived from carbohydrate oxidation that is required for the ETC. The full oxidation of glucose during glycolysis, the conversion of pyruvate to ACoA, and the TCA has a net yield of 30 molecules of ATP per molecule glucose. In comparison, glycolysis only generates two molecules of ATP. This clearly demonstrates that mitochondria utilize glucose more efficiently compared to glycolysis alone.

\section{Mitochondria in the neuronal synapse}

Neuronal cells account for a high carbohydrate turnover in any higher eukaryotes. In the adult human, the brain can consume up to $20 \%$ of the total calories despite its comparatively small size (94). Mitochondrial respiration provides the majority of the energy required for proper brain function (95).
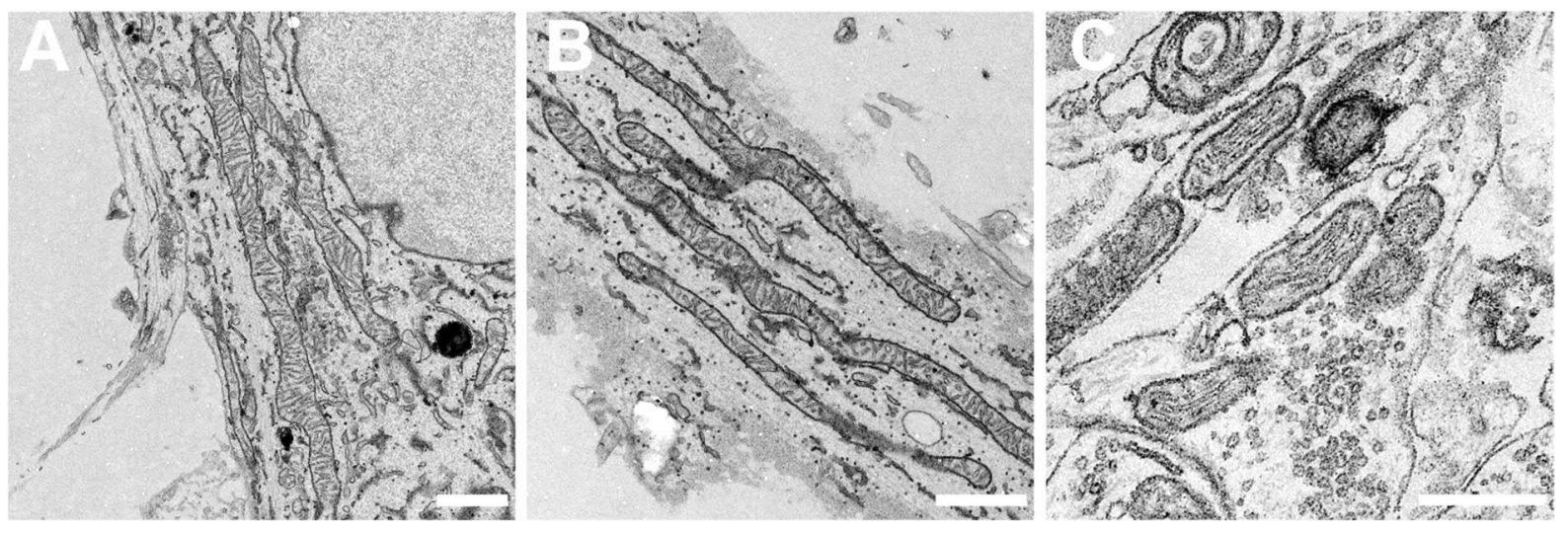

Figure 4: Transmission electron micrographs showing the mitochondrial structure in cultivated rat hippocampal neurons. A: Somatic mitochondria. B: Dendritic mitochondria. C: axonal and presynaptic mitochondria. Scale bar in $A=1 \mu \mathrm{m}$. Scale bar in $B=1 \mu \mathrm{m}$. Scale bar in $C=200$ nm. (Own data).

Depending on their positioning, neuronal mitochondria differ in size and appearance (Figure 4). Mitochondria in the neuronal soma and dendrites are large and elongated (Figure $4 \mathbf{A}$ and B), the axonal and presynaptic the individual mitochondria are usually small and puncta-like (96) (Figure $4 \mathrm{C}$ ). In all three compartments, the mitochondria are dynamic and the cells redistributes mitochondria on microtubule to cellular sites with higher energy demand (97).

The traveling of mitochondria to the axonal growth cone during development is crucial for the establishment of synaptic connections. Studies demonstrated a higher stability of presynapses 
occupied with mitochondria. This suggest that high levels of energy are required to form and maintain synaptic connections and that the demand can only be met by mitochondrial respiration (98).

As discussed previously, synaptic activity requires substantial energy. This would further argue for a high occupancy of presynapses with mitochondria. On the contrary, several studies in neurons of the central nervous system showed that only $20 \%$ to $40 \%$ of presynapses actually contain mitochondria (99-101). This might indicate a difference in functionality of the individual synapses with respect to frequency and strength of synaptic transmission.

Measurements using fluorescent reporter molecules for ATP revealed that indeed presynapses containing mitochondria showed elevated levels of ATP (102). The same studies pointed out, that presynaptic mitochondria might also contribute to ATP restoration in neighbouring boutons without mitochondria. Thus, a single mitochondrion could support fast recovery of ATP levels after synaptic activity in multiple boutons. This suggest that mitochondria could be located at distinctive points in the axon ensuring evenly distribution of energy. This is further supported by the observation that mitochondria might be anchored with cytoskeleton-like filaments to sites of high metabolic demand (103).

The initial observation of a low mitochondrial occupation of presynapses might be linked to an overall low activity of the neuronal cells. Thus, a long-term activation of synaptic transmission could lead to a re-localization of mitochondria to synapses. Light microscopy imaging revealed mitochondrial travelling to pre- and postsynaptic sites during stimulation in primary neurons $(104,105)$. However, additional studies demonstrated that the mitochondrial mobility declines in neuronal cultures during their maturation and ageing (100, 106, 107). Additionally, electron microscopic investigations of cultivated hippocampus slices stimulated for 120 minutes showed that the mitochondrial occupation of presynapses does not increase in the experiments (108). The electrical stimulation of the brain slices followed a theta burst pattern that is considered a long-term memory-inducing pattern of neuronal networks. However, the time scale of mitochondrial occupation might be much longer. Thus, further experiments are needed.

Mitochondrial stalling might lead to short-term association of mitochondria with active presynapses that does not result in synaptic occupation. In this process, $\mathrm{Ca}^{2+}$ ions derived from the presynaptic bouton bind to the Miro/TRACK complex of mitochondria. Mitochondria uncouple from their associated motor proteins such as Dynein and Kinesin and additionally tether via Syntaphilin to the microtubules $(109,110)$. Mitochondrial stalling can transiently supply ATP to meet energy demands at the presynapse. The exact contribution of mitochondrial stalling to presynaptic ATP pools is however not fully understood. 
Electron microscopy aided analyses demonstrated a structural adaptation of individual presynaptic mitochondria in-vivo. The calyx of Held $(\mathrm{CoH})$ is a large synapse in the auditory central nervous system. The $\mathrm{CoH}$ is a well-established model for a high performance presynapse and its structure was analysed in detail. Recently, major mitochondrial differences have been observed in mitochondria of the $\mathrm{CoH}$ during ageing of mice (111). Those differences included a general higher abundance of mitochondria at the terminal as well as a higher volume of the presynaptic mitochondria. This clearly indicates a long-term adaptation of the individual mitochondrial structure in high-performing synapses.

The $\mathrm{CoH}$ is a highly specialized presynaptic compartment. However, the majority of presynapses in the brain are comparatively small and much harder to identify and they might lack the extreme level of specialization. The structural adaptations in these smaller synapses is less well understood. In a recent approach, the ultrastructure of presynaptic mitochondria in GABAergic and glutamatergic boutons was investigated in mice and human (112). There, mitochondria in high-performing synapses (GABAergic) showed an increased size and volume as well as an increased lamellarity of the crista membranes compared to low-performing synapses (glutamatergic). This suggest a structural heterogeneity of presynaptic mitochondria in the brain.

Mitochondria show a specific membrane structure that is dependent on the cell type and the localization within the cell. In presynapses that are functionally different, they show different sizes and inner mitochondrial membrane architectures. The performance of these organelles is modulated by changes in the intracellular concentrations of $\mathrm{Ca}^{2+}$ ions (60). A high intracellular concentration of $\mathrm{Ca}^{2+}$ ions for example during synaptic activity leads to an increased activity of mitochondrial respiration. However, it is not understood how mitochondria adapt to an increased energy demand at the presynapse and specifically whether an increased activity of presynaptic mitochondria leads to a structural remodelling of the inner mitochondrial membranes. 


\section{Electron microscopy for cell biology}

Electron microscopy is a central tool to visualize and investigate cellular structures. In contrast to light microscopy, the specimen is investigated using electrons instead of light. Cellular details can be visualized at high details because electrons have a much smaller wavelength compared to light used in fluorescence microscopy. However, the interaction of electrons with cellular material causes local heating and emitting of $x$-rays due to inelastic scatter events of the electrons with the sample atoms. Furthermore, imaging with electrons requires highvacuum conditions in the microscope that will destroy hydrated samples. Therefore, the specimen has to be stabilized accordingly.

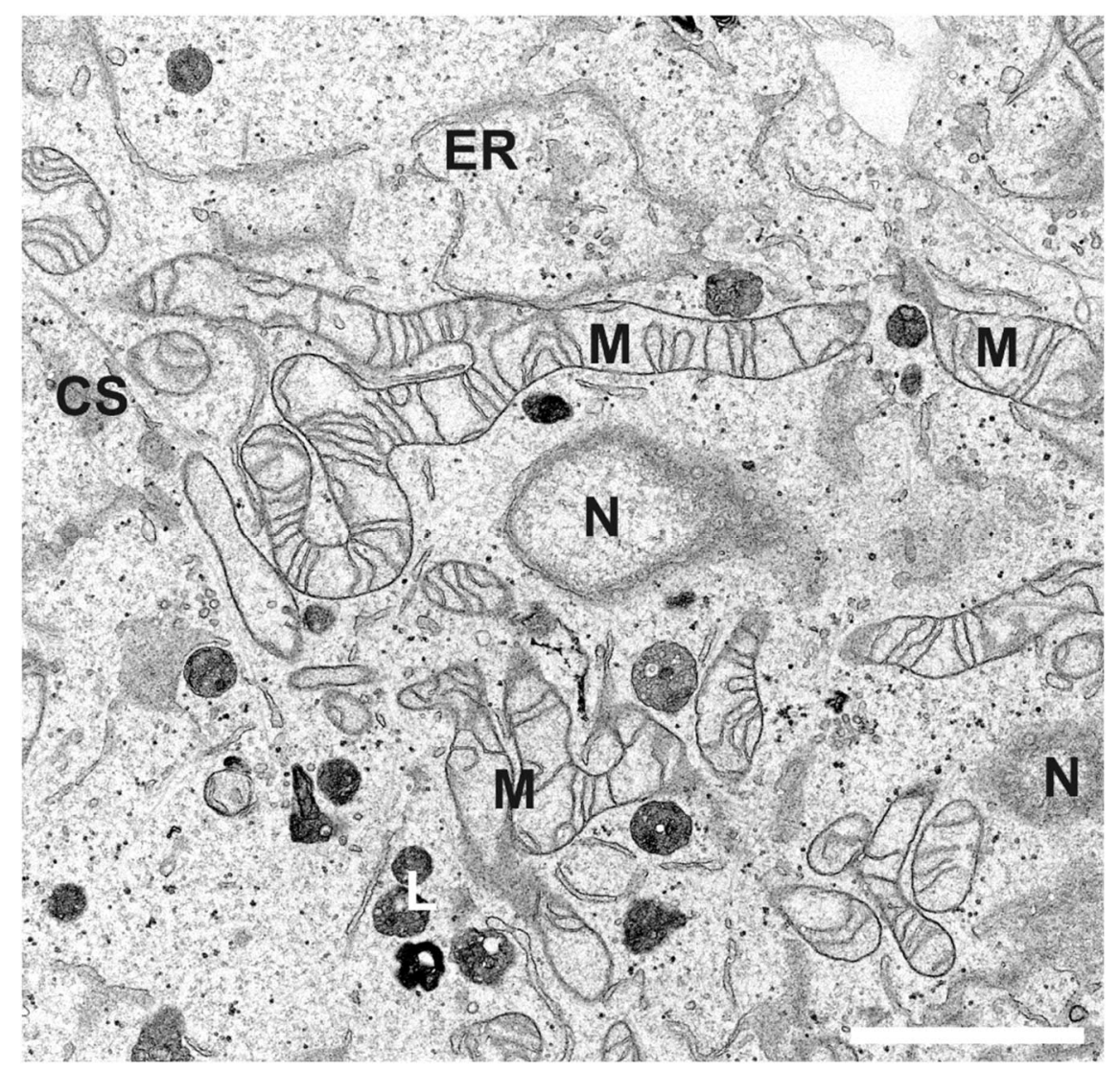

Figure 5: The ultrastructure of a HeLa wild type cell visualized by transmission electron microscopy. The electron micrograph shows multiple mitochondria, sheets of the endoplasmic reticulum, lysosomal vesicles, multiple other small vesicles and cytoskeleton, peripheries of the nucleus including the nuclear envelope and nuclear pores. $M=$ mitochondria. $E R=$ endoplasmic reticulum. $N=$ nucleus. $L=$ endosomal vesicles/lysosomes. $C S=$ cytoskeleton . Images were recorded at $120 \mathrm{kV}$ with $4500 x$ original magnification. Scale bar $=2 \mu \mathrm{m}$. (Own data).

In classical preparations of biological material, proteins are first immobilized by aldehydemediated crosslinking. Subsequently the samples are stained with heavy metals such as osmium tetroxide and uranyl acetate. Osmium tetroxide stabilizes cell membranes due to crosslinking of unsaturated lipids (113). Uranyl acetate forms insoluble precipitates with 
organophosphates thus, staining proteins within the cell as well as similar functional groups on membrane lipids $(114,115)$. Finally, the samples are then dehydrated and embedded in a resin. This allows cutting (ultra) thin sections of the specimen that can be viewed in the microscope. Biological structures are fragile and inherently low in contrast when imaged in electron microscopy. Staining and resin embedding helps to stabilize the cellular structures for imaging in high vacuum with an electron beam as well as increasing their contrast (116).

Due to the nature of the staining reagents used, all structures are stained mostly unspecific. This holds the advantage that the reference space of all organelles and structures is visible providing valuable context information (Figure 5).

The sample preparation for traditional electron microscopy can introduce significant artefacts within the sample. First, due to the slow immobilization by aldehyde crosslinking, structures can move for extended time before they are fixed in place (117). In addition, chemical immobilization can cause sample perturbations due to changes in osmolarity as well as the $\mathrm{pH}$ value. Next, the staining with heavy metals creates a cast around biomolecules. Thus, the electron micrographs show a delineation of structures by heavy metals and not the structures themselves. Finally, sample dehydration and resin embedding significantly changes the environment of the biomolecules (118).

All of the associated artefacts have to be considered for interpretation of the data. A major breakthrough addressing these limitations was the development of cryo-immobilization and electron microscopy of cells at cryogenic temperatures (119-123). Here, the samples are stabilized in a layer of amorphous ice. This can be obtained for example by high-pressure freezing of the material $(124,125)$. A pressure of about 2100 bar as well as fast cooling rates are applied to the sample. This prevents the formation of large ice crystals that otherwise would disturb biological structures. The more common approach for cell monolayers however, is plunge freezing of the cells directly in liquid ethane (126). Biological structures in aqueous milieu are immobilized within milliseconds. Thus, cryo electron microscopy allows in-situ investigations of cell membranes as well as proteins. Despite all the advantages, cryo electron microscopy suffers from a very low throughput as well as time-consuming preparation steps. Traditional room temperature is comparatively straight forward and many cells can be analysed in a short time. Thus, for most applications in cell biology, room temperature electron microscopy plays a significant role.

Current applications of electron microscopy involve high-resolution transmission electron microscopy in 2D as well as electron tomography to study the delicate structural arrangements within the neuronal presynapse $(127,128)$. There, the precise architecture of the active zone and synaptic vesicle pools was deciphered. Important findings include measurements of 
docked vesicles under certain conditions. It is considered, that these values could be used to estimate synaptic performance.

Furthermore, a filament like organisation machinery of synaptic vesicles was described recently $(129,130)$. Other findings described the unique aspects of mitochondrial distribution within the axon (131) or even the shuttling of organelles between neuronal cells via tunnelling nanotubes $(132,133)$. These processes were captured in high detail with a few nanometres resolution.

Transmission electron microscopy is conducted on thin resin sections. Thus, the sample volume that can be investigated is limited to a few hundred nanometres. Strategies to image large sample volumes involves scanning electron microscopy (SEM). Slice and view techniques like serial blockface or focused ion beam milling combined with SEM are used extensively to visualize the complex connections formed by several neuronal cells and their cellular arrangements within the brain (134-136). Volume-SEM applications benefit from a comparatively large field of view was well as the capability to image thick samples layer by layer (137).

As a major analytical method in life science, electron microscopy is a central tool in strengthening our understanding of neuronal cell biology and neuronal mitochondria in particular.

\section{Correlative Imaging of cellular structures}

Electron microscopy is limited in specific labelling strategies of cellular components and proteins in order to understand functional aspects. Light microscopy on the other side can highlight the position of a specifically labelled biomolecule with high precision. However, light microscopy can only provide a limited understanding of structural details within a cell. A correlation of light and electron microscopy can alleviate those limitations.

In the first correlative light and electron microscopy (CLEM) experiments, cells were first identified in bright field light microscopy. Flat fibroblast cells of homogenized chicken embryos only immobilized with osmium tetroxide were identified and these cells subsequently transferred to the electron microscope. This allowed for the first time to visualize cellular structures in electron microscopy (138).

For advanced CLEM imaging, a breakthrough was achieved with the discovery and implementation of fluorescent proteins in cell biology (139). This allows visualizing specific proteins and gives rise to functional information in both, fixed cells and in living cells. To date varieties of fluorescent reporters are accessible. Those include fluorescent proteins, potential 
driven chemical dyes and self-labelling enzymes in conjunction with inorganic dyes $(140,141)$. To date, multiple sample preparation strategies for correlative imaging are available (Figure 6).

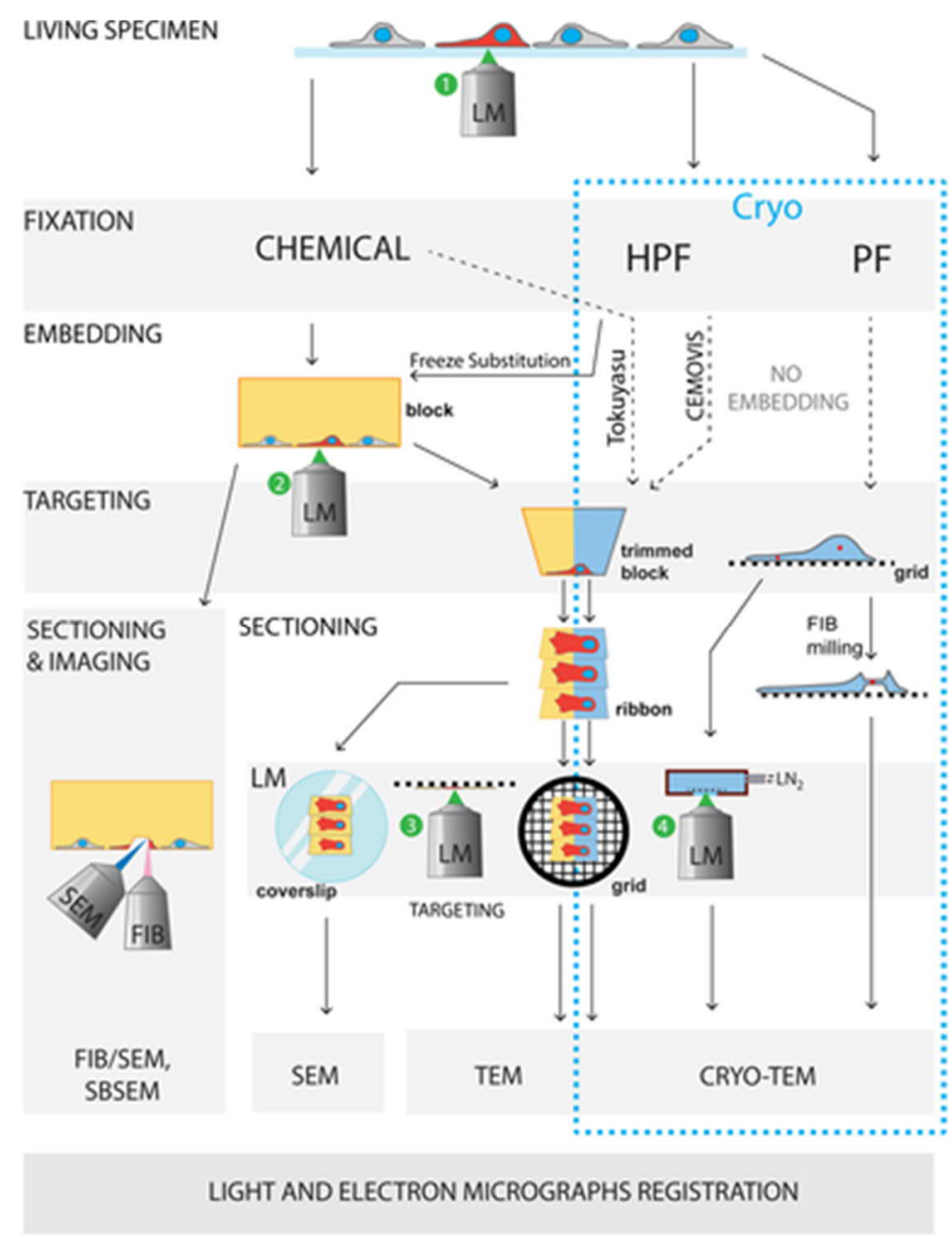

Figure 6: Sample preparation routes of biological specimens for correlative light and electron microscopy. The chart displays possible strategies to combine information obtained by light and electron microscopy of one sample. This ranges from room temperature pre-embedding CLEM approaches to cryo light and cryo electron microscopy. $L M=$ light microscopy. HPF = high-pressure freezing. $P F=$ plunge freezing. $F I B=$ focussed ion beam. $S E M=$ scanning electron microscopy. SBSEM = serial blockface SEM. TEM = transmission electron microscopy (142).

A combination of light microscopy imaging with subsequent room temperature embedding and analysis using electron microscopy is called pre-embedding CLEM (Figure 7). In preembedding CLEM, both imaging modalities are performed separately, which allows operating both, light microscopy as well as electron microscopy at high performance parameters. A powerful aspect of this approach is the correlation of live cell processes with structural information. 


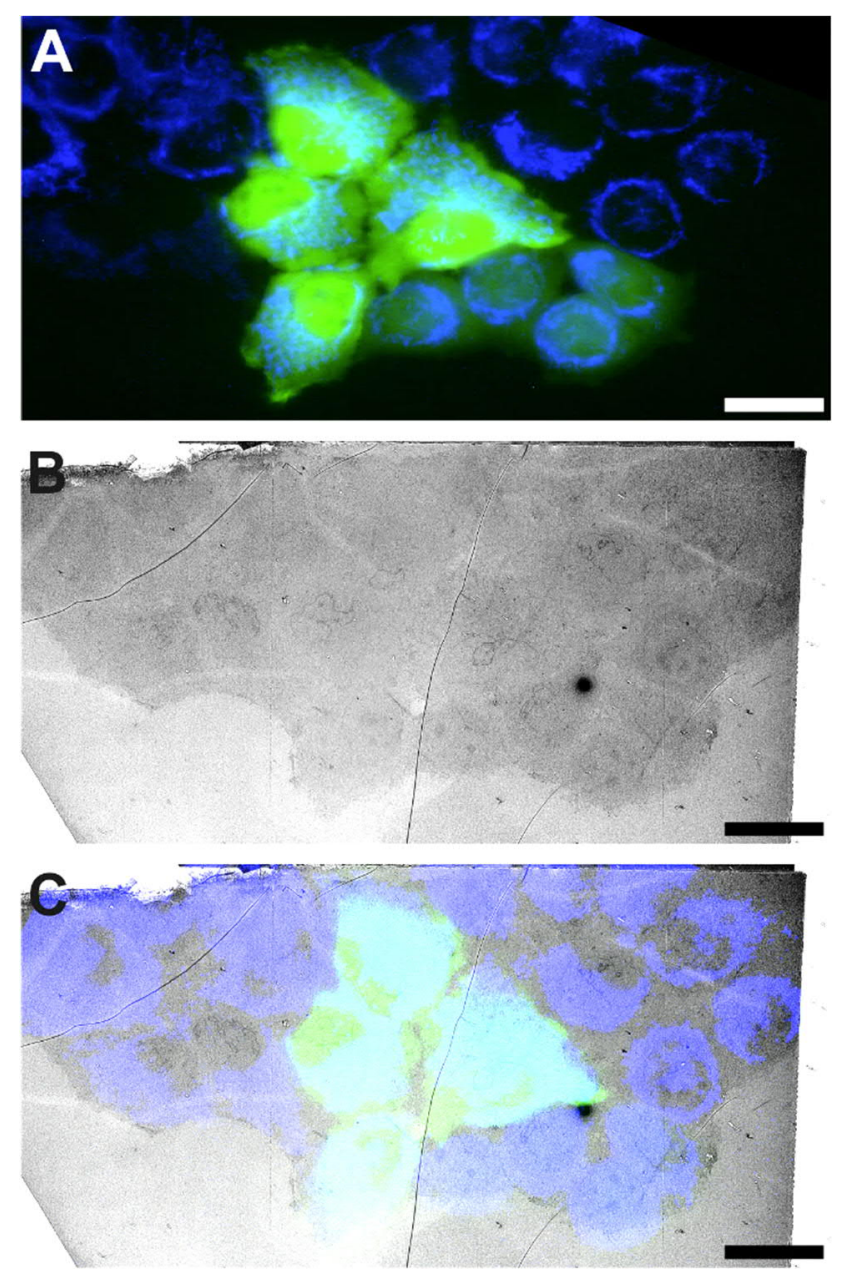

Figure 7: Live cell CLEM enables identification of targets. A: Live cell imaging of HeLa cells stained with Mitotracker Deep Red FM (blue) and a cytoplasmic EGFP reporter (green). B: Transmission electron micrograph of the same cell population. C: Correlation and overlay of the light microscopy images on the electron micrograph provides the location of targetexpressing cells for subsequent detailed investigations. Scale bars $=20 \mu \mathrm{m}$. (Own data).

Most strategies rely on the immobilization by means of aldehyde mediated chemical crosslinking. As discussed earlier, a major limitation of this approach is the possible introduction of significant artefacts in the sample during staining and embedding for electron microscopy. This might limit the precision of registration of the two data sets.

Furthermore, retrieval of the area of interest first imaged in light microscopy can become challenging especially for three-dimensional correlations. Some of these problems can be addressed in an approach called post-embedding CLEM (143).

In post-embedding CLEM, all imaging is carried out in a specimen already immobilized and prepared for light and electron microscopy. With the development of high-pressure freezing (HPF) for the immobilization of biological specimens, protocols for post-embedding CLEM advanced. During subsequent freeze substitution and embedding in methacrylate resins, the fluorescence of proteins and inorganic dyes can be preserved. Recently an automated imaging 
platform with coupled high-pressure freezing was introduced that would allow a minimal time delay between fluorescence microscopy and the immobilization of the cells (144). This system would allow combining live cell imaging with post-embedding CLEM.

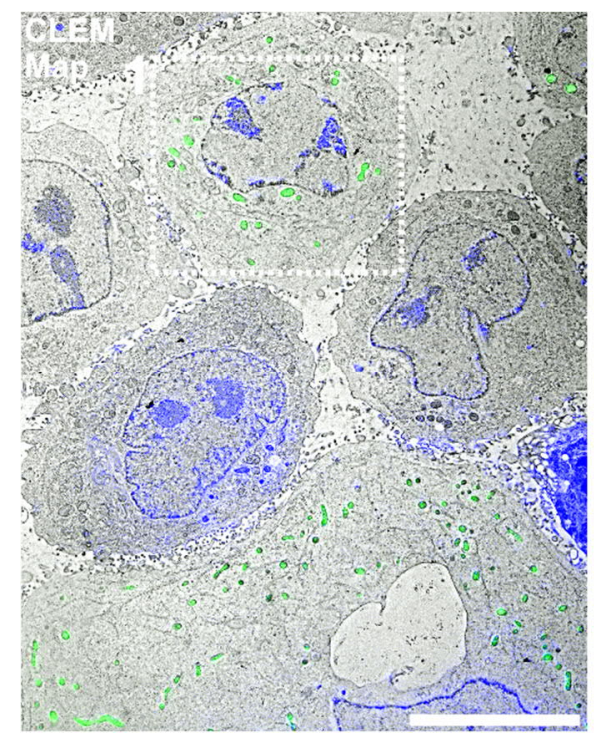

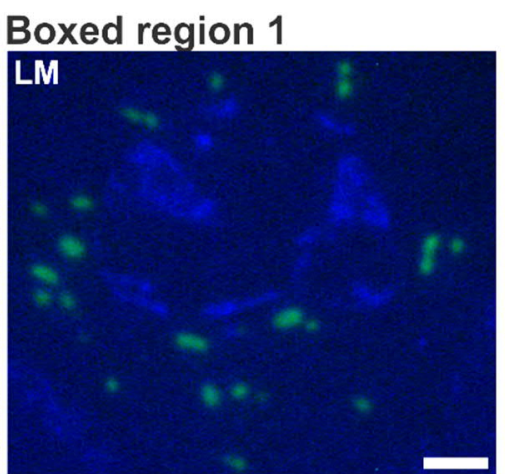

Boxed region 2

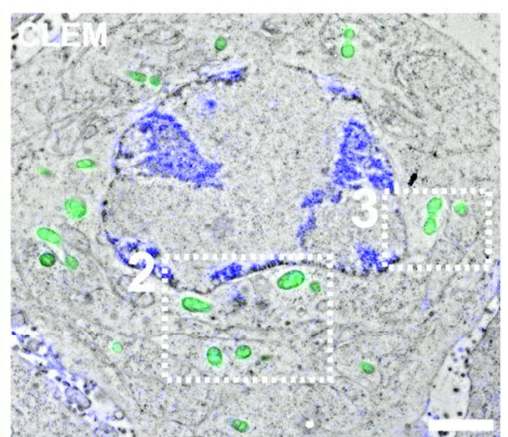

Boxed region 3

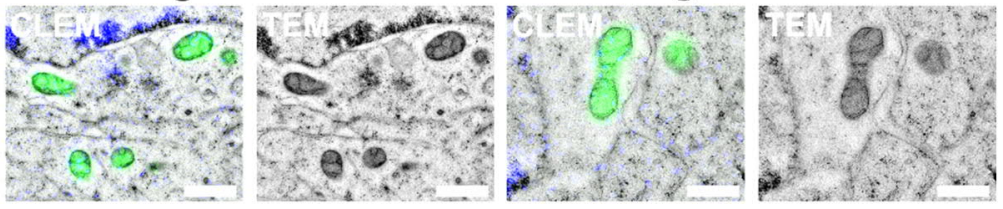

Figure 8: Correlative light and electron microscopy of HeLa cells expressing a mitochondria targeted $m$ Citrine. A registration of overview images obtained by light and electron microscopy results in a CLEM-map highlighting areas of interest for subsequent detailed analysis. Subareas within the sample are then subjected to high magnification imaging in electron microscopy revealing the position of the protein of interest within its structural environment. Scale bar in CLEM-Map $=10 \mu \mathrm{m}$. Scale bar in boxed region $1=1 \mu \mathrm{m}$. Scale bars in boxed region 2 and $3=500 \mathrm{~nm}$ (145).

For post-embedding CLEM, the fluorophores are imaged in the thin sections prepared for electron microscopy (Figure 8). Due to the minimalized error in registration, this technique is also referred to as high-accuracy CLEM. This approach was used to determine the position of various membrane bound proteins in their underlying structure with high spatial precision. In combination with multicolour labelling of proteins this even allows to dissect time dependent interactions of the proteins at the membranes $(146,147)$.

Correlative imaging can increase the throughput of electron microscopy investigations in multiple ways. As discussed earlier, cells of interest are identified in light microscopy. This guides imaging to subcellular areas that might not be recognisable in electron microscopy alone. Secondly, it was recently demonstrated that mixed populations of cells could be discerned via fluorescence microscopy thus allowing for specific screening of one population within the sample (148).

Correlative imaging could be applied for neuronal imaging as well. First studies applied CLEM in cultivated hippocampal neurons (149-151). These studies further suggest that live cell CLEM 
could be applied to correlate neuronal activity to presynaptic architecture and presynaptic mitochondria in particular.

\section{Aims of the thesis:}

Synaptic transmission poses a major energy burden of the brain. In the brain, mitochondria are key organelles that provide energy needed for the survival of neuronal cells. The mitochondrial plasticity reflects their functionality and adaptations in different cell types and to changing conditions. However, to date the structural adaptation of presynaptic mitochondria to changes in neuronal activity are poorly understood.

Thus, the goal was of this thesis was to gain detailed insights into the structural adaptation of mitochondria in synapses to varying energy demands. These findings will provide essential information that will ultimately help to understand the exact role of mitochondria in synaptic transmission.

Initially, cultured cancer cells will be used as a model system to study the changes of mitochondrial structure as a response to different energy sources. Subsequently, mitochondria in the presynapses of rat hippocampal neurons will be analysed with respect to their structure and abundance during resting periods and stimulated activity of the neuronal cells. These findings will then be compared to in-vivo data on presynaptic mitochondria in mice at different stages of development.

For the visualization of the mitochondrial ultrastructure in different cells and subcellular compartments transmission electron microscopy (TEM) will be used as a method. To this end, protocols for the fixation, staining and visualization of the cellular ultrastructure with a focus on the mitochondrial ultrastructure in different mammalian cells and tissues for transmission electron microscopy will be established and compared.

Finally, in order to gain additional mechanistic insights to mitochondrial function, tools for correlative imaging will be developed which will allow combining functional information with ultrastructural data. Initially, protocols for pre- and post-embedding correlative light and electron microscopy (CLEM) will be established on HeLa cells and then combined with metabolic imaging. This correlative approach will allow extracting information about protein localization, cellular structure and chemical composition on a sub cellular level in HeLa cells. 


\section{Methods}

\section{Cell culture}

HeLa, Cos7 and U-2 OS cells were cultivated in DMEM high glucose medium (Thermo Fischer Scientific) further supplemented with 1x GlutaMAX additive (4 mM final, Thermo Fisher Scientific), $100 \mathrm{U} / \mathrm{ml}$ penicillin and $100 \mu \mathrm{g} / \mathrm{ml}$ streptomycin (Merck Millipore), $1 \mathrm{mM}$ sodiumpyruvate (Sigma Aldrich) and $10 \%(\mathrm{v} / \mathrm{v})$ fetal bovine serum (Merck Millipore) in an incubator set to $37^{\circ} \mathrm{C}$ with $5 \% \mathrm{CO}_{2}$.

\section{Special cell culture medium}

\section{Low glucose culture medium}

Culture medium containing low glucose consisted of SILAC FLEX medium (Thermo Fisher Scientific) supplemented with $10 \%$ (v/v) dialyzed fetal calf serum (Thermo Fisher Scientific), $100 \mathrm{U} / \mathrm{ml}$ penicillin and $100 \mathrm{\mu g} / \mathrm{ml}$ streptomycin and $5 \mathrm{mM}$ glucose (Sigma Aldrich).

\section{Ketone bodies culture medium}

Culture medium containing ketone bodies consisted of SILAC FLEX medium supplemented with $10 \%(\mathrm{v} / \mathrm{v})$ dialyzed fetal calf serum, $100 \mathrm{U} / \mathrm{ml}$ penicillin and $100 \mu \mathrm{g} / \mathrm{ml}$ streptomycin and $25 \mathrm{mM}$ beta-hydroxybutyrate (Thermo Fisher Scientific).

\section{Pyruvate culture medium}

Culture medium containing sodium-pyruvate consisted of SILAC FLEX medium supplemented with $10 \%(\mathrm{v} / \mathrm{v})$ dialyzed fetal calf serum, $100 \mathrm{U} / \mathrm{ml}$ penicillin and $100 \mu \mathrm{g} / \mathrm{ml}$ streptomycin and $10 \mathrm{mM}$ sodium-pyruvate.

\section{Cultivation of rat hippocampal neurons}

\section{Hippocampus isolation and dissociation}

Rat hippocampi were obtained by resection of the brain of Wistar-rats. Newborn rats (P0 or P1) were decapitated with a large scissor and the skin removed with a pair of forceps. The skull was opened with a small scissor only making shallow cuts in order to preserve the undamaged brain. The brain was removed with a spatula and placed in a $3.5 \mathrm{~cm}$ culture dish filled with ice cold HBSS no calcium, no magnesium (Thermo Fisher Scientific). The two 
hemispheres were separated with a sharp spatula and the meninges removed using two pairs of forceps. The hippocampus was isolated by cutting with a small scissor and a pair of hippocampi collected in a $15 \mathrm{ml}$ Falcon-tube and stored on ice. For the dissociation the hippocampi were placed in $4.5 \mathrm{ml}$ ice cold HBSS no calcium, no magnesium and $0.5 \mathrm{ml}$ of a freshly thawed $2.5 \%$ trypsin (Thermo Fisher Scientific) added to the hippocampi. Dissociation took place for $18 \mathrm{~min}$ in a water bath set to $37^{\circ} \mathrm{C}$ with occasional light shaking of the tubes. Digestion was stopped by adding $10 \mathrm{ml}$ of pre-warmed DMEM supplemented with $5 \%$ FBS to each Falcon-tube and mixing. The tissues were then centrifuged at $100 \mathrm{~g}$ for $3 \mathrm{~min}$. The supernatant was removed and the tissues washed twice with $10 \mathrm{ml}$ pre-warmed HBSS no calcium, no magnesium each. The tissues were then dissociated with a $5 \mathrm{ml}$ plastic Pasteur pipette by pipetting up and down the tissue pieces in approximately $5 \mathrm{ml}$ Neurobasal plating medium. The cell-suspension was then placed on a $40 \mu \mathrm{m}$ cell strainer and collected in a 50 $\mathrm{ml}$ Falcon-tube. The final cell-suspension was achieved by diluting the strained cell-suspension with pre-warmed Neurobasal plating medium to achieve a final cell count of $1 \cdot 10^{6} \mathrm{cells} / \mathrm{ml}$. Cells were then plated by adding $1 \mathrm{ml}$ of the final cell-suspension onto $18 \mathrm{~mm}$ glass coverslips. One hour after plating the supernatant was exchanged for standard cultivation medium.

\section{Neuronal cell media}

\section{Neurobasal plating medium}

Standard medium for the cultivation of rat hippocampal neurons consisted of Neurobasal-A medium supplemented with $0.5 \mathrm{mM}$ GlutaMAX, $0.5 \mu \mathrm{M}$ glutamate and $1 \times \mathrm{B}-27$ supplement. All media were sterile-filtered $(0.2 \mu \mathrm{m}$ filter) prior to use and conditioned by warming up in the incubator.

\section{Standard cultivation medium}

Standard medium for the cultivation of rat hippocampal neurons consisted of Neurobasal-A medium supplemented with $0.5 \mathrm{mM}$ GlutaMAX, PenStrep, 1x CultureONE supplement and 1x B-27 supplement (all Thermo Fisher Scientific). All media were sterile-filtered prior to use and conditioned by warming up in the incubator.

\section{Pyruvate neuronal refeed medium}

Neuronal culture medium containing pyruvate consisted of Neurobasal-A medium without glucose and pyruvate supplemented with $10 \mathrm{mM}$ sodium-pyruvate, PenStrep, CultureONE supplement and B-27 supplement (all Thermo Fisher Scientific). 


\section{Ketone bodies neuronal refeed medium}

Neuronal culture medium containing pyruvate consisted of Neurobasal-A medium without glucose and pyruvate supplemented with $25 \mathrm{mM}$ beta-hydroxybutyrate, PenStrep, CultureONE supplement and B-27 supplement (all Thermo Fisher Scientific).

\section{Buffer solutions}

\section{0x Phosphate buffered saline (PBS) stock}

Stock solutions of 10x PBS buffer were obtained by mixing and dissolving $1.37 \mathrm{M} \mathrm{NaCl}, 27$ $\mathrm{mM} \mathrm{KCl}, 100 \mathrm{mM} \mathrm{Na}_{2} \mathrm{HPO}_{4}$ and $18 \mathrm{mM} \mathrm{KH}_{2} \mathrm{PO}_{4}$ in vapour-distilled water.

\section{Phosphate buffered saline working solutions}

Working solutions of PBS were obtained by diluting the 10x PBS stock with vapour-distilled water until the desired final concentration was achieved. The $\mathrm{pH}$ was adjusted by either using $0.1 \mathrm{M} \mathrm{HCl}$ or $0.1 \mathrm{M} \mathrm{NaOH}$ solutions.

\subsection{M cacodylic buffer pH 7.4}

Final working solutions of $0.1 \mathrm{M}$ cacodylic buffer were obtained by mixing $0.2 \mathrm{M}$ cacodylic buffer (EMS, pH 7.4) with an equal volume of double distilled $\mathrm{H}_{2} \mathrm{O}$. The $\mathrm{pH}$ was checked again after mixing.

\subsection{Sorensen phosphate buffer}

Stock solutions of Sorensen phosphate buffer (SPB) were obtained by mixing a $0.2 \mathrm{M}$ $\mathrm{Na}_{2} \mathrm{HPO}_{4}$ solution with a volume of $0.2 \mathrm{M} \mathrm{KH}_{2} \mathrm{PO}_{4}$ solution until the desired $\mathrm{pH}$ of 7.4 was achieved. Stock solutions were stored in the dark at $4{ }^{\circ} \mathrm{C}$ and discarded as soon as microbial growth became obvious.

\subsection{Sorensen phosphate buffer}

Final working solutions were obtained by mixing a stock solution of $0.2 \mathrm{M}$ Sorensen phosphate buffer with an equal amount of double distilled water. The $\mathrm{pH}$ was checked again after mixing. Final solutions were stored in the dark and discarded as soon as microbial growth became obvious. 


\section{Sample preparations of cells for transmission electron microscopy (TEM) Preparation of CLEM finder substrates}

Finder-grids of choice were placed on top of either Aclar polymer film or the top part of high pressure freezing metal planchettes (Wohlwend). A negative replica of the TEM finder grid was obtained by sputter dispersion of evaporated carbon with a thickness of 8 to $12 \mathrm{~nm}$. The sample substrates were then treated with $70 \%$ ethanol to sterilize prior to use.

\section{Cell immobilization for chemical aldehyde fixation (CAF)}

Cells were immobilized using a freshly prepared and pre-warmed solution of $2.5 \%$ glutaraldehyde (Science Services) in $0.1 \mathrm{M}$ cacodylic buffer at pH 7.4 if not stated otherwise. Approximately $80 \%$ of the cell culture medium was aspirated and $2 \mathrm{ml}$ of the freshly prepared fixative added to the cells. Fixative was replaced with fresh fixative after 15 minutes and the immobilization was continued for 45 minutes at room temperature and subsequently for an additional 18 hours at $4^{\circ} \mathrm{C}$.

\section{Standard staining procedure for electron microscopy}

Prior to the secondary fixation, the samples were washed 4 times with $0.1 \mathrm{M}$ cacodylic buffer at $\mathrm{pH}$ 7.4. The first two buffer exchanges were immediate and the last two exchanges for 5 minutes each. Secondary fixation was facilitated using $1 \%$ osmium tetroxide (Science Services) in $0.1 \mathrm{M}$ cacodylic buffer at $\mathrm{pH} 7.4$ for one hour at room temperature. Afterwards, the samples were washed 4 times with double distilled water with the first two exchanges immediate and the following two exchanges for 5 minutes each. Samples were en-bloc stained with $1 \%$ uranyl acetate (EMS) in double distilled water for 30 minutes at room temperature in the dark. Dehydration followed an increasing ethanol gradient starting with $30 \%$ over 50,70 up to $100 \%$ with several exchanges in-between. Final dehydration prior to resin infiltration was facilitated with two exchanges of propylene oxide (ReagentPlus $\geq 99 \%$, Sigma Aldrich) for 5 minutes each. Resin infiltration was mediated with a starting solution of 1:1 Epon resin (Embed 812 kit, Science Services) and propylene oxide for one hour at room temperature followed by placing the samples into fresh $100 \%$ Epon resin for an additional hour. For overnight infiltration, the samples were placed again in fresh Epon resin on a rocker table over night at room temperature. The following day the samples were embedded in BEEM capsules (Science Services) and resin polymerisation took place over 48 hours at $60^{\circ} \mathrm{C}$. 


\section{High membrane contrast procedure for electron microscopy}

Sample preparation was changed to the "high membrane contrast" protocol with the following aspects. Cacodylic buffer was completely replaced with $0.1 \mathrm{M}$ Sorensen's phosphate buffer at $\mathrm{pH}$ 7.4. Secondary fixation was started using $1 \%$ osmium tetroxide in $0.1 \mathrm{M}$ Sorensen's phosphate buffer for one hour at room temperature. Without any washing in-between the solution got replaced with $2 \%$ osmium tetroxide in $1.5 \% \mathrm{~K}_{4}\left[\mathrm{Fe}(\mathrm{CN})_{6}\right]$ in $0.1 \mathrm{M}$ Sorensen's phosphate buffer and incubated for an additional 40 minutes at room temperature. Samples were washed first for three times with double distilled water and two additional times with 0.05 $\mathrm{M}$ maleate buffer at $\mathrm{pH} 5.51$. For the conditioning of the samples, the last incubation step with maleate buffer was carried out for 5 to 10 minutes. Samples were then en-bloc stained with 1 $\%$ uranyl acetate and $2 \%$ samarium trichloride (Sigma Aldrich) in $0.05 \mathrm{M}$ maleate buffer at $\mathrm{pH}$ 5.51. After three washing steps with double distilled water for 5 minutes each, the samples were dehydrated with a graded ethanol series starting with $30 \%$ over 50,70 up to $100 \%$ with several exchanges in-between. Final dehydration prior to resin infiltration was facilitated with two exchanges of propylene oxide for 5 minutes each. Resin infiltration was mediated with a starting solution of 1:1 Epon resin and propylene oxide for one hour at room temperature followed by placing the samples into fresh $100 \%$ Epon resin for an additional hour. For overnight infiltration, the samples were placed again in fresh Epon resin on a rocker table over night at room temperature. The following day the samples were embedded in BEEM capsules and resin polymerisation took place over 48 hours at $60^{\circ} \mathrm{C}$.

\section{fBROPA protocol for serial blockface SEM imaging}

This protocol was largely adapted from a recent publication with changes made to the timing of individual steps. Cells were immobilized using a pre-warmed solution consisting of $2.5 \%$ glutaraldehyde in $0.1 \mathrm{M}$ Sorensen phosphate buffer at $\mathrm{pH} 7.4$. Fixation was continued for one hour at room temperature and was completed over night at $4{ }^{\circ} \mathrm{C}$. In case the supernatant turned yellowish in the process, the fixative was replaced with fresh solution. Secondary fixation was started using $1 \%$ osmium tetroxide in $1.5 \% \mathrm{~K}_{4}\left[\mathrm{Fe}(\mathrm{CN})_{6}\right]$ in $0.1 \mathrm{M}$ Sorensen's phosphate buffer for one hour at room temperature. Without any washing in-between, the solution was replaced with $1 \%$ osmium tetroxide in $0.1 \mathrm{M}$ Sorensen's phosphate buffer and incubated for an additional hour at room temperature. Followed by three washing steps with double distilled water for 5 minutes each, the samples were incubated in a solution of $320 \mathrm{mM}$ pyrogallol un-buffered in double distilled water ( $\mathrm{pH}$ at about 4.1) for 30 minutes. The samples were then washed three times with double distilled water for 5 minutes each and incubated in a solution of $1 \%$ osmium tetroxide un-buffered in double distilled water. During three extensive washing steps using double distilled water for 10 minutes each, the Walton's lead aspartate 
solution was prepared. Therefore, $40 \mathrm{mg}$ aspartic acid were dissolved in double distilled water and the solution brought up to $60{ }^{\circ} \mathrm{C}$ in an oven. Next, $66 \mathrm{mg}$ lead nitrate were added to the solution. The $\mathrm{pH}$ of the mixture was then adjusted using $1 \mathrm{~N}$ sodium hydroxide solution. Care has to be taken that the mixture remained at $60^{\circ} \mathrm{C}$ throughout. Samples were then immersed in the lead aspartate solution and kept at $60{ }^{\circ} \mathrm{C}$ for one hour. After three washing steps with double distilled water for 10 minutes each, the samples were dehydrated with a graded ethanol series starting with $30 \%$ over 50, 70 up to $100 \%$ with several exchanges in-between. Final dehydration prior to resin infiltration was facilitated with two exchanges of propylene oxide for 5 minutes each. Resin infiltration was mediated with a starting solution of 1:1 Epon resin and propylene oxide for one hour at room temperature followed by placing the samples into fresh $100 \%$ Epon resin for an additional hour. For overnight infiltration, the samples were placed again in fresh Epon resin on a rocker table over night at room temperature. The following day the samples were embedded in BEEM capsules and resin polymerisation took place over 48 hours at $60^{\circ} \mathrm{C}$.

\section{Adapted fBROPA protocol for transmission electron microscopy}

Cells were immobilized using a pre-warmed solution consisting of $2.5 \%$ glutaraldehyde in 0.1 M Sorensen's phosphate buffer at pH 7.4. Sample immobilization was continued for one hour at room temperature and was completed over night at $4{ }^{\circ} \mathrm{C}$. In case the supernatant turned yellowish in the process, the fixative was replaced with fresh solution. Secondary fixation was started using $1 \%$ osmium tetroxide in $1.5 \% \mathrm{~K}_{4}\left[\mathrm{Fe}(\mathrm{CN})_{6}\right]$ in $0.1 \mathrm{M}$ Sorensen's phosphate buffer for one hour at room temperature. Without any washing in-between, the solution was replaced with $1 \%$ osmium tetroxide in $0.1 \mathrm{M}$ Sorensen's phosphate buffer and incubated for an additional hour at room temperature. During three extensive washing steps using double distilled water for 10 minutes each, the Walton's lead aspartate solution was prepared. Therefore, $40 \mathrm{mg}$ aspartic acid were dissolved in double distilled water and the solution brought up to $60{ }^{\circ} \mathrm{C}$ in an oven. Next, $66 \mathrm{mg}$ lead nitrate were added to the solution. The $\mathrm{pH}$ of the mixture was then adjusted using $1 \mathrm{~N}$ sodium hydroxide solution. Care has to be taken that the mixture remained at $60^{\circ} \mathrm{C}$ throughout. Samples were then immersed in the lead aspartate solution and kept at $60^{\circ} \mathrm{C}$ for one hour. After three washing steps with double distilled water for 10 minutes each, the samples were dehydrated with a graded ethanol series starting with $30 \%$ over 50, 70 up to $100 \%$ with several exchanges in-between. Final dehydration prior to resin infiltration was facilitated with two exchanges of propylene oxide for 5 minutes each. Resin infiltration was mediated with a starting solution of 1:1 Epon resin and propylene oxide for one hour at room temperature followed by placing the samples into fresh $100 \%$ Epon resin for an additional hour. For overnight infiltration, the samples were placed again in fresh Epon 
resin on a rocker table over night at room temperature. The following day the samples were embedded in BEEM capsules and resin polymerisation took place over 48 hours at $60{ }^{\circ} \mathrm{C}$.

\section{Cell immobilization for high-pressure frozen cells}

For correlative imaging, HeLa cells were seeded in $10 \mathrm{~cm}$ cell culture dishes at a confluency of approximately $70 \%$. On the day of freezing, the cells were incubated with a final concentration of $200 \mathrm{~nm}$ Mitotracker Deep Red FM (Thermo Fisher Scientific) in DMEM complete medium (Thermo Fisher Scientific) for 1 hour. Cells were then detached by using $0.25 \%$ Trypsin in PBS and collected in a $15 \mathrm{ml}$ Falcon-tube. Trypsin was deactivated by adding the double volume of pre-warmed DMEM complete medium. Cells were then centrifuged at $300 \mathrm{~g}$ for 5 minutes. Cell pellets were re-suspended in DMEM without phenol red and were supplemented with a final concentration of HEPES at $25 \mathrm{mM}$, before being transferred to a 1.5 $\mathrm{ml}$ Eppendorf-tube. The cell suspension was kept in a heated metal block at $37^{\circ} \mathrm{C}$ throughout this process. Prior to freezing cells were concentrated with a table top centrifuge to achieve a paste-like consistency of the suspension. Small aliquots of about $2 \mu \mathrm{L}$ were transferred to goldcoated copper planchettes (Wohlwend) and immobilized by high pressure freezing in a Leica HPM100 (Leica Microsystems). The obtained frozen cell pellets were kept in liquid nitrogen until further use. These methods were published in (145).

\section{Freeze substitution and resin embedding for CLEM}

Sample blocks were obtained by freeze substitution of the cell pellets in $0.5 \%$ uranyl acetate diluted from a $20 \%$ stock in methanol and $3 \%$ double distilled $\mathrm{H}_{2} \mathrm{O}$ in acetone in an automated freeze substitution machine (Leica AFS1, Leica Microsystems). Temperature was set to -130 ${ }^{\circ} \mathrm{C}$ for the first 2 hours and then raised to $-90^{\circ} \mathrm{C}$ with a $20^{\circ} \mathrm{C} / \mathrm{hr}$ gradient. Pellets were then kept at $-90^{\circ} \mathrm{C}$ for 8 hours and temperature was then raised to $-45^{\circ} \mathrm{C}$ in a $5^{\circ} \mathrm{C} / \mathrm{hr}$ gradient. At $-45^{\circ} \mathrm{C}$ the pellets were washed three times with pre-cooled pure acetone over the course of 1.5 hours. For resin infiltration the pellets were incubated with 25, 50, 75 and $100 \%$ HM20 (Science Services) dilutions in pure acetone for 2 hours each. Pellets were kept in fresh 100 $\% \mathrm{HM} 20$ overnight while temperature was raised to $-25^{\circ} \mathrm{C}$ with a $5^{\circ} \mathrm{C} / \mathrm{hr}$ gradient. On the next day over the course of 8 hours, the resin was replaced three times with fresh $100 \% \mathrm{HM} 20$ resin. Pellets were then transferred to gelatine capsules and UV polymerized over the first 48 hours at $-25^{\circ} \mathrm{C}$, followed by a temperature rise to $0{ }^{\circ} \mathrm{C}$ with a $5{ }^{\circ} \mathrm{C} / \mathrm{hr}$ gradient. After trimming of the gelatine capsules, samples could be used immediately. However, I kept sample blocks at room temperature in the dark in a fume hood to complete polymerization for at least two days. Final sample blocks could easily be checked for fluorescence preservation with a 10x air 
objective. Alternatively, thick sections of $400 \mathrm{~nm}$ may be cut with a Histo-knife and observed with a fluorescence microscope. These methods were published in (145).

\section{High-accuracy post-embedding CLEM}

For high-accuracy CLEM, sections of $160 \mathrm{~nm}$ thickness were cut with a $35^{\circ}$ DiATOME ultra knife and collected on carbon-coated Formvar finder grids (Ted Pella 01910-F; Electron Microscopy Sciences). Grids were kept in the dark throughout the whole process and were observed as soon as possible. For fluorescence microscopy, the grids were placed on a glass microscope slide, covered with a drop of PBS and covered with a glass coverslip. Areas of interest were identified with a 20x air objective and detailed images acquired with a 100x oil immersion objective. Bright field images were acquired to allow for a re-identification of the areas, DAPI channel images were acquired to record uranyl acetate fluorescence, for easier re-identification of the cells. Finally, the channel of interest was imaged to reveal the protein/label. After imaging, the grids were recovered by pipetting fresh PBS on one side of the cover slips in order to float them off gently. Grids were then dried and stored until TEM investigations. Overview images were taken at an original magnification of $600 \mathrm{x}$ for identification of the cells of interest. Detailed images of grid boxes were taken in a tile scan of $10 \times 10$ images, with an original magnification of 3500x. Overview images of individual cells were obtained by merging the corresponding images with Photoshop CS6 using the "Photomerge" automated procedure. CLEM overviews were obtained by correlating the light microscopy data with the electron microscopy images in lcy with the plug-in eC-CLEM (152). These methods were published in (145).

\section{Image registration for CLEM}

Precise correlation of the data obtained by several imaging modalities could be achieved since all imaging techniques include characteristic features and intrinsic landmarks of the cells, without the need for additional fiducial markers (e.g. fluorescent beads). Image registration was carried out by monitoring a target point and landmarks evenly spread out throughout the field of view, until a minimal localization error was achieved for that point $2 \mathrm{D}$ or $3 \mathrm{D}$ linear transformation). For high-accuracy CLEM, the fluorescent data was up-scaled to match the electron micrograph. These methods were published in (145). 


\section{Cellular imaging}

\section{Transmission electron microscopy}

Parameters for imaging (section thickness, original magnification, operation of the microscope) in transmission electron microscopy are listed for every experiment individually. Electron micrographs were taken, if not stated otherwise, on a Philips CM120 transmission electron microscope equipped with a $\mathrm{LaB}_{6}$-source and a TVIPS $2 \times 2$ slow-scan CCD camera. These methods were adapted from (145)

\section{Epifluorescence imaging}

Fluorescence images for correlative microscopy were, if not stated otherwise, recorded on a Leica DM6000B microscope (Leica Microsystems $\mathrm{GmbH}$ ), equipped with a CCD-camera (DFC350FX) and the following filter cubes. A4 (UV): Exc 360/40, Dichro 400, Suppres BP 470/40. BGR (UV): Exc 420/30; 495/15; 570/20, Dichro 415; 510; 590, Suppres BP 465/20; 530/30; 640/40. GFP (blue): Exc 470/40, Dichro 500, Suppres BP 525/50. L5 (blue): Exc BP 480/40, Dichro 505, Suppres LP 527/30. N3 (green): Exc BP 546/12, Dichro 565, Suppres LP 600/40. SFRED (red): Exc HQ 630/20x, Dichro Q649LP, Suppres HQ 667/30. Furthermore, this microscope allows imaging in brightfield and phase contrast microscopy. 


\section{Results}

\section{Section 1 - Mitochondrial adaptation in the neuronal presynapse}

In section 1, the structure and specific adaptations of mitochondria in neuronal presynapses will be analysed. To this end, mitochondria in cancer cells and in 2D cultures of cultivated rat hippocampal neurons were visualized with transmission electron microscopy in different metabolic conditions of the cells. Finally, these mitochondrial structures were compared with the ultrastructure of presynapses in the cochlear nucleus of mice at different ages as an invivo model for mitochondrial adaptation to an increase in presynaptic energy demand.

\section{Development of high contrast staining procedures for electron microscopy}

Electron microscopy of cells requires the optimization of fixation and staining protocols. This ensures that the features of interest are well preserved and can be visualized in the electron micrographs. In the following, several staining procedures for electron microscopy were tested and compared. A central criterion for the comparison was the visibility of the inner mitochondrial membrane architecture. To test the standard preparation protocol, rat hippocampal neurons were cultivated for 21 DiV on PLL coated glass coverslips. After immobilization by aldehyde fixation and subsequent sample preparation for transmission electron microscopy, images of $70 \mathrm{~nm}$ thick sections, mounted on Formvar-coated slot-grids were recorded at $120 \mathrm{kV}$ with an original magnification of $6500 \mathrm{x}$.

Electron micrographs were subjected to a 2 pixel median filter in imageJ (153). Mitochondrial inner architecture can be visualized in mitochondria located in the soma (Figure 9 A). The mitochondrial ultrastructure in neurites and especially in presynapses is mostly obscured due to an electron-dense staining of the mitochondrial matrix. While in such cases the membranous structure can still be assessed due to a lighter intra-crista staining, the less ordered arrangement of crista-membranes in presynaptic mitochondria reduces visibility in electron micrographs significantly (Figure 9 F).

In contrast, other structures commonly visible in electron micrographs from neuronal cells like microtubules (Figure 9 D), synaptic vesicles (Figure 9 E and F) and the post-synaptic-density (Figure 9 F) are clearly detectable and easy to identify. Therefore, the standard staining procedure results in a good preparation of the hippocampal neurons for proper visualization of the overall cell morphology and many ultrastructural details. 

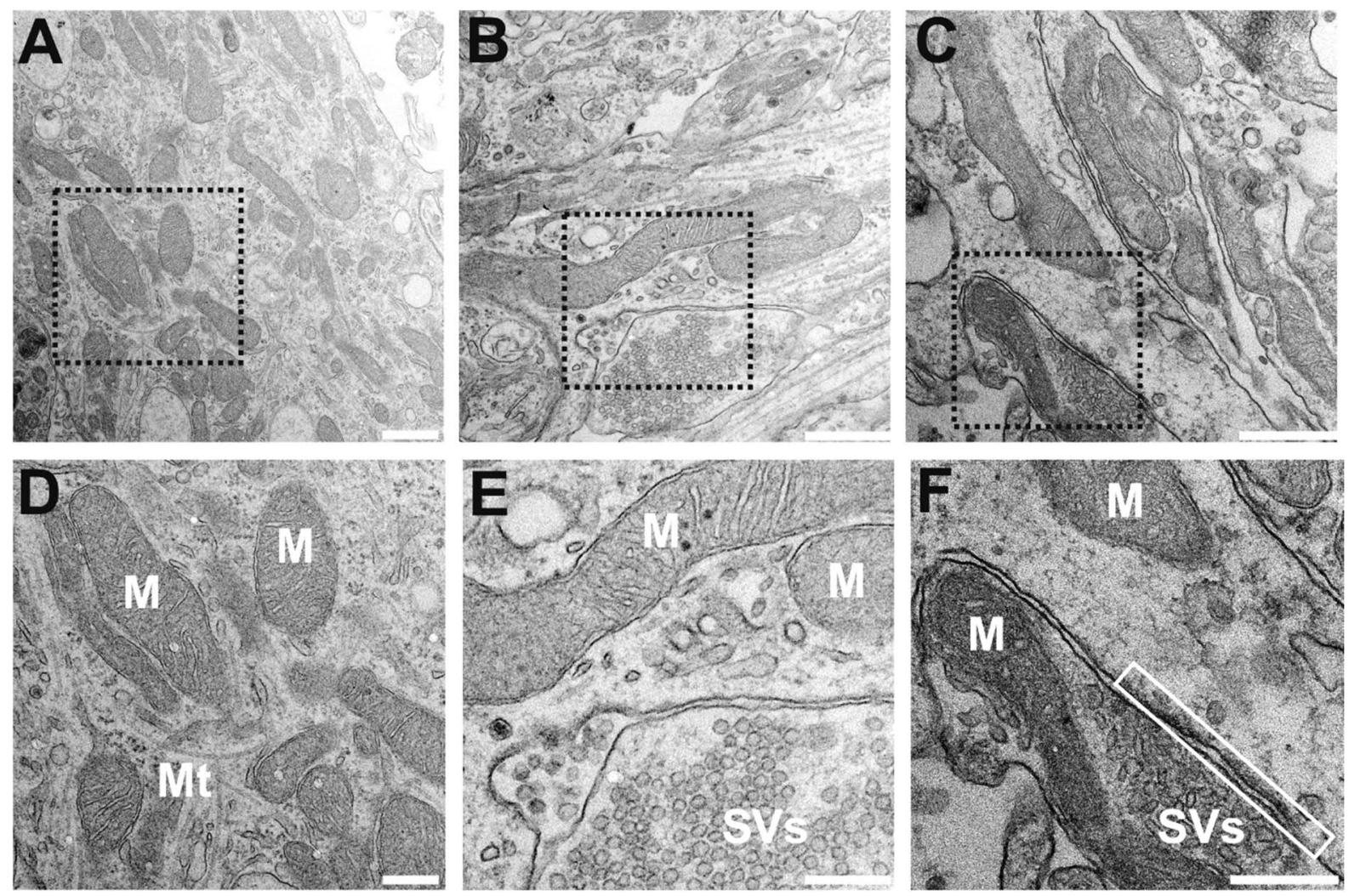

Figure 9: Transmission electron microscopy images of rat hippocampal neurons prepared with the standard protocol. A: Mitochondria located in the soma. B: Mitochondria located in dendrites. $\boldsymbol{C}$ : Mitochondria located in dendrites and presynapses. $\boldsymbol{D}, \boldsymbol{E}$ and $\boldsymbol{F}$ show detailed view of dot-line boxes from the upper row. $\boldsymbol{M}=$ mitochondria. $\boldsymbol{M t}=$ microtubules. $\mathbf{S} \boldsymbol{V} \boldsymbol{s}=$ synaptic vesicles. White box in $F=$ postsynaptic density. Scale bars in $A, B$ and $C=500 \mathrm{~nm}$. Scale bars in $D, E$ and $F=200 \mathrm{~nm}$.

However, it is not sufficient to provide clear representations for internal mitochondrial membrane arrangements. For a more detailed analysis of the ultrastructural features of these mitochondria, an adapted protocol is necessary. In the following section, different adaptations of protocols for cellular staining in electron microscopy will be compared for cultured cancer cells as well as isolated rat hippocampal neurons.

\section{High contrast membrane procedure}

For a high contrast staining of cellular membranes, an additional staining with $\mathrm{K}_{4}\left[\mathrm{Fe}(\mathrm{CN})_{6}\right]$ reduced osmium tetroxide was employed prior to secondary fixation. The oxidation state of $\mathrm{Os}^{\mathrm{i} v}$ is more hydrophobic and therefore solubilizes in biological membranes increasing the resulting contrast in electron microscopy of these structures (154). Additionally, samarium trichloride for mitochondrial membrane staining was tested as it was shown to enrich on mitochondrial membranes (115). To evaluate the overall efficiency of the protocol, HeLa cells were treated the same way. Rat hippocampal neurons were cultivated for $21 \mathrm{DiV}$ on glass coverslips. HeLa cells were seeded on Aclar coverslips at approximately $70 \%$ confluency. 
After immobilization by CAF and subsequent sample preparation for transmission electron microscopy, images of $60 \mathrm{~nm}$ thick sections, mounted on Formvar-coated slot-grids were recorded at $120 \mathrm{kV}$ with an original magnification of $6500 \mathrm{x}$. Electron micrographs were subjected to a 2-pixel median filter in imageJ. The appearance of cellular components changed dramatically compared with the standard protocol (Figure 10). The nucleus appears only lightly stained and very homogenous (Figure $10 \mathrm{~A}$ ). The nuclear envelope appears as a dark structure delineating the chromatin as opposed to standard preparations. Also round and very dark structures are visible that can be presumably assigned to lysosomal-compartments. The mitochondrial membranes were more clearly visible than with the standard preparation procedure.
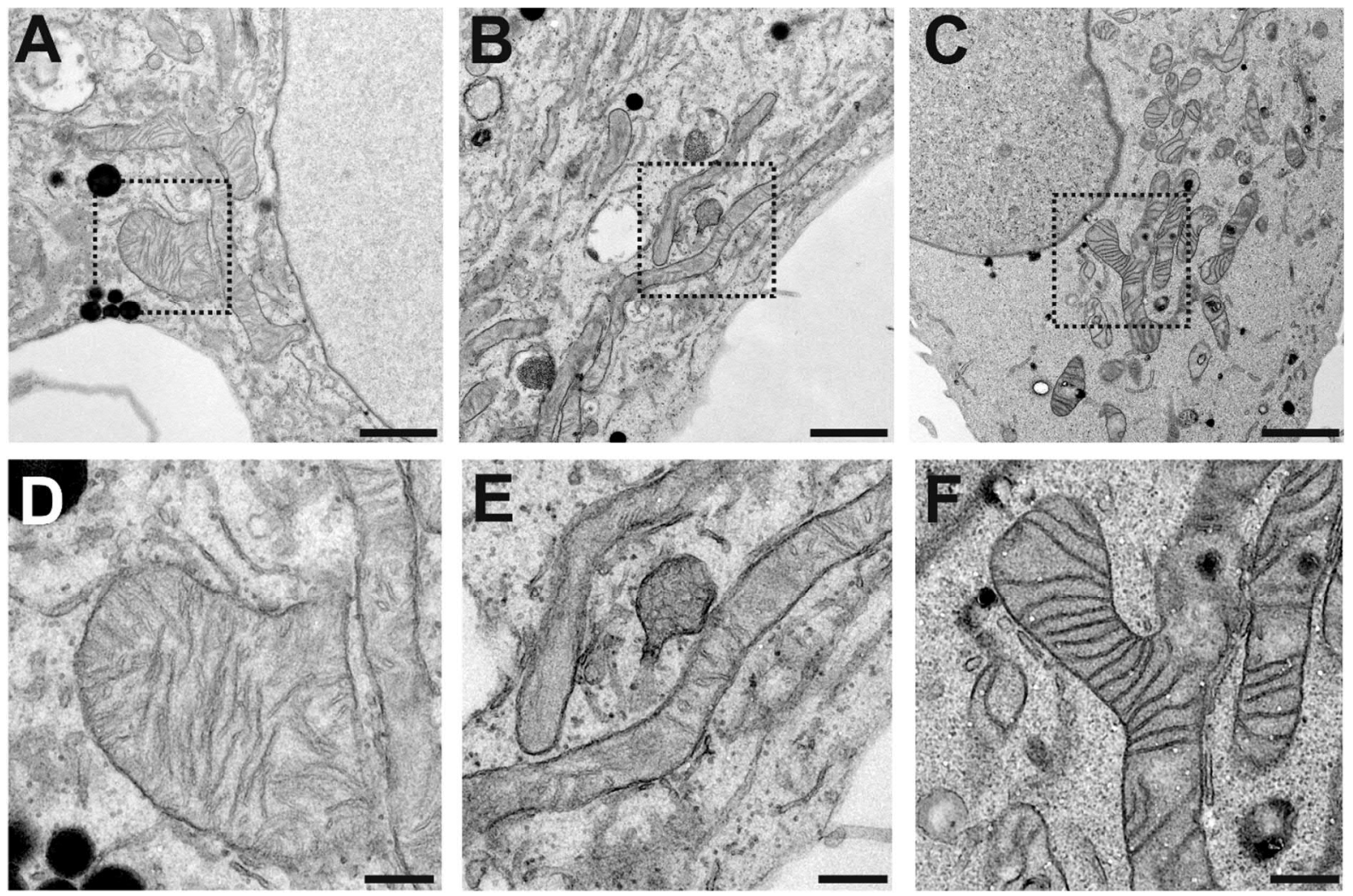

Figure 10: Transmission electron micrographs of rat hippocampal neurons and HeLa cells suing the high-contrast protocol. A, B: Soma of rat hippocampal neurons. $\boldsymbol{C}$ : Cytoplasm of a HeLa cell. $\boldsymbol{D}, \boldsymbol{E}$ and $\boldsymbol{F}$ show boxed regions from $A, b$ and $C$ respectively. Scale bars for $A, B$ and $C=500 \mathrm{~nm}$. Scale bars for $D, E$ and $F=200 \mathrm{~nm}$.

The matrix is more lightly stained while the membrane staining of all organelles is more pronounced. In neuronal cells however, the mitochondrial outer membrane shows the most enrichment in stain and thus appears darker than, for example, the crista-membranes (Figure $10 \mathrm{E})$. Structures like the cytoskeleton are not visible in this preparation. Protein rich structures located in the cytoplasm, as for example ribosomes, are visible due to their enriched staining. This is not the case in this preparation; the cytoplasm appears more homogeneously stained. 
In the comparative preparation of HeLa-cells, the structure of the organelles can be readily assessed in low magnifications due to the pronounced staining of the membranes. The nucleus shows a uniform staining. Eu- and heterochromatin were not distinguishable. The nuclear envelope appears as a dark delineation of the chromatin as well. Thus, this protocol stained primarily lipidic structures such as membranes. All organelle membranes in HeLa cells are equally enriched in stain and thus very well visible.

The high contrast protocol provided enhanced staining of organelle membranes in HeLa cells. For neuronal cells, the application of the protocols also resulted in an increased contrast of the membranes in mitochondria and less electron-dense staining of the mitochondrial matrix. However, the mitochondrial outer membrane of neuronal mitochondria was more enriched in stain but crista membranes are still not clearly visualized. To analyse the crista membranes, the contrast of these membranes needs to be further improved. This could be achieved with additional labelling of membranous structures with heavy metals.

\section{fBROPA protocol for enhanced membrane staining}

The "fast brain-wide reduced-osmium staining with pyrogallol-mediated amplification" protocol (fBROPA) was recently employed for the improved staining of organ-pieces for volume-SEM image acquisitions (155). As a main difference to existing protocols, this approach features the use of pyrogallol instead of thio-carbohydrazide and Walton's lead aspartate staining instead of en-bloc staining with uranyl acetate. In both cases, whether using pyrogallol or thiocarbohydrazide, a multilayer of osmium-crosslinking products on biological and organelle membranes is deposited resulting in a strong accentuation of membranes within the sample while reducing the visibility of any cytosolic and matrix localized features.

Here it was tested if the fBROPA protocol improves imaging of neuronal mitochondria with electron microscopy. To this end, rat hippocampal neurons were cultivated for $21 \mathrm{DiV}$ on glass coverslips. HeLa-cells were seeded on ACLAR coverslips at approximately $70 \%$ confluency. The cells were then processed and resin embedded in Epon according to the fBROPA protocol. Images of $50 \mathrm{~nm}$ thick sections, mounted on Formvar-coated slot-grids, were recorded at 120 $\mathrm{kV}$ with an original magnification of $6500 x$. Electron micrographs were subjected to a 2-pixel median filter in imageJ.

Exemplary images of rat hippocampal neurons and HeLa cells were obtained after staining with the fBROPA protocol (Figure 11). Structures as the nucleus appeared lightly stained and homogeneous with the nuclear envelope as a black line delineating the chromatin from the cytoplasm (for example see Figure $11 \mathrm{~A}$ ). In addition, sheets of the endoplasmic reticulum appeared almost uniformly filled as black lines. As opposed to the procedures described 
previously in this section, the mitochondrial inner architecture in both HeLa cells and neurons was visible already in low original magnifications (Figure 11 A and B). The mitochondrial matrix showed almost no staining which contributed to the good contrast of the cristamembranes. The cytoskeleton was invisible in the soma but could be visualized when highly abundant like in neurites (Figure $11 \mathrm{E}$ ).
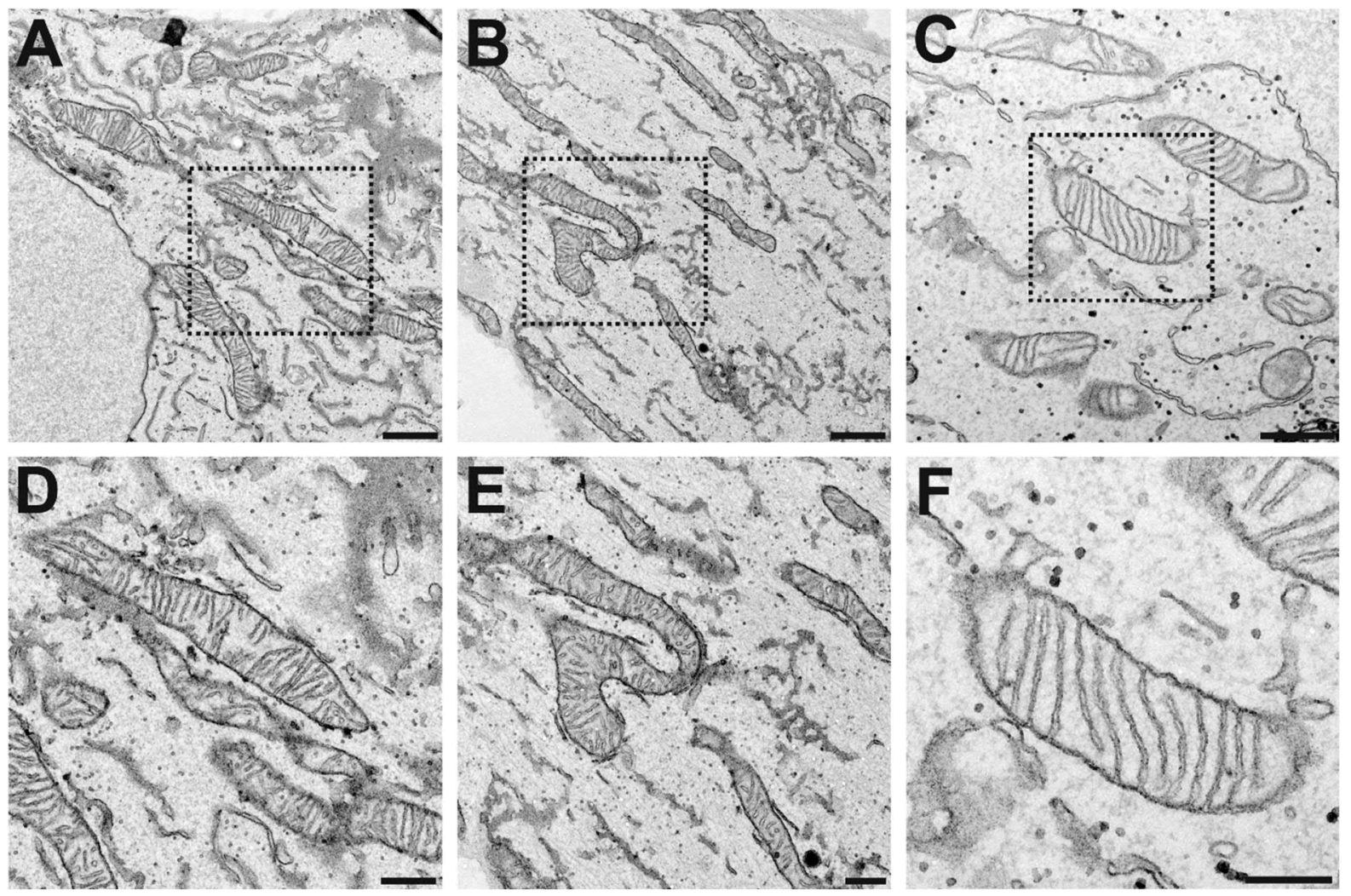

Figure 11: fBROPA protocol preparations of rat hippocampal neurons and HeLa cells. $\boldsymbol{A}$ and B: Soma of rat hippocampal neurons. C: Cytoplasm of a HeLa-cell. D, $\boldsymbol{E}$ and $\boldsymbol{F}$ show boxed regions in $A, B$ and $C$ respectively. Scale bars for $A, B$ and $C=500 \mathrm{~nm}$. Scale bars for $D, E$ and $F=200 \mathrm{~nm}$.

Proteins in the cytoplasm were not visible with this preparation technique. In HeLa-cells, the organelles appear a bit clearer as in the preparations from neuronal cells. However, at higher magnifications, the structure of the mitochondrial membranes shows some aberrations, which might be caused by the incubation with lead aspartate at higher temperatures. The multilayer deposition of heavy metals will result in an increased thickness of lipid bilayers. Using this protocol, the thickness of the ultra-thin sections should not exceed $50 \mathrm{~nm}$ as otherwise the membranous structures will appear less defined within the electron micrographs and can overlap with each other (Figure 11 F).

The mitochondrial ultrastructure in rat hippocampal neurons and HeLa cells could be visualized with high contrast of all membranes. Further, in mitochondria of neuronal cells no electron- 
dense staining of the mitochondrial matrix was visible. However, multiple layers of heavy metal stain decrease the resolution that can be obtained at higher magnifications.

The application of the fBROPA protocol can reduce some of the problems of caused by poor membrane staining of organelles when analysing neuronal cells. However, it is also evident, that the deposition of multiple layers of osmium onto organelle membranes led to a significant decrease of resolution in transmission electron microscopy, especially when imaging at higher original magnifications. Taken together from the previous protocol establishments, the membrane contrast can be increased by using a series of reduced osmium and regular osmium staining without compromising resolution too much. Furthermore, the replacement of uranyl acetate for Walton's lead aspartate for en-bloc staining results in a less stained mitochondrial matrix thus, leading to a better visibility of the crista membranes. For a detailed analysis of presynaptic mitochondria, an adaptation of this protocol is needed. Avoiding multiple depositions of osmium on membranes as an adaptation of the fBROPA protocol might lead to an improved resolution of cellular structures.

\section{Adapted high contrast protocol for neuronal cells}

In the adaptation of the fBROPA protocol, the deposition of multiple osmium layers on membranous structures was avoided. Rat hippocampal neurons were cultivated for $21 \mathrm{DiV}$ on glass coverslips. Alongside, HeLa-cells were seeded on Aclar coverslips at approximately 70 $\%$ confluency. After immobilization by CAF and subsequent sample preparation for transmission electron microscopy, images of $60 \mathrm{~nm}$ thick sections, mounted on Formvarcoated slot-grids, were recorded at $120 \mathrm{kV}$ with an original magnification of 6500x. Electron micrographs were subjected to a 2-pixel median filter in imageJ.

Membranes of organelles in rat hippocampal neurons and HeLa cells were clearly stained (Figure 12). All organelles could be distinguished easily. In addition, the inner mitochondrial architecture of somatic mitochondria was clearly visualized. This posed a significant improvement to the standard preparation protocol. However, cytoskeleton and smaller proteins were mostly obscured and could not be analysed properly with this preparation protocol. 

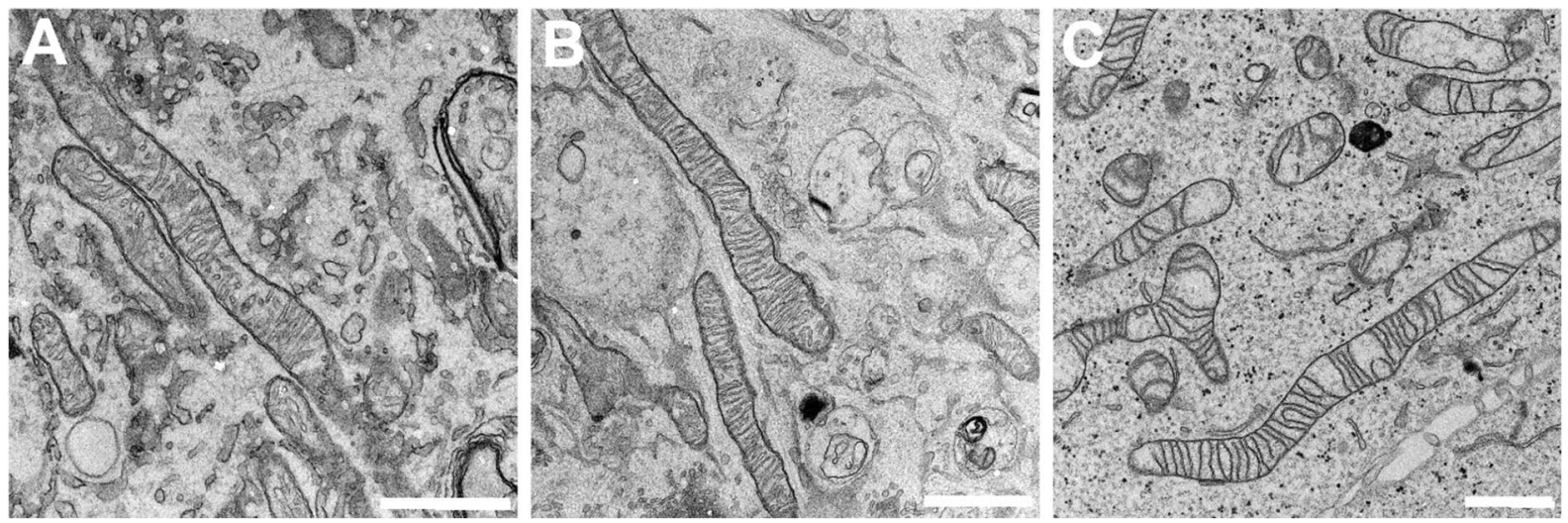

Figure 12: Adapted high contrast protocol preparations of rat hippocampal neurons and HeLa cells. Transmission electron micrographs show exemplary images of mitochondria found in both samples. A and B: Soma of rat hippocampal neurons. C: Cytoplasm of a HeLa cell. Scale bars $=500 \mathrm{~nm}$.

The adaptation of the fBROPA protocol resulted in a good visualization of mitochondrial ultrastructure from neurons and HeLa cells. Organelle membranes were sufficiently stained to image cellular structures at low magnification to obtain large overviews with contextual information of both cell types. Further, the ultrastructure of mitochondria was clearly visible at lower magnifications thus; multiple mitochondria could be captured in a single micrograph. The membranes of organelles appeared defined and neighbouring structures did not overlap with each other. For a detailed analysis at high magnifications, this was a significant improvement to the fBROPA protocol.

In summary, preparations of cells according to the standard procedure allowed staining organelle membranes and protein-rich structures in the cytoplasm. A good assessment of the cellular features was possible in HeLa cells. However, the precise architecture of mitochondria could not be visualized in neuronal cells. The high contrast protocol resulted in increased staining of organelle membranes but details such as cytoskeleton structures are not stained and thus, are not visualized. Further, the mitochondrial inner architecture of neuronal cells cannot be analysed in detail because the inner mitochondrial membranes are less enriched in stain compared to the outer mitochondrial membrane. Preparations using the fBROPA protocol resulted in strong staining of all organelles. Membranes could be visualized with high contrast. However, structures in close proximity overlap with each other and appeared less defined. The adaptation of the fBROPA protocol resulted in strong staining of organelle membranes but the overlap of structures was avoided. However, some contextual information was lost due to the insufficient staining of protein-rich structures. 
Table 1: Advantages and downsides of sample preparation protocols for transmission electron microscopy of neuronal mitochondria in the soma. Table 1 compares cellular features and their staining with respect to the protocols established in this thesis. Standard protocol preparations could be applied to visualize the general cell structures of neurons with limited visualization of the mitochondrial architecture. For imaging of neuronal mitochondria, the adapted high contrast protocol provided the best results.

\begin{tabular}{|l|c|c|c|c|}
\hline & $\begin{array}{c}\text { Standard } \\
\text { protocol }\end{array}$ & $\begin{array}{c}\text { High contrast } \\
\text { protocol }\end{array}$ & fBROPA & $\begin{array}{c}\text { Adapted high } \\
\text { contrast protocol }\end{array}$ \\
\hline $\begin{array}{l}\text { Membrane } \\
\text { staining }\end{array}$ & + & ++ & +++ & +++ \\
\hline Protein staining & +++ & - & - & - \\
\hline contrast & + & ++ & +++ & +++ \\
\hline Resolution & +++ & + & - & + \\
\hline $\begin{array}{l}\text { Visibility of } \\
\text { mitochondrial } \\
\text { architecture }\end{array}$ & -- & - & ++ & +++ \\
\hline
\end{tabular}

Several staining protocols for electron microscopy were tested on HeLa cells and neurons. For neuronal cells, different protocols have to be applied depending on the structure of interest that will be analysed. For a general overview of the cells, the standard preparation protocol produces good results. However, for the analysis of mitochondrial architecture, the adaptation of the fBROPA protocol yields better results compared to the standard protocol (Table 1).

\section{Preliminary experiments towards mitochondrial adaptation during metabolic switch}

Mitochondria form a flexible network that can adjust to meet cellular energy demands. The first section described multiple sample preparation protocols to visualize the mitochondrial ultrastructure in electron microscopy. These protocols were then applied to study the mitochondrial structure in several cancer cell lines. For the analysis of the performance of these organelles in the cellular context, chemical inhibitors of the respiratory chain complexes can be used to modify the mitochondrial activity and to reduce the amount of ATP that can be provided. Such inhibitors can also have strong undesirable effects on the cellular activity. The functions of mitochondrial proteins are generally interdependent and the inhibition of a protein complex might have numerous side effects. In order to avoid possible stress reactions of the cells to a chemical inhibitor, the plasticity of mitochondria was analysed using a metabolic switch of the cells.

For this purpose, the long-term adaptation of Hela, U-2 OS and Cos7 to the availability of different carbon sources. The availability of different carbon sources might switch the cell 
metabolism to either glycolysis or mitochondrial respiration as a main source for ATP. This might further affect the mitochondrial architecture.

\section{Structural adaptations of mitochondria in presence of different carbon substrates in cultivated cancer cell lines}

Cultured cancer cell lines were used in a preliminary experiment to study the impact of a certain energy substrate to the mitochondrial ultrastructure. Culture medium containing $30 \mathrm{mM}$ glucose was used as the control condition. Further, a lower concentration of glucose was tested. Sodium-pyruvate and beta-hydroxybutyrate can only be utilized by mitochondrial respiration to generate ATP in the cells while ATP generation via glycolysis is avoided in these test conditions. Therefore, cells grown in presence of either sodium-pyruvate or betahydroxybutyrate may switch their metabolism towards higher activity of oxidative phosphorylation.

To analyse the mitochondrial adaptation on a structural level, HeLa CCL, U-2 OS and Cos7 cells were seeded on ACLAR slides in a $6 \mathrm{~cm}$ cell culture dish at a confluency of approximately $30 \% .24$ hours later the dishes were briefly washed with warm PBS to minimize the amount of residual DMEM complete medium and transferred into pre-warmed culture medium only containing the selected carbon substrates.

Table 2: Vitality of cells grown in DMEM for three days. Culture medium supplemented with either $30 \mathrm{mM}$ glucose, $5 \mathrm{mM}$ glucose, $10 \mathrm{mM}$ sodium-pyruvate or $25 \mathrm{mM}$ beta-hydroxybutyrate respectively without supplementation of serum. The vitality of the cultures was qualitatively evaluated using phase-contrast light microscopy. Red boxes indicate cultures where almost all cells were dead and detached (indicated with --). Yellow boxes indicate cultures with higher amounts of detached cells (indicated with -). Light green boxes indicate cell cultures that are vital but showed few detached cells (indicated with a + ). Green boxes indicate cell cultures with high vitality (indicated with ++ ).

\begin{tabular}{|c|c|c|c|}
\cline { 2 - 4 } \multicolumn{1}{c|}{} & HeLa CCL & U-2 OS & Cos7 \\
\hline $30 \mathrm{mM}$ glucose & + & + & ++ \\
\hline $\mathbf{5} \mathrm{mM}$ glucose & ++ & ++ & - \\
\hline $10 \mathrm{mM}$ pyruvate & - & + & - \\
\hline $25 \mathrm{mM}$ bHB & -- & -- & - \\
\hline
\end{tabular}

The cells were then cultivated in DMEM no-glucose supplemented with either $30 \mathrm{mM}$ glucose, $5 \mathrm{mM}$ glucose, $10 \mathrm{mM}$ sodium-pyruvate or $25 \mathrm{mM}$ beta-hydroxybutyrate respectively for three days without supplementation of fetal calf serum. On every day, the condition of the cultures was checked using phase-contrast light microscopy (Table 2). Green boxes indicate a healthy culture. Light green boxes indicate cultures in good condition with only minimal cell 
detachment. Yellow boxes indicate a culture showing increased rates of cell detachment and dead cells in the supernatant. Red boxes indicate cultures with a few up to no attached cells present on the coverslip.

After three days of cultivation, the cells were immobilized with a fresh prepared solution containing $2.5 \%$ glutaraldehyde in $0.1 \mathrm{M}$ Sorensen phosphate buffer ( $\mathrm{pH} 7.4$ ) pre-warmed to $37^{\circ} \mathrm{C}$. After one-hour incubation at room temperature, the cells were transferred to $4{ }^{\circ} \mathrm{C}$ and the fixation completed overnight. Secondary fixation, staining and final resin embedding was carried out according to the high-contrast protocol. Ultra-thin sections of $60 \mathrm{~nm}$ thickness were examined at $120 \mathrm{kV}$ with $8600 \mathrm{x}$ original magnification. The sections were screened for cells that appeared healthy and without any visible signs of cellular stress.

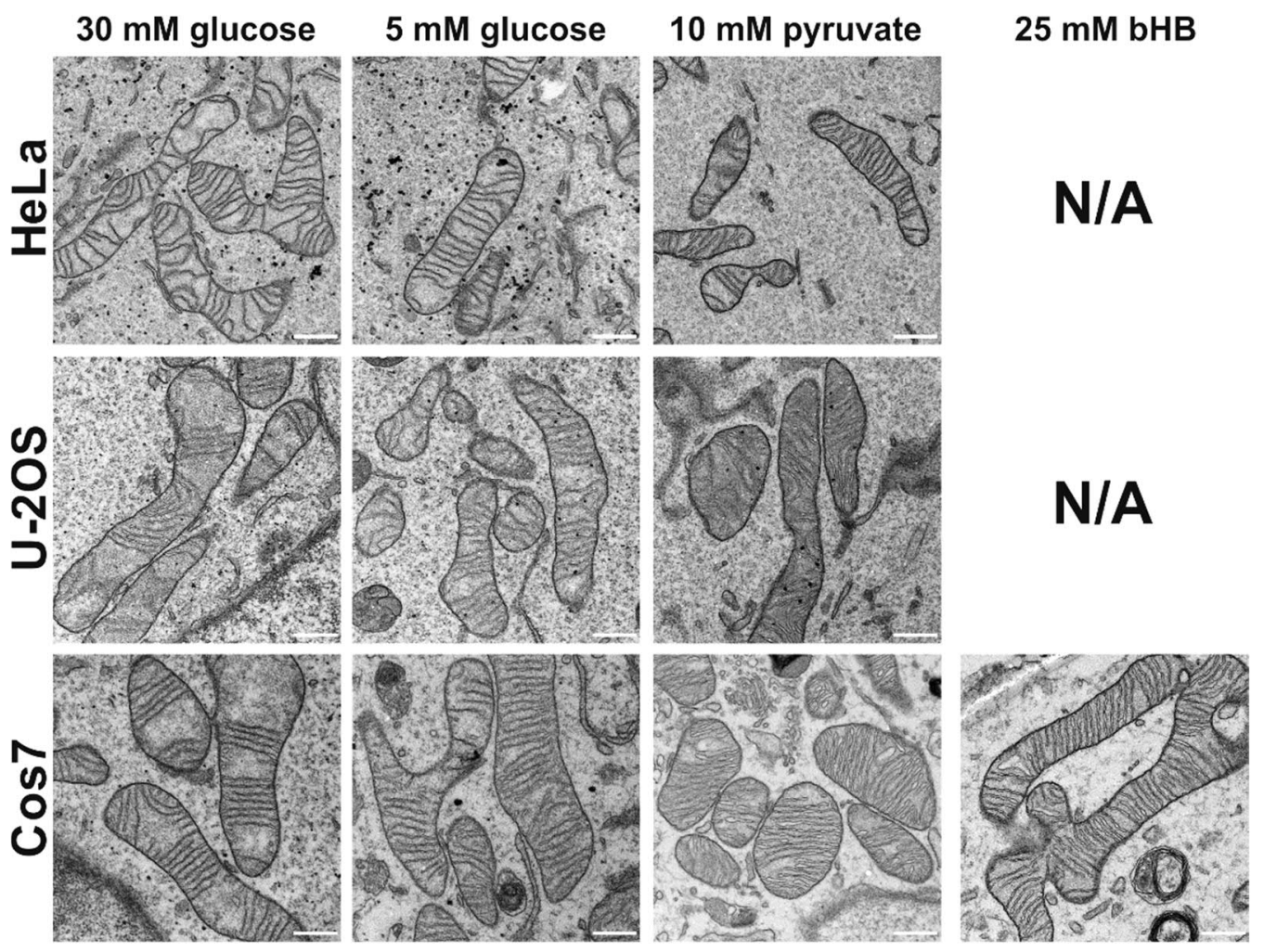

Figure 13: Transmission electron microscopy images of mitochondria from HeLa, U-2 OS and Cos 7 cells. Cells were grown for three days in DMEM in presence of either $30 \mathrm{mM}$ glucose, 5 $\mathrm{mM}$ glucose, $10 \mathrm{mM}$ sodium-pyruvate or $25 \mathrm{mM}$ beta-hydroxybutyrate without supplementation of serum. Cultures of HeLa and U-2 OS cells cultivated in presence of $25 \mathrm{mM}$ betahydroxybutyrate could not be analysed in electron microscopy due to excessive cell death (indicated with N/A). Scale bars $=200 \mathrm{~nm}$.

The membranous ultrastructure of the mitochondria was only slightly affected when the energy substrate is changed over a long period. Mitochondria in all cell lines exhibited the lamellar crista-arrangement perpendicular to the mitochondria tube with a high rate of membrane stacking within every condition tested (Figure 13). The viability after three days of cultivation differs between the selected cell lines. While all cell lines seemed to tolerate the cultivation in 
glucose during serum starving, other conditions like cultivation in beta-hydroxybutyrate lead to an increase in cell detachment and cell death.

In conclusion, serum starving over three days might have already interfered substantially with the cell metabolism. Cell proliferation might be increased in culture conditions with sodiumpyruvate or beta-hydroxybutyrate supplemented as a carbon source. Thus, an increase in cell detachment due to the lack of nutrients is possible. In this experimental setup, a mitochondrial adaptation could not be analysed as the rates of cellular detachment and cell death were increased. An adaptation of the culture conditions was needed to avoid stress reactions of the cells to the depletion of growth factors. For subsequent experiments, a specialized formulation of DMEM was to be used.

\section{Structural adaptations of mitochondria in presence of different carbon substrates in cultivated cancer cell lines in specialized medium}

Cancer cells can switch to other substrates than glucose in order to fuel mitochondrial respiration. Especially glutamine might be used extensively for energy production in cancer cells compared to regular cells and tissues (156). Because of that, DMEM medium containing glutamine may not result in a specific adaptation of the mitochondrial structure to a change in carbon source. Therefore, in the following experiments the DMEM medium was exchanged to SILAC FLEX medium that is devoid of glutamine and any carbon source. The SILAC FLEX medium was then further supplemented with dialyzed fetal bovine serum to avoid serum starving of the cells. In line with previous experiment, cells were first plated in DMEM complete medium on Aclar sheets.

Table 3: Vitality of cells grown in SILAC FLEX medium without glutamine. Culture medium supplemented with either $30 \mathrm{mM}$ glucose, $10 \mathrm{mM}$ sodium-pyruvate or $25 \mathrm{mM}$ betahydroxybutyrate respectively. Conditions of the cultures were qualitatively evaluated using phase-contrast light microscopy. Light green boxes indicate cell cultures that are vital but showed few detached cells (indicated with $a+$ ). Green boxes indicate cell cultures with high vitality (indicated with ++ ).

\begin{tabular}{|c|c|c|c|}
\cline { 2 - 4 } \multicolumn{1}{c|}{} & HeLa CCL & U-2 OS & Cos7 \\
\hline $\mathbf{3 0} \mathrm{mM}$ glucose & + & + & + \\
\hline $\mathbf{1 0} \mathrm{mM}$ pyruvate & ++ & ++ & ++ \\
\hline $25 \mathrm{mM}$ bHB & ++ & ++ & ++ \\
\hline
\end{tabular}

To test the metabolic adaptation, the cells were then briefly washed with pre-warmed PBS ( $\mathrm{pH}$ 7.4) and subsequently transferred to pre-warmed SILAC FLEX medium supplemented with 
dialyzed serum and further supplemented with either $30 \mathrm{mM}$ glucose, $10 \mathrm{mM}$ sodium-pyruvate or $25 \mathrm{mM}$ beta-hydroxybutyrate respectively and cultivated for three days.

On each day, the vitality of the cell cultures was examined each day using phase-contrast light microscopy. Green boxes indicate cultures in good condition. Light green boxes indicate cultures with small amounts of detached cells (Table 3).

The cells were then immobilized with a freshly prepared solution containing $2.5 \%$ glutaraldehyde in $0.1 \mathrm{M}$ Sorensen phosphate buffer $(\mathrm{pH} 7.4)$ pre-warmed to $37^{\circ} \mathrm{C}$. The cells were then transferred to $4{ }^{\circ} \mathrm{C}$ and the fixation completed over night. Secondary fixation, staining and final resin embedding was carried out according to the high-contrast protocol. Ultra-thin sections of $60 \mathrm{~nm}$ thickness were examined at $120 \mathrm{kV}$ with $8600 \mathrm{x}$ original magnification (Figure 14).

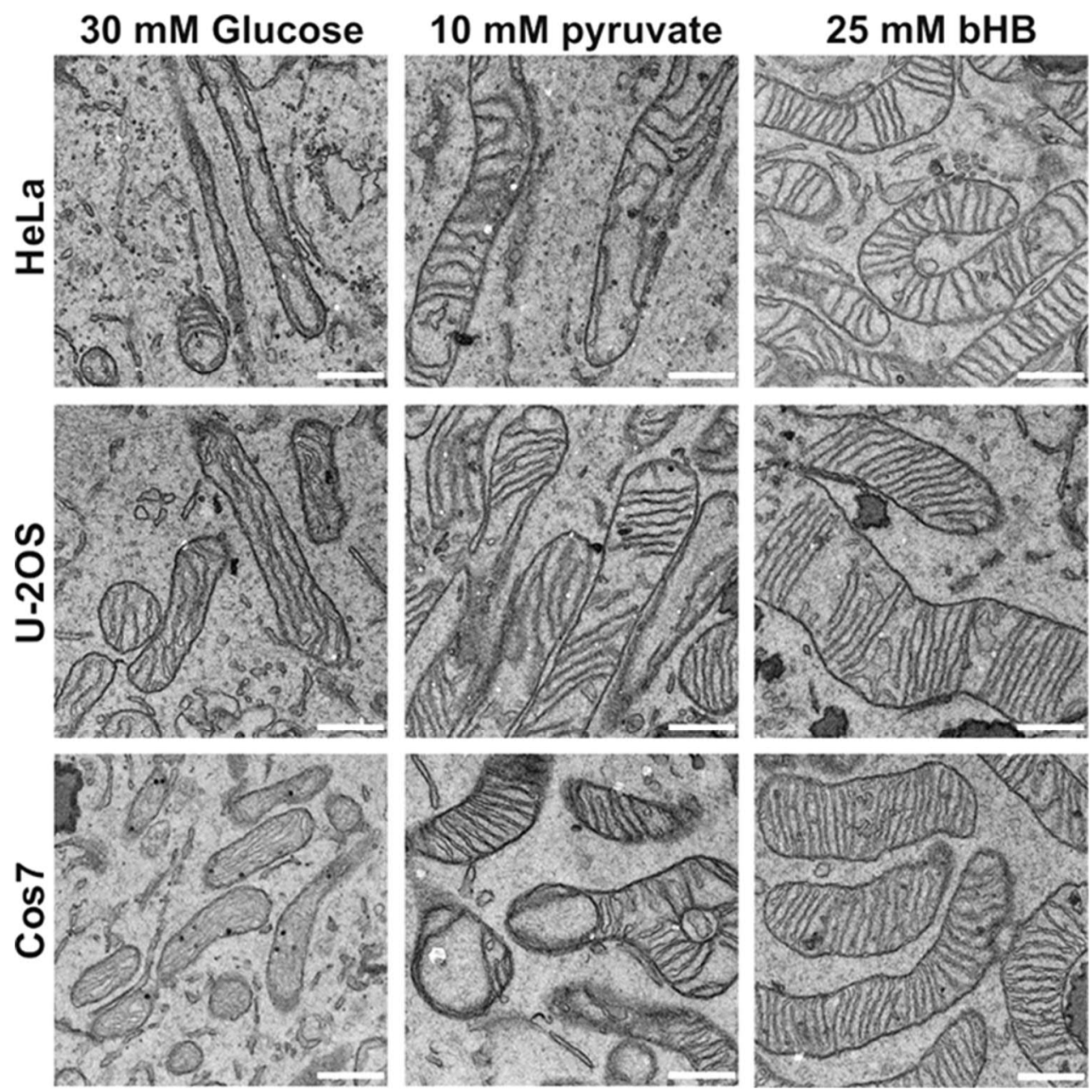

Figure 14: Transmission electron micrographs of HeLa, U-2 OS and Cos7 cells grown in SILAC FLEX with dialyzed serum and without glutamine. Cells were grown in medium with 30 $\mathrm{mM}$ glucose, $10 \mathrm{mM}$ sodium-pyruvate or $25 \mathrm{mM}$ beta-hydroxybutyrate respectively as energy substrates available for 3 days of cultivation. Scale bars $=200 \mathrm{~nm}$. 
Images of 15 to 20 cells each showing multiple cross-sections of mitochondria were then analysed. First, the diameter of the mitochondria was determined in imageJ (Figure 15).

Precisely, for HeLa cells grown in $30 \mathrm{mM}$ glucose, the average mitochondrial diameter was $201.1 \pm 54.5 \mathrm{~nm}$ (mean $\pm \mathrm{SD}, 243$ mitochondria). After cultivation in presence of $10 \mathrm{mM}$ sodium-pyruvate, the diameter increased to $285.6 \pm 82.7 \mathrm{~nm}$ (mean $\pm \mathrm{SD}, 254$ mitochondria; $\mathrm{p}<0.001$ ). With cultivation in presence of $25 \mathrm{mM}$ beta-hydroxybutyrate, the diameter increased to $298.3 \pm 66.9 \mathrm{~nm}$ (mean $\pm \mathrm{SD}, 162$ mitochondria; $\mathrm{p}<0.001, \mathrm{p}=0.1$ to cultivation in sodiumpyruvate). Cos7 cells cultivated in $30 \mathrm{mM}$ glucose had an average diameter of $220.2 \pm 59.8$ $\mathrm{nm}$ (mean $\pm \mathrm{SD}, 186$ mitochondria). After cultivation in presence of $10 \mathrm{mM}$ sodium-pyruvate, the diameter increased to $339.3 \pm 99 \mathrm{~nm}$ (mean $\pm S D$, 171 mitochondria, $p<0.001$ ).
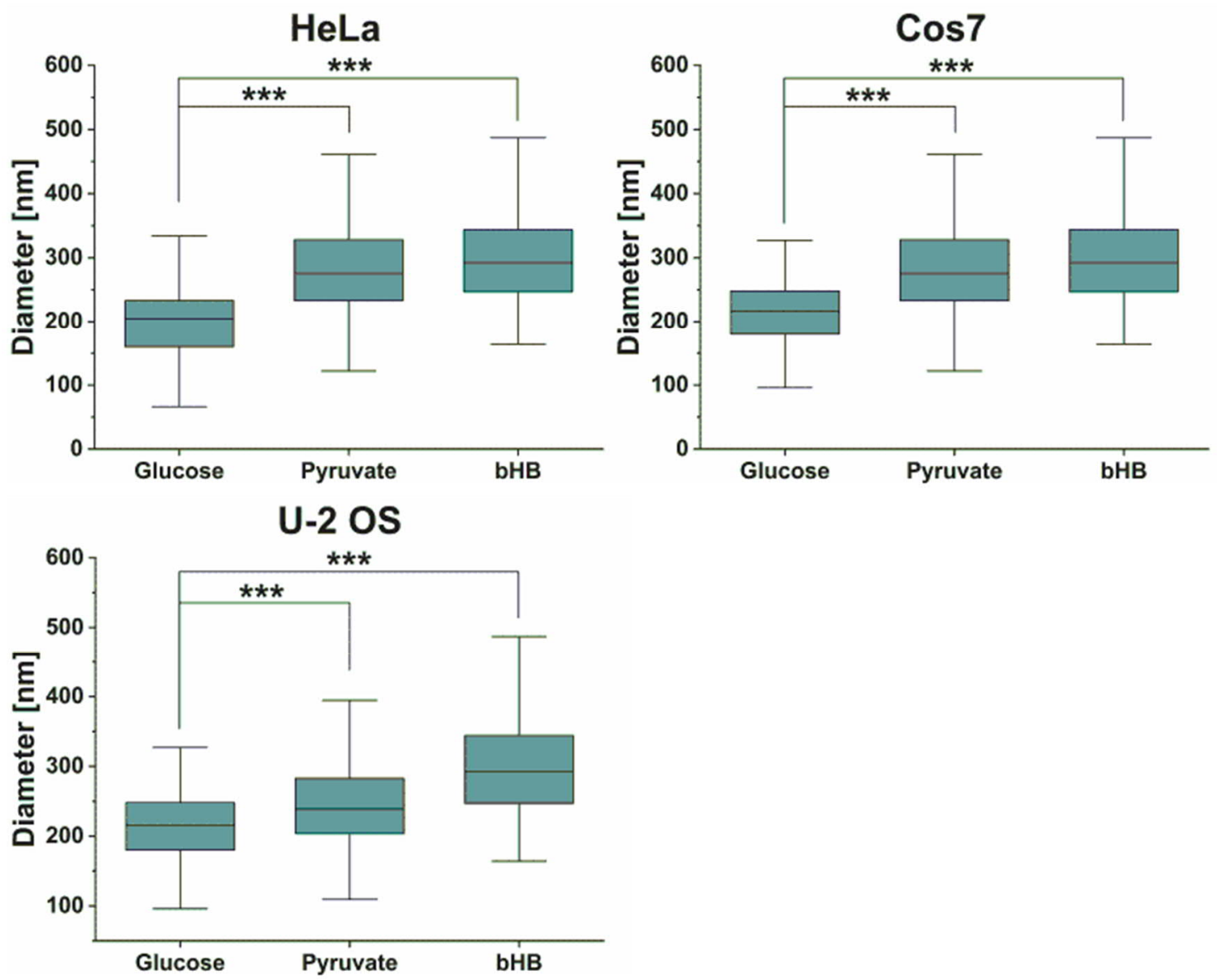

Figure 15: Morphological adaptations of mitochondria to a specific energy substrate in HeLa, Cos7 and U-2 OS cells. Statistical analysis of mitochondrial diameter for each condition picked from three individual experiments for each cell line and the substrate used. Box and whiskers plots show the mitochondria diameter obtained by manual measurements. The boxes indicate $25^{\text {th }}$ to $75^{\text {th }}$ percentile. The horizontal lines indicate median. Whiskers indicate $S D$. ${ }^{* * *} p<0.001$. After cultivation in presence of $25 \mathrm{mM}$ beta-hydroxybutyrate, the diameter increased to 361.1 $\pm 96.871 .8 \mathrm{~nm}$ (mean $\pm \mathrm{SD}, 142$ mitochondria, $p<0.001 ; p=0.051$ to cultivation in sodiumpyruvate). U-2 OS cells showed an average diameter of $204.6 \pm 51.7 \mathrm{~nm}$ (mean $\pm \mathrm{SD}, 156$ 
mitochondria) when grown in presence of $30 \mathrm{mM}$ glucose. After cultivation in presence of 10 $\mathrm{mM}$ sodium-pyruvate, the diameter increased to $248.7 \pm 71.8 \mathrm{~nm}$ (mean $\pm \mathrm{SD}, 224$ mitochondria; $p<0.001)$. The diameter further increased after cultivation in presence of 25 $\mathrm{mM}$ beta-hydroxybutyrate to $277.7 \pm 62.3 \mathrm{~nm}$ (mean $\pm \mathrm{SD}, 161$ mitochondria; $p<0.001 ; \mathrm{p}<$ 0.05 to cultivation in sodium-pyruvate). For determination of the $p$-values, see supplementary Figure 43.

Next, parameters of the inner mitochondrial membrane were manually determined in the same images used for diameter measurements using imageJ. Since complexes for the respiratory chain are located on the crista membranes (157), the abundance of these membranes was determined in relation to the mitochondrial area. This should give a rough estimation of the potential respiratory performance of these organelles. First, the area of each mitochondrion was measured. Then, the length of each crista within that mitochondrion was determined. Each data point represents the sum of all crista membrane lengths within one mitochondrion divided by the area of that mitochondrion (Figure 16).
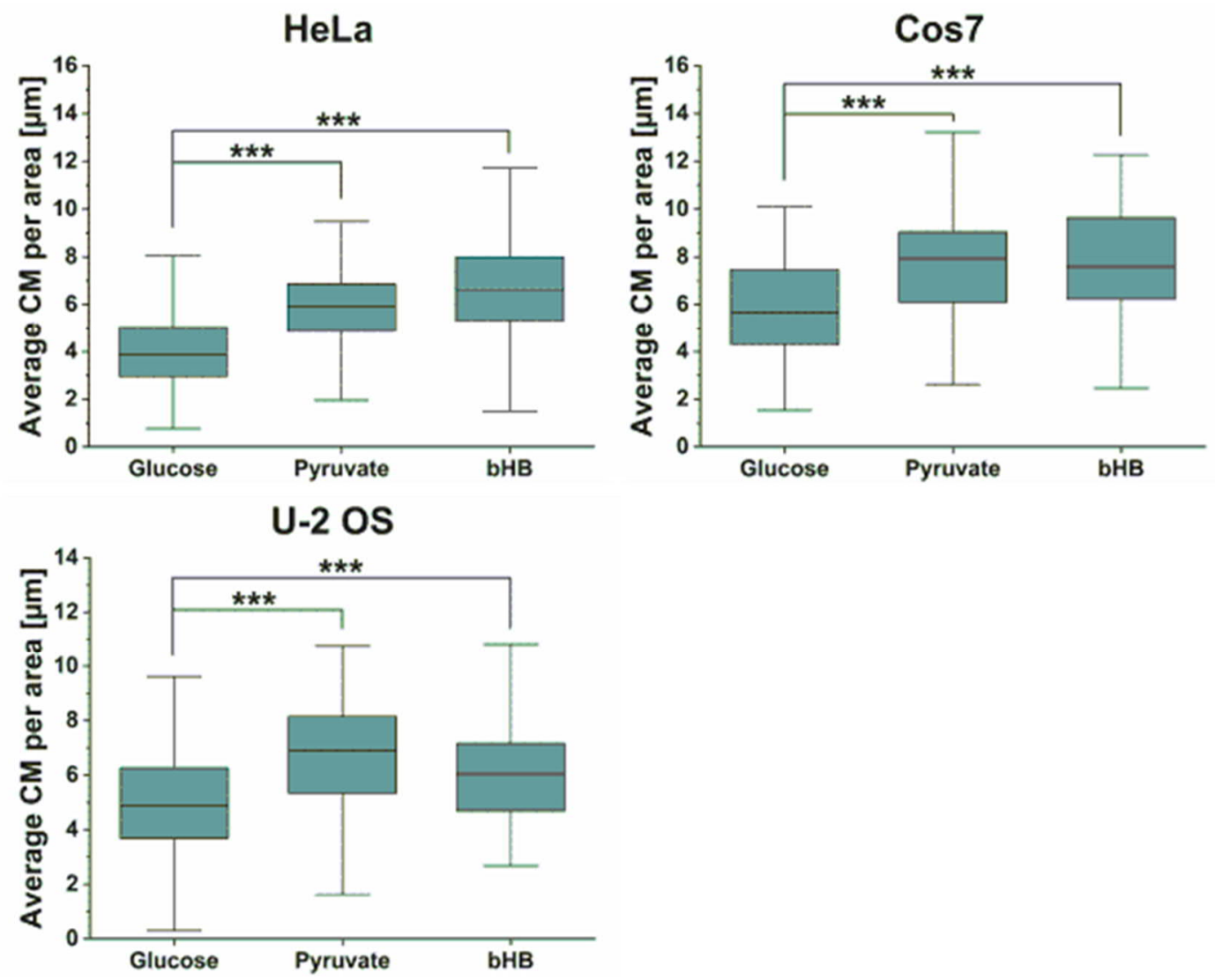

Figure 16: Average crista length per area of mitochondrial cross-section. The box plots show the abundance of crista membranes per area in $\mu \mathrm{m} / \mu m^{2}$ for HeLa, Cos7 and U-2 OS cells respectively. Box and whiskers plots show the mitochondria diameter obtained by manual measurements. The boxes indicate $25^{\text {th }}$ to $75^{\text {th }}$ percentile. The horizontal lines indicate median. Whiskers indicate $S D .{ }^{* * *} p<0.001$. 
Precisely, for HeLa cells grown in $30 \mathrm{mM}$ glucose, the average crista length per area was 4.03 $\pm 1.58 \mu \mathrm{m} / \mu \mathrm{m}^{2}$ (mean $\pm \mathrm{SD}, 236$ mitochondria). After cultivation in presence of $10 \mathrm{mM}$ sodiumpyruvate, the length increased to $5.81 \pm 1.63 \mu \mathrm{m} / \mu \mathrm{m}^{2}$ (mean $\pm S D, 245$ mitochondria; $p<0.05$ ). With cultivation in presence of $25 \mathrm{mM}$ beta-hydroxybutyrate, the length increased to $6.62 \pm$ $1.94 \mu \mathrm{m} / \mu \mathrm{m}^{2}$ (mean $\pm \mathrm{SD}, 162$ mitochondria; $\mathrm{p}<0.05 ; \mathrm{p}<0.05$ to cultivation in sodiumpyruvate). U-2 OS cells showed an average crista length per area of $5.07 \pm 2 \mu \mathrm{m} / \mu \mathrm{m}^{2}$ (mean $\pm \mathrm{SD}, 217$ mitochondria) when grown in presence of $30 \mathrm{mM}$ glucose. After cultivation in presence of $10 \mathrm{mM}$ sodium-pyruvate, the length increased to $6.80 \pm 2.02 \mu \mathrm{m} / \mu \mathrm{m}^{2}$ (mean $\pm \mathrm{SD}$, 103 mitochondria; $p<0.05)$. The length after cultivation in presence of $25 \mathrm{mM}$ betahydroxybutyrate was $6.08 \pm 1.75 \mu \mathrm{m} / \mu \mathrm{m}^{2}$ (mean $\pm S D, 137$ mitochondria; $p<0.05 ; p<0.05$ to cultivation in sodium-pyruvate).

Cos 7 cells cultivated in $30 \mathrm{mM}$ glucose had an average crista length per area of $5.80 \pm 2.02$ $\mu \mathrm{m} / \mu \mathrm{m}^{2}$ (mean $\pm \mathrm{SD}, 129$ mitochondria). After cultivation in presence of $10 \mathrm{mM}$ sodiumpyruvate, the length increased to $7.73 \pm 2.24 \mu \mathrm{m} / \mu \mathrm{m}^{2}$ (mean $\pm S D, 150$ mitochondria; $p<0.05$ ). After cultivation in presence of $25 \mathrm{mM}$ beta-hydroxybutyrate, the length increased to $7.81 \pm$ $2.07 \mu \mathrm{m} / \mu \mathrm{m}^{2}$ (187 mitochondria; $p<0.05 ; p=0.733$ to cultivation in sodium-pyruvate). For determination of the $p$-values, see supplementary Figure 44.

In all cell lines analysed, an increase of the abundance of crista-membranes was observed when energy substrates were present that could only be utilized by mitochondria (sodiumpyruvate or beta-hydroxybutyrate). This effect was the strongest for HeLa CCL cells but the same trend was observed for Cos7 cells and U-2 OS cells as well (Figure 17). Compared to the glucose cultivation condition, a 2 -fold increase $(2.05 \pm 0.17$, mean $\pm S D)$ in crista membranes was observed when HeLa cells were cultivated in presence of $10 \mathrm{mM}$ sodiumpyruvate. The abundance increased to about 2.4-fold $(2.43 \pm 0.16$, mean $\pm S D)$ when HeLa cells were cultivated in presence of $25 \mathrm{mM}$ beta-hydroxybutyrate.

For U-2 OS cells, this increase was about 1.6-fold (1.63 \pm 0.14 for sodium-pyruvate and 1.63 \pm 0.10 for beta-hydroxybutyrate, mean $\pm S D$ ) for both cultivation conditions with respect to the glucose cultivation condition. In Cos7 cells, an increase of about 2 -fold $(2.05 \pm 0.17$, mean \pm $\mathrm{SD})$ was found for cultivation in presence of sodium-pyruvate and about 2.2-fold $(2.21 \pm 0.16$, mean $\pm S D$ ) increase for beta-hydroxybutyrate respectively. For determination of the $p$-values, see supplementary Figure $\mathbf{4 5 .}$ 

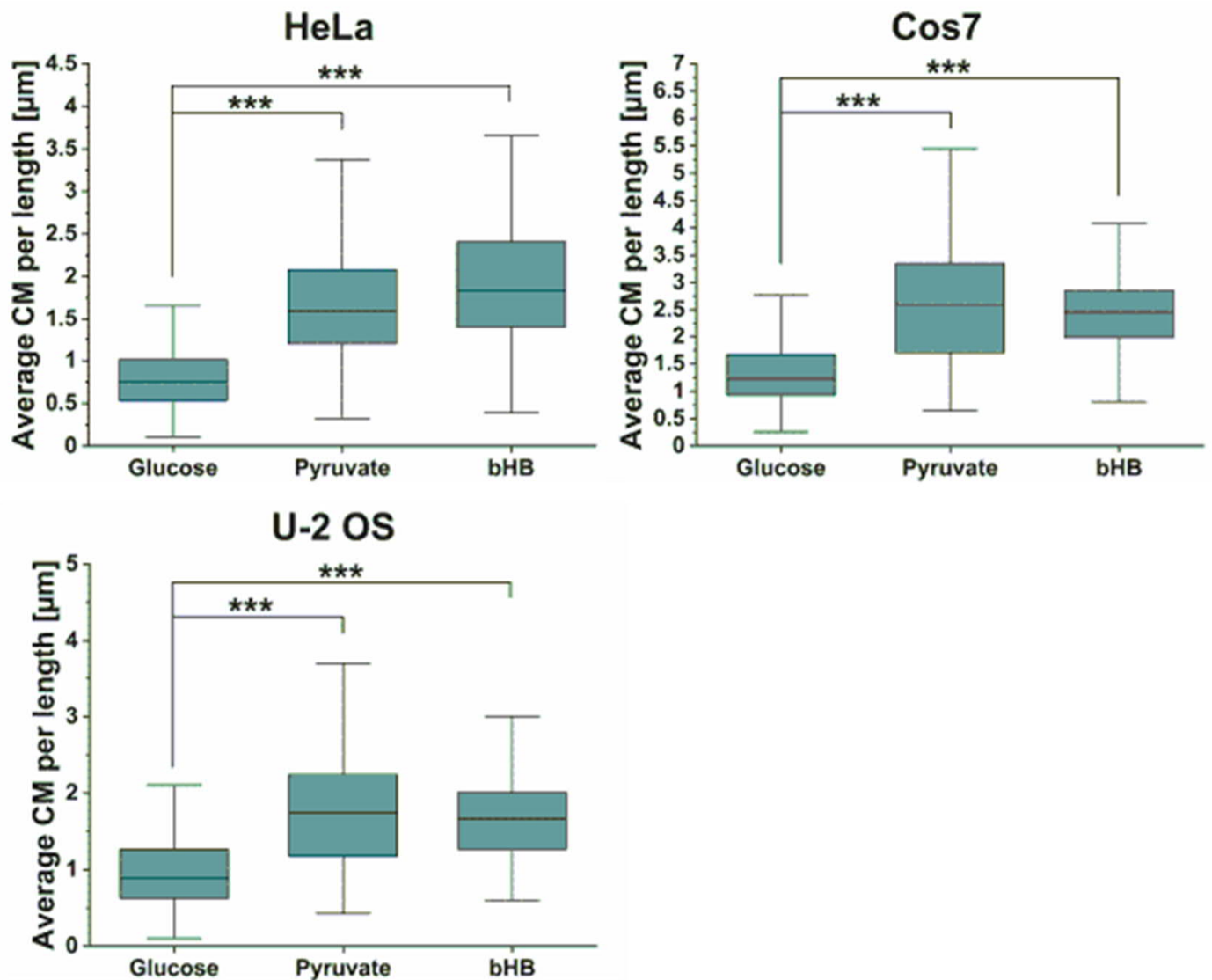

Figure 17: The Metabolic switch changes the length of crista membranes [ $\mu \mathrm{m}]$ per $\mu \mathrm{m}$ of mitochondrial length. Crista length in the mitochondria in cells cultivated in either $30 \mathrm{mM}$ glucose, $10 \mathrm{mM}$ sodium-pyruvate or $25 \mathrm{mM}$ beta-hydroxybutyrate normalized to cultivation condition in $30 \mathrm{mM}$ glucose. Analysed for HeLa, Cos7 and U-2 OS cells respectively. Box and whiskers plots show the mitochondria diameter obtained by manual measurements. The boxes indicate $25^{\text {th }}$ to $75^{\text {th }}$ percentile. The horizontal lines indicate median. Whiskers indicate $S D .{ }^{* * *} p$ $<0.001$.

In summary, the mitochondrial adaptation in HeLa, U-2 OS and Co7 cells was first tested in standard DMEM medium without serum supplementation. Either glucose, sodium-pyruvate or beta-hydroxybutyrate respectively was added to the medium. In this experimental setup, no adaptation of the mitochondrial ultrastructure could be observed. Instead, increased cell detachment and cell death was noticed in all cell lines.

The culture condition was then adapted using SILAC FLEX medium without glutamine that was further supplemented with dialyzed serum. There, significant changes in the mitochondrial architecture depending on the carbon source were shown. It was concluded, that indeed sodium-pyruvate and beta-hydroxybutyrate increased the respiratory activity of mitochondria while cells cultivated in presence of glucose relied primarily on glycolysis for the generation of ATP. The orientation of the crista membranes in parallel to the outer mitochondrial membrane indicated a lower performance of respiratory activity of mitochondria. 
The structural similarity of these mitochondria to presynaptic mitochondria in neuronal cells further indicates that presynaptic mitochondria might be as well, lower in respiratory performance. Taken together, a long-term structural adaptation of mitochondria could be induced with a metabolic switch in several cancer cell lines that resulted in a complex membrane remodelling. These preliminary experiments provided the basis to analyse the structural adaptations of mitochondrial in the neuronal presynapse.

\section{Mitochondrial occupation of rat hippocampal presynapses}

Mitochondria might locate to presynapses to supply ATP and support presynaptic function especially during long-lasting neuronal activity $(101,104,111)$. The high-energy demand during synaptic transmission would suggest that the majority of presynapses contain mitochondria. Further, the mitochondrial occupation of presynapses might increase during long stimulation of the neuronal cells.

In order to get a precise understanding of the distribution of mitochondria at the presynapses, rat hippocampal neurons were cultivated for 22 DiV on glass coverslips. A subset of the cultures was additionally depolarized using a final concentration of $25 \mathrm{mM} \mathrm{KCl}$ for one hour. The cells were then immobilized according to the CAF procedure using $2.5 \%$ glutaraldehyde in $0.1 \mathrm{M}$ cacodylic buffer ( $\mathrm{pH}$ 7.4). Staining and final resin embedding for electron microscopy followed the standard protocol.

Ultra-thin sections of $60 \mathrm{~nm}$ thickness were examined at $6500 x$ original magnification and areas of the sections containing cells automatically recorded with a 10x10 tile recording setting including a $20 \%$ overlap of neighbouring micrographs. This approach allows for an un-biased recording of cell areas and prevents the multiple imaging of the same synapses.

Electron microscopy of rat hippocampal neurons revealed that not all presynapses of nonstimulated neurons have mitochondria located at presynaptic sites (Figure 18 A, presynapses containing mitochondria are indicated with white arrows, presynapses without mitochondria are indicated with white stars).

Next, the mitochondrial occupation of presynapses was analysed in neuronal cultures that were chemically depolarized with $25 \mathrm{mM} \mathrm{KCl}$ for one hour prior to fixation (Figure $18 \mathrm{~B}$ ). Precisely, 1830 images were analysed for depolarized cultures containing 4301 synapses of which 1514 contained mitochondria (34.4 $\pm 0.68 \%$ ). In the control, 1760 images were acquired containing 2745 presynapses of which 928 contained mitochondria (35.8 $\pm 0.44 \%)$. 

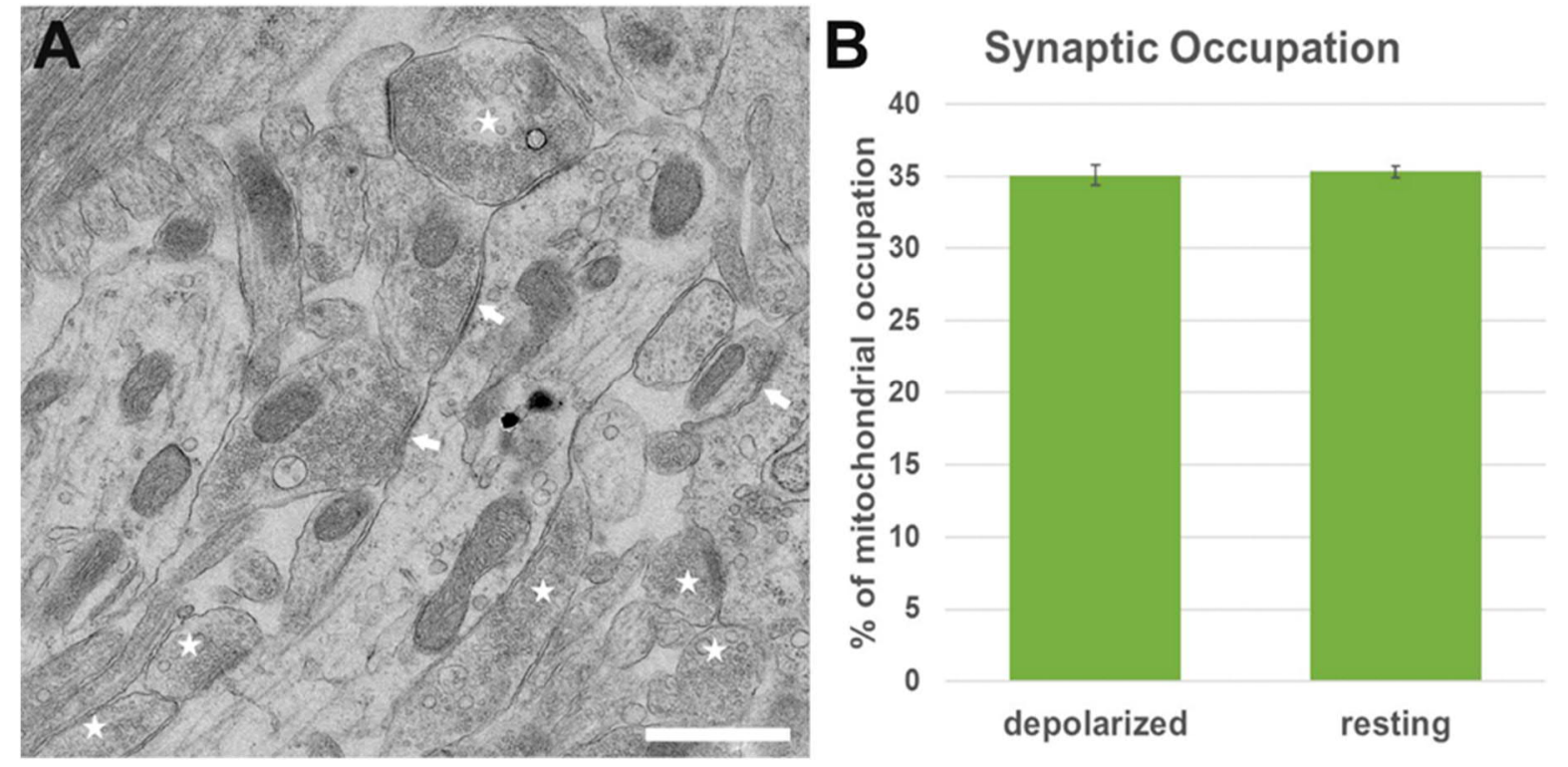

Figure 18: Mitochondrial occupation of presynapses in cultured rat hippocampal neurons. A: Electron micrograph showing multiple presynapses in a 2D culture of rat hippocampal neurons cultivated for 22 DiV. Presynapses containing mitochondria are marked with a white arrow. Presynapses without mitochondria are labelled with a white star. B: Evaluation of the mitochondrial occupation of neuronal presynapses after cultivation for 22 DiV. Mitochondrial occupation compared between control cultures and cultures with additional depolarization for one hour (25 $\mathrm{mM} \mathrm{KCl}$ ). Scale bar in $A=1 \mu \mathrm{m}$.

In cultures of rat hippocampal neurons without stimulation of synaptic activity, the majority of presynapses lack mitochondria. The mitochondrial occupation of presynapses was further not influenced by long-term chemical depolarization of the cells. This suggest, that the mitochondrial network of rat hippocampal neurons does not adapt to an increased energy demand at the presynapse by transporting more mitochondria to presynaptic boutons. A detailed analysis of presynaptic mitochondria is needed to assess their structure in resting neurons and the structural adaptation of the inner membrane architecture after long-term presynaptic activity. 


\section{Cultivation of rat hippocampal neurons in presence of different carbon substrates}

Under normal conditions, neuronal mitochondria display a variety of inner mitochondrial membrane morphologies (See Figure 9 and Figure 19 respectively) depending on their localization within the cell body. Neuronal mitochondria located in the soma or the dendritic cell extensions display a crista-membrane arrangement in a lamellar fashion.

Soma

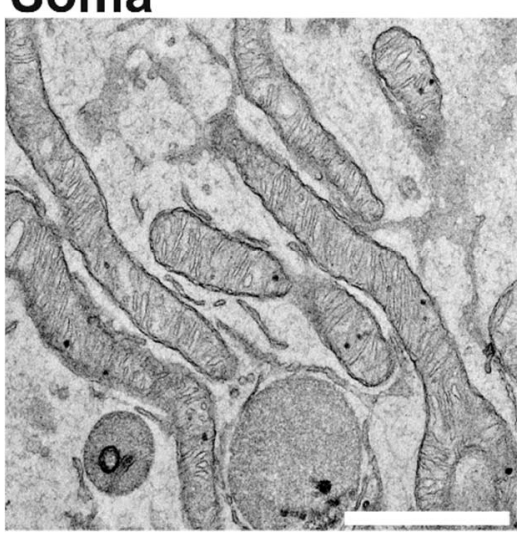

Neurites

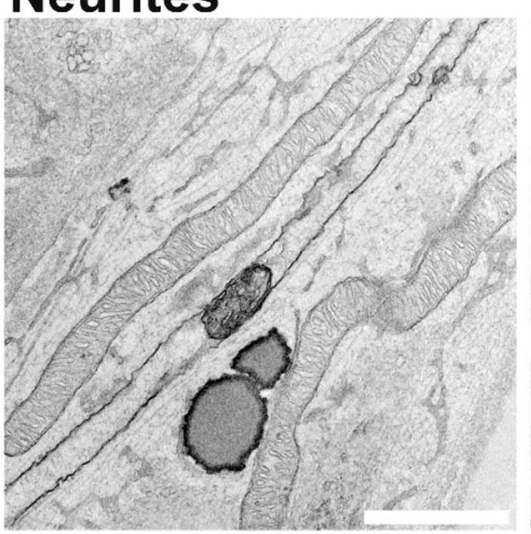

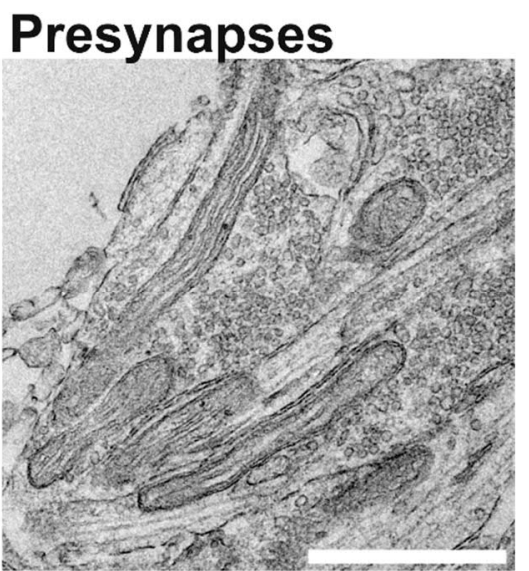

Figure 19: Transmission electron microscopy of the mitochondrial inner membrane architecture at different neuronal sites. Left: Mitochondria in the soma of rat hippocampal neurons. Mitochondria appear as elongated tubes with crista membranes perpendicular to the outer mitochondrial membrane. Middle: Mitochondria in neurites. Mitochondria are elongated and the crista membranes are perpendicular to the outer mitochondrial membrane. Right: mitochondria in neuronal presynapses. Mitochondria are short and display a smaller diameter as compared to other cellular sites of the neurons. Crista membranes are in parallel to the outer mitochondrial membrane. Scale bars in left and middle panel $=1 \mu \mathrm{m}$. Scale bar in right panel $=500 \mathrm{~nm}$.

This arrangement of inner mitochondrial membranes is found as well in mitochondria of cultivated cancer cells such as HeLa or Cos7 cells. The diameter, as obtained from mitochondria in these areas, is $400 \mathrm{~nm}$. However, in axons and synapses, a different arrangement of the crista membranes is present. In most synapses and in virtually every axonal mitochondrion, the crista membranes are oriented in parallel to the inner boundary membrane as well as the outer membrane. In addition, the size of the mitochondria in presynapses is reduced compared to mitochondria in the soma, showing a diameter of approximately $180 \mathrm{~nm}$.

The populations of mitochondria show two classes, on the one hand, somatodendritic mitochondria that are similar in appearance and shape as mitochondria seen in standard cultivation conditions of cancer cells as well as during cultivation in the presence of betahydroxybutyrate (Figure 20 A and B). On the other hand axonal and pre-synaptic mitochondria similar to mitochondria in cancer cells cultivated in glucose without the addition of glutamine (Figure $20 \mathrm{C}$ and D). 


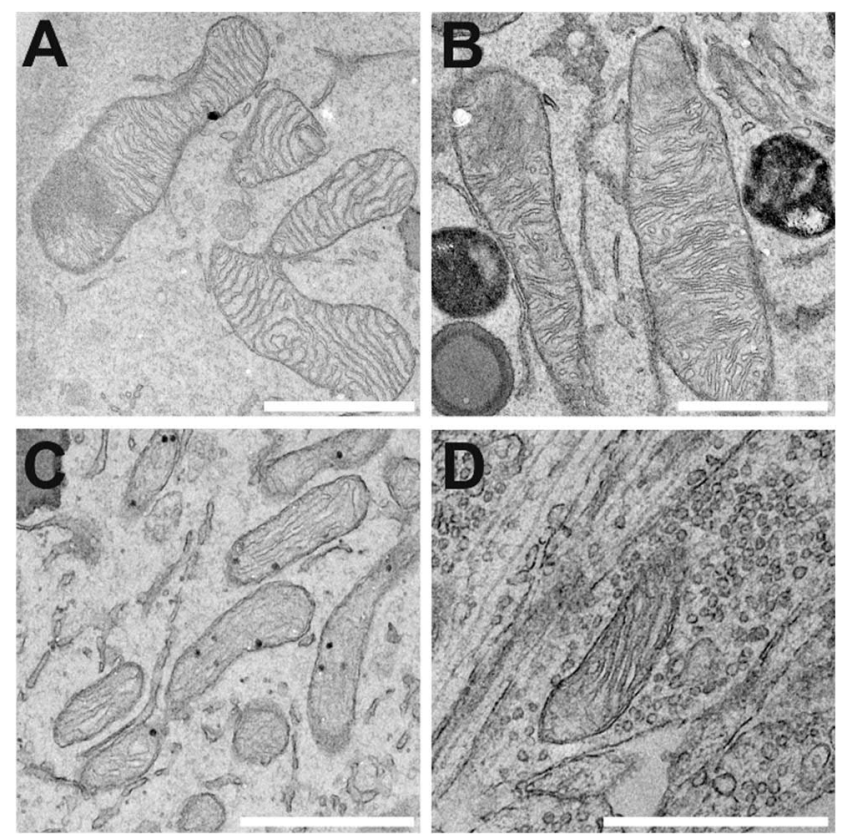

Figure 20: Comparison of mitochondria across model systems. Transmission electron micrographs of Cos7 cells and rat hippocampal neurons reveals structural similarities of mitochondria. A: Mitochondria in Cos7 cells cultivated in presence of $25 \mathrm{mM}$ betahydroxybutyrate. B: Mitochondria in the neuronal soma. C: Cos7 cells cultivated in the presence of $30 \mathrm{mM}$ glucose. D: Mitochondria in the neuronal presynapse. Scale bars $=1 \mu \mathrm{m}$.

To study the impact of different carbon sources on mitochondrial inner membrane architecture in neuronal cells, rat hippocampal neurons were isolated from P0 rats, seeded on $18 \mathrm{~mm}$ PLLcoated glass coverslips and initially cultivated according to the standard procedures. After 4 DiV, the proliferation of fibroblasts and glia-cells in the culture was reduced by the addition of $5 \mu \mathrm{M}$ ara-C into the medium. This time point was chosen as adding the supplement at an earlier time of the culture resulted in the death of most of the neuronal cells and adding the supplement later than $7 \mathrm{DiV}$ was insufficient in reducing the cell number of proliferating cells in the culture (data not shown).

After $6 \mathrm{DiV}, 40 \%$ of the culture medium was aspirated and replaced with Neurobasal-A medium, without glucose and without sodium-pyruvate but supplemented with PenStrep and $25 \mathrm{mM}$ beta-hydroxybutyrate. This medium exchange was carried out every other day up to $12 \mathrm{DiV}$ in order to replace any available glucose in the culture with ketone bodies. This also helped to reduce the concentration of ara- $C$ to minimize any adverse long-term effects on the neuronal cells. Cultivation of the neurons was continued for another 10 days in presence of beta-hydroxybutyrate up to 22 days in vitro. Control cultures of rat hippocampal neurons were treated in the same way by using standard cultivation medium with the supplementation of 
glucose. All cells were then immobilized using $2.5 \%$ glutaraldehyde in $0.1 \mathrm{M} \mathrm{SPB}$ at pH of 7.4. Staining and final resin embedding for electron microscopy followed the fBROPA protocol.

Soma

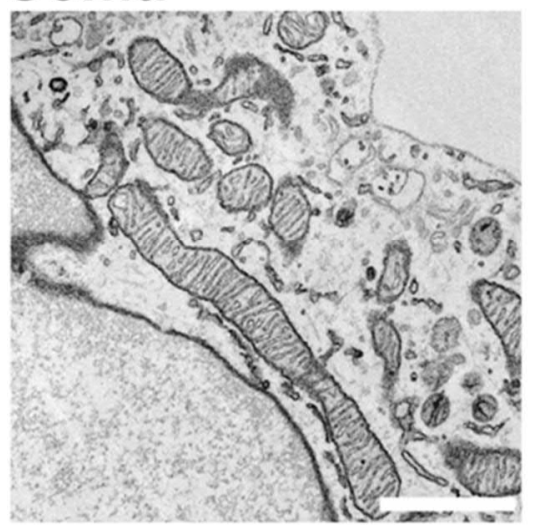

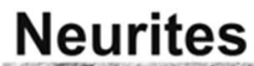

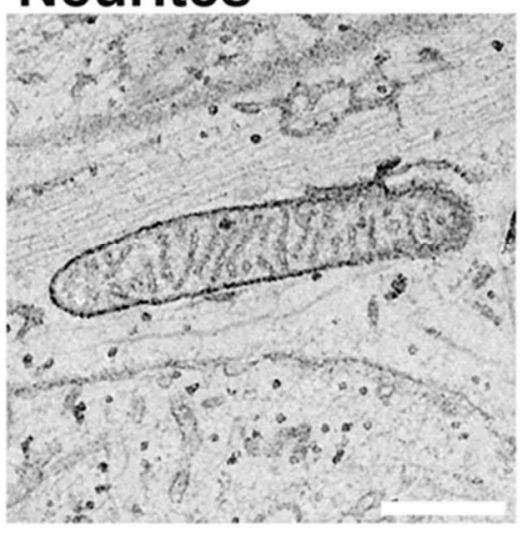

Presynapses

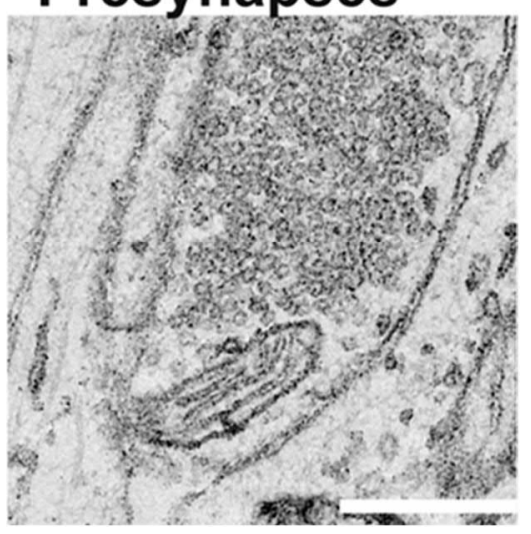

Figure 21: Exemplary transmission electron micrographs of mitochondria in hippocampal neurons grown in presence of $25 \mathrm{mM}$ beta-hydroxybutyrate. Left: Mitochondria found in the soma of neuronal cells. Scale bar $=1 \mu \mathrm{m}$. Middle: Mitochondria localized in the neurites. Scale bar $=500 \mathrm{~nm}$. Right: Mitochondria localized in the neuronal presynapse. Scale bar $=500 \mathrm{~nm}$.

Ultimately, ultra-thin sections of $60 \mathrm{~nm}$ thickness were prepared and placed on Formvar-coated slot grids. The grids were examined at $120 \mathrm{kV}$ with $6500 \mathrm{x}$ original magnification. Mitochondria from different sites of the neurons were imaged. The change in energy substrate availability did not result in signs of cellular stress and cell death of the neuronal cultures. Electron micrographs of somatic and dendritic mitochondria showed a stack-like arrangement of cristamembranes (Figure 21). Notably, the axonal and presynaptic mitochondria showed a parallel arrangement of the crista-membranes to the outer mitochondrial membrane.

The ultrastructure of presynaptic mitochondria is similar to mitochondria found in the control cultures. Hence, the cultivation of hippocampal neurons in presence of beta-hydroxybutyrate and the accompanying metabolic switch did not result in a change of the mitochondrial inner architecture at presynaptic sites. The dependency of the membrane architecture on mitochondrial activity might indicate, that the basal synaptic activity of the neurons is low and therefore, mitochondria do not need to adapt. Stimulated synaptic activity could further increase the energy demand at the presynapses in neurons grown in presence of betahydroxybutyrate and thus, might cause a structural adaptation of the mitochondria. To this end, rat hippocampal neurons needed to be stimulated by chemical depolarization. 


\section{Mitochondrial inner membrane architecture after chemical depolarization}

Rat hippocampal neurons did not show a structural rearrangement to the change in energy substrate present in the cultivation medium. The inner membrane architecture of neuronal mitochondria was further investigated during chemical depolarization with high concentrations of potassium chloride $(\mathrm{KCl})$.

To this end, rat hippocampal neurons were isolated from P0 rats, seeded on $18 \mathrm{~mm}$ PLLcoated glass coverslips and initially cultivated according to standard procedures. After $4 \mathrm{DiV}$, the proliferation of fibroblasts and glia-cells in the culture was prohibited by the addition of ara$\mathrm{C}$ into the medium with a final concentration of $5 \mu \mathrm{M}$. After $6 \mathrm{DiV}, 40 \%$ of the culture medium was aspirated and replaced with Neurobasal-A medium, no glucose, no sodium-pyruvate, which was further supplemented with PenStrep and $25 \mathrm{mM}$ beta-hydroxybutyrate. This medium exchange was carried out every other day up to $12 \mathrm{DiV}$ in order to replace the available glucose in the culture with ketone bodies. After $12 \mathrm{DiV}$, the cultivation of the neurons continued for another 10 days in presence of beta-hydroxybutyrate. Control cultures of rat hippocampal neurons were treated accordingly by using standard cultivation medium with containing $30 \mathrm{mM}$ glucose. After 22 DiV, both culturing conditions were then depolarized with a final concentration of $25 \mathrm{mM} \mathrm{KCl}$ for 1 hour and for 4 hours respectively. All cells were then immobilized by chemical fixation using $2.5 \%$ glutaraldehyde in $0.1 \mathrm{M} \mathrm{SPB}$ at $\mathrm{pH}$ of 7.4.
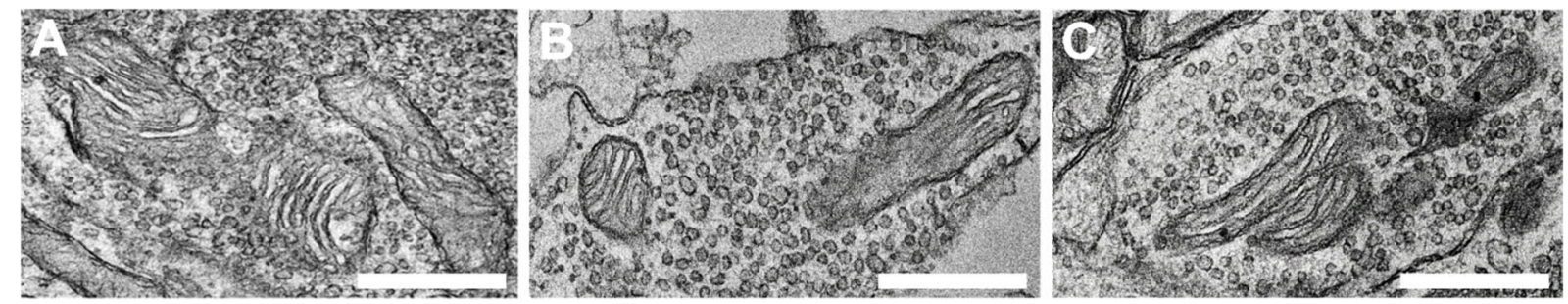

Figure 22: Exemplary transmission electron micrographs of presynaptic mitochondria of neurons after cultivation for 21 DiV. A: Presynaptic mitochondria of neurons cultivated in presence of $30 \mathrm{mM}$ glucose. B: Presynaptic mitochondria of neurons cultivated in presence of $25 \mathrm{mM}$ beta-hydroxybutyrate. C: Presynaptic mitochondria of neurons cultivated in presence of $25 \mathrm{mM}$ beta-hydroxybutyrate with 4 hours of depolarization $(25 \mathrm{mM} \mathrm{KCl})$ prior to chemical immobilization. Scale bars $=200 \mathrm{~nm}$.

Staining and final resin embedding for electron microscopy followed the adapted high-contrast protocol. Ultimately, ultra-thin sections of $60 \mathrm{~nm}$ thickness were prepared and placed on Formvar-coated slot grids. The grids were then examined at $120 \mathrm{kV}$ with $8600 \mathrm{x}$ original 
magnification. Specifically, presynaptic sites containing mitochondria were imaged (Figure 22).
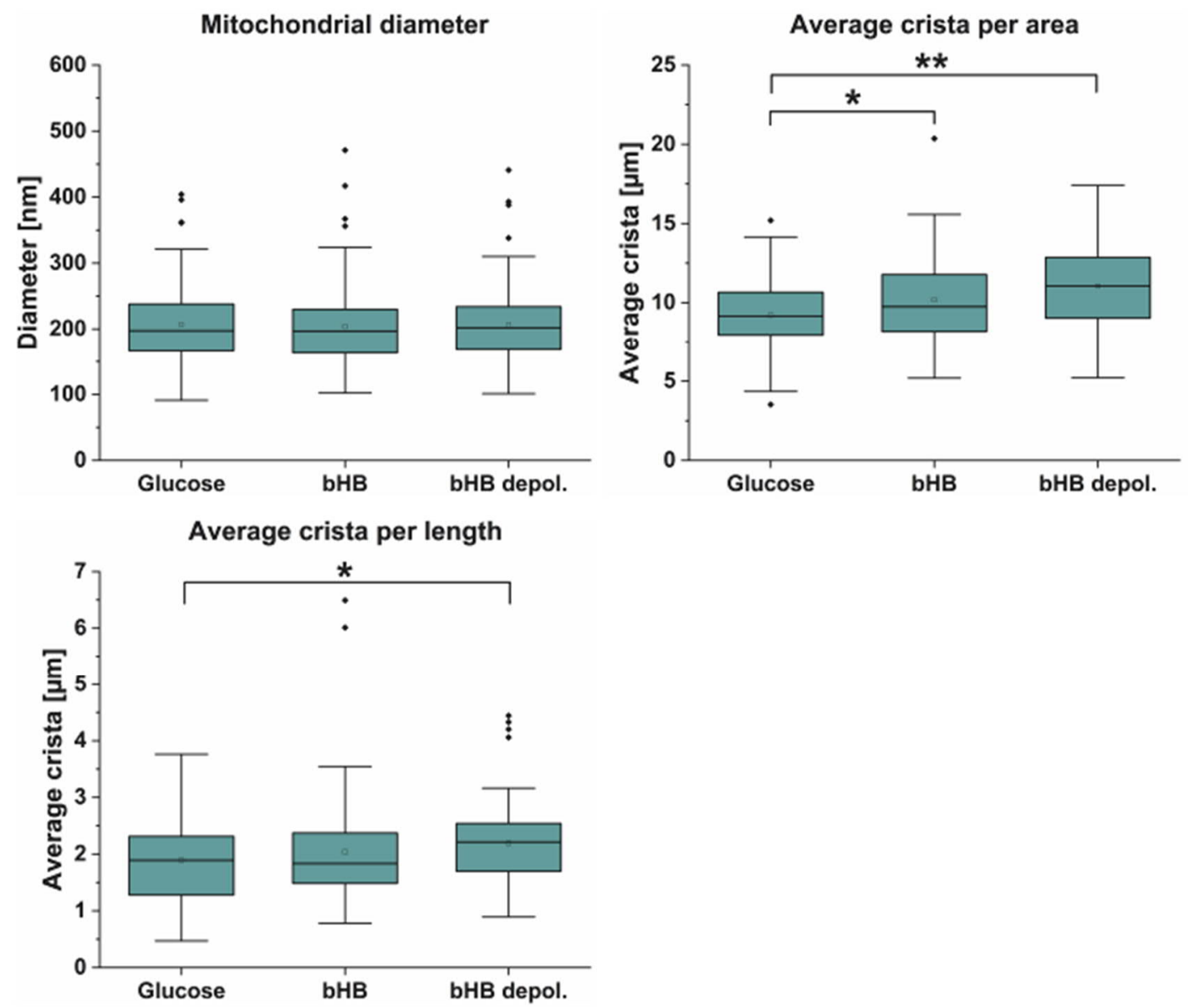

Figure 23: Morphological measurements in presynaptic mitochondria of rat hippocampal neurons after 21 DiV. Cells were cultivated in $30 \mathrm{mM}$ glucose (Glucose) or $25 \mathrm{~mm}$ betahydroxybutyrate (bHB) and grown in $25 \mathrm{mM}$ beta-hydroxybutyrate with additional chemical depolarization for four hours ( $\mathrm{bHB}$ depolarized with $25 \mathrm{mM} \mathrm{KCl}$ ). The mitochondrial diameter and area as well as crista membrane length were measured manually in $2 D$ transmission electron micrographs. Left: Box plots of the diameter of presynaptic mitochondria in nanometres. Right: Average crista length per area of mitochondrial cross section in $\mu m$ per $\mu \mathrm{m}^{2}$. Lower left: Box plots of the average crista abundance per mitochondrial length in $\mu \mathrm{m}$. The boxes indicate $25^{\text {th }}$ to $75^{\text {th }}$ percentile. The horizontal lines indicate median. The small centre box indicates the mean. Whiskers indicate $S D .{ }^{*} p<0.05 .{ }^{* *} p<0.01$.

These images were then used to determine first the mitochondrial diameter. Subsequently, the abundance of crista membranes in relation to the area of the mitochondrial cross section was determined. All measurements were performed using imageJ (Figure 23). The mitochondrial diameter for neurons cultivated in presence of $30 \mathrm{mM}$ glucose was $207.01 \pm 60.89 \mathrm{~nm}$ (mean \pm SD, 92 mitochondria). For neurons cultivated in presence of $25 \mathrm{mM}$ beta-hydroxybutyrate a diameter of $203.70 \pm 57.28 \mathrm{~nm}$ (mean $\pm S D, 155$ mitochondria; $p=0.67$ ) was obtained. For neurons cultivated in presence of $25 \mathrm{mM}$ beta-hydroxybutyrate with additional depolarization 
for four hours, a diameter of $206.14 \pm 52.64 \mathrm{~nm}$ (mean \pm SD, 177 mitochondria; $p=0.90$; to control condition in bHB $p=0.69$ ) was obtained (Figure 23).

Measurements of the average crista length per area of mitochondrial cross section resulted in $9.22 \pm 2.32 \mu \mathrm{m} / \mu \mathrm{m}^{2}$ (mean $\pm \mathrm{SD}, 68$ mitochondria) for neurons cultivated under standard conditions. This value showed a slight increase for both of the neurons cultivated in presence of beta-hydroxybutyrate to $10.19 \pm 2.66 \mu \mathrm{m} / \mu^{2}{ }^{2}$ (mean $\pm S D, 67$ mitochondria; $p<0.05$ ). For neurons cultivated in presence of beta-hydroxybutyrate with additional depolarization for four hours this resulted in $11.06 \pm 2.61 \mu \mathrm{m} / \mu \mathrm{m}^{2}$ (mean $\pm \mathrm{SD}, 70$ mitochondria; $p<0.05$; to control condition in bHB $\mathrm{p}=0.059$ ) (Figure 23).

Last, the average crista length per length of the mitochondria was evaluated. For neurons cultivated in presence of $30 \mathrm{mM}$ glucose, the average crista membrane length was $1.89 \pm 0.78$ $\mu \mathrm{m}$ per $\mu \mathrm{m}$ mitochondrial length (mean $\pm \mathrm{SD}$ ). When cultivating neurons in presence of $25 \mathrm{mM}$ beta-hydroxybutyrate, the average crista membrane length increased to $2.03 \pm 0.94 \mu \mathrm{m}$ per $\mu \mathrm{m}$ of mitochondria (mean $\pm S D, p=0.35$ ). Cultivation of neuronal cells in presence of $25 \mathrm{mM}$ beta-hydroxybutyrate with additional depolarization $(25 \mathrm{mM} \mathrm{KCl})$ for four hours resulted in an average crista membrane length of $2.19 \pm 0.74 \mu \mathrm{m}$ per $\mu \mathrm{m}$ of mitochondria (mean $\pm \mathrm{SD}, \mathrm{p}=$ $0.025 ; p=0.28$ to cultivation in presence of $25 \mathrm{mM}$ beta-hydroxybutyrate with no chemical depolarization) (Figure 23). For determinations of the p-values, see supplementary Figure 46.

These results demonstrated that, unlike in the preliminary experiments with cancer cells, the morphology of presynaptic mitochondria in neurons is not significantly influenced by the carbon source available for the cell metabolism. However, the abundance of crista membranes in neurons grown in presence of beta-hydroxybutyrate with additional chemical depolarization significantly increased. Interestingly, this increase was not associated with a structural remodelling of the membranes.

Cultures of rat hippocampal neurons are a well-established in-vitro system to study mechanistic aspects of presynaptic function. The membrane architecture of presynaptic mitochondria in these cells could allow for the supply of ATP required for synaptic transmission. However, the activity of presynapses in in-vitro cultures might be lower compared to brain tissue since the neurons lack cellular interplay with other cells supporting synaptic function such as glia-cells and astrocytes. Presynapses of neurons in the auditory complex are a model system for high-fidelity synapses (111). These neurons cannot be cultured and therefore need to be imaged in brain preparations. Structural details of mitochondria in these presynapses could be analysed in-vivo and compared to mitochondria in presynapses of rat hippocampal neurons in-vitro. 


\section{In-vivo analysis of presynaptic mitochondria}

The auditory complex poses a high-throughput network of neuronal cells. Because of the high activity of synaptic transmission in this brain area, presynapses like the calyx of Held were used as a model system for high-fidelity synapses. The calyx of Held is a highly specialized synapse and a comparison to smaller synapses might be less straightforward. Smaller synapses in the same brain area however, could be used to study the mitochondrial architecture in minor boutons and compared to in-vitro cultures of hippocampal neurons. Neurons derived from the auditory complex cannot be cultivated in-vitro. This eliminates the possibility to let the cells mature in culture and to additionally apply protocols for neuronal stimulation. The auditory complex of rodents develops from day 12 after birth (P12) with an increasing specialisation of this brain area after onset of the ability to hear. The onset of hearing marks a point where neurons transition from mostly infrequent and random synaptic activity to a highly streamlined activity of the synapses in order to process sound information.

This model system was used to study the overall distribution of mitochondria in the neuronal presynapse before and after the animals gaining the ability to hear. Precisely, the cochlear nucleus of animals at the age of nine days (P9) as well as in the age of 20 days (P20) was imaged using 3D FIB-SEM. The dissection, staining and embedding of the tissue slices for electron microscopy was prepared and provided by Anika Hinze in the laboratory of Prof. Dr. Carolin Wichmann. Dr. Anna Steyer in the laboratory of Dr. Wiebke Möbius carried out the acquisition of the FIB-SEM micrographs.

In three different sets of electron micrographs (volumes) of both conditions, presynapses were identified based on characteristic features such as abundance of a synaptic vesicle pool (SVs), abundance of an active zone as well as the abundance of a postsynaptic density (PSD). For each animal (P9 or P20), at least two independent data stacks were analysed.

First, all datasets were analysed considering presynapses present within the field of view. All synapses were separated in two classes namely presynapses without mitochondria and presynapses with mitochondria. In the P9 animal, 222 presynapses were identified in the electron micrographs with $~ 55 \%$ of them showing an occupation with mitochondria (55.28 \pm $1.62 \%$ ). For the P20 animal, 162 presynapses were identified in the micrographs with $80 \%$ of them showing an occupation with mitochondria $(79.95 \pm 1.49 \%$, mean $\pm S D, P<0.05$, (Figure 24 A).

Next, a random subset of the presynapses as previously identified in each data stack was chosen for a more detailed structural analysis. Precisely, the presynaptic bouton, the synaptic vesicle pool, the PSD as well as the mitochondria were manually segmented in 15 presynapses for each sub-pool (P9 with and without mitochondria, P20 with and without mitochondria) using 
IMOD (Figure 24 B)(158). In the following, the resulting models were analysed in Amira 6.04 (ThermoFisher Scientific, Waltham, MA, USA) resulting in volume measurements of the bouton volume, the PSD volume and the mitochondrial volume respectively. All object meshes were imported from the IMOD model without any subjection to surface smoothing (Figure $24 \mathrm{C}$ ).
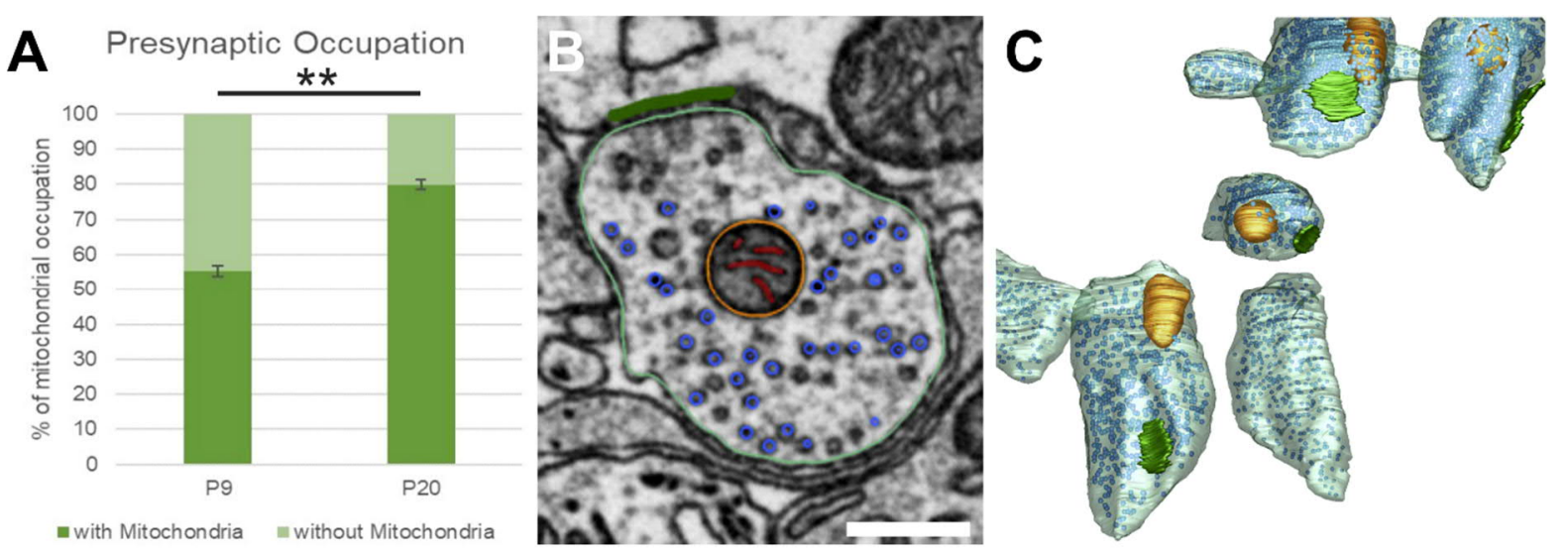

Figure 24: Structural analysis of neuronal presynapses in-vivo. 3D FIB-SEM acquisition revealed multiple minor synapses in the cochlear nucleus of $P 9$ and $P 20$ animals. All synapses were analysed for the occupation with mitochondria. Subsequently, structural details were analysed in presynapses with and without mitochondria for both ages. A: Quantification of the mitochondrial occupation of presynapses in the cochlear nucleus of a P9 and a P20 animal respectively. 222 synapses identified for the P9 animal (3 FIB-SEM volumes). 162 synapses identified in the $P 20$ animal (3 FIB-SEM volumes). B: Exemplary scanning electron micrograph of the $P 9$ animal used for segmentation, light green: presynaptic bouton, orange: mitochondrion, green: postsynaptic density, blue: synaptic vesicles, red: crista membranes. C: Exemplary 3D model showing various presynapses with and without mitochondria in the P9 animal. Scale bar $=300 \mathrm{~nm} .{ }^{* *} \mathrm{p}<0.01$.

Synaptic vesicles were manually picked in IMOD and marked as a single contour sphere with the size of six pixels on the micrograph at the centre of the vesicle. Synaptic vesicle numbers were obtained by direct readout of the number of contours in IMOD. Values for the volume of each feature were calculated in voxels with the size of a voxel of $125 \mathrm{~nm}^{3}(5 \mathrm{~nm} \times 5 \mathrm{~nm} \times 5 \mathrm{~nm}$ isotropic pixel size).

The bouton volume, the size of the synaptic vesicle pool as well as the size of the PSD were increased in presynapses with a mitochondrion being present in the bouton (Figure 25). Furthermore, an overall increase of these values was observed in the older animal after onset of hearing.

The average bouton size with mitochondrial occupation in P9 animals was about 4.7-fold increased $(p=0.0023)$. The average bouton size with mitochondrial occupation in P20 animals was about 2.4-fold increased ( $p=0.022)$. The increase of the average bouton size from P9 to 
P20 without the occupation with mitochondria was about 4-fold $(p=0.018)$ and from P9 to P20 with occupation of mitochondria about 2.-fold $(p=0.041)$.

\section{Bouton volume}

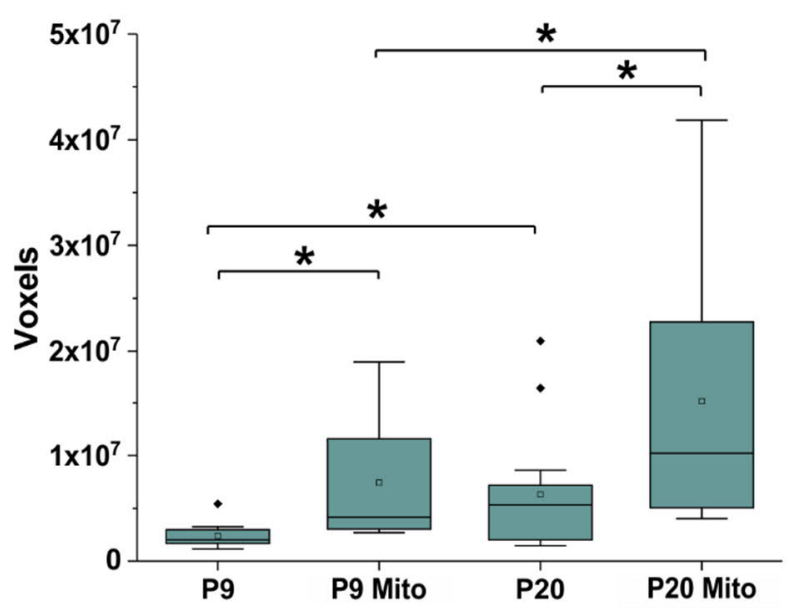

Synaptic vesicles

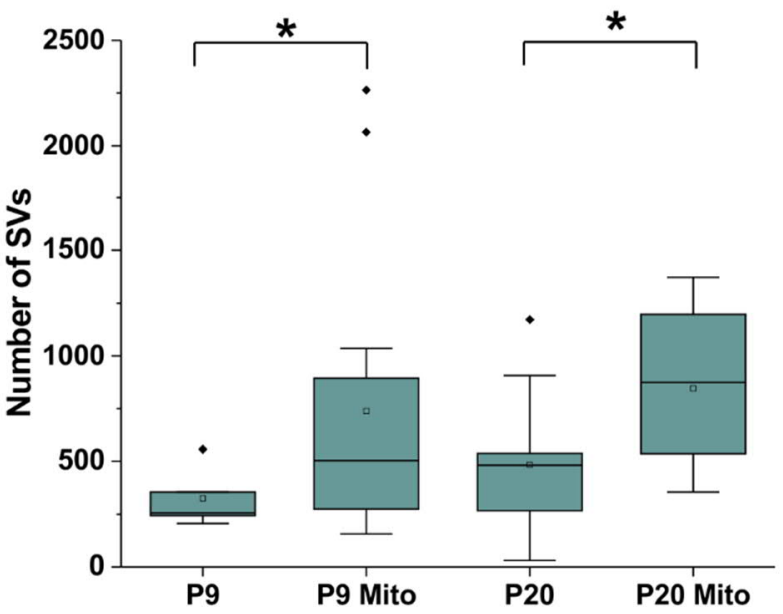

PSD volume

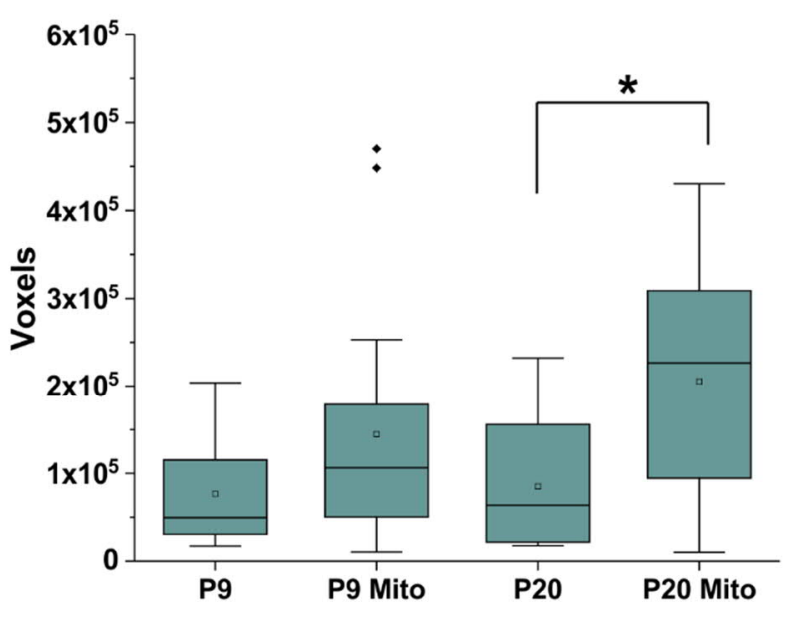

Mitochondrial volume

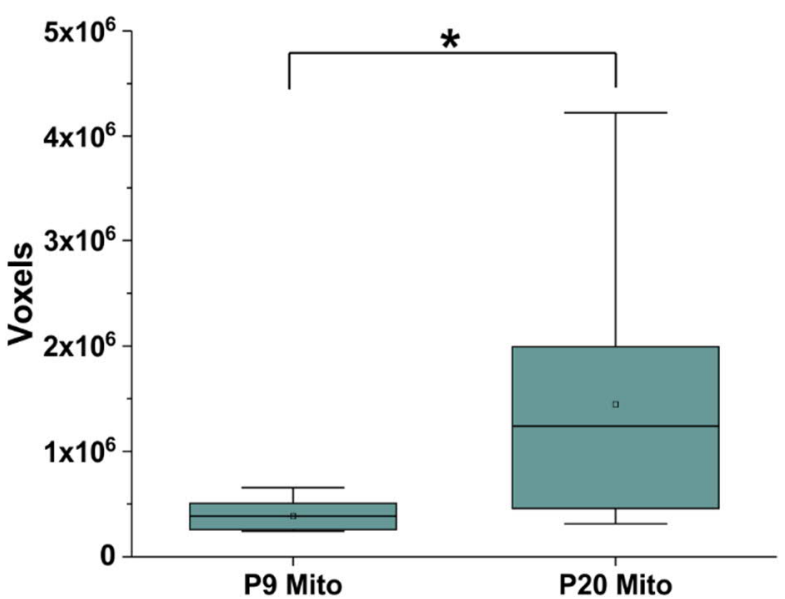

Figure 25: Quantification of the structural details of presynapses identified in a P9 animal as well as in a P20 animal. The box plots on the left show the distribution of the voxels obtained by volume measurements in Amira of the presynaptic boutons as well as the PSD volumes of all four conditions for 15 presynaptic boutons each. Box plots on the right display the abundance of synaptic vesicles obtained by counting the contours in IMOD of all four conditions in 15 presynaptic boutons each as well as the volume of presynaptic mitochondria of the P9 and the P20 animals obtained from measurements in Amira. The boxes indicate $25^{\text {th }}$ to $75^{\text {th }}$ percentile. The small centre box indicates the mean. The horizontal lines indicate median. Whiskers indicate $S D .{ }^{*} p<0.05$.

The average synaptic vesicle number in boutons with mitochondrial occupation in P9 animals was about 3.4-fold increased $(p=0.026)$. The average synaptic vesicle number in boutons with mitochondrial occupation in P20 animals was about 1.8 -fold increased $(p=0.005)$. The increase of the average synaptic vesicle number from P9 to P20 without the occupation with mitochondria was about 2.2-fold $(p=0.086)$ and from P9 to P20 with occupation of mitochondria about 1.2-fold ( $p=0.57)$. 
The average post-synaptic density size with mitochondrial occupation in P9 animals was about 2.9 -fold increased $(p=0.115)$. The average post-synaptic density size with mitochondrial occupation in P20 animals was about 2.4-fold increased $(p=0.0053)$. The increase of the average post-synaptic density size from P9 to P20 without the occupation with mitochondria was about 1.7-fold $(p=0.75)$ and from P9 to P20 with occupation of mitochondria about 1.4fold $(p=0.24)$.

The mitochondrial volume in the P20 animal increased about 5-fold $(p=0.011)$ compared to the P9 animal. The ratios of the synaptic vesicle abundance to the bouton size (SVs/Bouton Volume) as well to the PSD size (SVs/PSD Volume) were equal across the presynaptic subpopulations. In addition, ratios for the mitochondrial volume to the bouton size (Mitochondrial Volume/Bouton Volume) were increased in the P20 animal compared to the P9 animal (supplementary Figure 47).

In summary, the quantification of structures obtained by volume FIB-SEM imaging of the cochlear nucleus resulted in the quantification of structural details of presynapses and mitochondrial adaptation during the development of this brain area in mice. First, the higher abundance of mitochondria at presynaptic sites at P20 compared to P9 was an adaptation of the mitochondrial network in neuronal cells that was not observed in $2 \mathrm{D}$ cultures of rat hippocampal neurons. The increase of the abundance of synaptic vesicles, accompanied with an increase of PSD size suggests, that presynapses were more active in animals at P20 compared to P9. Strikingly, the mitochondrial volume in presynapses increased significantly during the development. This further indicated that indeed synapses in the auditory complex were more active compared to cultivated rat hippocampal neurons that resulted in a higher abundance of larger mitochondria at presynapses with high-energy demand in the cochlear nucleus. In the future, FIB-SEM imaging could be used on other brain areas than the auditory complex and the findings compared. This could include imaging of the hippocampus of brain preparations to compare both types of presynapses in-vivo at different developmental stages of the animals.

A mitochondrial adaptation at the neuronal presynapse was demonstrated in both, cultivated rat hippocampal neurons and neurons in the auditory complex. Especially 2D cell cultures of neurons offer the possibility to apply protocols of light microscopy and electron microscopy at high performance parameters. The protocols for cultivation and chemical depolarization presented in this study could be used for correlative imaging of cells. Correlative imaging would allow further investigating functional and structural relationships of presynaptic mitochondria in in resting and stimulated cultures of hippocampal neurons. To this end, protocols for the correlation of multiple imaging modalities needs to be established. 


\section{Section 2 - Protocol establishments for correlative imaging of cells}

In section 1, the optimisation of staining procedures for mitochondria and electron microscopy based investigations of the mitochondrial adaptation in cultured cancer cells and cultivated rat hippocampal neurons was presented. The data obtained in in-vitro systems was then compared to presynaptic mitochondria in-vivo in the cochlear nucleus of mice. Electron microscopy offers superior resolution to investigate cellular structures but due to the necessary sample fixation and preparation steps, it does not allow for live cell observations. Further, specific protein labelling strategies are challenging and require extensive optimization. An approach to address these shortcomings is the correlation of light and electron microscopy. In the second part of the results section, the development of methods for correlative imaging is described.

\section{Design of cell substrates for correlative imaging}

Successful correlative light and electron microscopy (CLEM) relies on the re-identification of the cells and features analysed in the light microscope using the electron microscope. A navigation tool and characteristic landmarks are needed to identify the area of interest in fluorescence microscopy as well as in electron microscopy. Therefore, the landmarks must be visible in both imaging modalities to allow registration and correlation of the datasets.

As an alternative to expensive commercially available CLEM substrates, I implemented a very robust and cheap strategy by using TEM finder grids to create a replica via carbon evaporation on the desired substrate. There, commercial TEM finder grids (e.g. London Finder Grids; Science Services) were placed on sheets of Aclar foil. A subsequent deposition of $10 \mathrm{~nm}$ carbon resulted in a pattern of sputtered carbon (sputter coater Bal-Tec Med 020) that can be easily visualized with any light microscope in brightfield or phase-contrast. Cells grown on these substrates and additionally stained with Mitotracker Deep Red FM were then imaged and recorded with their specific location on the substrate (Figure $26 \mathbf{A}-\mathbf{C}$ ).
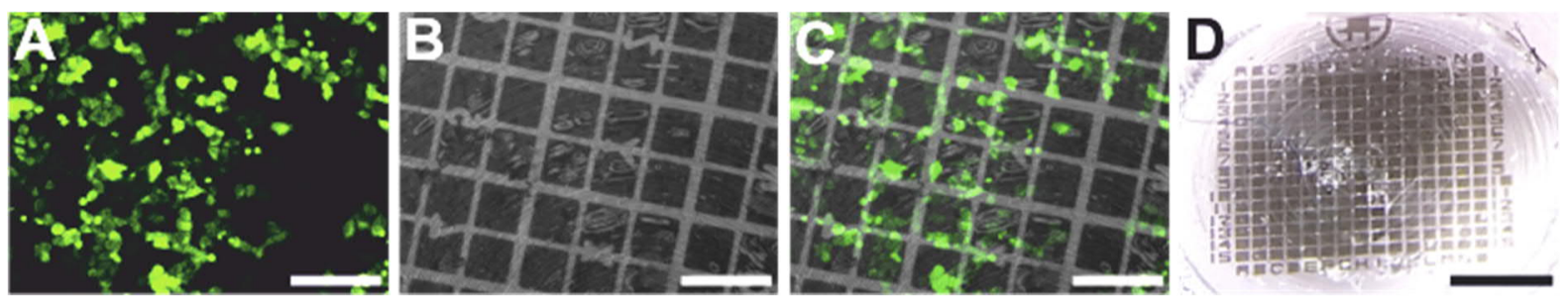

Figure 26: Development of substrates suitable for correlative imaging. A: Live-cell image of cells grown on a CLEM substrate, additionally labelled with Mitotracker Deep Red FM (green).

B: Brightfield image of the CLEM substrate revealing the finder-pattern. C: Overlay of $A$ and

$B$. D: The carbon-replica of the finder-grids transferred to the final resin blocks. Scale bars in $A, B$ and $C=200 \mu m$. Scale bar in $D=500 \mu m$. 
Most importantly, the carbon replica was completely transferred to the final resin blocks once the cells were immobilized and embedded in resin (Figure 26 D).

This index grid allowed an easy re-assignment of the cells in both light microscopy and embedded samples for electron microscopy. Alternatively, metal planchettes can be sputtered for cell immobilization by means of high pressure freezing with subsequent freeze substitution. With both substrates (Aclar or metal planchettes), cells could be first seeded and then treated in the desired fashion.

Taken together, carbon replicas of TEM grids created landmarks that were suitable to identify cells of interest first for light microscopy and subsequently for electron microscopy. The regions of interest first imaged with light microscopy could then be re-identified and the block face prepared for ultra-thin sectioning and transmission electron microscopy. This protocol allowed producing high numbers of substrates at a low cost to facilitate correlative imaging of live and fixed cells in light and electron microscopy.

\section{Live cell CLEM of Bax-EGFP expressing cells}

To establish protocols for correlative light and electron microscopy, I used a model system of intrinsic apoptosis of cells. Intrinsic apoptosis is mediated by mitochondria (159). Following an apoptotic stimulus, the pro-apoptotic protein Bax located in the cytosol is activated which leads to oligomerization and re-localization of Bax in the mitochondrial outer membrane $(160,161)$. Assemblies of activated Bax on mitochondria result in ring-like arrangements. This results in areas of the mitochondrial membrane that are devoid of highly abundant MOM proteins like Tomm20 (162). Ultimately, Bax localization on mitochondria is followed by efflux of downstream apoptotic factors such as cytochrome-C and progression of apoptosis. Activation of Bax can be achieved with transient overexpression of Bax for more than four hours. A fusionprotein with EGFP can be overexpressed, thus allowing live cell observations of Bax clustering on the mitochondria.

Early investigation of the mitochondrial architecture in apoptotic cells revealed mitochondrial outer membrane ruptures on a subset of mitochondria in these cells (163). However, correlative imaging is needed to analyse whether Bax oligomers co-localize with the mitochondrial membrane ruptures to correlate the function of Bax clusters to the membrane morphology at those sites.

To this end, HeLa cells were seeded on ACLAR polymer film that was previously carbon sputtered with a TEM grid replica for CLEM. At a confluency of approximately $70 \%$ the cells were transfected using $4 \mu \mathrm{g}$ of Bax-EGFP plasmid mixed with $6.67 \mu \mathrm{L}$ of Turbofect transfection 
reagent. Two hours after transfection the culture medium was replaced with fresh DMEM complete medium supplemented with $20 \mu \mathrm{M}$ Q-Vad-FMK. 30 minutes before fluorescence imaging, a final concentration of $200 \mathrm{nM}$ Mitotracker Deep Red FM was added to each well of the cells. This helped to visualize the shape and position of each cell within the population for subsequent re-identification in electron microscopy.

6 hours after transfection, the cell substrates were placed on a microscope slide and covered with a few drops of pre-warmed PBS ( $\mathrm{pH}$ at 7.4), in order to minimize the amount of phenol red in the supernatant during light microscopy imaging. The samples were then covered with a large glass coverslip to reduce the amount of liquid evaporation during fluorescence microscopy imaging. An area of the grid containing transfected cells was chosen and imaged on an epifluorescence light microscope equipped with a 20x air objective. Bright field images, GFP-channel and SFR channel images were acquired in order to re-identify the transfected cells positive for Bax-EGFP.

After fluorescence light microscopy, the sample sandwiches were disassembled and immersed in pre-warmed fixative consisting of fresh thawed $2.5 \%$ glutaraldehyde in $0.1 \mathrm{M}$ cacodylic buffer at $\mathrm{pH} 7.4$.

Samples were then processed for resin embedding and electron microscopy investigations. After curing of the resin, the area of interest was processed with a razor blade and finally ultrathin serial sections of $70 \mathrm{~nm}$ thickness of the area of interest were collected on Formvar coated slot grids.

Prior to detailed TEM investigations, the sections were exposed to the electron beam at low magnification (about 460x original magnification) and a widespread condenser setting and spot sizes between 3 or 4 for up to 15 minutes. This helps to increase the image contrast at lower magnifications. Overview images were obtained with an original magnification of 460x (Figure 27, white stars indicate a few selected positions of Bax-EGFP expressing cells as regions of interest in the fluorescence light microscopy image and the transmission electron micrograph respectively).

For subsequent detailed electron microscopy imaging, the light microscopy image was registered onto the electron micrographs obtained at low magnifications by using the cell bodies as well as the nuclei as intrinsic landmarks. This process was continued until an identification of each cell was possible. The characteristic shape of HeLa cells aids this process and a coarse overlay already indicated the position of each cell. Finally, the position of the light microscopy information in the overlaid images indicated areas for subsequent high-resolution TEM analysis. 
Electron micrographs at 1000x original magnification were recorded for a better alignment to identify subcellular regions of interest for detailed imaging (Figure 27 B). Intrinsic landmarks that are visible in both imaging modalities are very helpful to align the two $3 \mathrm{D}$ volumes. In this example, an area containing multiple mitochondria labelled with Mitotracker Deep Red FM together with two lysosomes was chosen in close proximity to multiple Bax-EGFP signals (Figure 27 C).
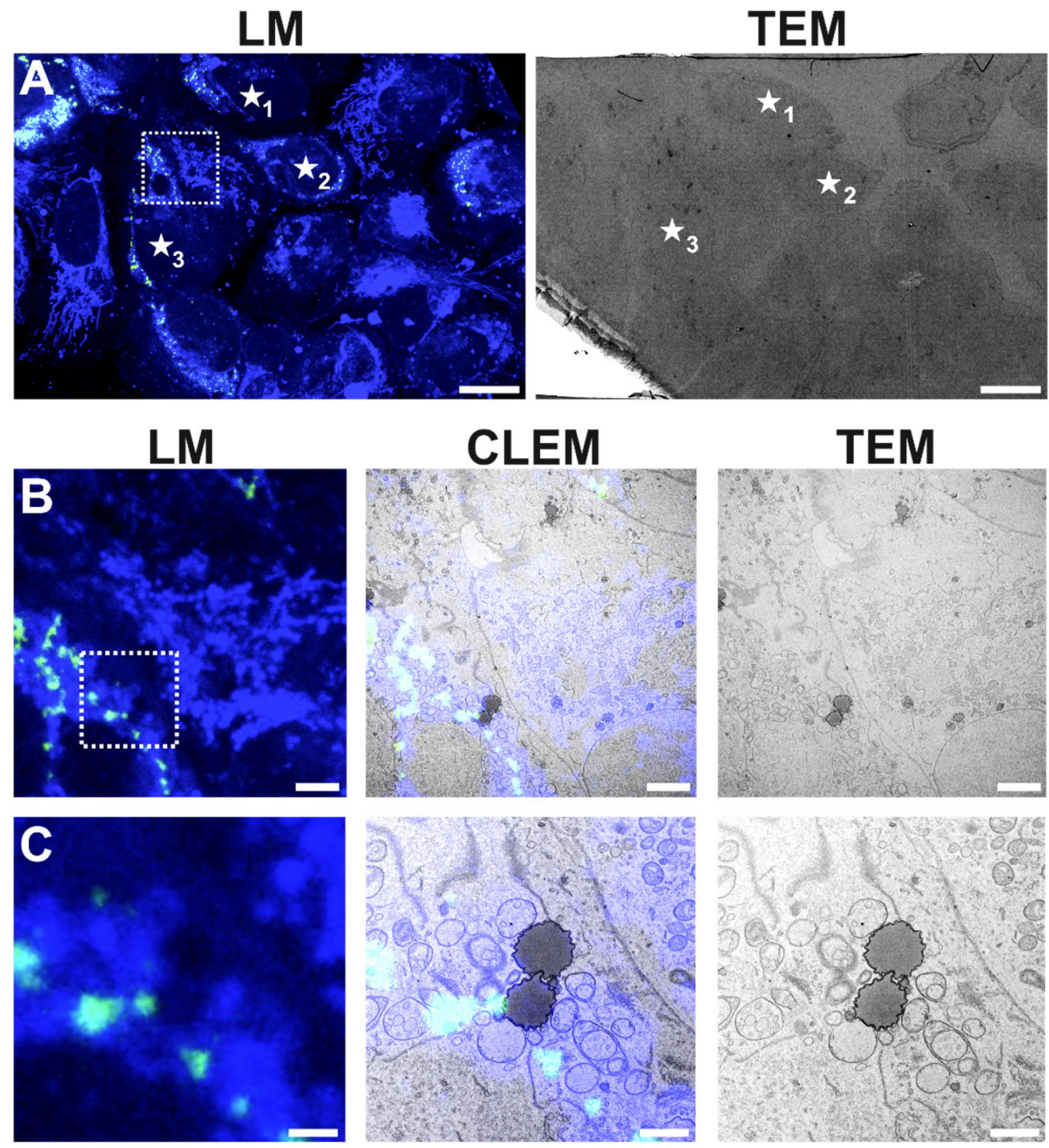

Figure 27: Live cell CLEM of EGFP-Bax expressing HeLa-cells. A: Low magnification images allow re-identification of the cells of interest. Channels in fluorescence microscopy: Blue: Mitotracker Deep Red FM. Green: Bax-EGFP. B: Medium magnification of an area of interest within a cell. $\boldsymbol{C}$ : Precise correlation defines function-structure relationship. Scale bars in $A=$ $20 \mu \mathrm{m}$. Scale bars in $B=2 \mu \mathrm{m}$. Scale bars in $C=1 \mu \mathrm{m}$.

The registration of both 3D volumes lead to a localization of the Bax-EGFP signal within each electron micrograph obtained by serial sectioning (SsTEM, Figure 28, see supplementary Figure 42 for the full stack). The signal of Bax-EGFP is in close proximity to five mitochondria that are swollen, rounded and show mitochondrial outer membrane ruptures (membrane 
ruptures are indicated with white arrows). An additional density that is possibly due to a high local protein concentration co-localizes exactly with the Bax-EGFP signal as recorded by fluorescence light microscopy (Figure 28 densities are indicated with white dotted outlines).
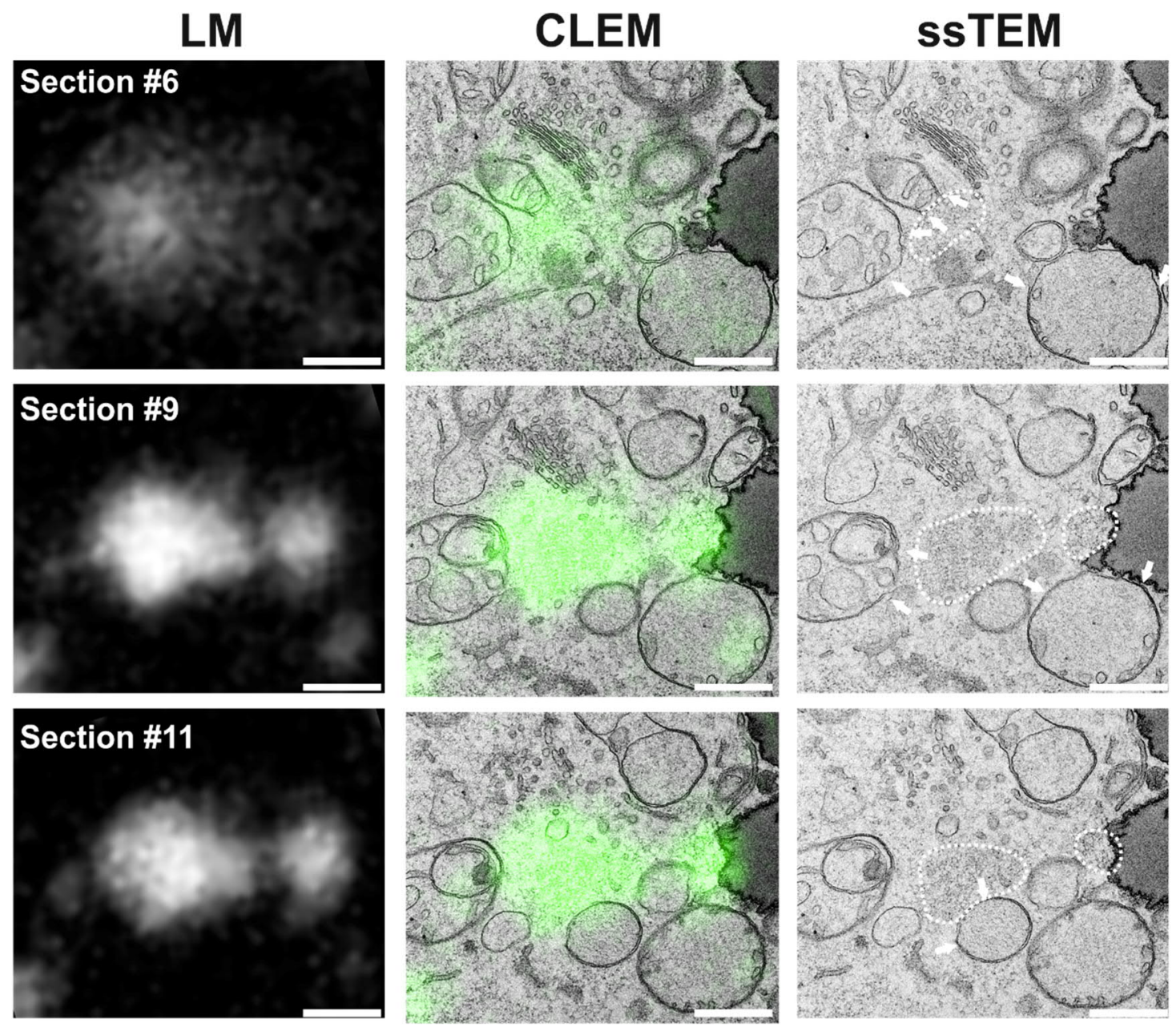

Figure 28: 3D-CLEM of mitochondria during apoptosis. Left column: selected optical sections of the fluorescence light microscopy stack showing the EGFP-signal. Middle column: CLEM overlay of corresponding registered images (green = EGFP signal). Right column: selected sections from serial sectioning TEM of the region of interest. White arrows indicate outer mitochondrial membrane ruptures. White dotted lines indicate high protein densities. Scale bars $=500 \mathrm{~nm}$.

Next, features identified in the electron micrographs such as mitochondrial membranes as well as the density in the centre of the images were subjected to manual segmentation using IMOD and were displayed as capped objects. The 3D mesh for the EGFP-signal was obtained by using the pixel threshold segmentation in microscopy image browser (164) (Figure 29).

Model meshes resulted in 3D representations of the mitochondrial outer membrane (dark blue) and the mitochondrial inner membrane (light blue, Figure $29 \mathrm{~A}$ ). Within these mitochondria, a large area of the mitochondrial outer membrane is missing. Thus, these sites were showing a herniation of the mitochondrial inner membrane into the cytoplasm. The EGFP-density 
localized in the centre with the mitochondrial inner membrane herniations facing it (Figure 29 $\mathbf{B}$ and $\mathbf{F}$ ). The model was then extended with the segmentation of the mesh-like density as identified in the electron micrographs (Figure $29 \mathbf{C}$ and E). This density as well was centred with respect to the mitochondrial inner membrane herniations. Intriguingly, both models did exactly overlap with each other indicating that the mesh-like density contained large amounts of Bax-EGFP (Figure 29 D).
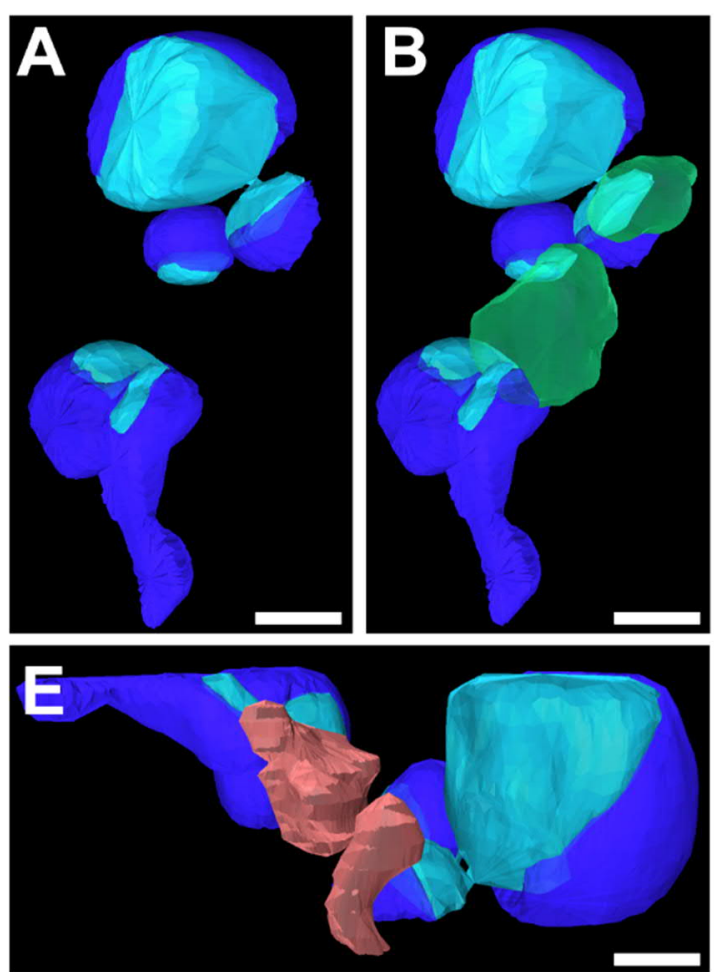
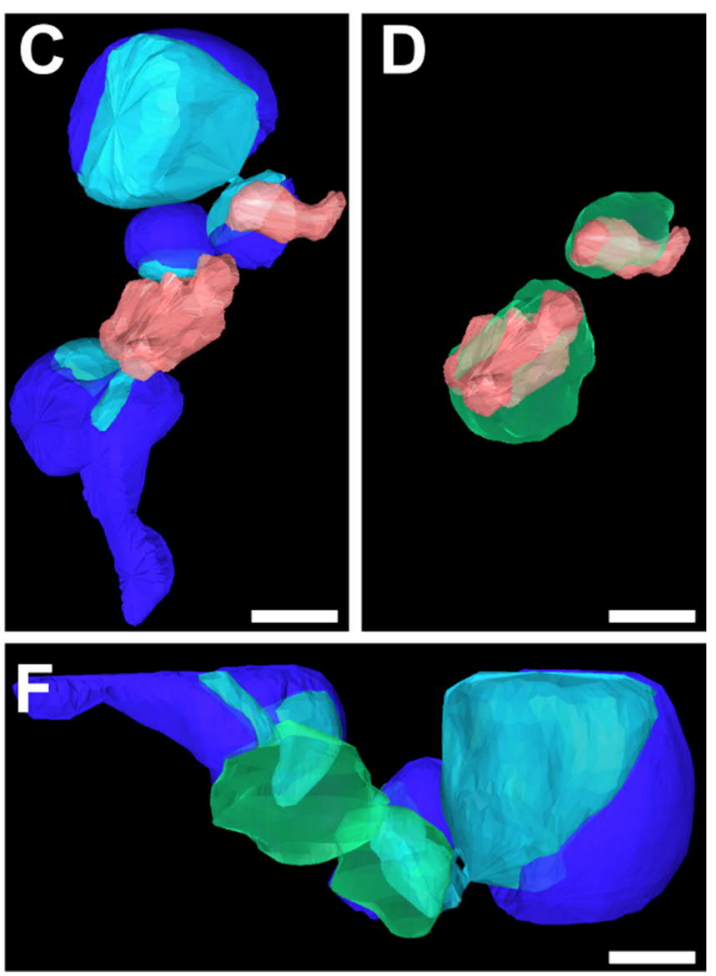

Figure 29: 3D reconstruction of apoptotic mitochondria as identified by CLEM. A: Materials of the outer mitochondrial membrane (dark blue) and inner mitochondrial membrane (light blue) showing ruptures of the outer membrane. B: Additional localization of the EGFP-signal (green) in the area of interest. $\boldsymbol{C}$ : Reconstruction of the mesh-like density (red). D: Overlay of the EGFP-signal and the reconstruction of the mesh-like density. E: Side-view of the model showing the mitochondria with the protein-rich density. F: Side-view of the model showing the mitochondria with the Bax-EGFP signal. Scale bars $=500 \mathrm{~nm}$.

In conclusion, the overexpression of Bax-EGFP induced intrinsic apoptosis in HeLa cells. In cells containing large clusters, the mitochondrial network was fragmented and showed localizations of Bax-EGFP clusters close to the mitochondria. Subsequent high-resolution TEM investigations revealed, that in close proximity to Bax-clusters, the outer mitochondrial membrane showed large ruptures accompanied with a herniation of the mitochondrial inner membrane. The application of live cell CLEM demonstrated that proteins fused to a fluorescent reporter could be investigated in context with their surrounding ultrastructure. The cells analysed with CLEM showed large clusters of Bax-EGFP that might indicate a late stage of apoptosis. It needs to be tested, whether the live-cell CLEM protocol presented here can be applied to identify and study earlier time points of apoptosis as well. 


\section{The temporal succession of BAX mediated apoptosis}

Progression of intrinsic apoptosis in cells is coupled to oligomerization of Bax on the mitochondrial outer membrane (165). Here, I used multiple stages of Bax-mediated apoptosis that have been identified by different cluster sizes of Bax-EGFP in light microscopy to test if my established live-cell CLEM protocols could be applied to study the temporal succession of biological events.

To this end, HeLa cells were transfected with BAX-EGFP according to the previously described protocol. Finder-substrates containing expressing cells were picked after 4, 5, 6 and 7 hours post-transfection and areas containing BAX-EGFP expressing cells showing different sizes of Bax-EGFP clusters were selected in light microscopy and prepared for transmission electron microscopy. Ultra-thin sections of $70 \mathrm{~nm}$ thickness were collected on Pioloform-coated slot grids and examined at $8600 x$ original magnification. Cells were identified based on the light microscopy data.

Cells without expression of Bax-EGFP feature wildtype-ultrastructure of the mitochondria (Figure $30 \mathrm{~A}$ ) with the crista-membranes being in a lamellar arrangement. With the localization of Bax to the mitochondrial outer membrane, the mitochondrial network fragments. The mitochondrial matrix-spaces started to separate and the crista membranes are already less oriented (Figure $\mathbf{3 0} \mathbf{B}$, open spaces are indicated with a white star).
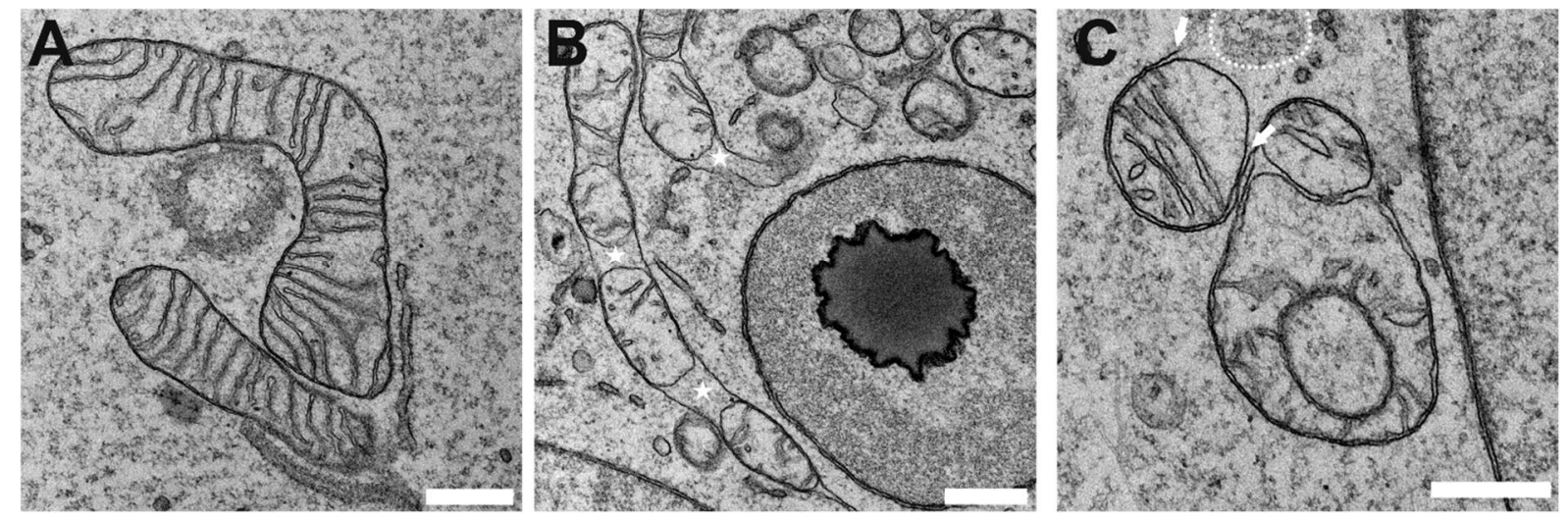

Figure 30: Temporal succession of Bax-mediated apoptosis in HeLa cells analysed with transmission electron microscopy. A: Cells without Bax expression have wildtype mitochondria. B: Mitochondria in the early stage of apoptosis. Mitochondria are elongated but the matrix space started to separate as indicated with white stars. C: Mitochondria in the late stage of apoptosis. Mitochondria are rounded and a subset showed ruptures of the outer mitochondrial membrane. Opening of the outer membrane is indicated with white arrows. $A$ protein-rich density is indicated with a white dotted line. Scale bars $=300 \mathrm{~nm}$.

At later stages, the mitochondrial network starts to fragment into small individual mitochondria that locate close to the nucleus. This process was already described as the perinuclear collapse of mitochondria during apoptosis (166). At late apoptotic stages, a sub-fraction of mitochondria features a clear rupture of the outer membrane (Figure $30 \mathbf{C}$, mitochondrial outer 
membrane rupture is indicated with white arrows). Moreover, at higher expression levels of Bax-EGFP, a mesh-like density around membrane rupture sites could be detected in the electron micrographs (Figure $\mathbf{3 0} \mathbf{C}$, the density is indicated with a white dotted line).

In summary, the intrinsic apoptosis of HeLa cells mediated by overexpression of Bax-EGFP ultimately results in the rupture of the mitochondrial outer membrane. In light microscopy investigations, a ring-like organization of Bax clusters on the mitochondrial membrane was shown (162, 167). However, the underlying membrane architecture of these mitochondria could only be investigated by using correlative light and electron microscopy. These results demonstrated that correlation of light and electron microscopy could be used to dissect biological processes in a time dependent manner. Here, the complex membrane remodelling of mitochondria during intrinsic apoptosis could be linked to the activity of Bax.

However, the registration of light microscopy images to electron micrographs can be challenging in the live-cell CLEM approach presented here since sample preparations for electron microscopy that occurs after light microscopy investigations can introduce perturbations in the sample. These perturbations caused by dehydration and resin embedding of the sample can limit the precision of the data registration. In addition, sufficient landmarks are needed to correlate both data sets with high precision especially in 3D. This problem could be addressed with a sample preparation strategy that allows recording fluorescence images and electron micrographs from the same resin embedded specimen. This approach is called post embedding CLEM or high-accuracy CLEM and a protocol establishment is given in the following.

\section{Workflow for high-accuracy CLEM}

The workflow of live cell CLEM (pre-embedding CLEM) can induce significant and structural perturbations that lead to limitations in the final data registration $(117,118)$. In order to address this challenge, the whole sample preparation can be completed before any imaging takes place and the samples are stained and embedded in a resin suitable for both, fluorescence and electron microscopy.

Prior to this work, a variety of approaches and protocols for high accuracy CLEM were already established and published $(168,169)$. A central aspect of these protocols is the immobilization of the cells by means of high-pressure freezing with subsequent freeze substitution and embedding in Lowicryl HM20 resin. In these preparations, the fluorescence of standard fluorescent proteins is preserved $(141,142,148)$. Thin sections of the resin-embedded cells can be viewed first in the light microscope and subsequently examined in electron microscopy. This approach is termed post-embedding CLEM or high-accuracy CLEM. 
Based on the published protocols of the group of Rainer Kaufmann (168) I implemented a workflow for high-accuracy CLEM. Major differences of my protocol compared to the published protocol include the abolishment of tannic acid in the freeze substitution cocktail and changes to the timing and temperature during resin infiltration.

To implement a protocol for high-accuracy CLEM, HeLa cells were grown on $10 \mathrm{~cm}$ cell culture dishes. The cells were incubated with a final concentration of $200 \mathrm{nM}$ Mitotracker Deep Red FM 30 min prior to detachment from the cell culture dishes.

The cells were detached from the cell culture dishes using $0.25 \%$ pre-warmed trypsin in PBS. The cells were then collected in a falcon tube and trypsin de-activated using double volume of pre-warmed DMEM complete medium. Subsequently, cell pellets were obtained by centrifugation at $300 \mathrm{~g}$ for 3 minutes. The pellets were re-suspended in pre-warmed DMEM without phenol red, supplemented with an additional $25 \mathrm{mM} \mathrm{HEPES}$. These samples were kept in a $1.5 \mathrm{ml}$ Eppendorf tube on a heating block set to $35^{\circ} \mathrm{C}$ until freezing. For the freezing of the cells, a paste-like consistency of the sample was achieved with a table top-centrifuge.

Finally, $2 \mu \mathrm{l}$ of this dense cell suspension was loaded in gold-coated copper-planchettes and the cells immobilized by high-pressure-freezing. This not only provided an excellent preservation of the membranous ultrastructure but also retained the fluorescence properties of any dyes added or fluorescent fusion proteins expressed prior to the immobilization (169171).

After freeze substitution and staining of the cell material with uranyl acetate and subsequent washing and resin infiltration, the resin was cured under UV light exposure resulting in final resin blocks that could be further processed at room temperature. Refer to the method section "Freeze substitution and resin embedding for CLEM" for details of the exact procedure.

For an initial screen for sample quality, the whole blocks were viewed with a 10x or 20x air objective and examined for fluorescence retention. Blocks showing preservation were then subjected to ultra-thin sectioning using a $35^{\circ}$ DiATOME diamond knife. Section of $120 \mathrm{~nm}$ thickness were collected on Formvar-coated TEM finder grids and stored in the dark until examination. Grids should be imaged as soon as possible since the fluorescence fades in the thin sections over 24 hours (data not shown).

Prior to light microscopy, the grids were dipped a couple of times in PBS in order to release any air-bubbles trapped on the backside of the grid. Then, the grids were placed within a drop of PBS that has been placed on a glass microscope slide with the side facing upwards where the sections have been immobilized. The grids are then covered with a glass cover slip and observed with a fluorescent light microscope. In order to retrieve the areas of interest, the 
corresponding grid boxes containing the cells were imaged in the brightfield mode. The additional imaging of the DAPI channel resulted in an image representing the overall cell structure as uranyl acetate served here as a dual-purpose contrasting agent for both, light and electron microscopy (172). Finally, the channel corresponding to the protein of interest was imaged. This approach was continued over multiple areas of the grid to maximize the amount of data that could be obtained from a single gird.

After fluorescence microscopy imaging, the grids were recovered from the microscope slide. This was achieved by pipetting PBS to the edge of the glass coverslip in order to let the coverslip gently float-off from the microscope slide. The grid sticking to either side could be floated off as well by gently pipetting PBS next to the grid. Prior to electron microscopy imaging, the grids were washed multiple times on drops of double distilled water.
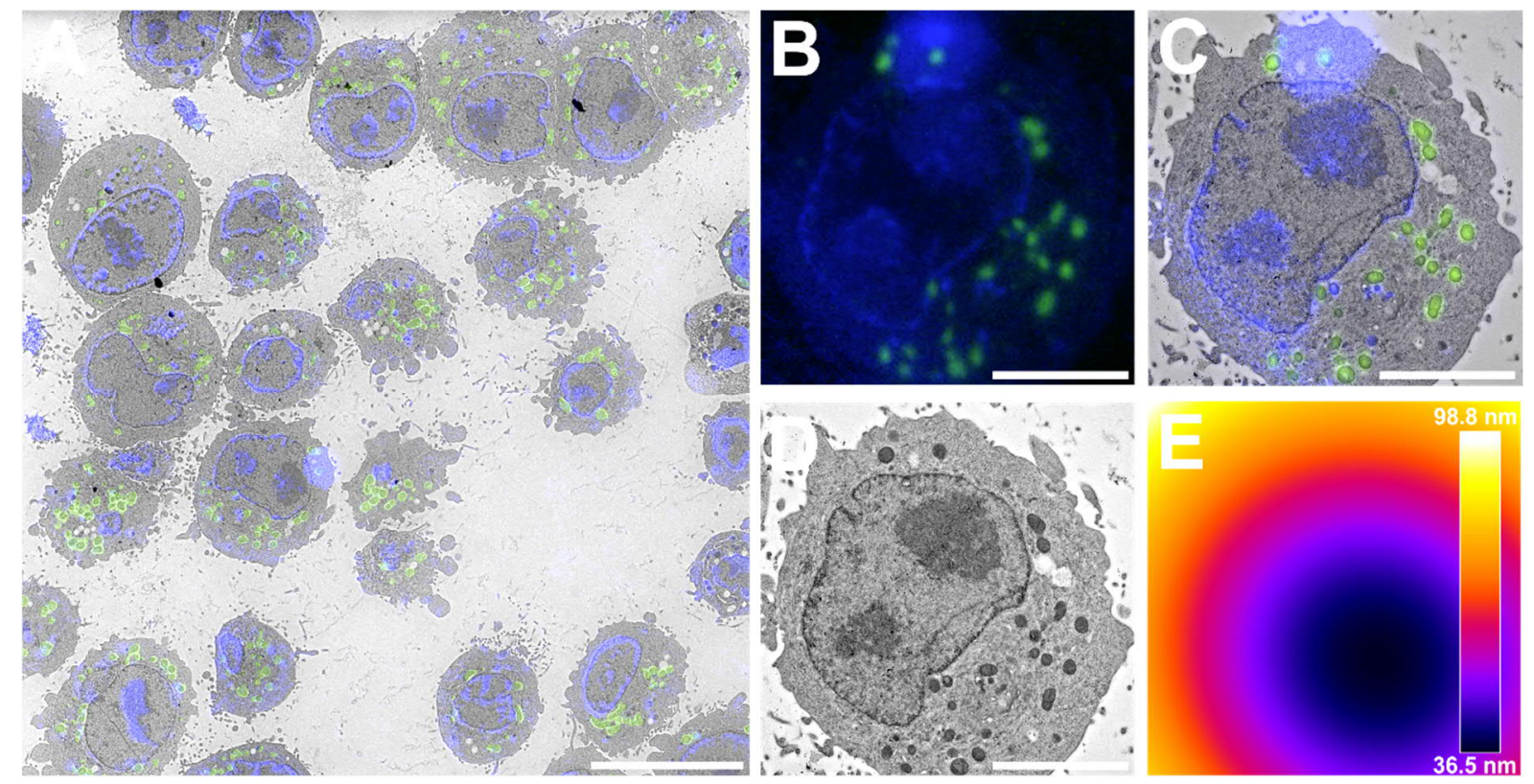

Figure 31: High-accuracy CLEM. A: Final CLEM-map to target cells of interest. B: Fluorescence light microscopy image of a cell of interest, blue represents uranyl acetate phosphorescence imaged in the DAPI channel, green represents Mitotracker Deep Red FM imaged in the far-red channel. C: Registered Images of fluorescence light microscopy to the electron micrograph highlighting regions of interest. $\boldsymbol{D}$ : Transmission electron micrograph from the same cell shown in $B$. E: Error-map for the $2 D$-linear transformation of $B$ onto $D$. Scale bar in $A=15 \mu \mathrm{m}$. Scale bars in $B, C$ and $D=5 \mu \mathrm{m}$.

The grids were then transferred for examinations with transmission electron microscopy. The regions of interest were identified by using the index of the grid and overview images acquired at $120 \mathrm{kV}$ with $460 \mathrm{x}$ original magnification. Multiple images were acquired in order to cover the entire grid boxes. The overview images were stitched in Photoshop CS 6 with the "Photomerge" automated procedure. In the following, overview images obtained by fluorescence light microscopy were registered to the stitched electron micrographs in ICY with the plug-in eC-CLEM (152) (Figure 31). The phosphorescence of uranyl acetate depicts the 
overall cell shape. Furthermore, it highlights especially phosphorous-rich compartments. This can be seen as a highlight of the heterochromatin giving the shape of the nucleus in the fluorescence microscopy images. This channel can now be used to identify the cells within the grid box, as the shape of the nucleus is unique for every cell. Furthermore, this information can be used as intrinsic landmarks for image registration (172).

All image channels obtained by fluorescence light microscopy were adjusted manually and individually in order to give clear visibility of the features. The resulting CLEM-map can then be used to identify cell-areas of interest that can be subsequently imaged in electron microscopy at higher magnifications. In this example, the sections were imaged at $120 \mathrm{kV}$ with $3500 x$ original magnification. This results in areas within the cell where proteins of interest can be localized with a registration error between 40 to $100 \mathrm{~nm}$ within the corresponding electron micrograph (Figure $31 \mathrm{E})$.
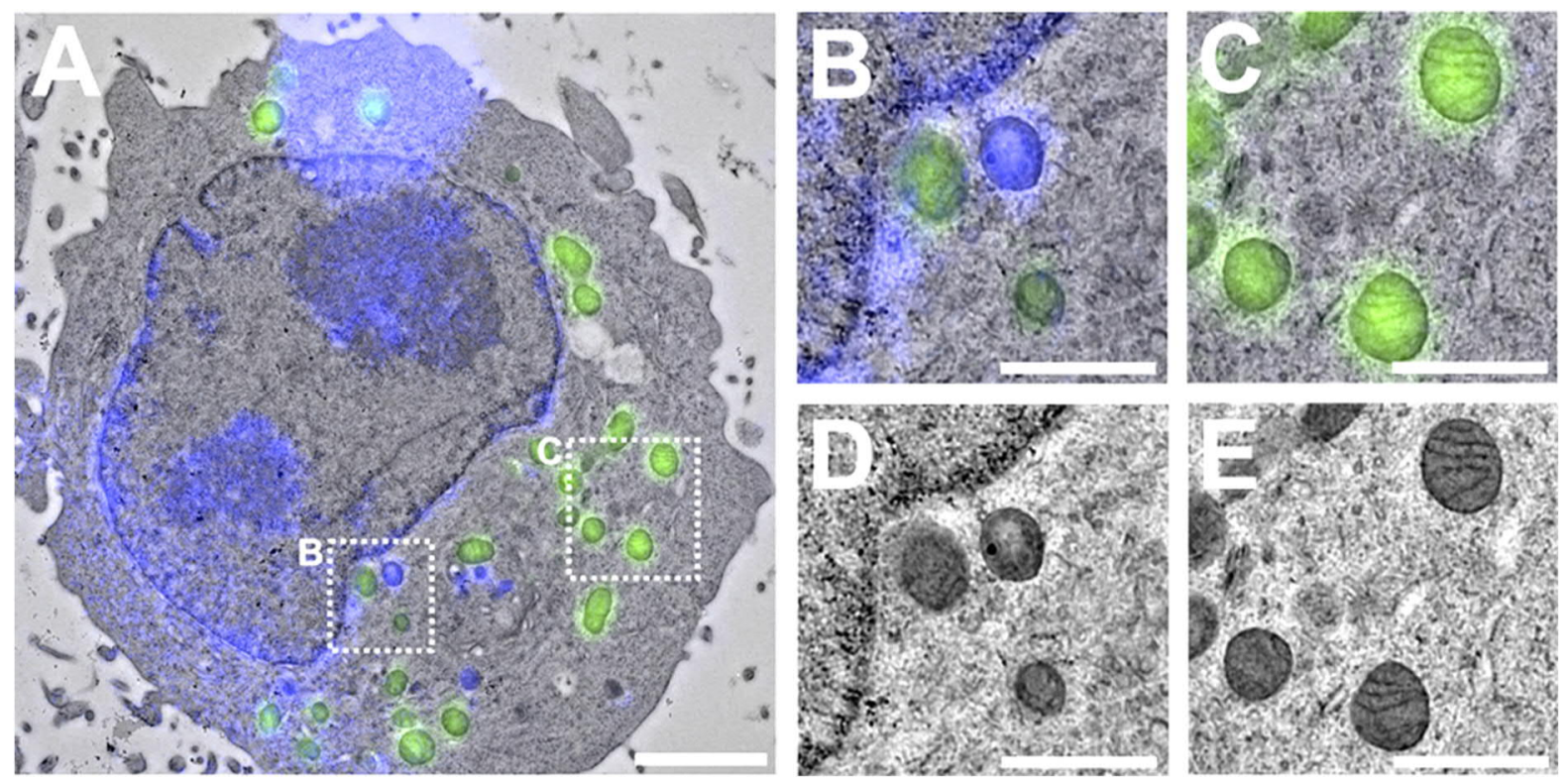

Figure 32: High-accuracy CLEM in detailed view. A: Registered overlay of a fluorescence light microscopy image to the corresponding electron micrograph. B: CLEM - Zoom-in on nucleus, mitochondria and a multi-vesicular-body as identified in A. C: CLEM - Zoom-in on mitochondria as identified in A. D: TEM corresponding to B. E: TEM corresponding to C. Scale bar in $A=2$ $\mu \mathrm{m}$. Scale bars in $B, C, D$ and $E=1 \mu \mathrm{m}$.

High-accuracy CLEM can be employed to analyse subcellular areas (Figure 32). A bright spot imaged within the DAPI channel localizing on a structure that might be similar to an endosome that is enriched in uranyl acetate stain possibly due to a high phosphorus content (Figure 32 $\mathbf{B}$ and $\mathbf{D})$. Detailed images show a precise localization of the signal obtained from imaging Mitotracker Deep Red FM that indeed corresponds to mitochondria as seen in the electron micrographs (Figure $32 \mathbf{C}$ and $\mathbf{E}$ ). 
In summary, correlative imaging of light microscopy and electron microscopy was applied to reveal the functional and structural relation of cellular details. This was demonstrated first in the correlation of Bax-EGFP imaged in live cells with subsequent detailed electron microscopy investigations. A complex mitochondrial membrane remodelling in close proximity to Bax clusters was shown in 3D. Next, a workflow for high accuracy localization of light microscopy images to electron micrographs was enabled with the establishment of in-resin CLEM. A localization precision of $<100 \mathrm{~nm}$ was demonstrated by imaging mitochondria stained with Mitotracker Deep Red FM first in fluorescence light microscopy with a subsequent visualization of the same mitochondria in transmission electron microscopy. Additionally, for in-resin CLEM fluorescent proteins expressed in HeLa cells were tested (supplementary Figure 41). CLEM is used to study proteins of interest in their corresponding ultrastructural context. However, neither light nor electron microscopy can address the chemical composition and turnover of cellular structures. Mass spectrometry is widely used to analyse turnover in cells. However, after processing and homogenization of the cells, the spatial information is lost. Nanoscale secondary ion mass spectrometry offers quantitative analysis of secondary ions with spatial precision similar to conventional light microscopy. A correlation of NanoSIMS with highaccuracy CLEM would result in a comprehensive understanding of cellular features. First, a workflow for NanoSIMS-correlation to TEM is needed to test for the feasibility of such a protocol

\section{Expanding the information obtained by correlative imaging}

Nanoscale secondary ion mass spectrometry (NanoSIMS) allows investigating the chemical composition of cells while retaining the spatial information. A primary ion beam such as $\mathrm{Cs}^{+}$or $\mathrm{O}^{-}$is scanned across the sample. The collision with biological material leads to the erosions of a few nanometres on the surface. This result in secondary ions derived from cellular structures that are then guided by ion optics to a mass spectrometer where they can be analysed. The beam is scanned across the sample and secondary ions collected for each position. This results in a mapping of the abundance of each secondary ion. The chemical composition of the sample can then be analysed. Further, feeding cells with heavy isotopes will reveal the turnover of biomolecules at specific positions. However, in NanoSIMS, only the secondary ions are imaged. To identify organelles a correlation to either light microscopy or electron microscopy is needed.

To establish a TEM-NanoSIMS correlative imaging protocol, HeLa cells were used. In this preliminary experiment, no heavy isotope labelling was employed and only the natural abundance of certain isotopes in the cell were analysed. 
To this end, HeLa cells were seeded in a $10 \mathrm{~cm}$ cell culture dish at approximately $80 \%$ confluency. On the following day, the cells were detached from the culture dish by using 0.25 $\%$ trypsin in PBS ( $\mathrm{pH} \mathrm{7.4)}$ and collected in a falcon tube. The enzyme was deactivated by adding the double amount of pre-warmed DMEM complete medium to the cell suspension. The cells were then centrifuged at $300 \mathrm{~g}$ for 5 minutes and re-suspended in $1 \mathrm{ml}$ DMEM complete medium further supplemented with 25 mM HEPES and kept on a heat block set at $35^{\circ} \mathrm{C}$ until immobilization by high-pressure freezing. Samples were then prepared via freeze substitution and subsequent resin embedding in Lowicryl HM20. For transmission electron microscopy examinations, $90 \mathrm{~nm}$ thin sections were prepared and collected on Formvar- and carbon-coated finder grids and imaged at $120 \mathrm{kV}$ with an original magnification of $6500 \mathrm{x}$. Grid boxes were imaged in a 10x10-tile format in order to cover many cells for subsequent metabolic imaging. The images of cells of interest were stitched automatically with Photoshop CS 6 and the "Photomerge" automated procedure. Subsequently, the samples were transferred to the NanoSIMS imaging and the abundance of the following ions was measured: ${ }^{12} \mathrm{C}^{14} \mathrm{~N}^{-},{ }^{12} \mathrm{C}^{15} \mathrm{~N}$, ${ }^{31} \mathrm{P}^{-}$and ${ }^{32} \mathrm{~S}$.
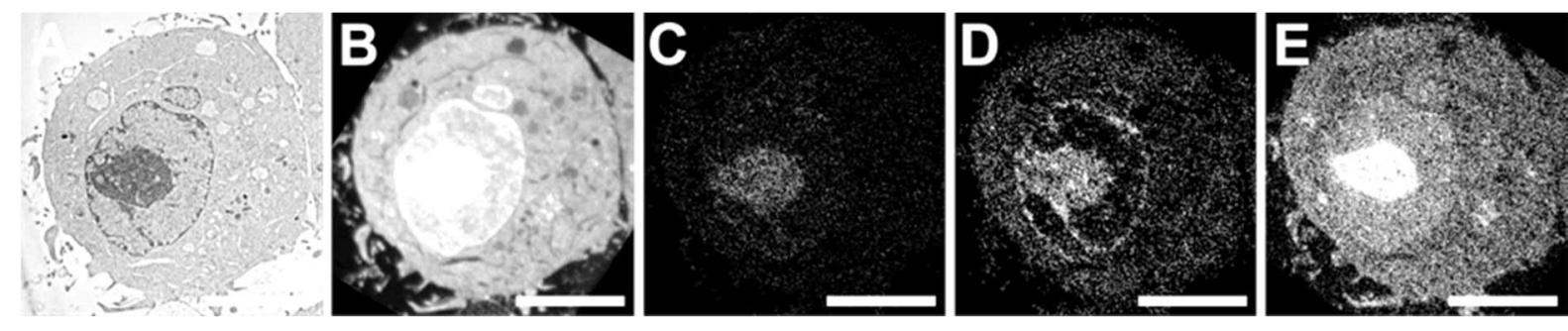

Figure 33: Transmission electron microscopy correlated to NanoSIMS. A: Transmission electron micrograph of a HeLa cell. B: Abundance of the ${ }^{12} \mathrm{C}^{14} \mathrm{~N}^{-}$ion, C: Abundance of the ${ }^{12} \mathrm{C}^{15} \mathrm{~N}^{-}$ion. D: Abundance of the ${ }^{31} \mathrm{P}^{-}$ion. $\mathrm{E}$ : Abundance of the ${ }^{32} \mathrm{~S}^{-}$ion. Scale bars $=10 \mu \mathrm{m}$.

Transmission electron microscopy images and NanoSIMS images were registered using 2D linear transformations in the ICY imaging analysis programme (Figure 33). Uranyl acetate was used as a contrasting agent for electron microscopy. This reagent stains primarily proteins in the cell. Thus, cellular structures enriched in proteins such as the heterochromatin appear with high contrast in the electron micrographs. ${ }^{12} \mathrm{C}^{14} \mathrm{~N}$ ions derive from proteins in the sample. High intensities of this ion correspond to a high local protein concentration. In that sense, the electron micrograph and the ${ }^{12} \mathrm{C}^{14} \mathrm{~N}$ - ion contain similar information. Therefore, the ${ }^{12} \mathrm{C}^{14} \mathrm{~N}^{-}$ion channel is very useful for data correlation and image registration (Figure $33 \mathbf{B}$ ).

The abundance of the ${ }^{12} \mathrm{C}^{15} \mathrm{~N}$ ion is expected to be low since no heavy isotope labelling was employed, thus only the natural abundance of the isotope is to be expected (around $1.07 \%$, Figure $33 \mathrm{C}$ ). Recording the abundance of the ${ }^{31} \mathrm{P}^{-}$ion showed an enrichment in dense areas of the nucleus that might be heterochromatin (Figure $33 \mathbf{D}$ ). The recorded abundance of the ${ }^{32} S^{-}$ion showed a strong enrichment in the dense centre part of the nucleus as well as in a few 
areas that show dense granules that might be multi vesicular bodies (Figure $33 \mathbf{E}$ ). Another observation is the low abundance of ${ }^{12} \mathrm{C}^{14} \mathrm{~N},{ }^{31} \mathrm{P}^{-}$and ${ }^{32} \mathrm{~S}^{-}$in structures that are similar to early lysosomes as seen in the electron micrograph (Figure 33).

The correlation of transmission electron microscopy to NanoSIMS of the same resin sections of cells allowed a precise understanding of the data obtained by metabolic imaging. This is especially true since cellular structures are not readily identified in NanoSIMS measurements. Another benefit is the much better lateral resolution of transmission electron microscopy, which, in resin-embedded biological samples, is usually at around $10 \mathrm{~nm}$ as opposed to around 80-160 $\mathrm{nm}$ in NanoSIMS.

Structures in NanoSIMS could also be identified by using light microscopy prior to SIMS measurements. However, a correlation of all three techniques on one single sample would allow the recording of functional and structural information in combination with the chemical composition for sub-cellular areas. Therefore, a combination of protocols for high-accuracy CLEM with NanoSIMS is needed.

\section{Correlative light, electron and ion microscopy}

To establish a workflow to correlate light microscopy, electron microscopy and metabolic imaging on the same resin section, HeLa cells were seeded in a $10 \mathrm{~cm}$ cell culture dish at approximately $80 \%$ confluency. On the following day, the cells were incubated with a final concentration of $200 \mathrm{~nm}$ Mitotracker Deep Red FM for 30 minutes to stain mitochondria for light microscopy. Subsequently, the cells were immobilized by high-pressure freezing, resin embedded and processed for high-accuracy CLEM. Thin-sections of the final resin blocks were prepared with a thickness of $160 \mathrm{~nm}$, collected on commercial Formvar/carbon coated TEM finder grids (TEM Grids, Formvar-Karbonfilm beschichtet, 200 Mesh, Gilder Finder F1, Science Services, Munich, Germany), and immediately examined in an epifluorescence microscope with the procedure described in the "high-accuracy CLEM" section.

Overlapping images of all channels of interest were manually recorded and finally stitched together with the automated Photoshop CS6 procedure "Photomerge". Overview images in transmission electron microscopy were recorded at $120 \mathrm{kV}$ with $460 \mathrm{x}$ original magnification. These overview images served to create a CLEM-map for detailed imaging. Detailed images of the cells were recorded in TEM at $120 \mathrm{kV}$ with $3500 \mathrm{x}$ original magnification and multiple images of a cell stitched together with the automated Photoshop CS6 procedure "Photomerge". 
The samples were then transferred for NanoSIMS measurements and cells of interest were analysed for the abundance of several naturally occurring ions and elements (Figure 34).

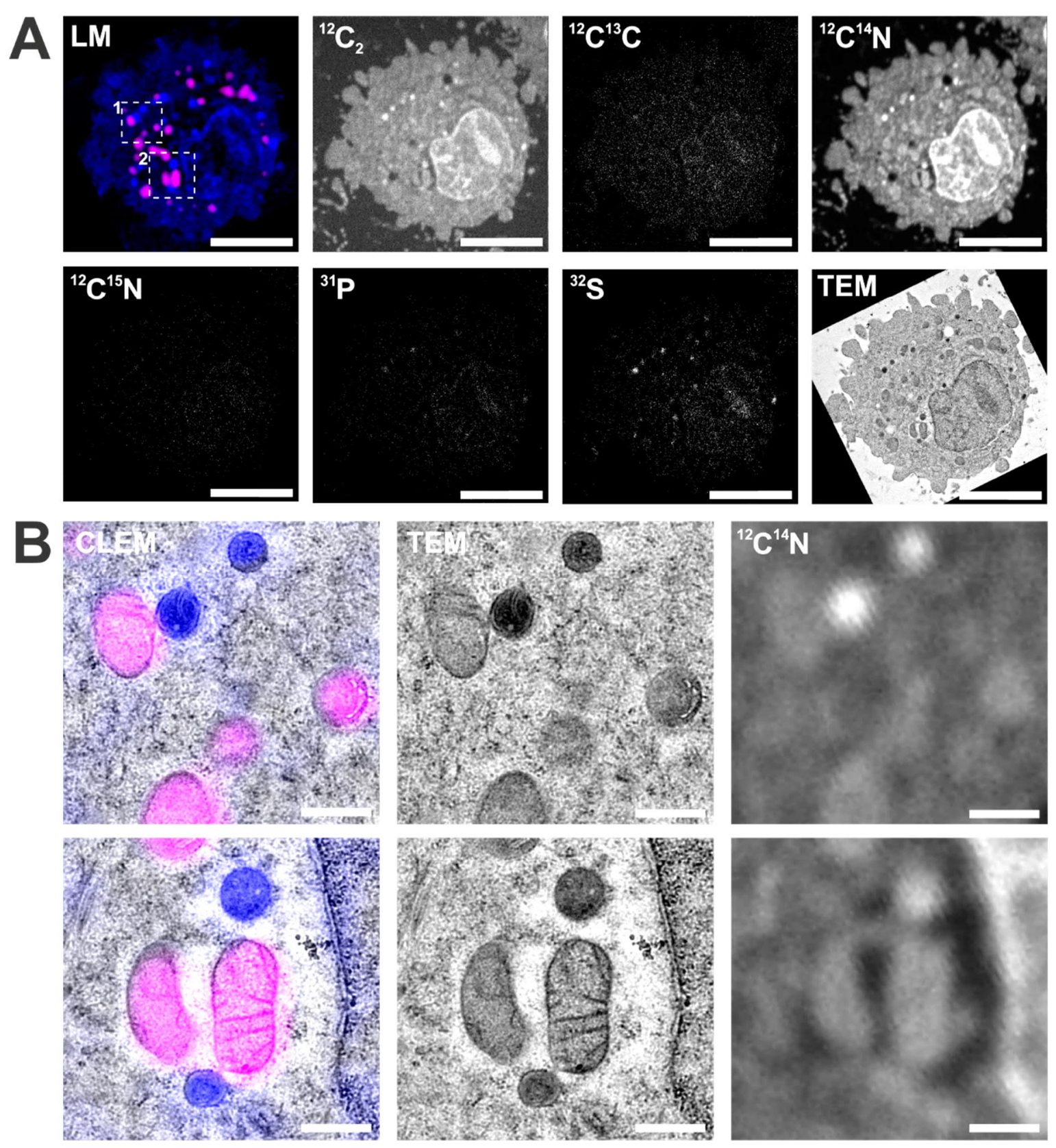

Figure 34: CLEM-SIMS of HeLa cells. A: Information obtained by the three imaging modalities on the same cell. LM: light microscopy of the DAPI channel (blue) and Mitotracker Deep Red FM (magenta). ${ }^{12} \boldsymbol{C}_{2},{ }^{12} \boldsymbol{C}^{13} \boldsymbol{C},{ }^{12} \boldsymbol{C}^{14} \boldsymbol{N},{ }^{12} \boldsymbol{C}^{15} \mathbf{N},{ }^{31} \mathrm{P}$ and ${ }^{32} \mathrm{~S}$ ion abundance recorded with NanoSIMS. TEM: transmission electron micrograph of the cell of interest. B: Detailed images of CLEM, TEM and ${ }^{12} C^{14} N^{-}$ion signal from the boxed regions 1 and 2 in $A$. Scale bars in $A=5$ $\mu \mathrm{m}$. Scale bars in $B=500 \mathrm{~nm}$ (145).

Light microscopy images and electron micrographs presented in Figure 34 A were registered by linear transformation to the ${ }^{12} \mathrm{C}^{14} \mathrm{~N}$ ion channel. CLEM images presented in Figure $34 \mathbf{B}$ were obtained by linear transformation of the light microscopy images to the electron micrograph. The ${ }^{12} \mathrm{C}^{14} \mathrm{~N}^{-}$ion channel presented in Figure 34 B was registered by liner 
transformation to the electron micrograph as well. Measurements with NanoSIMS with the associated analysis was provided by Paola Agüí González in the laboratory of Prof. Dr. Silvio Rizzoli. Details about the data acquisition and the image analysis can be found in the published manuscript (145).

The analysis revealed, that mitochondria labelled with Mitotracker Deep Red FM, are enriched in protein compared to the cytosol. Multivesicular bodies are especially rich in protein content, sulphur and phosphorus. Lysosomal vesicles are low in protein content as compared to the cytosol. The heterochromatin is enriched in protein, sulphur and phosphorus pissibly due to the dense packaging of the chromatin. Intensities of the ${ }^{12} \mathrm{C}^{15} \mathrm{~N}^{-}$and ${ }^{12} \mathrm{C}^{13} \mathrm{C}$ ions is low due to their low natural abundance.

The correlation of fluorescence microscopy to transmission electron microscopy and ion microscopy presented in this chapter resulted in a workflow to visualize specific labelled cellular components, their structural context and analysis of their chemical composition. This protocol provides information about sub-cellular areas that are inaccessible with conventional correlative approaches.

Taken together, in this thesis I developed techniques for correlative imaging using fluorescence and electron microscopy. These protocols could be used to correlate a live-cell observation by fluorescence microscopy to the corresponding ultrastructure or to identify the position of a protein of interest within the structural context at high precision. Further, a correlation of either transmission electron microscopy or high-accuracy CLEM to metabolic imaging was demonstrated. The protocols presented in this thesis provide a solid basis to study the mitochondrial adaptation in cultured neurons in future experiments. 


\section{Discussion and outlook}

\section{Section 1 - Mitochondrial adaptation in the neuronal presynapse}

Mitochondria contribute significantly to the generation of ATP in most eukaryotic cells. However, the adaptation of mitochondria at the neuronal presynapse during synaptic activity is enigmatic. In this work, I investigated the distribution and ultrastructure of presynaptic mitochondria and their structural adaptations to increasing energy demands using electron microscopy.

\section{Influence of the carbon source on mitochondrial membrane ultrastructure in cancer cells}

The mitochondrial ultrastructure might reflect the activity of mitochondria and thus, the amount of ATP that is produced. In cellular context, it is challenging to understand the precise ratio in ATP derived from either glycolysis or mitochondrial respiration. To minimize the amount of ATP derived from glycolysis, chemical inhibitors have been frequently used in the past (173). Irreversible Inhibitors include iodoacetic acid (IA) and iodoacetamide (IAA) that affect the enzyme glyceraldehyde-3-phosphate dehydrogenase (GAPDH) (174, 175). A standard agent for reversible inhibition of glycolysis is 2-deoxy-glucose (176). Both classes of compounds can be used to eliminate the short-time contribution of glycolysis to the cellular pool of ATP. However, irreversible chemical inhibition of glycolytic enzymes may ultimately lead to cellular stress and cell death (177). Furthermore, the inhibition of glycolysis would automatically reduce the levels of cellular pyruvate, thus, also affect mitochondrial respiration of the cells.

The rate of mitochondrial respiration can also be manipulated by chemical inhibitors (178). Rotenone is a widely used chemical compound that inhibits complex I in the mitochondrial chain reaction (179). Consequently, no ATP will be provided by mitochondrial respiration. However, similar to glycolysis inhibitors, mitochondrial inhibitors have also been shown to promote cell stress and cell death $(180,181)$. Hence, these tools can be used to study the short-term consequence of inhibition of either routes to generate ATP in the cell. However, they are insufficient to study cellular adaptation to increased rates of glycolysis or mitochondrial respiration respectively over longer time periods.

In order to analyse the long-term structural changes in mitochondria during changes in metabolic activity of the cells, I cultivated cells in presence of different carbon substrates. Control cells were cultivated in presence of high concentrations of glucose $(30 \mathrm{mM})$. These cells relied on glycolysis and mitochondrial respiration for the generation of ATP. Sodium- 
pyruvate and beta-hydroxybutyrate are substrates, which are exclusively used for ATP production via mitochondrial respiration. These two compounds were used in previous studies to investigate the mitochondrial adaptation to increased activity of respiration (182-186).

Cancer cells grown in DMEM medium lacking serum showed no adaptation of the mitochondrial membrane architecture in dependency of the available carbon source. Instead, high degrees of cell detachment and cell death were observed. This may have been caused by serum starvation of the cells over long time, which was reported to induce cell death (187189).

In the first experiments, cells cultivated in presence of beta-hydroxybutyrate showed a particularly high degree of cell death. Beta-hydroxybutyrate must be oxidised in the mitochondria to yield acetoactetyl-CoA (AcAc-CoA). AcAc-CoA can then be used for mitochondrial respiration and the synthesis of new biomolecules like lipids and DNA bases $(190,191)$. Cancer cells however, primarily utilize glutamine to fuel the citric acid cycle (156). Recent studies suggest that the cellular use of beta-hydroxybutyrate is low when other substrates for mitochondrial respiration are readily available (192 142). In the first experiment, the cells might have relied primarily on glutamine to fuel the tricarboxylic acid cycle. Glutamine cannot be converted into Ac-CoA thus, in this conditions no lipids or DNA bases can be synthesised. This might explain the lower vitality of cells cultivated in this condition, as they were not able to maintain the replenishment of biomolecules. Further, it explains the structural similarity of mitochondrial membranes in the other conditions (glucose and sodium-pyruvate) because glutamine was available in all conditions tested. To study the impact of the carbon source directly, glutamine was abolished from the cultivation medium in the following experiments.

Numerous studies on the biological effect of ketone bodies were published (193-197). Important findings include neuroprotective properties and the prevention of age-associated pathologies during the metabolism of ketone bodies (186, 193-195). However, the response of cellular and mitochondrial structure to ketone bodies has not been studied so far. Using electron microscopy, my work provides a detailed analysis of long-term mitochondrial plasticity in response to metabolic changes. Here, it was shown for the first time that mitochondria undergo significant changes in size and a complex crista membrane remodelling depending on the available carbon source of the cells (Figure 35).

In conclusion, cancer cells cultivated in medium containing glucose but lacking glutamine indeed primarily rely on glycolysis to generate ATP. The mitochondrial membrane architecture in these cells might represent a metabolically inactive form of mitochondria (1 in Figure 35). Interestingly, a similar mitochondrial membrane architecture has been previously shown for 
gametes in connection with high rates of glycolysis and low rates of oxidative phosphorylation in early developmental stages (198), thus supporting this notion.

\section{Cancer cell metabolism}

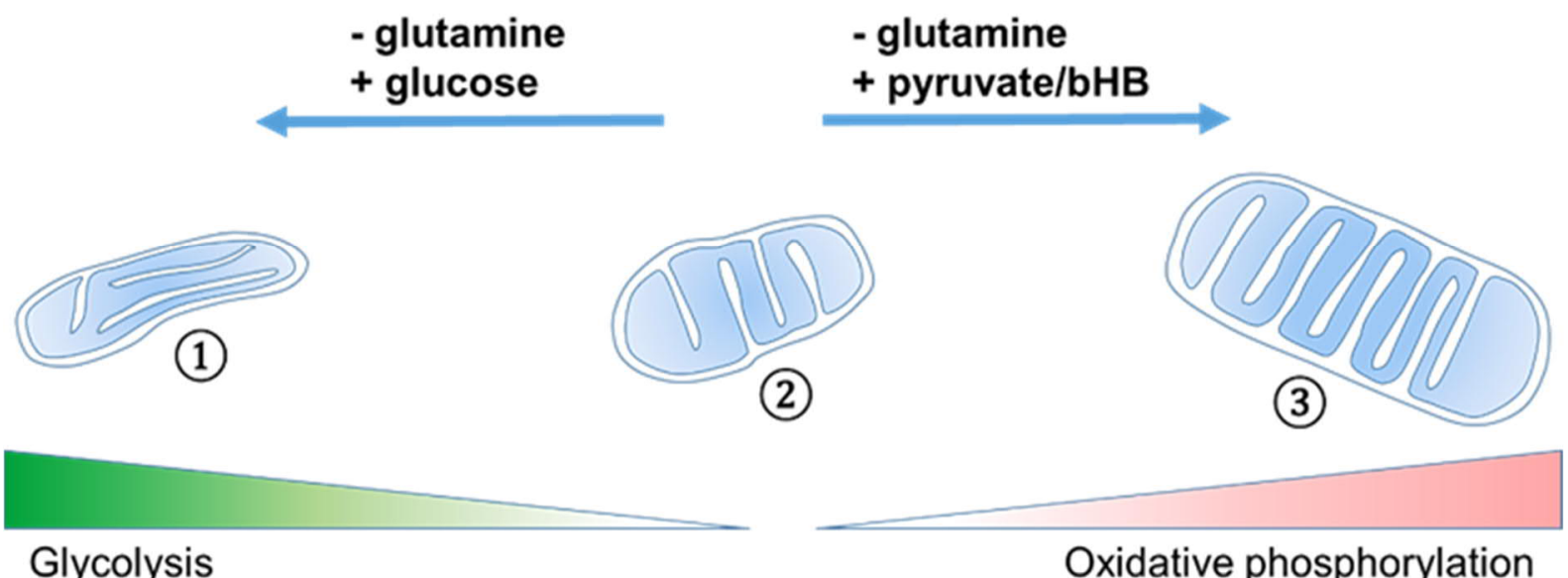

Figure 35: Mitochondrial architecture of cancer cells depending on the available carbon substrate. 1: Cells primarily relying on glycolysis exhibit small mitochondria. The majority of the crista membranes is in parallel to the mitochondrial length axis. 2: Cells cultivated in standard Medium generate ATP via glycolysis and mitochondrial respiration and exhibit mitochondria with orthogonal crista membrane orientation. 3: Cells that can only rely on mitochondrial respiration to generate ATP exhibit large mitochondria with highly abundant crista membranes and orthogonal crista membrane orientation.

In contrast, cells cultivated in medium containing sodium-pyruvate or beta-hydroxybutyrate rely largely on mitochondrial respiration. The higher abundance of crista membranes might indicate an adaptation of the mitochondria to an increased activity of oxidative phosphorylation ( 3 in Figure 35). In order to gain insight into the mechanism underlying the structural changes, western-blot analysis could be used to analyse the regulation of MICOS and the respiratory chain complexes in both metabolic conditions. In combination with oxygen consumption measurements, this could lead to a more complex understanding of the mitochondrial adaptation to the availability of different energy sources. Furthermore, a proteome analysis of all conditions could reveal the precise enzyme regulations during this metabolic change. Beyond that, fluorescence light microscopy will allow to study the overall appearance of the mitochondrial network on a whole cell as well include quantifications of the mitochondrial mass and abundance. Lastly, the metabolic switch could be used as a model system to study crista biogenesis in the future. 


\section{Establishment of a staining protocol for neuronal mitochondria in electron microscopy}

The mitochondrial ultrastructure is challenging to analyse in standard electron microscopy preparations. This is due to an electron-dense staining of the mitochondrial matrix that is obscuring the crista membranes (Figure 36 and Figure 18 A).

In a recent study, Lydia Maus and colleagues immobilized hippocampal brain slices by highpressure freezing (127). After subsequent freeze substitution and resin embedding using 0.1 $\%$ tannic acid and $2 \%$ osmium tetroxide, the mitochondria showed an electron-dense staining of the mitochondrial matrix. Thus, the crista membranes could not be visualized properly (Figure 36 A). Brain slice samples of the hippocampus prepared by David W Freeman and colleagues fixed with aldehydes and stained with $1 \%$ osmium tetroxide and $1 \%$ uranyl acetate as well showed an electron-dense staining of the mitochondrial matrix (199) (Figure 36 B).
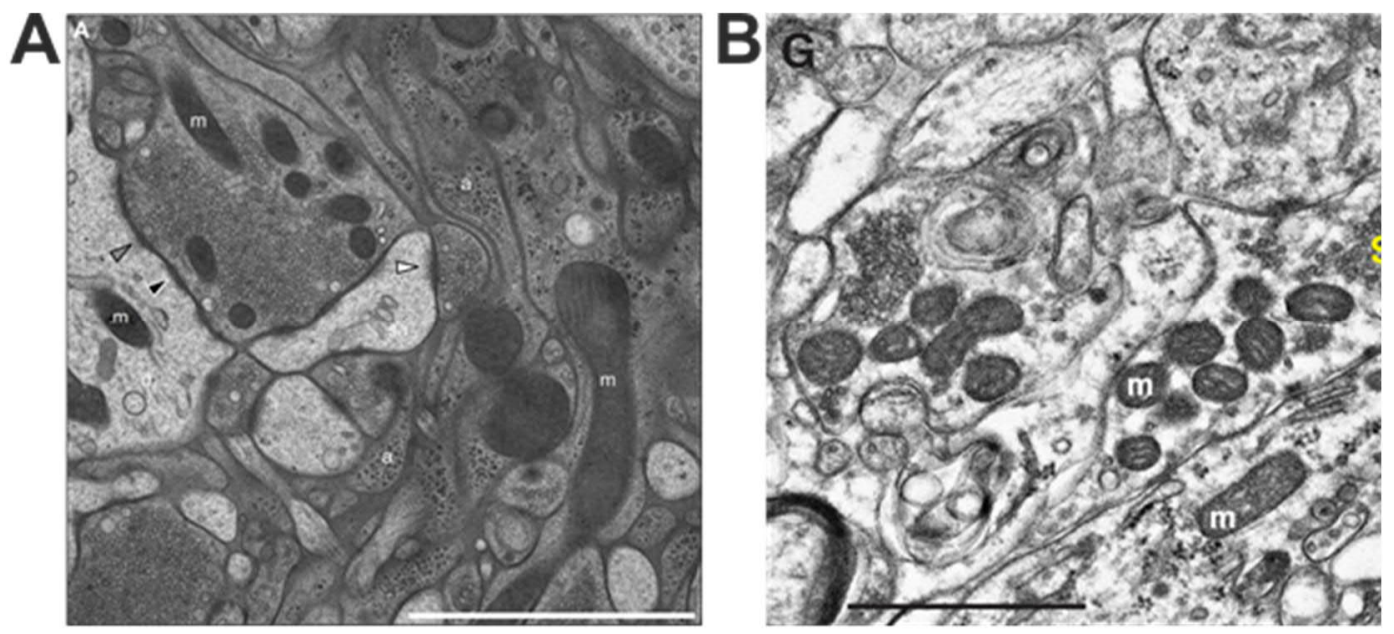

Figure 36: Representative electron micrographs of neuronal mitochondria in hippocampal brain slices. A: Brain slices immobilized by high-pressure freezing and freeze substituted in $0.1 \%$ tannic acid and $2 \%$ osmium tetroxide. Due to the cryo-fixation, the cellular structures are optimally preserved. However, neuronal mitochondria show an electron-dense staining of the matrix and crista membranes are difficult to analyse. Adapted from Figure 1 in (127), mitochondria are labelled with $\boldsymbol{m}$. Scale bar $=2 \mu \mathrm{m}$. B: Brain slices chemically fixed and stained with $1 \%$ osmium tetroxide and $1 \%$ uranyl acetate. Neuronal mitochondria as well show an electron-dense staining of the mitochondrial matrix. Adapted from Figure 1 in (199) mitochondria are labelled with $\mathbf{m}$. Scale bar $=1 \mu \mathrm{m}$.

In TEM preparations of rat hippocampal neurons presented in my study, the same limitations were encountered. The cause for this precipitate and its precise nature could not be identified in my studies. However, I presented protocols to circumvent the staining of the mitochondrial matrix, which allowed for the visualization of cellular details of rat hippocampal neurons in electron microscopy. All protocols aimed to increase the membrane contrast by using additional contrasting agents such as reduced osmium and lead aspartate. The protocols for neuronal staining for TEM presented in this study were a considerable improvement compared to conventional staining protocols. 
Another limitation of 2D cell cultures of rat hippocampal neurons is that they usually exhibit a mitochondrial inner membrane ultrastructure that is less ordered compared to cancer cells and thus, more difficult to interpret (Figure 37 and Figure 19).
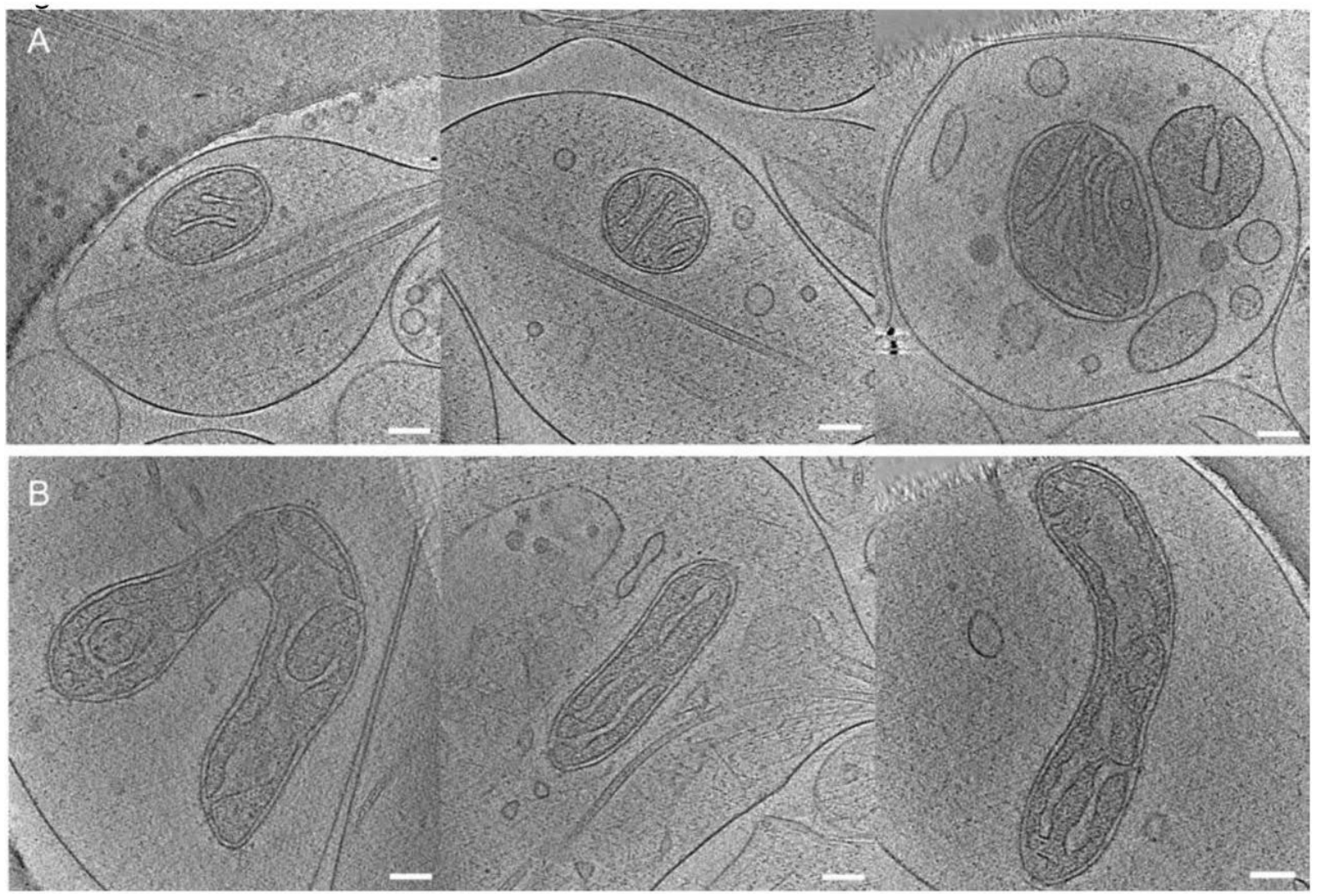

Figure 37: Ultrastructure of axonal mitochondria. Rat hippocampal neurons were plated and grown on Quantifoil grids and after 10 DiV immobilized by plunge freezing in liquid ethane. Cryo-TEM revealed the ultrastructure of axonal mitochondria. The crista membranes are less ordered compared to the mitochondrial ultrastructure of cultured cancer cells and membranes are not always clearly visible. Scale bar $=200 \mathrm{~nm}$. Adapted from Supplementary figure 2 in (131).

Even with the adapted staining protocol presented in this work, the quality of the ultrastructure as well as the staining of the membranes differed largely in different batches of neuronal cells. For a general examination of the samples, a control staining following the standard procedure should always be included and compared in the analysis to examine the overall cell structure appearance.

Future investigations of neuronal mitochondria could benefit from sample immobilization by high-pressure freezing to ensure better preservation of the membrane structures. However, the adapted protocols presented in these studies are in general incompatible with highpressure freezing and freeze substitution. It could be tested, whether chemical prefixation with aldehydes in phosphate buffer would lead to a wash out of mitochondrial matrix components. A subsequent high-pressure freezing fixation of the membranes and staining/embedding at low temperatures may lead to improved contrast of the crista membranes. The method 
CryoCHEM that was recently published might serve as a starting point for the establishment of such a workflow (200).

\section{Mitochondrial occupation of neuronal presynapses}

Mitochondria exhibit a variety of membrane architectures in different cells and organs that is linked to their function (201). In neuronal cells, the structure of mitochondria is significantly different depending on their positioning within the cell body. Several studies showed a significant difference for the size and shape of axonal and presynaptic mitochondria compared to mitochondria localized in the soma or dendrites $(202,203)$. The structural heterogeneity of presynaptic mitochondria points to a functional difference of these organelles. However, the precise role of mitochondria at the neuronal presynapse especially for the supply of ATP is still highly debated.

An electron microscopy study presenting data obtained from cultivated hippocampal brain slices revealed that about $34 \%$ of hippocampal synapses have mitochondria located at the presynapse (108). Stimulation using electrical pulses in a theta burst pattern for 120 minutes did not significantly alter the mitochondrial occupation, suggesting that mitochondria that were already located at the presynapses allowed for sufficient ATP supply (108).

My data is in good agreement with the results demonstrated in that study ( $35 \%$ mitochondrial occupation obtained in resting cultures and stimulated cultures as well). These results open the question for the temporal succession of mitochondrial occupation of presynaptic sites. $A$ decrease in mobility of axonal mitochondria during aging of the neurons might contribute to these observations $(106,204)$. Thus, after initial population of distinctive presynapses, the distribution might not change in the in-vitro models used (2D cell cultures of rat hippocampal neurons and hippocampal brain slices).

In addition, rat hippocampal neurons exhibit large pools of synaptic vesicles after cultivation for 22 DiV. Similar structural details have been described as "phasic" synapses. Phasic synapses are characterised by large pools of synaptic vesicles combined with a lower number of mitochondrial occupation

In contrast to phasic synapses, "tonic" synapses have a higher frequency of mitochondrial occupation in combination with a smaller synaptic vesicle pool (205). In preparations of rat hippocampal neurons, phasic synapses might be more abundant explaining the low numbers of mitochondrial occupied presynapses.

Dynamic processes of mitochondria supporting synaptic activity however cannot be deciphered by using electron microscopy alone. Mitochondrial stalling next to sites of higher 
energy demand might also play a key role for ATP supply at the presynapse (206). These events can be studied using live-cell imaging techniques. Here, fluorescent labels for neuronal mitochondria might be used to determine the overall mobility in neuronal cultures. With the advancement of automated imaging platforms, this could be performed on whole cultures over multiple days. This would solidify our understanding of the mitochondrial adaptation as a whole network (207).

\section{Effects of carbon substrate availability on mitochondrial structure in neuronal cells}

Cultured cancer cells cultivated in presence of different carbon sources were used to classify the structure of mitochondria after a change in the cellular metabolism. Based on that classification, the structure of mitochondria at the presynapse of rat hippocampal neurons resembled a lower mitochondrial activity state under glucose cultivation conditions. Hence, our findings suggest that de novo synthesised ATP required for continuous synaptic activity in neurons might indeed be primarily derived from glycolysis $(56,208)$.

These findings are in contrast to a previous study showing that mitochondria play a critical role in the presynaptic energy supply (102). The study involved FRET-sensors for ATP and suggested that about $30 \%$ of the ATP required for the complete synaptic vesicle turnover (102). A potential reason for the apparent discrepancy might be that these effects were observed for $\sim 20$ minutes in standard medium in contrast to four hours in my conditions during ketosis. Beyond that, the analysis was performed using a different approach and did not include a structural characterisation of the mitochondrial membranes.

Based on the preliminary findings in cancer cell lines, a response and adaptation was expected for rat hippocampal cells when grown on glass coverslips for several days in presence of energy substrates other than glucose (sodium-pyruvate and beta-hydroxybutyrate). Glycolysis should not be a source for ATP production in these cultures and an adaptation of the mitochondrial ultrastructure was expected to adapt in order to compensate for energy demands.

Based on their ultrastructure neuronal mitochondria can be distinguished in three categories (Figure 38). Presynaptic mitochondria of neuronal cells are rather small and the orientation of crista membranes is in parallel to the mitochondrial length axis (1 in Figure 38). The structure of mitochondrial membranes in both the test (ketone bodies) and the control conditions (glucose) were similar. The evaluation of crista membranes resulted in an increased abundance in mitochondria of neuronal cultures cultivated in presence of ketone bodies compared to standard cultivation conditions ( 2 in Figure 38 ). Somatic and dendritic 
mitochondria in both conditions showed large, elongated mitochondria with crista membranes oriented perpendicular to the mitochondrial length axis (3 in Figure 38).

\section{Neuronal mitochondria}

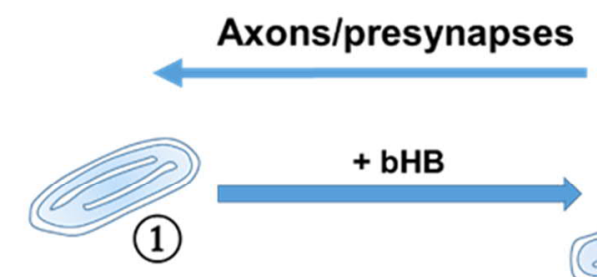

Glycolysis

\section{Soma/dendrites}

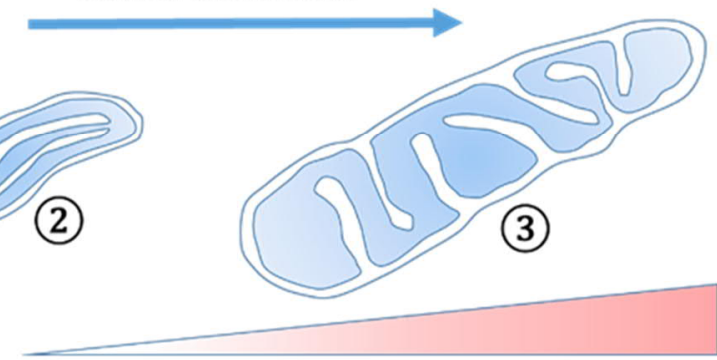

Oxidative phosphorylation

Figure 38: Structural heterogeneity of neuronal mitochondria depending on their positioning in the cells. 1: Axonal and presynaptic mitochondria are short with a small diameter. The crista membranes are oriented in parallel to the mitochondrial length axis. These mitochondria may have a lower OxPhos activity compared to somatic mitochondria. 2: Presynaptic mitochondria of rat hippocampal neurons cultivated in presence of only beta-hydroxybutyrate have an increase of crista membrane abundance but crista membranes are oriented in parallel to the mitochondrial length axis. 3: Mitochondria in the soma and dendrites are elongated and exhibit a larger diameter. The crista membranes are oriented orthogonal to the mitochondrial length axis. These mitochondria may have a higher activity of OxPhos compared to axonal and presynaptic mitochondria.

Several studies reported neuroprotective properties of ketone bodies like beta-hydroxybutyrate when they are utilized by neuronal cells $(183,185,193,197)$. However, to date there are no available studies analysing the influence of ketone bodies on mitochondrial ultrastructure particularly in neuronal cells. In my thesis, I showed the first characterisation of crista membrane architecture of neurons during ketosis. The increased abundance of crista membranes under ketogenic conditions indicated an increased respiratory activity of the mitochondria. The mitochondrial classification obtained in cancer cells (Figure 35) however, might not necessarily reflect neuronal cell classification of mitochondrial activity. For a more pronounced remodelling and reorientation of the crista membranes similar to cultured cancer cells, additional factors might be involved that remained unchanged in presynaptic mitochondria during ketosis. Possible candidates are proteins of the MICOS complex that are encoded in the nucleus, thus the replenishment of these proteins might occur on a longer time scale.

Furthermore, mitochondrial adaptation in neuronal cells might primarily occur on a functional level including changes in the abundance of respiratory chain complex proteins. Therefore, tools are needed to evaluate the precise performance of individual mitochondria and the 
production rate of ATP. A future approach to assess differences in protein levels of complexes in the respiratory chain could include biochemical analysis of synaptosomes such as Western blot analysis and mass spectrometry.

\section{Chemical depolarization of neuronal cells}

Chemical depolarization of neuronal cells with high concentrations of $\mathrm{KCl}$ is a well-established method to evoke synaptic activity. Chemical depolarization does not represent a fully physiological condition (209), however it has been shown to cause similar cellular responses in cultured neuronal cells comparable to electrical stimulation (210-213). This includes a functional exo- and endocytic cycle of synaptic vesicles responses as well as cellular adaptations on a gene level and protein phosphorylation.

An advantage of chemical depolarization is that it affects nearly all neuronal cells in the culture. Electrical stimulation might only affect a subset of cells making an electron microscopy base study more challenging because synapses cannot be distinguished based on their activity in the electron micrographs $(209,214)$.

Hence, in this thesis, the depolarization with high concentrations of $\mathrm{KCl}$ was used. The use of the same culture medium turned out to be necessary because cultures suffered from intense stress leading to cell death of the entire culture when fresh medium was used. The toxic effect of fresh Neurobasal-medium addition affected cultures after synaptogenesis (around $14 \mathrm{DiV}$ ). This might be caused by the relatively high concentrations of cysteine in the Neurobasal-A formulation ( $260 \mu \mathrm{M})$ which might cause excitotoxicity in a NMDA dependent manner (215). Toxic effects were not observed when using the supernatant of the original culture medium of the neuronal cultures and adding $\mathrm{KCl}$. This was an important finding to enable the study of long-term effects of stimulated synaptic activity in cultured neurons.

Using these depolarization conditions, my data show a significant increase of crista membrane abundance ( 20\%) compared to non-depolarized cells. This increase might represent a mitochondrial adaptation, which facilitates sufficient ATP supply during prolonged synaptic activity in neuronal cultures. Prolonged stimulation did not lead to an alteration of the overall membrane architecture and the crista membrane orientation, which would be expected in a state of high presynaptic energy demand based on the classification model of cancer cells. However, as mentioned earlier, the mechanism of mitochondrial adaptation in cancer cells might include factors beyond the switch to oxidative phosphorylation and thus, not be entirely representative for mitochondrial adaptation in synapses. Structural adaptations of mitochondria to electrical long-term stimulation have been previously shown in hippocampal brain slices (108). In this study, Smith et al. demonstrated a change in mitochondrial membrane 
architecture, however, the classification system differs to the system in the present work is thus challenging to put into context. Furthermore, changes in the abundance of crista membranes were not analysed in this study and thus, cannot be directly compared to the findings in the present work. To exclude artefacts caused by chemical fixation and ethanol dehydration, these effects should be compared to brain slices prepared by cryo-fixation.

The relatively mild structural changes in neurons compared to cancer cells might also be attributed to an overall higher abundance of mitochondria at the presynapse under prolonged activity $(104,206,216)$, which serve the energy needs for synaptic transmission. To test this hypothesis, the mitochondrial abundance close to presynaptic sites before and after stimulation should be quantified in neuronal cells in ketosis. This can be achieved by serial section transmission electron microscopy of individual presynapses. However, due to the increased technical efforts this methodology entails, a light microscopy approach with labelled mitochondria might be more straightforward. There, the change on mitochondrial occupation could be evaluated with fluorescently labelled mitochondria together with presynaptic markers (for example vGLUT1 $(217,218)$ ).

Lastly, to be able to correlate the stimulation-evoked structural changes in mitochondria to a response on a functional level approaches such as quantitative western blotting need to be pursued. To this end, both, whole cells and synaptosomes could be tested for the proteins necessary for ATP generation including the respiratory chain complexes $(219,220)$. Furthermore, levels of key proteins of glycolysis could be tested in a similar way. However, I found that there is a high variability of cells of different batches, which will make a detailed and reproducible analysis via western blot challenging. The overall mitochondrial mass and abundance could be further analysed by using for example, fluorescence light microscopy $(221,222)$. This data could be completed with additional oxygen consumption assays $(223$, 224).

\section{Mitochondrial adaptation in presynapses of the cochlear nucleus in mice}

3D imaging using electron microscopy is applied to understand the structural inter- and intracellular organization of the brain $(134,225,226)$. Recently, SBF-SEM was employed to study mitochondria in the calyx of Held in mice in different stages of development (111). There, the authors found an increase of the bouton volume of calyces during development of the brain area where they compared P7 animals (immature calyces) to P21 animals (matured calyces). Further, they reported an increase of the mitochondrial volume in the calyx of Held as well as minor terminals of calyxes during ageing of the animals. This increase of volume indicates an adaptation of mitochondria during the development of a high-fidelity synapse. 
In my thesis, I analysed, whether a similar mitochondrial adaptation occurs on smaller and presumably less specialized presynapses that are localized in the cochlear nucleus (Figure 39). This region of the brain was chosen because of the onset of hearing in rodents at 12 days (P12, 1 in Figure 39) $(227,228)$, thus with the onset of hearing, an increase of synaptic activity is to be expected. In my thesis, I presented adaptations of the presynaptic structure in an animal at P20 that were in good agreement to the observed changes in matured calyxes (111).

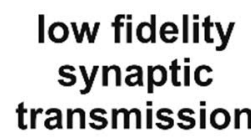

Energetic demand

Presynaptic

mitochondria
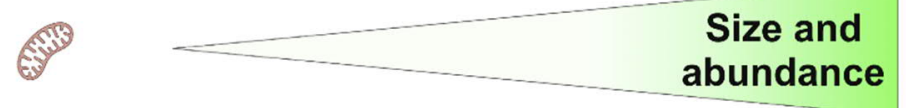

abundance

\section{transmission}

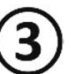

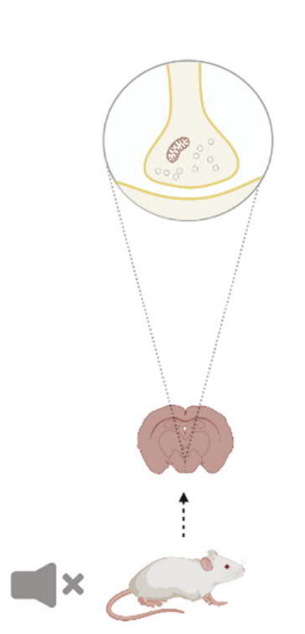

P9

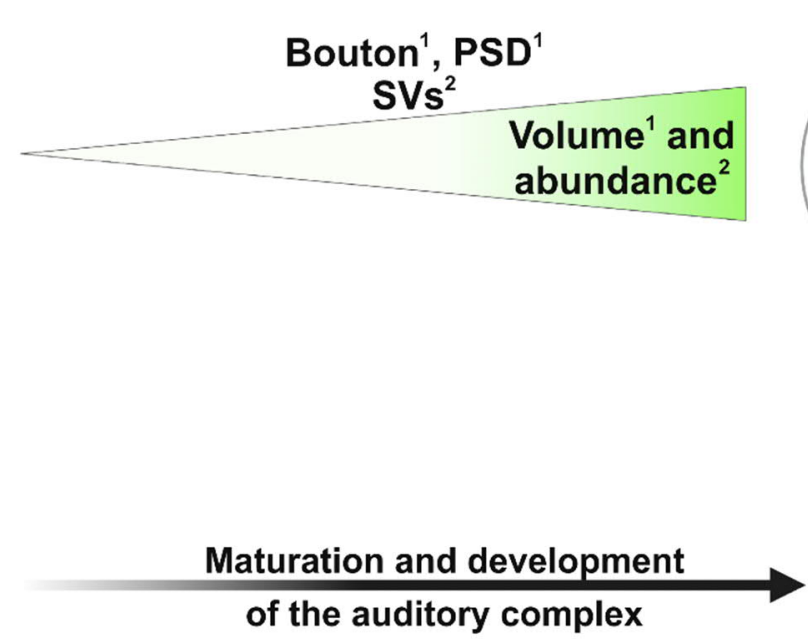

of the auditory complex

Figure 39: Structural adaptations of presynapses in the cochlear nucleus after maturation and development. 1: With development and maturation of the auditory complex, the onset of hearing occurs at P12 in mice. 2: The structure of presynapses in the cochlear nucleus adapts to high-fidelity signal transmission after the onset of hearing. These adaptations include an increase in bouton size and PSD size and as well as an increased abundance of synaptic vesicles. 3: The abundance of mitochondria and the mitochondrial size is significantly increased in presynapses of the cochlear nucleus after the onset of hearing. 4: The observation of structural changes during development of the cochlear nucleus indicate an increased energy demand for presynapses that is met by the structural adaptation of the presynapses and in particular of presynaptic mitochondria. The figure was created with BioRender.com.

The cochlear nucleus is a central part in the signal transmission of the auditory complex. This brain area might have a higher activity of the synapses as compared to the hippocampus that was reflected by a higher abundance of mitochondria at the presynapses at P9. More importantly, the abundance increased during ageing in the P20 animal, where I found about 
$80 \%$ of all presynapses to have an occupation with mitochondria. This demonstrated a major adaptation of the entire mitochondrial network that was not seen in in-vitro models $(106,108$, $204,229)$ and in my own analyses of rat hippocampal neurons presented in this thesis.

A detailed analysis of randomly selected presynaptic boutons revealed further differences. First, it was shown that the occupation with mitochondria is accompanied with a higher abundance of synaptic vesicles in the same boutons. The size of the PSD correlates with presynaptic activity $(230,231)$. The sizes of the corresponding post-synaptic density (PSD) of synapses with presynaptic mitochondria at P9 were increased as well compared to synapses without presynaptic mitochondria. The size of the synaptic vesicle pools was further increased in the P20 animal, which suggest the establishment of stable and highly active connections with the onset of hearing $(111,232)$. Further, the PSD-size of synapses in the P20 animal as well showed an increase ( 2 in Figure 39). In addition to these general comparisons of the presynaptic boutons, I found that the volume of presynaptic mitochondria strongly increased in the P20 animal compared to the P9 animal (3 in Figure 39). All observations of structural changes from P9 to P20 suggested an increase in energy demand of presynapses in the cochlear nucleus that caused an adaptation of the presynaptic structure and in particular of the presynaptic mitochondria (4 in Figure 39).

In conclusion, the age-dependent development of highly active presynapses caused a mitochondrial adaptation in presynapses of the cochlear nucleus in mice. For future studies, a combination with transmission electron tomography or scanning transmission electron microscopy could be conducted in the same brain area. With the higher lateral resolution of electron tomography, extended analysis of the active zone could be included to the existing data set (127). This could include the precise distribution of synaptic vesicles as well as the fraction of docked vesicles to the active zone. It would be furthermore interesting to expand these analyses on different brain areas as well. The cochlear nucleus is central for the transmission of signals within the auditory complex. Therefore, individual synapses might be continuously more active than, for example, in the hippocampus. This is suggested with the already higher mitochondrial occupation of the presynapses at the age of 9 days. In additional experiments, areas of the hippocampus could be tested and compared. Further, the visual cortex in rodents as well is developed at around 12 days and should be analysed in comparison (233).

In summary, section 1 of this thesis presented an extensive electron microscopy based analysis of mitochondria. In cancer cells, an extensive mitochondrial adaptation during metabolic changes was demonstrated. To date, this was the first analysis showing a complex crista membrane remodelling indicating a defined mitochondrial architecture for either inactive 
or highly active mitochondria. In future experiments, the regulation of protein complexes in MICOS and the respiratory chain could be analysed in more detail using western blot analysis and mass spectrometry assisted proteomics. Then, the transition of mitochondria with low activity to a state of high activity could be induced using the protocols established in this study. Cultivated rat hippocampal neurons are widely used to study mechanistic aspects of synaptic function. However, to analyse the mitochondrial adaptation in presynapses, this model system faces limitations. A better preservation of the membranous ultrastructure could enable a more precise analysis of crista membranes in presynaptic mitochondria. This could be enabled with adapted staining protocols of high-pressure frozen neurons and may be extended to cryoelectron tomography of plunge frozen cells. Furthermore, the analysis could be extended with the correlative protocols presented in section 2. There, a precise correlation of synaptic activity to mitochondrial structure and protein turnover of highly active presynapses could be achieved.

Finally, the in-vivo analysis of presynapses could be expanded with imaging of other brain areas. Extensive data of minor presynapses and presynaptic mitochondria is needed to understand the precise mitochondrial adaptation during development and synaptic activity. Therefore, animals could also be fed with a ketogenic diet and the protocols presented here for in-vitro neurons applied to analyse this metabolic adaptation of mitochondria in-vivo. 


\section{Section 2 - An approach to correlative imaging}

Section 2 presented the establishment of protocols for correlative imaging of biological samples. In a first step the design and implementation of a CLEM-suitable substrate was demonstrated. This allowed a robust and straightforward application of live cell light microscopy with the subsequent structural analysis via electron microscopy. In this work, the successful protocol establishment was demonstrated using Bax-EGFP transfected cells as a model system. There, the feasibility of the protocol was shown including a detailed ultrastructural analysis of mitochondria during intrinsic apoptosis. Next, these protocols were expanded to allow the high-accuracy localization of functional information within the corresponding ultrastructure. I presented a workflow using HeLa cells utilizing a fluorescent label for mitochondrial membrane potential as a suitable sample for the protocol establishment. This approach was then expanded to a correlation of high-accuracy CLEM with nanoscale secondary ion mass spectrometry (NanoSIMS). It was demonstrated that this extended correlative approach could extract information from an embedded biological sample that was hitherto inaccessible with conventional protocols for correlative imaging. In the following, a detailed discussion of all subsections is presented.

\section{Design of CLEM-compatible cell substrates}

Correlative light and electron microscopy (CLEM) can deliver comprehensive data on functional aspects in the structural context of cells and tissues. One key aspect to the successful application of CLEM is the retrieval of regions of interest first imaged by light microscopy in the electron micrographs.

At the beginning of this study, various commercial solutions already existed to allow successful correlation. These solutions include plastic substrates where a finder grid is imprinted which is visible during light microscopy investigations with a subsequent transfer of the finder grid to the electron microscopy resin. These plastic substrates are usually compatible with standard fluorescence light microscopy approaches such as epifluorescence imaging but already fall short when used on a confocal microscope. This is because these plastic substrates exhibit a poor point spread function of the signals obtained from the sample and a high extent of autofluorescence (234).

A more advanced approach is the etching of a reference map into glass cover slides allowing better use during fluorescence light microscopy investigations (for example Mattek). This reference map is transferred as a 3D-print on the final resin block in order to retrieve the area of interest. The advantage is that cells can be imaged through the coverslip resulting in a short distance from objective to the signal itself and finally to a precise recording of the signals. The 
coverslips are then detached from the resin block by freeze thawing with the use of liquid nitrogen and boiling water in order to separate the two materials. In practice, this can lead to the breaking of the resin block and/or the reference map on the resin block. Additionally, the interface of the resin and the embedded cells often suffers from cracks introduced by the procedure. This leads to a low-quality sample for sectioning and electron microscopy.

Another strategy is to dissolve the glass coverslip in low concentrations of fluoric acid ( 5\%) over a longer period. Because fluoric acid also penetrates into the resin, this also results in resoftening of the resin within a couple of nanometres of the sample and additional washout of staining reagents (such as en-bloc staining of lead aspartate). This might be because precipitates of lead and uranyl organophosphates are susceptible to low $\mathrm{pH}$ conditions and might be re-solubilized.

The size of the reference grid in these commercial substrates ranges usually from $50 \mu \mathrm{m} \times 50$ $\mu \mathrm{m}$ up to $600 \mu \mathrm{m} \times 600 \mu \mathrm{m}$. While the first can obtain a single cell within one labelled box, the sample is usually very prone to damage during the detachment of the glass coverslip. On the other hand, the larger sizes of $500 \mu \mathrm{m} \times 500 \mu \mathrm{m}$ and $600 \mu \mathrm{m} \times 600 \mu \mathrm{m}$ make it more time consuming to retrieve the area of interest on the ultra-thin sections.

Therefore, a robust in-house protocol to produce reference substrates was implemented. By using commercial TEM finder grids as a template and the subsequent carbon-evaporation, a reference map is created on any desired cell substrate. Substrates can be produced in large numbers at a low cost and can be individualized for the specific needs (adaptation of different finder girds or alternatively using a customized mask). By using TEM finder grids, indexed regions of the gird are about $100 \mu \mathrm{m} \times 100 \mu \mathrm{m}$ in size, which was optimal for adherent cells. It was shown that the reference map is visible in fluorescence light microscopy when at least $\sim 10 \mathrm{~nm}$ of carbon are deposited on the substrate and the reference map is transferred completely without damage to the final resin blocks.

A similar substrate was created by carbon sputter coating of a replica on sapphire discs that is now commercially available (235). However, by using sapphire discs as a cell substrate these CLEM substrates are substantially more expensive than the approach I presented in this thesis. Further, the sample processing following chemical immobilization of the cells does not require sapphire cell substrates.

\section{Implementation of live cell CLEM}

In order to establish the workflow for correlative light and electron microscopy, HeLa-cells expressing Bax-EGFP were analysed in various stages of apoptosis as a model system. 
A hallmark of intrinsic apoptosis is the rupture of the mitochondrial outer membrane that leads to large lipidic pores up to the size of several hundred nanometres (236). The formation of these lipidic pores after the activation of the pro-apoptotic protein Bax was reported in a recent study of Kate McArthur and colleagues (237). However, in their study they focused on the release of mitochondrial DNA during intrinsic apoptosis and not the mitochondrial membrane structure and remodelling.

Recently, Nicholas R Ader and colleagues reported Bax-localizations to the mitochondrial membrane using cryo-correlative light and electron microscopy (238). They demonstrated mitochondrial outer membrane ruptures in electron tomography that co-localized with the signal localization of Bax-EGFP in light microscopy. Additionally, they were able to prove that the large ruptures of the mitochondrial outer membrane were not an artefact caused by the slower immobilization of the cells during classical aldehyde fixation since their samples were cryo-immobilized. However, in the cryo-immobilized samples of cell pellets, the mitochondria could not be analysed completely because the polarity of the mitochondrial network is lost after detachment and pelleting of the cells prior to freezing.

In my thesis, I established 3D-CLEM by using the self-made CLEM substrates by carbon sputtering on Aclar foil. With this system, I showed a spatial localization of Bax-EGFP in light microscopy that co-localizes with mitochondria showing membrane ruptures as well as a proteinaceous density in the electron micrographs. These observations are in good agreement with the studies of Ader and colleagues.

In my work, it was demonstrated that live-cell light microscopy correlated to electron microscopy could serve as a tool to analyse multiple cells in a single sample. This helped to understand temporal succession by being able to observe and distinguish different time-points. This was demonstrated in HeLa cells at different stages of apoptosis (Figure 40). Especially changes of the mitochondrial membranes in early apoptosis would be difficult to study in cells that are processed as a pellet. The data presented in my thesis clearly demonstrated the advantages of correlative imaging in HeLa cells using live cell fluorescence microscopy and TEM to investigate the cellular structure at different concentrations and time scales of a transfected protein of interest. 


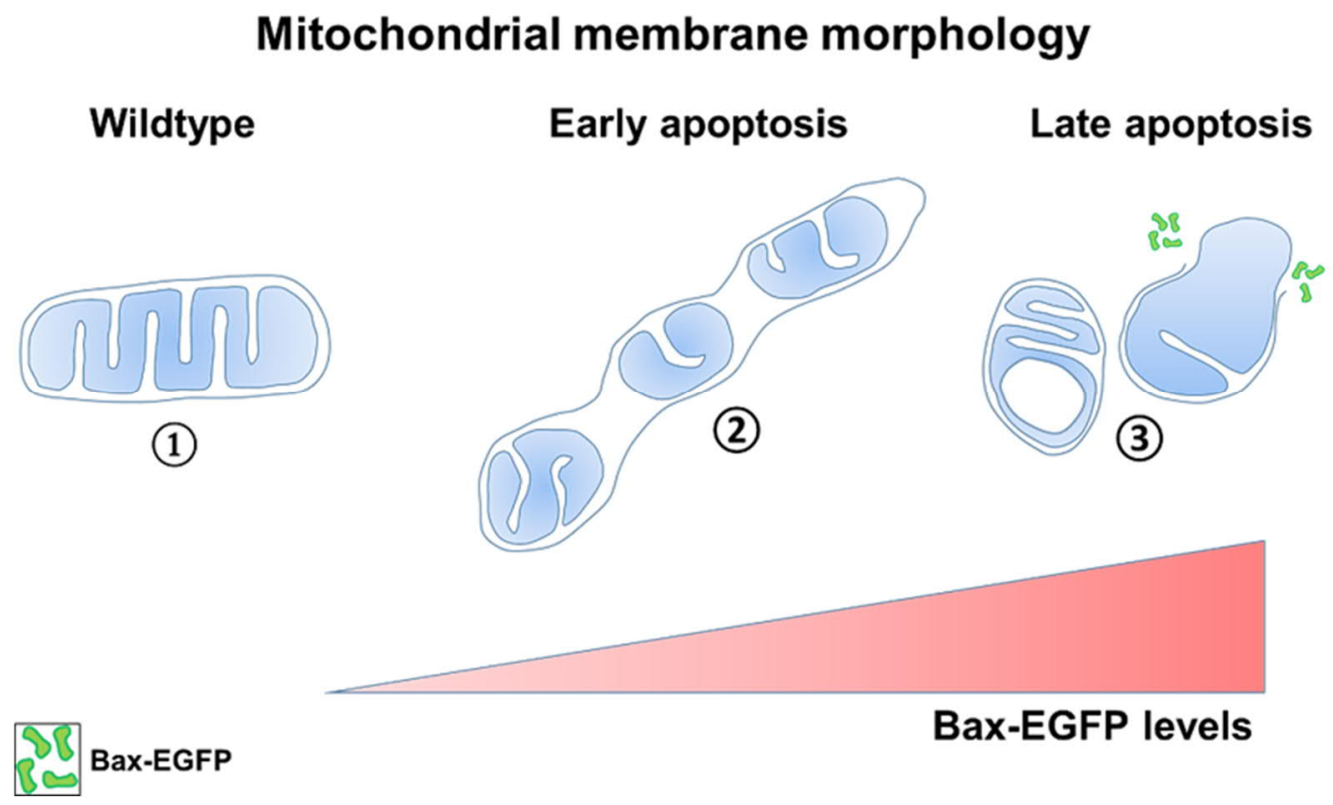

Figure 40: Mitochondrial membrane morphology in HeLa cells during intrinsic apoptosis. 1: Mitochondria of cells without Bax-EGFP expression exhibit a tubular mitochondrial network with ordered stacks of crista membranes. 2: Mitochondria of cells with low expressions of BaxEGFP show mitochondrial matrix spaces that separate in the outer membrane tube. 3: Mitochondria of cells with high expression levels of Bax-EGFP show aberrant and fragmented mitochondria. A subset of mitochondria show outer membrane ruptures in close proximity to Bax.

For the future application of the live-cell CLEM approach on neuronal cultures, fluorescent markers for synaptic activity in combination with markers for mitochondria could be used to pinpoint specifically mitochondria in active presynapses for a structural characterisation.

\section{Implementation of high-accuracy CLEM}

To overcome challenges of successful data registration in correlative imaging, high-accuracy CLEM can be employed. Here, the data of both imaging modalities is obtained postembedment and thus, signals for fluorescence light microscopy are recorded on the final thinsections as usually prepared for transmission electron microscopy (169, 239). In addition, labelling of proteins with antibodies on thin-sections could be used (240). Specific labelling with antibodies was demonstrated on either Tokuyasu-cryo sections or Lowicryl sections (171, 241). The use of primary antibodies is preferred to detect endogenous levels of protein. However, sparse labelling as well as extensive optimization for every antibody used makes these approaches less efficient and easy to use (242).

I demonstrated the compatibility of different labelling approaches including fusion proteins of fluorescent proteins as well as chemical dyes and affinity probes for correlative imaging of HeLa cells. Using chemical dyes or fluorescent proteins, the functional information obtained in 
light microscopy could be located in the electron micrographs with a localization error smaller than $50 \mathrm{~nm}$. This of course is an artificial value as the recording of the fluorescence signal was diffraction limited in epifluorescence microscopy. The localization precision could be improved using advanced light microscopy techniques such as STED nanoscopy, STORM or GSDIM $(240,243)$. However, I found that longer incubation times of the thin-sections result in significant deterioration of the cellular structures, which has to be considered for the use of any advanced light microscopy imaging for CLEM.

Compared to the live-cell CLEM approach I presented in this thesis, my established highaccuracy CLEM protocol ensured the superior structural preservation due to the immobilization by means of high-pressure freezing, thus minimizing structural perturbations in the sample (170). High-accuracy CLEM combines excellent structural preservation with a high number of cells that can be analysed since large sections of a cell pellet can be investigated in a short time on a single sample grid. In addition, resin-based approaches would allow for electron tomography of $300 \mathrm{~nm}$ thick sections as well as serial sectioning preparations in 2D and 3D.

\section{Expanding the information obtained by correlative imaging}

To date, a correlation of NanoSIMS to other imaging modalities is needed in order to distinguish features of interest. Within this study, I presented an approach to combine the benefits of all three imaging modalities on one resin embedded sample to allow the extraction of information that is not accessible with conventional correlative approaches. Precisely, highaccuracy CLEM was used to extract functional and structural information and the registered images served as a map to guide subsequent metabolic imaging. This protocol is straightforward because the sample preparation is compatible with all three techniques as demonstrated in this thesis.

The feasibility of the protocol was shown by using HeLa cells labelled with Mitotracker Deep Red FM to stain mitochondria. After successful sample preparation and an additional DAPI staining of the thin section, the structure of the nucleus as well as the position of individual mitochondria was recorded in an epifluorescence microscope. Subsequent transmission electron microscopy revealed the underlying cellular structure. A final recording of the ${ }^{12} \mathrm{C}_{2}$, ${ }^{12} \mathrm{C}^{13} \mathrm{C}^{-},{ }^{12} \mathrm{C}^{14} \mathrm{~N}^{-},{ }^{12} \mathrm{C}^{15} \mathrm{~N}^{-},{ }^{31} \mathrm{P}^{-}$and ${ }^{32} \mathrm{~S}^{-}$ions in NanoSIMS revealed the chemical composition of the cells showing fundamental differences in the elemental distribution across different cell sites and organelles (145).

In the past, this correlation was achieved with either using light microscopy $(244,245)$ (or even super resolution light microscopy (246)) or electron microscopy (247-249). 
An extensive analysis can further lead to a better understanding of how organelles appear in NanoSIMS measurements. An additional benefit of the presented approach is the superior structural preservation of the cells by means of high-pressure freezing. This not only allows for a general localization of elemental signals but also enables the detailed analysis of membranous structures. Expanding the protocols on neuronal cells will allow the multidimensional extraction of information on presynaptic structures. This correlative approach will help to understand the complex nature of synaptic activity. Here, the activity of neuronal sites can be brought into context with the overall turnover of different sites and organelles.

In summary, section 2 of this work presented multiple strategies for correlative imaging of cells. Cells of interest can be identified in live-cell light microscopy and analysed with high detail in transmission electron microscopy. 3D CLEM can be applied to understand the function of a target protein in the structural context. High-accuracy CLEM can then be used to understand function-structure relationships in more detail because the data is correlated precisely in all three dimensions. An additional correlation of high-accuracy CLEM to metabolic imaging can obtain comprehensive information about protein localizations, structure and chemical composition of sub-cellular areas. The protocols presented in this work could be applied on cultured rat hippocampal neurons in-vitro and to neuronal cells in rats or mice in-vivo. Live cell recordings with recently developed fluorescent reporters for synaptic activity such as sensitive calcium-sensing fusion proteins would highlight presynapses with high activity. A subsequent correlation to room temperature transmission electron microscopy with NanoSIMS could highlight functional differences of neuronal presynapses that are not accessible when using electron microscopy alone. This work presented a promising workflow that can be expanded with the use of stable isotope labelling of proteins and DNA to measure the turnover of presynaptic components in a pulse chasing approach. In combination with live cell CLEM, a detailed comparison of protein and DNA turnover of presynaptic mitochondria can be correlated to the activity of these sites. Furthermore, the in-vivo analysis presented in this work could also be expanded by feeding the animals stable isotope enriched food. The same data could then be obtained in a physiological relevant environment of the neurons. In addition, it would be interesting to see a correlation of cryo-CLEM with subsequent freeze-drying and correlation to NanoSIMS. 


\section{References}

1. A. L. Hodgkin, A. F. Huxley, A quantitative description of membrane current and its application to conduction and excitation in nerve. J Physiol 117, 500-544 (1952).

2. J. Del Castillo, B. Katz, Quantal components of the end-plate potential. J Physiol 124, 560-573 (1954).

3. P. Fatt, B. Katz, Spontaneous Subthreshold Activity at Motor Nerve Endings. J PhysiolLondon 117, 109-128 (1952).

4. Y. Zhou, N. C. Danbolt, Glutamate as a neurotransmitter in the healthy brain. J Neural Transm (Vienna) 121, 799-817 (2014).

5. N. G. Bowery, T. G. Smart, GABA and glycine as neurotransmitters: a brief history. $\mathrm{Br}$ J Pharmacol 147 Suppl 1, S109-119 (2006).

6. M. Berger, J. A. Gray, B. L. Roth, The expanded biology of serotonin. Annu Rev Med 60, 355-366 (2009).

7. N. Chenouard, F. Xuan, R. W. Tsien, Synaptic vesicle traffic is supported by transient actin filaments and regulated by PKA and NO. Nat Commun 11, 5318 (2020).

8. A. A. Cole, X. Chen, T. S. Reese, A Network of Three Types of Filaments Organizes Synaptic Vesicles for Storage, Mobilization, and Docking. J Neurosci 36, 3222-3230 (2016).

9. T. Schikorski, C. F. Stevens, Quantitative Ultrastructural Analysis of Hippocampal Excitatory Synapses. The Journal of Neuroscience 17, 5858-5867 (1997).

10. J. D. Clements, R. A. Lester, G. Tong, C. E. Jahr, G. L. Westbrook, The time course of glutamate in the synaptic cleft. Science 258, 1498-1501 (1992).

11. S. E. Ahmari, S. J. Smith, Knowing a Nascent Synapse When You See It. Neuron 34, 333-336 (2002).

12. N. Scheefhals, H. D. MacGillavry, Functional organization of postsynaptic glutamate receptors. Mol Cell Neurosci 91, 82-94 (2018).

13. Y. T. Liu, C. L. Tao, P. M. Lau, Z. H. Zhou, G. Q. Bi, Postsynaptic protein organization revealed by electron microscopy. Curr Opin Struct Biol 54, 152-160 (2019).

14. T. Suzuki, R. Tanaka, Postsynaptic density visualized by whole mount electron microscopy. Neuroscience Research 3, 226-236 (1986).

15. R. Jahn, D. Fasshauer, Molecular machines governing exocytosis of synaptic vesicles. Nature 490, 201-207 (2012).

16. G. Pigino, G. A. Morfini, S. T. Brady, in Basic Neurochemistry (Eighth Edition), S. T. Brady, G. J. Siegel, R. W. Albers, D. L. Price, Eds. (Academic Press, New York, 2012), pp. 119-145. 
17. M. Yoshihara, J. T. Littleton, Synaptotagmin I Functions as a Calcium Sensor to Synchronize Neurotransmitter Release. Neuron 36, 897-908 (2002).

18. N. A. Courtney, H. Bao, J. S. Briguglio, E. R. Chapman, Synaptotagmin 1 clamps synaptic vesicle fusion in mammalian neurons independent of complexin. Nat Commun 10, 4076 (2019).

19. W. A. Catterall, Structure and regulation of voltage-gated Ca2+ channels. Annu Rev Cell Dev Biol 16, 521-555 (2000).

20. K. Dunlap, J. I. Luebke, T. J. Turner, Exocytotic Ca2+ channels in mammalian central neurons. Trends Neurosci 18, 89-98 (1995).

21. T. P. Snutch, P. B. Reiner, Ca2+ channels: diversity of form and function. Current Opinion in Neurobiology 2, 247-253 (1992).

22. H. W. Tedford, G. W. Zamponi, Direct G protein modulation of Cav2 calcium channels. Pharmacol Rev 58, 837-862 (2006).

23. B. Ceccarelli, W. P. Hurlbut, A. Mauro, Turnover of transmitter and synaptic vesicles at the frog neuromuscular junction. J Cell Biol 57, 499-524 (1973).

24. J. E. Heuser, T. S. Reese, Evidence for recycling of synaptic vesicle membrane during transmitter release at the frog neuromuscular junction. J Cell Biol 57, 315-344 (1973).

25. Z. Farsi et al., Clathrin coat controls synaptic vesicle acidification by blocking vacuolar ATPase activity. Elife 7, (2018).

26. J. R. Morgan, H. S. Comstra, M. Cohen, V. Faundez, Presynaptic membrane retrieval and endosome biology: defining molecularly heterogeneous synaptic vesicles. Cold Spring Harb Perspect Biol 5, a016915 (2013).

27. Q. Gan, S. Watanabe, Synaptic Vesicle Endocytosis in Different Model Systems. Front Cell Neurosci 12, 171 (2018).

28. M. E. Maxson, S. Grinstein, The vacuolar-type $\mathrm{H}(+)$-ATPase at a glance - more than a proton pump. J Cell Sci 127, 4987-4993 (2014).

29. M. E. Finbow, M. A. Harrison, The vacuolar H+-ATPase: a universal proton pump of eukaryotes. Biochem J 324 ( Pt 3), 697-712 (1997).

30. S. Gowrisankaran, I. Milosevic, Regulation of synaptic vesicle acidification at the neuronal synapse. lubmb Life 72, 568-576 (2020).

31. R. D. Blakely, R. H. Edwards, Vesicular and plasma membrane transporters for neurotransmitters. Cold Spring Harb Perspect Biol 4, (2012).

32. Z. Farsi, R. Jahn, A. Woehler, Proton electrochemical gradient: Driving and regulating neurotransmitter uptake. Bioessays 39, (2017).

33. C. Pulido, T. A. Ryan, Synaptic vesicle pools are a major hidden resting metabolic burden of nerve terminals. bioRxiv, 2020.2011.2016.385575 (2020). 
34. P. Chi, P. Greengard, T. A. Ryan, Synapsin dispersion and reclustering during synaptic activity. Nat Neurosci 4, 1187-1193 (2001).

35. N. J. Pavlos, R. Jahn, Distinct yet overlapping roles of Rab GTPases on synaptic vesicles. Small GTPases 2, 77-81 (2011).

36. S. Giovedi et al., Synapsin is a novel Rab3 effector protein on small synaptic vesicles. I. Identification and characterization of the synapsin I-Rab3 interactions in vitro and in intact nerve terminals. J Biol Chem 279, 43760-43768 (2004).

37. J. J. Harris, R. Jolivet, D. Attwell, Synaptic energy use and supply. Neuron 75, 762-777 (2012).

38. M. J. Devine, J. T. Kittler, Mitochondria at the neuronal presynapse in health and disease. Nat Rev Neurosci 19, 63-80 (2018).

39. I. A. Simpson et al., The facilitative glucose transporter GLUT3: 20 years of distinction. Am J Physiol Endocrinol Metab 295, E242-253 (2008).

40. J. M. Ferreira, A. L. Burnett, G. A. Rameau, Activity-dependent regulation of surface glucose transporter-3. J Neurosci 31, 1991-1999 (2011).

41. P. Weisova, C. G. Concannon, M. Devocelle, J. H. Prehn, M. W. Ward, Regulation of glucose transporter 3 surface expression by the AMP-activated protein kinase mediates tolerance to glutamate excitation in neurons. J Neurosci 29, 2997-3008 (2009).

42. G. Ashrafi, Z. Wu, R. J. Farrell, T. A. Ryan, GLUT4 Mobilization Supports Energetic Demands of Active Synapses. Neuron 93, 606-615 e603 (2017).

43. J. Pearson-Leary, E. C. McNay, Novel Roles for the Insulin-Regulated Glucose Transporter-4 in Hippocampally Dependent Memory. J Neurosci 36, 11851-11864 (2016).

44. S. J. Vannucci et al., GLUT4 glucose transporter expression in rodent brain: effect of diabetes. Brain Research 797, 1-11 (1998).

45. M. Kobayashi, H. Nikami, M. Morimatsu, M. Saito, Expression and localization of insulin-regulatable glucose transporter (GLUT4) in rat brain. Neurosci Lett 213, 103106 (1996).

46. D. Attwell, S. B. Laughlin, An energy budget for signaling in the grey matter of the brain. J Cereb Blood Flow Metab 21, 1133-1145 (2001).

47. M. J. Hubley, B. R. Locke, T. S. Moerland, The effects of temperature, $\mathrm{pH}$, and magnesium on the diffusion coefficient of ATP in solutions of physiological ionic strength. Biochimica et Biophysica Acta (BBA) - General Subjects 1291, 115-121 (1996).

48. C. Werner, T. Doenst, M. Schwarzer, in The Scientist's Guide to Cardiac Metabolism, M. Schwarzer, T. Doenst, Eds. (Academic Press, Boston, 2016), pp. 39-55. 
49. A. Kumari, in Sweet Biochemistry, A. Kumari, Ed. (Academic Press, 2018), pp. 1-5.

50. R. A. Bender, in Brenner's Encyclopedia of Genetics (Second Edition), S. Maloy, K. Hughes, Eds. (Academic Press, San Diego, 2013), pp. 346-349.

51. T. Pfeiffer, S. Schuster, S. Bonhoeffer, Cooperation and competition in the evolution of ATP-producing pathways. Science 292, 504-507 (2001).

52. V. Rangaraju, N. Calloway, T. A. Ryan, Activity-driven local ATP synthesis is required for synaptic function. Cell 156, 825-835 (2014).

53. H. R. Knull, Compartmentation of Glycolytic-Enzymes in Nerve-Endings as Determined by Glutaraldehyde Fixation. Journal of Biological Chemistry 255, 6439-6444 (1980).

54. S. Takamori et al., Molecular anatomy of a trafficking organelle. Cell 127, 831-846 (2006).

55. A. Ikemoto, D. G. Bole, T. Ueda, Glycolysis and glutamate accumulation into synaptic vesicles. Role of glyceraldehyde phosphate dehydrogenase and 3-phosphoglycerate kinase. J Biol Chem 278, 5929-5940 (2003).

56. S. Jang et al., Glycolytic Enzymes Localize to Synapses under Energy Stress to Support Synaptic Function. Neuron 90, 278-291 (2016).

57. R. W. Mercer, P. B. Dunham, Membrane-bound ATP fuels the Na/K pump. Studies on membrane-bound glycolytic enzymes on inside-out vesicles from human red cell membranes. J Gen Physiol 78, 547-568 (1981).

58. E. G. Neal et al., The ketogenic diet for the treatment of childhood epilepsy: a randomised controlled trial. The Lancet Neurology 7, 500-506 (2008).

59. W. Ma, J. Berg, G. Yellen, Ketogenic diet metabolites reduce firing in central neurons by opening K(ATP) channels. J Neurosci 27, 3618-3625 (2007).

60. G. Ashrafi, J. de Juan-Sanz, R. J. Farrell, T. A. Ryan, Molecular Tuning of the Axonal Mitochondrial $\mathrm{Ca}(2+)$ Uniporter Ensures Metabolic Flexibility of Neurotransmission. Neuron 105, 678-687 e675 (2020).

61. D. L. Johannsen, E. Ravussin, The role of mitochondria in health and disease. Curr Opin Pharmacol 9, 780-786 (2009).

62. P. M. Herst, M. R. Rowe, G. M. Carson, M. V. Berridge, Functional Mitochondria in Health and Disease. Front Endocrinol (Lausanne) 8, 296 (2017).

63. G. A. Perkins, T. G. Frey, Recent structural insight into mitochondria gained by microscopy. Micron 31, 97-111 (2000).

64. J. R. Friedman, A. Mourier, J. Yamada, J. M. McCaffery, J. Nunnari, MICOS coordinates with respiratory complexes and lipids to establish mitochondrial inner membrane architecture. Elife 4, (2015). 
65. A. S. Moore, E. L. F. Holzbaur, Mitochondrial-cytoskeletal interactions: dynamic associations that facilitate network function and remodeling. Curr Opin Physiol 3, 94100 (2018).

66. I. R. Boldogh, L. A. Pon, Interactions of mitochondria with the actin cytoskeleton. Biochimica et Biophysica Acta (BBA) - Molecular Cell Research 1763, 450-462 (2006).

67. V. Anesti, L. Scorrano, The relationship between mitochondrial shape and function and the cytoskeleton. Biochimica et Biophysica Acta (BBA) - Bioenergetics 1757, 692-699 (2006).

68. L. M. Kraft, L. L. Lackner, Mitochondrial anchors: Positioning mitochondria and more. Biochem Biophys Res Commun 500, 2-8 (2018).

69. Z.-H. Sheng, Mitochondrial trafficking and anchoring in neurons: New insight and implications. The Journal of cell biology 204, 1087-1098 (2014).

70. Q. Cai, Z.-H. Sheng, Moving or stopping mitochondria: Miro as a traffic cop by sensing calcium. Neuron 61, 493-496 (2009).

71. M. C. Avendano-Monsalve, J. C. Ponce-Rojas, S. Funes, From cytosol to mitochondria: the beginning of a protein journey. Biol Chem 401, 645-661 (2020).

72. N. Pfanner, B. Warscheid, N. Wiedemann, Mitochondrial proteins: from biogenesis to functional networks. Nat Rev Mol Cell Biol 20, 267-284 (2019).

73. F. Palmieri, M. Monné, Discoveries, metabolic roles and diseases of mitochondrial carriers: A review. Biochimica et Biophysica Acta (BBA) - Molecular Cell Research 1863, 2362-2378 (2016).

74. M. Monné, A. Vozza, F. M. Lasorsa, V. Porcelli, F. Palmieri, Mitochondrial Carriers for Aspartate, Glutamate and Other Amino Acids: A Review. Int J Mol Sci 20, 4456 (2019).

75. E. B. Taylor, Functional Properties of the Mitochondrial Carrier System. Trends Cell Biol 27, 633-644 (2017).

76. I. Szabo, M. Zoratti, Mitochondrial channels: ion fluxes and more. Physiol Rev 94, 519608 (2014).

77. D. Ponnalagu, H. Singh, in Pharmacology of Mitochondria, H. Singh, S.-S. Sheu, Eds. (Springer International Publishing, Cham, 2017), pp. 71-101.

78. J. M. Baughman et al., Integrative genomics identifies MCU as an essential component of the mitochondrial calcium uniporter. Nature 476, 341-345 (2011).

79. D. De Stefani, A. Raffaello, E. Teardo, I. Szabo, R. Rizzuto, A forty-kilodalton protein of the inner membrane is the mitochondrial calcium uniporter. Nature 476, 336-340 (2011).

80. L. Colina-Tenorio, P. Horten, N. Pfanner, H. Rampelt, Shaping the mitochondrial inner membrane in health and disease. J Intern Med 287, 645-664 (2020). 
81. J. K. Pape et al., Multicolor 3D MINFLUX nanoscopy of mitochondrial MICOS proteins. Proc Natl Acad Sci U S A 117, 20607-20614 (2020).

82. S. Khosravi, M. E. Harner, The MICOS complex, a structural element of mitochondria with versatile functions. Biol Chem 401, 765-778 (2020).

83. T. Stephan et al., MICOS assembly controls mitochondrial inner membrane remodeling and crista junction redistribution to mediate cristae formation. EMBO $J$ 39, e104105 (2020).

84. J. S. Sousa, E. D'Imprima, J. Vonck, in Membrane Protein Complexes: Structure and Function, J. R. Harris, E. J. Boekema, Eds. (Springer Singapore, Singapore, 2018), pp. 167-227.

85. R. Z. Zhao, S. Jiang, L. Zhang, Z. B. Yu, Mitochondrial electron transport chain, ROS generation and uncoupling (Review). Int J Mol Med 44, 3-15 (2019).

86. H. Schagger, K. Pfeiffer, Supercomplexes in the respiratory chains of yeast and mammalian mitochondria. EMBO J 19, 1777-1783 (2000).

87. R. Acin-Perez, P. Fernandez-Silva, M. L. Peleato, A. Perez-Martos, J. A. Enriquez, Respiratory active mitochondrial supercomplexes. Mol Cell 32, 529-539 (2008).

88. P. Tan, Z. Feng, L. Zhang, T. Hou, Y. Li, The mechanism of proton translocation in respiratory complex I from molecular dynamics. J Recept Signal Transduct Res 35, 170-179 (2015).

89. B. Chance, G. R. Williams, Respiratory enzymes in oxidative phosphorylation. IV. The respiratory chain. J Biol Chem 217, 429-438 (1955).

90. B. L. Trumpower, A concerted, alternating sites mechanism of ubiquinol oxidation by the dimeric cytochrome bc1 complex. Biochimica et Biophysica Acta (BBA) Bioenergetics 1555, 166-173 (2002).

91. M. K. Wikstrom, Proton pump coupled to cytochrome c oxidase in mitochondria. Nature 266, 271-273 (1977).

92. A. I. Jonckheere, J. A. Smeitink, R. J. Rodenburg, Mitochondrial ATP synthase: architecture, function and pathology. $J$ Inherit Metab Dis 35, 211-225 (2012).

93. M. S. Patel, N. S. Nemeria, W. Furey, F. Jordan, The pyruvate dehydrogenase complexes: structure-based function and regulation. J Biol Chem 289, 16615-16623 (2014).

94. M. E. Raichle, D. A. Gusnard, Appraising the brain's energy budget. Proc Natl Acad Sci U S A 99, 10237-10239 (2002).

95. M. Picard, B. S. McEwen, Mitochondria impact brain function and cognition. Proceedings of the National Academy of Sciences 111, 7-8 (2014). 
96. T. L. Lewis, Jr., S. K. Kwon, A. Lee, R. Shaw, F. Polleux, MFF-dependent mitochondrial fission regulates presynaptic release and axon branching by limiting axonal mitochondria size. Nat Commun 9, 5008 (2018).

97. A. F. MacAskill, J. T. Kittler, Control of mitochondrial transport and localization in neurons. Trends Cell Biol 20, 102-112 (2010).

98. R. M. Lees, J. D. Johnson, M. C. Ashby, Presynaptic Boutons That Contain Mitochondria Are More Stable. Frontiers in Synaptic Neuroscience 11, (2020).

99. G. M. G. Shepherd, K. M. Harris, Three-Dimensional Structure and Composition of $\mathrm{CA3} \rightarrow \mathrm{CA} 1$ Axons in Rat Hippocampal Slices: Implications for Presynaptic Connectivity and Compartmentalization. The Journal of Neuroscience 18, 8300-8310 (1998).

100. L. Smit-Rigter et al., Mitochondrial Dynamics in Visual Cortex Are Limited In Vivo and Not Affected by Axonal Structural Plasticity. Curr Biol 26, 2609-2616 (2016).

101. K. Obashi, S. Okabe, Regulation of mitochondrial dynamics and distribution by synapse position and neuronal activity in the axon. Eur J Neurosci 38, 2350-2363 (2013).

102. D. Pathak et al., The role of mitochondrially derived ATP in synaptic vesicle recycling. J Biol Chem 290, 22325-22336 (2015).

103. G. A. Perkins et al., The micro-architecture of mitochondria at active zones: electron tomography reveals novel anchoring scaffolds and cristae structured for high-rate metabolism. J Neurosci 30, 1015-1026 (2010).

104. D. T. Chang, A. S. Honick, I. J. Reynolds, Mitochondrial trafficking to synapses in cultured primary cortical neurons. J Neurosci 26, 7035-7045 (2006).

105. M. Sajic et al., Impulse conduction increases mitochondrial transport in adult mammalian peripheral nerves in vivo. PLoS Biol11, e1001754 (2013).

106. T. L. Lewis, Jr., G. F. Turi, S. K. Kwon, A. Losonczy, F. Polleux, Progressive Decrease of Mitochondrial Motility during Maturation of Cortical Axons In Vitro and In Vivo. Curr Biol 26, 2602-2608 (2016).

107. Y. Takihara et al., In vivo imaging of axonal transport of mitochondria in the diseased and aged mammalian CNS. Proc Natl Acad Sci U S A 112, 10515-10520 (2015).

108. H. L. Smith et al., Mitochondrial support of persistent presynaptic vesicle mobilization with age-dependent synaptic growth after LTP. Elife 5, (2016).

109. A. R. Fenton, T. A. Jongens, E. L. F. Holzbaur, Mitochondrial dynamics: Shaping and remodeling an organelle network. Curr Opin Cell Biol 68, 28-36 (2020).

110. J. H. Seo et al., Syntaphilin Ubiquitination Regulates Mitochondrial Dynamics and Tumor Cell Movements. Cancer Res 78, 4215-4228 (2018).

111. C. I. Thomas et al., Presynaptic Mitochondria Volume and Abundance Increase during Development of a High-Fidelity Synapse. J Neurosci 39, 7994-8012 (2019). 
112. C. Cserep, B. Posfai, A. D. Schwarcz, A. Denes, Mitochondrial Ultrastructure Is Coupled to Synaptic Performance at Axonal Release Sites. eNeuro 5, (2018).

113. D. Hopwood, The reactions between formaldehyde, glutaraldehyde and osmium tetroxide, and their fixation effects o bovine serum albumin and on tissue blocks. Histochemie 24, 50-64 (1970).

114. M. L. Watson Staining of Tissue Sections for Electron Microscopy with Heavy Metals. The Journal of Biophysical and Biochemical Cytology 4, 475-478 (1958).

115. K. Yamaguchi, K. Suzuki, K. Tanaka, Examination of electron stains as a substitute for uranyl acetate for the ultrathin sections of bacterial cells. J Electron Microsc (Tokyo) 59, 113-118 (2010).

116. M. Winey, J. B. Meehl, E. T. O'Toole, J. Thomas H. Giddings, Conventional transmission electron microscopy. Molecular Biology of the Cell 25, 319-323 (2014).

117. J. Huebinger, J. Spindler, K. J. Holl, B. Koos, Quantification of protein mobility and associated reshuffling of cytoplasm during chemical fixation. Sci Rep 8, 17756 (2018).

118. E. Kellenberger et al., Artefacts and morphological changes during chemical fixation. $J$ Microsc 168, 181-201 (1992).

119. R. A. Steinbrecht, K. Zierold, Cryotechniques in Biological Electron Microscopy. (1987).

120. C. Bouchet-Marquis, A. Hoenger, Cryo-electron tomography on vitrified sections: a critical analysis of benefits and limitations for structural cell biology. Micron 42, 152162 (2011).

121. A. Al-Amoudi et al., Cryo-electron microscopy of vitreous sections. EMBO J 23, 35833588 (2004).

122. R. N. Irobalieva, B. Martins, O. Medalia, Cellular structural biology as revealed by cryoelectron tomography. J Cell Sci 129, 469-476 (2016).

123. E. Nogales, S. H. Scheres, Cryo-EM: A Unique Tool for the Visualization of Macromolecular Complexity. Mol Cell 58, 677-689 (2015).

124. H. Moor, in Cryotechniques in Biological Electron Microscopy. (1987), chap. Chapter 8, pp. 175-191.

125. D. Studer, B. M. Humbel, M. Chiquet, Electron microscopy of high pressure frozen samples: bridging the gap between cellular ultrastructure and atomic resolution. Histochem Cell Biol 130, 877-889 (2008).

126. J. C. Gilkey, L. A. Staehelin, Advances in ultrarapid freezing for the preservation of cellular ultrastructure. Journal of Electron Microscopy Technique 3, 177-210 (1986).

127. L. Maus et al., Ultrastructural Correlates of Presynaptic Functional Heterogeneity in Hippocampal Synapses. Cell Rep 30, 3632-3643 e3638 (2020). 
128. C. Imig et al., Ultrastructural Imaging of Activity-Dependent Synaptic MembraneTrafficking Events in Cultured Brain Slices. Neuron, (2020).

129. R. Fernandez-Busnadiego et al., Cryo-electron tomography reveals a critical role of RIM1alpha in synaptic vesicle tethering. J Cell Biol 201, 725-740 (2013).

130. R. Fernandez-Busnadiego et al., Insights into the molecular organization of the neuron by cryo-electron tomography. J Electron Microsc (Tokyo) 60 Suppl 1, S137-148 (2011).

131. T. D. Fischer, P. K. Dash, J. Liu, M. N. Waxham, Morphology of mitochondria in spatially restricted axons revealed by cryo-electron tomography. PLoS Biol 16, e2006169 (2018).

132. M. D. Kolba et al., Tunneling nanotube-mediated intercellular vesicle and protein transfer in the stroma-provided imatinib resistance in chronic myeloid leukemia cells. Cell Death Dis 10, 817 (2019).

133. A. Sartori-Rupp et al., Correlative cryo-electron microscopy reveals the structure of TNTs in neuronal cells. Nat Commun 10, 342 (2019).

134. C. S. Xu et al., Enhanced FIB-SEM systems for large-volume 3D imaging. Elife 6, (2017).

135. A. L. Eberle, D. Zeidler, Multi-Beam Scanning Electron Microscopy for HighThroughput Imaging in Connectomics Research. Front Neuroanat 12, 112 (2018).

136. S. J. Park, A. Schertel, K. E. Lee, S. S. Han, Ultra-structural analysis of the brain in a Drosophila model of Alzheimer's disease using FIB/SEM microscopy. Microscopy (Oxf) 63, 3-13 (2014).

137. K. J. Hayworth et al., Gas cluster ion beam SEM for imaging of large tissue samples with $10 \mathrm{~nm}$ isotropic resolution. Nat Methods 17, 68-71 (2020).

138. K. R. Porter, A. Claude, E. F. Fullam, A STUDY OF TISSUE CULTURE CELLS BY ELECTRON MICROSCOPY : METHODS AND PRELIMINARY OBSERVATIONS. $J$ Exp Med 81, 233-246 (1945).

139. O. Shimomura, F. H. Johnson, Y. Saiga, Extraction, purification and properties of aequorin, a bioluminescent protein from the luminous hydromedusan, Aequorea. J Cell Comp Physiol 59, 223-239 (1962).

140. D. P. Hoffman et al., Correlative three-dimensional super-resolution and block-face electron microscopy of whole vitreously frozen cells. Science 367, (2020).

141. S. Watanabe et al., Protein localization in electron micrographs using fluorescence nanoscopy. Nat Methods 8, 80-84 (2011).

142. Y. S. Bykov, M. Cortese, J. A. Briggs, R. Bartenschlager, Correlative light and electron microscopy methods for the study of virus-cell interactions. FEBS Lett 590, 1877-1895 (2016). 
143. S. Saitoh, in Electron Microscopy - Novel Microscopy Trends. (2019), chap. Chapter 3.

144. X. Heiligenstein et al., The HPM Live $\mu$-From Live Cell Imaging to High Pressure Freezing in Less than 2 Seconds for Correlative Microscopy Approaches. Microscopy and Microanalysis 23, 1276-1277 (2017).

145. F. Lange et al., (2020).

146. W. Kukulski, M. Schorb, M. Kaksonen, J. A. Briggs, Plasma membrane reshaping during endocytosis is revealed by time-resolved electron tomography. Cell 150, 508520 (2012).

147. A. Picco et al., The contributions of the actin machinery to endocytic membrane bending and vesicle formation. Mol Biol Cell 29, 1346-1358 (2018).

148. Y. S. Bykov et al., High-throughput ultrastructure screening using electron microscopy and fluorescent barcoding. J Cell Biol 218, 2797-2811 (2019).

149. R. Sun et al., Correlative light and electron microscopy for complex cellular structures on PDMS substrates with coded micro-patterns. Lab Chip 18, 3840-3848 (2018).

150. R. Sun et al., An efficient protocol of cryo-correlative light and electron microscopy for the study of neuronal synapses. Biophysics Reports 5, 111-122 (2019).

151. J. E. Reilly et al., Characterization of MSB synapses in dissociated hippocampal culture with simultaneous pre- and postsynaptic live microscopy. PLoS One 6, e26478 (2011).

152. P. Paul-Gilloteaux et al., eC-CLEM: flexible multidimensional registration software for correlative microscopies. Nat Methods 14, 102-103 (2017).

153. C. A. Schneider, W. S. Rasband, K. W. Eliceiri, NIH Image to ImageJ: 25 years of image analysis. Nat Methods 9, 671-675 (2012).

154. Y. Hua, P. Laserstein, M. Helmstaedter, Large-volume en-bloc staining for electron microscopy-based connectomics. Nat Commun 6, 7923 (2015).

155. C. Genoud, B. Titze, A. Graff-Meyer, R. W. Friedrich, Fast Homogeneous En Bloc Staining of Large Tissue Samples for Volume Electron Microscopy. Front Neuroanat 12, 76 (2018).

156. A. A. Cluntun, M. J. Lukey, R. A. Cerione, J. W. Locasale, Glutamine Metabolism in Cancer: Understanding the Heterogeneity. Trends in Cancer 3, 169-180 (2017).

157. W. Kuhlbrandt, Structure and function of mitochondrial membrane protein complexes. BMC Biol 13, 89 (2015).

158. J. R. Kremer, D. N. Mastronarde, J. R. McIntosh, Computer visualization of threedimensional image data using IMOD. J Struct Biol 116, 71-76 (1996).

159. C. Wang, R. J. Youle, The role of mitochondria in apoptosis*. Annu Rev Genet 43, 95118 (2009). 
160. S. S. Smaili, Y. T. Hsu, K. M. Sanders, J. T. Russell, R. J. Youle, Bax translocation to mitochondria subsequent to a rapid loss of mitochondrial membrane potential. Cell Death Differ 8, 909-920 (2001).

161. D. Garenne, T. T. Renault, S. Manon, Bax mitochondrial relocation is linked to its phosphorylation and its interaction with Bcl-xL. Microb Cell 3, 597-605 (2016).

162. L. Grosse et al., Bax assembles into large ring-like structures remodeling the mitochondrial outer membrane in apoptosis. EMBO J 35, 402-413 (2016).

163. A. Sesso et al., Mitochondrial Swelling and Incipient Outer Membrane Rupture in Preapoptotic and Apoptotic Cells. Anat Rec 295, 1647-1659 (2012).

164. I. Belevich, M. Joensuu, D. Kumar, H. Vihinen, E. Jokitalo, Microscopy Image Browser: A Platform for Segmentation and Analysis of Multidimensional Datasets. PLoS Biol 14, e1002340 (2016).

165. M. E. Maes, C. L. Schlamp, R. W. Nickells, Live-cell imaging to measure BAX recruitment kinetics to mitochondria during apoptosis. PLoS One 12, e0184434 (2017).

166. M. Karbowski, R. J. Youle, Dynamics of mitochondrial morphology in healthy cells and during apoptosis. Cell Death Differ 10, 870-880 (2003).

167. R. Salvador-Gallego et al., Bax assembly into rings and arcs in apoptotic mitochondria is linked to membrane pores. EMBO J 35, 389-401 (2016).

168. E. Johnson et al., Correlative in-resin super-resolution and electron microscopy using standard fluorescent proteins. Sci Rep 5, 9583 (2015).

169. W. Kukulski et al., Precise, correlated fluorescence microscopy and electron tomography of lowicryl sections using fluorescent fiducial markers. Methods Cell Biol 111, 235-257 (2012).

170. R. Dahl, L. A. Staehelin, High-pressure freezing for the preservation of biological structure: theory and practice. J Electron Microsc Tech 13, 165-174 (1989).

171. P. Rostaing, R. M. Weimer, E. M. Jorgensen, A. Triller, J. L. Bessereau, Preservation of immunoreactivity and fine structure of adult $\mathrm{C}$. elegans tissues using high-pressure freezing. J Histochem Cytochem 52, 1-12 (2004).

172. M. W. Tuijtel et al., Inducing fluorescence of uranyl acetate as a dual-purpose contrast agent for correlative light-electron microscopy with nanometre precision. Sci Rep 7, 10442 (2017).

173. C. Xintaropoulou et al., A comparative analysis of inhibitors of the glycolysis pathway in breast and ovarian cancer cell line models. Oncotarget 6, 25677-25695 (2015).

174. M. I. Sabri, S. Ochs, Inhibition of glyceraldehyde-3-phosphate dehydrogenase in mammalian nerve by iodoacetic acid. J Neurochem 18, 1509-1514 (1971). 
175. J. R. Williamson, Glycolytic control mechanisms. 3. Effects of iodoacetamide and fluoroacetate on glucose metabolism in the perfused rat heart. J Biol Chem 242, 4476 4485 (1967).

176. B. Pajak et al., 2-Deoxy-d-Glucose and Its Analogs: From Diagnostic to Therapeutic Agents. Int J Mol Sci 21, (2019).

177. M. M. Schmidt, R. Dringen, Differential effects of iodoacetamide and iodoacetate on glycolysis and glutathione metabolism of cultured astrocytes. Front Neuroenergetics $\mathbf{1}$, 1 (2009).

178. W. van der Stel et al., Multiparametric assessment of mitochondrial respiratory inhibition in HepG2 and RPTEC/TERT1 cells using a panel of mitochondrial targeting agrochemicals. Arch Toxicol 94, 2707-2729 (2020).

179. C. W. Huang, K. M. Lin, T. Y. Hung, Y. C. Chuang, S. N. Wu, Multiple Actions of Rotenone, an Inhibitor of Mitochondrial Respiratory Chain, on lonic Currents and Miniature End-Plate Potential in Mouse Hippocampal (mHippoE-14) Neurons. Cell Physiol Biochem 47, 330-343 (2018).

180. C. Ballot et al., Inhibition of mitochondrial respiration mediates apoptosis induced by the anti-tumoral alkaloid lamellarin D. Apoptosis 15, 769-781 (2010).

181. H. Pelicano et al., Inhibition of mitochondrial respiration: a novel strategy to enhance drug-induced apoptosis in human leukemia cells by a reactive oxygen speciesmediated mechanism. J Biol Chem 278, 37832-37839 (2003).

182. A. R. Diers, K. A. Broniowska, C. F. Chang, N. Hogg, Pyruvate fuels mitochondrial respiration and proliferation of breast cancer cells: effect of monocarboxylate transporter inhibition. Biochem J 444, 561-571 (2012).

183. K. Tieu et al., D-beta-hydroxybutyrate rescues mitochondrial respiration and mitigates features of Parkinson disease. J Clin Invest 112, 892-901 (2003).

184. S. C. Choi, J. C. Hall, A study of respiration and oxidative phosphorylation of mitochondria from regenerating liver of normal and diabetic rats. Cancer Res 34, 23512357 (1974).

185. L. B. Achanta, B. D. Rowlands, D. S. Thomas, G. D. Housley, C. D. Rae, betaHydroxybutyrate Boosts Mitochondrial and Neuronal Metabolism but is not Preferred Over Glucose Under Activated Conditions. Neurochem Res 42, 1710-1723 (2017).

186. S. Frey et al., The addition of ketone bodies alleviates mitochondrial dysfunction by restoring complex I assembly in a MELAS cellular model. Biochim Biophys Acta Mol Basis Dis 1863, 284-291 (2017).

187. F. Braun, J. Bertin-Ciftci, A.-S. Gallouet, J. Millour, P. Juin, Serum-nutrient starvation induces cell death mediated by Bax and Puma that is counteracted by p21 and unmasked by Bcl-x(L) inhibition. PloS one 6, e23577-e23577 (2011). 
188. A. A. Goyeneche, J. M. Harmon, C. M. Telleria, Cell death induced by serum deprivation in luteal cells involves the intrinsic pathway of apoptosis. Reproduction 131, 103-111 (2006).

189. G. Wang et al., Serum starvation induces cell death in NSCLC via miR-224. Onco Targets Ther 12, 3953-3962 (2019).

190. M. Zhang et al., beta-Hydroxybutyrate Facilitates Fatty Acids Synthesis Mediated by Sterol Regulatory Element-Binding Protein1 in Bovine Mammary Epithelial Cells. Cell Physiol Biochem 37, 2115-2124 (2015).

191. P. M. Sheehan, Y. Y. Yeh, Pathways of acetyl CoA production for lipogenesis from acetoacetate, beta-hydroxybutyrate, pyruvate and glucose in neonatal rat lung. Lipids 19, 103-108 (1984).

192. H. L. Petrick et al., In vitro ketone-supported mitochondrial respiration is minimal when other substrates are readily available in cardiac and skeletal muscle. J Physiol, (2020).

193. J. M. Rho, L. R. Shao, C. E. Stafstrom, 2-Deoxyglucose and Beta-Hydroxybutyrate: Metabolic Agents for Seizure Control. Front Cell Neurosci 13, 172 (2019).

194. F. K. Odorcyk et al., Differential glucose and beta-hydroxybutyrate metabolism confers an intrinsic neuroprotection to the immature brain in a rat model of neonatal hypoxia ischemia. Exp Neurol330, 113317 (2020).

195. Y. M. Han, T. Ramprasath, M. H. Zou, beta-hydroxybutyrate and its metabolic effects on age-associated pathology. Exp Mol Med 52, 548-555 (2020).

196. B. Cuenoud et al., Metabolism of Exogenous D-Beta-Hydroxybutyrate, an Energy Substrate Avidly Consumed by the Heart and Kidney. Front Nutr 7, 13 (2020).

197. J. C. Newman, E. Verdin, $\beta$-Hydroxybutyrate: A Signaling Metabolite. Annu Rev Nutr 37, 51-76 (2017).

198. A. Almansa-Ordonez, R. Bellido, R. Vassena, M. Barragan, F. Zambelli, Oxidative Stress in Reproduction: A Mitochondrial Perspective. Biology (Basel) 9, (2020).

199. D. W. Freeman, R. S. Petralia, Y. X. Wang, M. P. Mattson, P. J. Yao, Mitochondria in hippocampal presynaptic and postsynaptic compartments differ in size as well as intensity. Matters (Zur) 2017, (2017).

200. T. K. Tsang et al., High-quality ultrastructural preservation using cryofixation for 3D electron microscopy of genetically labeled tissues. Elife 7, (2018).

201. C. A. Mannella, The relevance of mitochondrial membrane topology to mitochondrial function. Biochimica et Biophysica Acta (BBA) - Molecular Basis of Disease 1762, 140 147 (2006).

202. T. Delgado et al., Comparing 3D ultrastructure of presynaptic and postsynaptic mitochondria. Biol Open 8, (2019). 
203. R. Seager, L. Lee, J. M. Henley, K. A. Wilkinson, Mechanisms and roles of mitochondrial localisation and dynamics in neuronal function. Neuronal Signal 4, NS20200008 (2020).

204. S. Milde, R. Adalbert, M. H. Elaman, M. P. Coleman, Axonal transport declines with age in two distinct phases separated by a period of relative stability. Neurobiol Aging 36, 971-981 (2015).

205. L. Brodin, L. Bakeeva, O. Shupliakov, Presynaptic mitochondria and the temporal pattern of neurotransmitter release. Philos Trans $R$ Soc Lond B Biol Sci 354, 365-372 (1999).

206. T. L. Schwarz, Mitochondrial trafficking in neurons. Cold Spring Harb Perspect Bio/ 5, (2013).

207. M. V. Green et al., Automated Live-Cell Imaging of Synapses in Rat and Human Neuronal Cultures. Frontiers in Cellular Neuroscience 13, (2019).

208. G. Ashrafi, T. A. Ryan, Glucose metabolism in nerve terminals. Curr Opin Neurobiol 45, 156-161 (2017).

209. E. Molnar, Long-term potentiation in cultured hippocampal neurons. Semin Cell Dev Biol 22, 506-513 (2011).

210. W. Macías, R. Carlson, A. Rajadhyaksha, A. Barczak, C. Konradi, Potassium chloride depolarization mediates CREB phosphorylation in striatal neurons in an NMDA receptor-dependent manner. Brain Research 890, 222-232 (2001).

211. E. J. Kilbourne, A. McMahon, E. L. Sabban, Membrane depolarization by isotonic or hypertonic $\mathrm{KCl}$ : differential effects on mRNA levels of tyrosine hydroxylase and dopamine $\beta$-hydroxylase mRNA in PC12 cells. Journal of Neuroscience Methods 40, 193-202 (1991).

212. J. I. Aguilar et al., Neuronal Depolarization Drives Increased Dopamine Synaptic Vesicle Loading via VGLUT. Neuron 95, 1074-1088 e1077 (2017).

213. X. B. He et al., Prolonged membrane depolarization enhances midbrain dopamine neuron differentiation via epigenetic histone modifications. Stem Cells 29, 1861-1873 (2011).

214. G.-q. Bi, M.-m. Poo, Synaptic Modifications in Cultured Hippocampal Neurons: Dependence on Spike Timing, Synaptic Strength, and Postsynaptic Cell Type. The Journal of Neuroscience 18, 10464-10472 (1998).

215. J. Hogins, D. C. Crawford, C. F. Zorumski, S. Mennerick, Excitotoxicity Triggered by Neurobasal Culture Medium. PLOS ONE 6, e25633 (2011).

216. Z. H. Sheng, Mitochondrial trafficking and anchoring in neurons: New insight and implications. J Cell Biol 204, 1087-1098 (2014). 
217. S. Takamori, J. S. Rhee, C. Rosenmund, R. Jahn, Identification of a vesicular glutamate transporter that defines a glutamatergic phenotype in neurons. Nature 407, 189-194 (2000).

218. E. E. Bellocchio, R. J. Reimer, R. T. Fremeau, Jr., R. H. Edwards, Uptake of glutamate into synaptic vesicles by an inorganic phosphate transporter. Science 289, 957-960 (2000).

219. F. Bai, F. A. Witzmann, Synaptosome proteomics. Subcell Biochem 43, 77-98 (2007).

220. J. DiGiovanni, T. Sun, Z.-H. Sheng, Characterizing Synaptic Vesicle Proteins Using Synaptosomal Fractions and Cultured Hippocampal Neurons. Current Protocols in Neuroscience 59, 2.7.1-2.7.22 (2012).

221. M. Agnello, G. Morici, A. M. Rinaldi, A method for measuring mitochondrial mass and activity. Cytotechnology 56, 145-149 (2008).

222. A. P. Leonard et al., Quantitative analysis of mitochondrial morphology and membrane potential in living cells using high-content imaging, machine learning, and morphological binning. Biochim Biophys Acta 1853, 348-360 (2015).

223. S. M. Ribeiro, A. Gimenez-Cassina, N. N. Danial, Measurement of mitochondrial oxygen consumption rates in mouse primary neurons and astrocytes. Methods Mol Biol 1241, 59-69 (2015).

224. M. Gleichmann, L. P. Collis, P. J. Smith, M. P. Mattson, Simultaneous single neuron recording of $\mathrm{O} 2$ consumption, [Ca2+]i and mitochondrial membrane potential in glutamate toxicity. J Neurochem 109, 644-655 (2009).

225. G. Knott, S. Rosset, M. Cantoni, Focussed ion beam milling and scanning electron microscopy of brain tissue. J Vis Exp, e2588 (2011).

226. Y. Kubota, J. Sohn, Y. Kawaguchi, Large Volume Electron Microscopy and Neural Microcircuit Analysis. Front Neural Circuits 12, 98 (2018).

227. L. Song, J. McGee, E. J. Walsh, Frequency- and level-dependent changes in auditory brainstem responses (ABRS) in developing mice. J Acoust Soc Am 119, 2242-2257 (2006).

228. M. Mao, J. M. Montgomery, M. F. Kubke, P. R. Thorne, The Structural Development of the Mouse Dorsal Cochlear Nucleus. J Assoc Res Otolaryngol 16, 473-486 (2015).

229. V. Chavan et al., Central Presynaptic Terminals Are Enriched in ATP but the Majority Lack Mitochondria. PLOS ONE 10, e0125185 (2015).

230. A. Dosemeci, R. J. Weinberg, T. S. Reese, J. H. Tao-Cheng, The Postsynaptic Density: There Is More than Meets the Eye. Front Synaptic Neurosci 8, 23 (2016).

231. J. H. Tao-Cheng, Stimulation induces gradual increases in the thickness and curvature of postsynaptic density of hippocampal CA1 neurons in slice cultures. Mol Brain 12, 44 (2019). 
232. R. M. Lees, J. D. Johnson, M. C. Ashby, Presynaptic Boutons That Contain Mitochondria Are More Stable. Front Synaptic Neurosci 11, 37 (2019).

233. J. Shen, M. T. Colonnese, Development of Activity in the Mouse Visual Cortex. $J$ Neurosci 36, 12259-12275 (2016).

234. B. S. Padman, M. Bach, G. Ramm, An improved procedure for subcellular spatial alignment during live-cell CLEM. PLoS One 9, e95967 (2014).

235. X. Heiligenstein et al., The CryoCapsule: simplifying correlative light to electron microscopy. Traffic 15, 700-716 (2014).

236. A. Sesso et al., Mitochondrial swelling and incipient outer membrane rupture in preapoptotic and apoptotic cells. Anat Rec (Hoboken) 295, 1647-1659 (2012).

237. K. McArthur et al., BAK/BAX macropores facilitate mitochondrial herniation and mtDNA efflux during apoptosis. Science 359, (2018).

238. N. R. Ader et al., Molecular and topological reorganizations in mitochondrial architecture interplay during Bax-mediated steps of apoptosis. Elife 8, (2019).

239. S. Mohammadian et al., High accuracy, fiducial marker-based image registration of correlative microscopy images. Sci Rep 9, 3211 (2019).

240. C. A. Wurm et al., Correlative STED super-resolution light and electron microscopy on resin sections. Journal of Physics D: Applied Physics 52, (2019).

241. W. Mobius, G. Posthuma, Sugar and ice: Immunoelectron microscopy using cryosections according to the Tokuyasu method. Tissue Cell 57, 90-102 (2019).

242. G. Griffiths, J. M. Lucocq, Antibodies for immunolabeling by light and electron microscopy: not for the faint hearted. Histochem Cell Biol 142, 347-360 (2014).

243. M. Bowler et al., High-resolution characterization of centriole distal appendage morphology and dynamics by correlative STORM and electron microscopy. Nat Commun 10, 993 (2019).

244. S. E. Senyo et al., Mammalian heart renewal by pre-existing cardiomyocytes. Nature 493, 433-436 (2013).

245. I. C. Vreja et al., Secondary-ion mass spectrometry of genetically encoded targets. Angew Chem Int Ed Engl 54, 5784-5788 (2015).

246. S. K. Saka et al., Correlated optical and isotopic nanoscopy. Nat Commun 5, 3664 (2014).

247. E. D. R. Arrojo et al., Age Mosaicism across Multiple Scales in Adult Tissues. Cell Metab 30, 343-351 e343 (2019).

248. B. H. Toyama et al., Visualization of long-lived proteins reveals age mosaicism within nuclei of postmitotic cells. J Cell Biol 218, 433-444 (2019).

249. J. Lovric et al., Nano Secondary Ion Mass Spectrometry Imaging of Dopamine Distribution Across Nanometer Vesicles. ACS Nano 11, 3446-3455 (2017). 

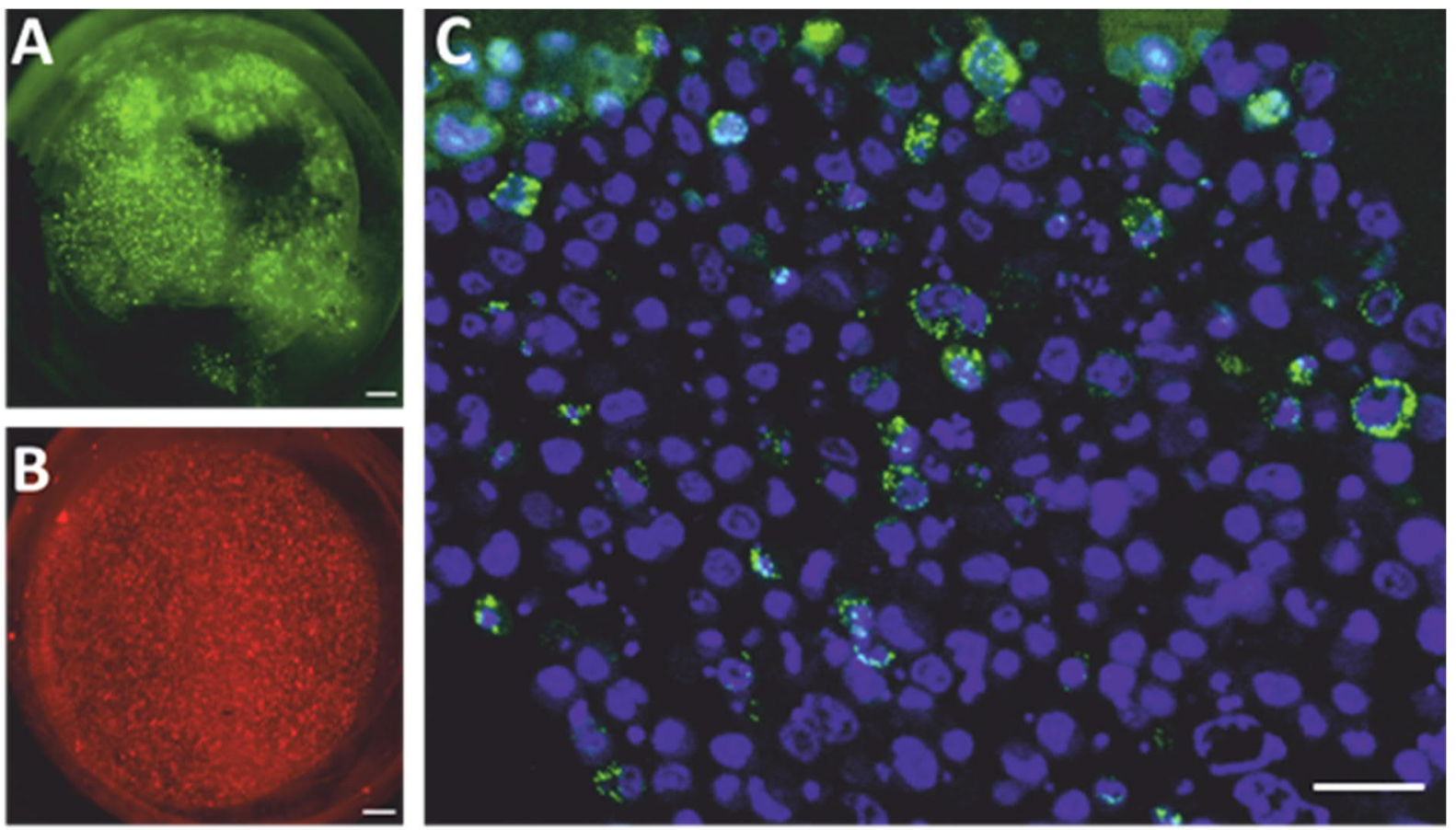

\section{Supplementary images}

Figure 41: Samples for correlative light and electron microscopy. Pellets of HeLa cells prepared for high-accuracy CLEM. A: Overview of a resin block showing fluorescence preservation of mito-mCitrine expressed for 24 hours prior to high-pressure freezing and freeze substitution. Imaged with an epifluorescence microscope equipped with a 10x air objective. B: Overview of a resin block showing fluorescence preservation of mito-mScarlet expressed for 24 hours prior to high-pressure freezing and freeze substitution. Imaged with an epifluorescence microscope equipped with a 10x air objective. C: $400 \mathrm{~nm}$ thick resin section mounted on a glass coverslip and additionally stained with DAPI in PBS prior to imaging in an epifluorescence microscope equipped with a 20x air objective. Green: mito-mCitrine, blue: DAPI. Scale bars in $A$ and $B=$ $500 \mu \mathrm{m}$. Scale bar in $C=50 \mu \mathrm{m}$. 


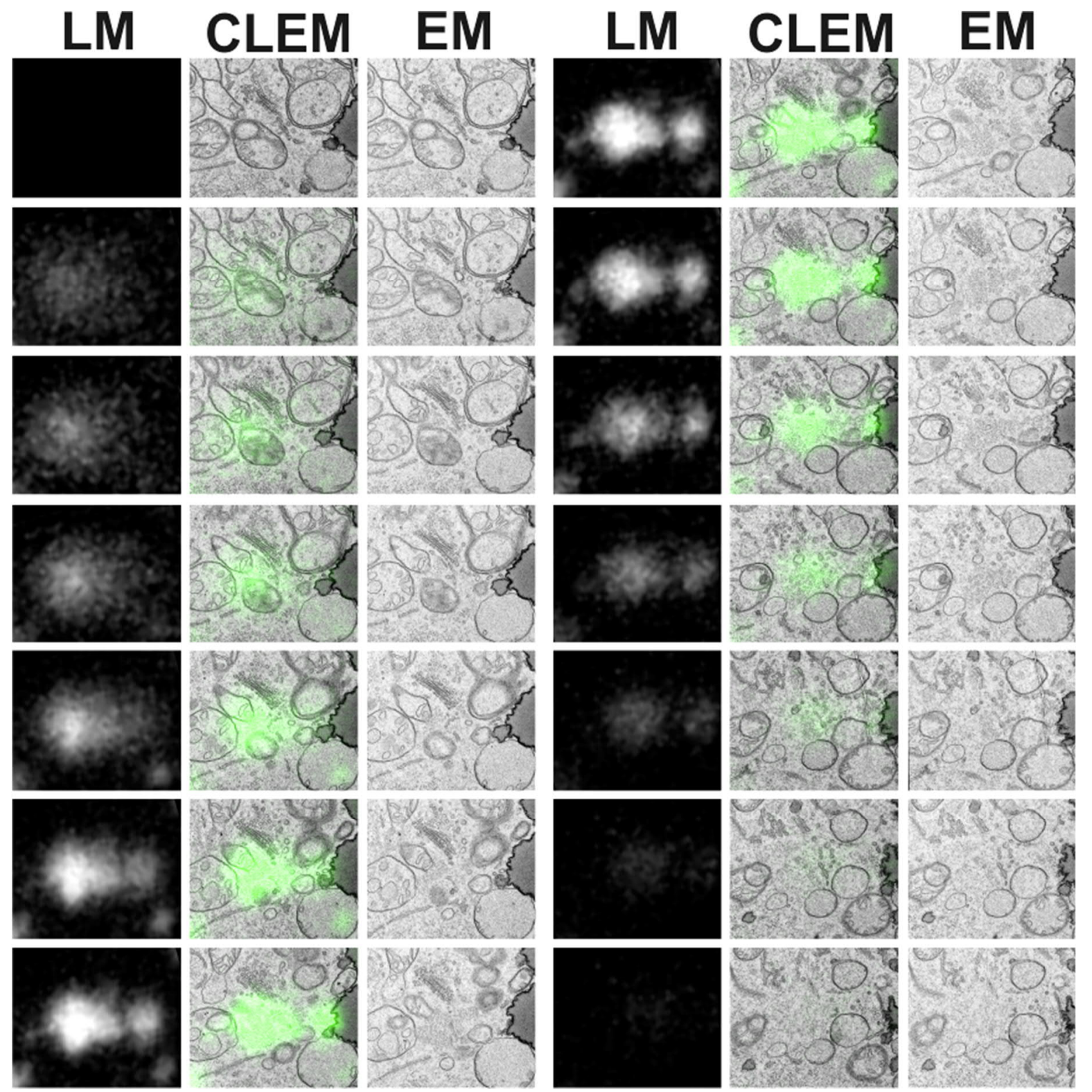

Figure 42: 3D CLEM of Bax-EGFP. Registration of light microscopy images to the electron micrographs resulted in a $3 D$ localization of the EGFP signal in the ultrastructure. $L M$ : the row shows the registered light microscopy signal of EGFP. The images were oversampled to match the resolution of the micrographs during correlation in ICY. CLEM: the row shows the correlative images obtained by image registration. EM: the row shows the micrographs obtained from 14 consecutive resin sections that cover the volume of interest. 


\section{Mitochondrial diameter}

\begin{tabular}{|c|c|c|c|c|c|c|}
\hline \multicolumn{7}{|l|}{ HeLa } \\
\hline & Glucose & Pyruvate & Glucose & Keto & Pyruvate & Keto \\
\hline Mean & 201.09 & 285.55 & 201.09 & 298.30 & 285.55 & 298.30 \\
\hline Variance & 2984.05 & 6860.04 & 2984.05 & 4499.03 & 6860.04 & 4499.03 \\
\hline Observations & 243 & 254 & 243 & 162 & 254 & 162 \\
\hline Hypothesized Mean Difference & 0 & & 0 & & 0 & \\
\hline df & 495 & & 403 & & 414 & \\
\hline t Stat & -13.35835 & & -15.99804 & & -1.645205 & \\
\hline $\mathrm{P}(\mathrm{T}<=\mathrm{t})$ one-tail & $2.814 \mathrm{E}-35$ & & $2.975 \mathrm{E}-45$ & & 0.0503432 & \\
\hline t Critical one-tail & 1.6479378 & & 1.6486435 & & 1.6485425 & \\
\hline $\mathrm{P}(\mathrm{T}<=\mathrm{t})$ two-tail & $5.628 \mathrm{E}-35$ & & $5.951 \mathrm{E}-45$ & & 0.1006865 & \\
\hline t Critical two-tail & 1.964768 & & 1.9658679 & & 1.9657106 & \\
\hline \multicolumn{7}{|l|}{ Cos7 } \\
\hline & Glucose & Pyruvate & Glucose & Keto & Pyruvate & Keto \\
\hline Mean & 220.20 & 339.31 & 220.20 & 361.14 & 339.31 & 361.14 \\
\hline Variance & 3587.48 & 9866.54 & 3587.48 & 9440.16 & 9866.54 & 9440.16 \\
\hline Observations & 186 & 171 & 186 & 142 & 171 & 142 \\
\hline Hypothesized Mean Difference & 0 & & 0 & & 0 & \\
\hline df & 355 & & 326 & & 311 & \\
\hline t Stat & -13.84478 & & -16.16847 & & -1.955041 & \\
\hline $\mathrm{P}(\mathrm{T}<=\mathrm{t})$ one-tail & $1.858 \mathrm{E}-35$ & & $6.824 \mathrm{E}-44$ & & 0.0257368 & \\
\hline t Critical one-tail & 1.6491572 & & 1.6495412 & & 1.6497679 & \\
\hline $\mathrm{P}(\mathrm{T}<=\mathrm{t})$ two-tail & 3.716E-35 & & $1.365 \mathrm{E}-43$ & & 0.0514737 & \\
\hline t Critical two-tail & 1.9666689 & & 1.9672675 & & 1.9676211 & \\
\hline \multicolumn{7}{|l|}{ U-2 OS } \\
\hline & Glucose & Pyruvate & Glucose & Keto & Pyruvate & Keto \\
\hline Mean & 204.59 & 248.68 & 204.59 & 277.68 & 248.68 & 277.68 \\
\hline Variance & 2688.37 & 5177.58 & 2688.37 & 3904.37 & 5177.58 & 3904.37 \\
\hline Observations & 156 & 224 & 156 & 161 & 224 & 161 \\
\hline Hypothesized Mean Difference & 0 & & 0 & & 0 & \\
\hline df & 378 & & 315 & & 383 & \\
\hline t Stat & -6.556701 & & -11.315 & & -4.118975 & \\
\hline $\mathrm{P}(\mathrm{T}<=\mathrm{t})$ one-tail & 9.043E-11 & & 1.943E-25 & & 2.333E-05 & \\
\hline t Critical one-tail & 1.6488947 & & 1.6497053 & & 1.6488418 & \\
\hline $\mathrm{P}(\mathrm{T}<=\mathrm{t})$ two-tail & $1.809 \mathrm{E}-10$ & & $3.886 \mathrm{E}-25$ & & 4.666E-05 & \\
\hline t Critical two-tail & 1.9662596 & & 1.9675235 & & 1.9661772 & \\
\hline
\end{tabular}

Figure 43: Statistical tests for changes of the mitochondrial diameter in HeLa, Cos7 and U-2 OS cells cultivated in SILAC FLEX medium with the indicated carbon sources. All conditions were tested with the "t-test two sample assuming unequal variances" data analysis tool in Excel 2016. 
Crista length per mitochondrial area

\begin{tabular}{|c|c|c|c|c|c|c|}
\hline \multirow{2}{*}{\multicolumn{7}{|c|}{ HeLa }} \\
\hline & & & & & & \\
\hline & Glucose & Pyruvate & Glucose & Keto & Pyruvate & Keto \\
\hline Mean & 4.03 & 5.80 & 4.03 & 6.61 & 5.96 & 6.61 \\
\hline Variance & 2.50 & 2.65 & 2.50 & 3.78 & 7.01 & 3.78 \\
\hline Observations & 236 & 245 & 236 & 162 & 245 & 162 \\
\hline Hypothesized Mean Difference & 0 & & 0 & & 0 & \\
\hline df & 479 & & 396 & & 405 & \\
\hline t Stat & -12.03707 & & -14.5383 & & -2.688519 & \\
\hline $\mathrm{P}(\mathrm{T}<=\mathrm{t})$ one-tail & $1.22 E-29$ & & 5.623E-39 & & 0.0037366 & \\
\hline t Critical one-tail & 1.648041 & & 1.6487106 & & 1.6486247 & \\
\hline $\mathrm{P}(\mathrm{T}<=\mathrm{t})$ two-tail & $2.44 \mathrm{E}-29$ & & 1.125E-38 & & 0.0074732 & \\
\hline t Critical two-tail & 1.9649289 & & 1.9659726 & & 1.9658387 & \\
\hline \multicolumn{7}{|l|}{ Cos7 } \\
\hline & Glucose & Pyruvate & Glucose & Keto & Pyruvate & Keto \\
\hline Mean & 5.80 & 7.73 & 5.80 & 7.81 & 7.73 & 7.81 \\
\hline Variance & 4.10 & 5.07 & 4.10 & 4.31 & 5.07 & 4.31 \\
\hline Observations & 129 & 150 & 129 & 187 & 150 & 187 \\
\hline Hypothesized Mean Difference & 0 & & 0 & & 0 & \\
\hline df & 277 & & 314 & & 335 & \\
\hline t Stat & -7.451066 & & -8.518011 & & -0.340252 & \\
\hline $\mathrm{P}(\mathrm{T}<=\mathrm{t})$ one-tail & $5.922 \mathrm{E}-13$ & & $3.429 \mathrm{E}-16$ & & 0.36694 & \\
\hline t Critical one-tail & 1.6503732 & & 1.6497208 & & 1.6494149 & \\
\hline $\mathrm{P}(\mathrm{T}<=\mathrm{t})$ two-tail & 1.184E-12 & & 6.857E-16 & & 0.7338801 & \\
\hline t Critical two-tail & 1.968565 & & 1.9675477 & & 1.9670706 & \\
\hline \multicolumn{7}{|l|}{ U-2OS } \\
\hline & Glucose & Pyruvate & Glucose & Keto & Pyruvate & Keto \\
\hline Mean & 5.07 & 6.80 & 5.07 & 6.08 & 6.80 & 6.08 \\
\hline Variance & 4.01 & 4.10 & 4.01 & 3.04 & 4.10 & 3.04 \\
\hline Observations & 217 & 103 & 217 & 137 & 103 & 137 \\
\hline Hypothesized Mean Difference & 0 & & 0 & & 0 & \\
\hline$d f$ & 318 & & 352 & & 238 & \\
\hline t Stat & -7.203488 & & -4.861799 & & 2.9590526 & \\
\hline $\mathrm{P}(\mathrm{T}<=\mathrm{t})$ one-tail & $2.139 \mathrm{E}-12$ & & 8.78E-07 & & 0.0016986 & \\
\hline t Critical one-tail & 1.6496594 & & 1.649194 & & 1.6512812 & \\
\hline $\mathrm{P}(\mathrm{T}<=\mathrm{t})$ two-tail & $4.278 \mathrm{E}-12$ & & 1.756E-06 & & 0.0033973 & \\
\hline t Critical two-tail & 1.9674519 & & 1.9667262 & & 1.9699815 & \\
\hline
\end{tabular}

Figure 44: Statistical test for changes in the average crista membrane abundance per mitochondrial area of HeLa, Cos7 and U-2 OS cells grown in SILAC FLEX medium with the indicated carbon sources. All conditions were tested with the "t-test two sample assuming unequal variances" data analysis tool in Excel 2016. 
Crista length per mitochondrial length

\begin{tabular}{|c|c|c|c|c|c|c|}
\hline \multicolumn{7}{|l|}{ HeLa } \\
\hline & Glucose & Pyruvate & Glucose & Keto & Pyruvate & Keto \\
\hline Mean & 0.81 & 1.66 & 0.81 & 1.98 & 1.66 & 1.98 \\
\hline Variance & 0.18 & 0.41 & 0.18 & 0.60 & 0.41 & 0.60 \\
\hline Observations & 236 & 243 & 236 & 162 & 243 & 162 \\
\hline Hypothesized Mean Difference & 0 & & 0 & & 0 & \\
\hline df & 477 & & 396 & & 403 & \\
\hline t Stat & -16.95382 & & -19.25909 & & -4.468757 & \\
\hline $\mathrm{P}(\mathrm{T}<=\mathrm{t})$ one-tail & 4.194E-51 & & $4.191 \mathrm{E}-59$ & & 5.118E-06 & \\
\hline t Critical one-tail & 1.6480544 & & 1.6487106 & & 1.6486435 & \\
\hline $\mathrm{P}(\mathrm{T}<=\mathrm{t})$ two-tail & $8.388 \mathrm{E}-51$ & & 8.383E-59 & & 1.024E-05 & \\
\hline t Critical two-tail & 1.9649497 & & 1.9659726 & & 1.9658679 & \\
\hline \multicolumn{7}{|l|}{ Cos7 } \\
\hline & Glucose & Pyruvate & Glucose & Keto & Pyruvate & Keto \\
\hline Mean & 1.32 & 2.63 & 1.32 & 2.57 & 2.63 & 2.57 \\
\hline Variance & 0.38 & 1.23 & 0.38 & 0.74 & 1.23 & 0.74 \\
\hline Observations & 129 & 150 & 129 & 142 & 150 & 142 \\
\hline Hypothesized Mean Difference & 0 & & 0 & & 0 & \\
\hline df & 277 & & 269 & & 290 & \\
\hline t Stat & -11.92301 & & -13.57888 & & 0.5291432 & \\
\hline $\mathrm{P}(\mathrm{T}<=\mathrm{t})$ one-tail & 4.957E-27 & & 1.217E-32 & & 0.2985555 & \\
\hline t Critical one-tail & 1.6503732 & & 1.6505379 & & 1.6501249 & \\
\hline $\mathrm{P}(\mathrm{T}<=\mathrm{t})$ two-tail & $9.914 \mathrm{E}-27$ & & $2.435 \mathrm{E}-32$ & & 0.5971109 & \\
\hline t Critical two-tail & 1.968565 & & 1.968822 & & 1.9681779 & \\
\hline \multicolumn{7}{|l|}{ U-2 OS } \\
\hline & Glucose & Pyruvate & Glucose & Keto & Pyruvate & Keto \\
\hline Mean & 0.96 & 1.83 & 0.96 & 1.70 & 1.83 & 1.70 \\
\hline Variance & 0.22 & 0.74 & 0.22 & 0.34 & 0.74 & 0.34 \\
\hline Observations & 156 & 103 & 156 & 137 & 103 & 137 \\
\hline Hypothesized Mean Difference & 0 & & 0 & & 0 & \\
\hline df & 257 & & 291 & & 238 & \\
\hline t Stat & -10.57593 & & -12.10005 & & 1.4341207 & \\
\hline $\mathrm{P}(\mathrm{T}<=\mathrm{t})$ one-tail & 3.069E-22 & & 7.084E-28 & & 0.0764252 & \\
\hline t Critical one-tail & 1.6508043 & & 1.6501068 & & 1.6512812 & \\
\hline $\mathrm{P}(\mathrm{T}<=\mathrm{t})$ two-tail & 6.138E-22 & & 1.417E-27 & & 0.1528504 & \\
\hline t Critical two-tail & 1.9692375 & & 1.9681496 & & 1.9699815 & \\
\hline
\end{tabular}

Figure 45: Statistical test for changes in the average crista membrane abundance per mitochondrial length of HeLa, Cos7 and U-2 OS cells grown in SILAC FLEX medium with the indicated carbon sources. All conditions were tested with the "t-test two sample assuming unequal variances" data analysis tool in Excel 2016. 
Cultured rat hippocampal neurons

\section{Mitochondrial diameter}

\begin{tabular}{|l|c|c|}
\hline & Glucose & bHB control \\
\hline Mean & 207.01 & 203.70 \\
\hline Variance & 3723.62 & 3302.71 \\
\hline Observations & 92 & 155 \\
\hline Hypothesized Mean Difference & 0 & \\
\hline df & 245 & \\
t Stat & 0.42815228 & \\
\hline$P(T<=t)$ one-tail & 0.3344582 & \\
\hline t Critical one-tail & 1.65109682 & \\
\hline$P(T<=t)$ two-tail & 0.6689164 & \\
\hline$t$ Critical two-tail & 1.96969392 & \\
\hline
\end{tabular}

Critical two-tail

\begin{tabular}{|l|c|c|}
\hline & & \\
\hline & & \\
\hline \multicolumn{2}{|c|}{ Average crista length per mitochondrial area } \\
\hline \multicolumn{2}{|c|}{ Glucose } & bHB control \\
\hline Mean & 9.22 & 10.19 \\
\hline Variance & 5.46 & 7.20 \\
\hline Observations & 68 & 67 \\
\hline Hypothesized Mean Difference & 0 & \\
\hline df & 133 & \\
\hline t Stat & -2.24381089 & \\
\hline$P(T<=t)$ one-tail & 0.01324884 & \\
\hline$t$ Critical one-tail & 1.65639124 & \\
\hline$P(T<=t)$ two-tail & 0.02649767 & \\
\hline$t$ Critical two-tail & 1.97796126 & \\
\hline
\end{tabular}
1.97796126

\section{Average crista length per mitochondrial length}

\begin{tabular}{|l|c|c|}
\hline & Glucose & bHB control \\
\hline Mean & 1.89 & 2.03 \\
\hline Variance & 0.62 & 0.91 \\
\hline Observations & 68 & 67 \\
\hline Hypothesized Mean Difference & 0 & \\
\hline df & 133 & \\
\hline t Stat & -0.93243059 & \\
\hline $\mathrm{P}(T<=t)$ one-tail & 0.17640182 & \\
\hline t Critical one-tail & 1.65639124 & \\
\hline $\mathrm{P}(T<=t)$ two-tail & 0.35280363 & \\
\hline $\mathrm{t}$ Critical two-tail & 1.97796126 & \\
\hline
\end{tabular}

\begin{tabular}{|c|c|}
\hline Glucose & bHB depol. \\
\hline 1.89 & 2.19 \\
0.62 & 0.55 \\
68 & 70 \\
0 & \\
136 & \\
\hline-2.27321412 & \\
0.0122903 & \\
1.65613499 & \\
\hline 0.02458059 & \\
\hline 1.97756078 & \\
\hline
\end{tabular}

\begin{tabular}{|c|c|}
\hline Glucose & bHB depol. \\
\hline 207.01 & 206.14 \\
\hline 3723.62 & 2787.02 \\
\hline 92 & 177 \\
0 & \\
\hline 267 & \\
0.12218917 & \\
\hline 0.45142056 & \\
\hline 1.6505806 & \\
0.90284112 & \\
\hline 1.96888862 & \\
\hline
\end{tabular}

\begin{tabular}{|c|c|}
\hline bHB control & bHB depol. \\
\hline 203.70 & 206.14 \\
3302.71 & 2787.02 \\
\hline 155 & 177 \\
0 & \\
330 & \\
-0.40291057 & \\
0.34363746 & \\
\hline 1.64948418 & \\
\hline 0.68727491 & \\
\hline 1.96717867 & \\
\hline
\end{tabular}

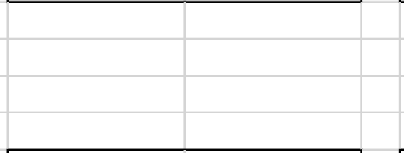

\begin{tabular}{|c|c|c|c|}
\hline Glucose & bHB depol. & bHB control & bHB depol. \\
\hline 9.22 & 11.06 & 10.19 & 11.06 \\
\hline 5.46 & 6.92 & 7.20 & 6.92 \\
\hline 68 & 70 & 67 & 70 \\
\hline 0 & & 0 & \\
\hline 136 & & 135 & \\
\hline-4.33068597 & & -1.9047093 & \\
\hline 1.4322E-05 & & 0.02947253 & \\
\hline 1.65613499 & & 1.65621913 & \\
\hline 2.8645E-05 & & 0.05894506 & \\
\hline 1.97756078 & & 1.97769228 & \\
\hline
\end{tabular}

Figure 46: Statistical tests for mitochondrial adaptation in cultivated rat hippocampal neurons. Neuronal cells were cultivated in the conditions indicated. Chemical depolarization of the neurons with $25 \mathrm{mM} \mathrm{KCl}$ for 4 hours. All conditions were tested with the "t-test two sample assuming unequal variances" data analysis tool in Excel 2016. 

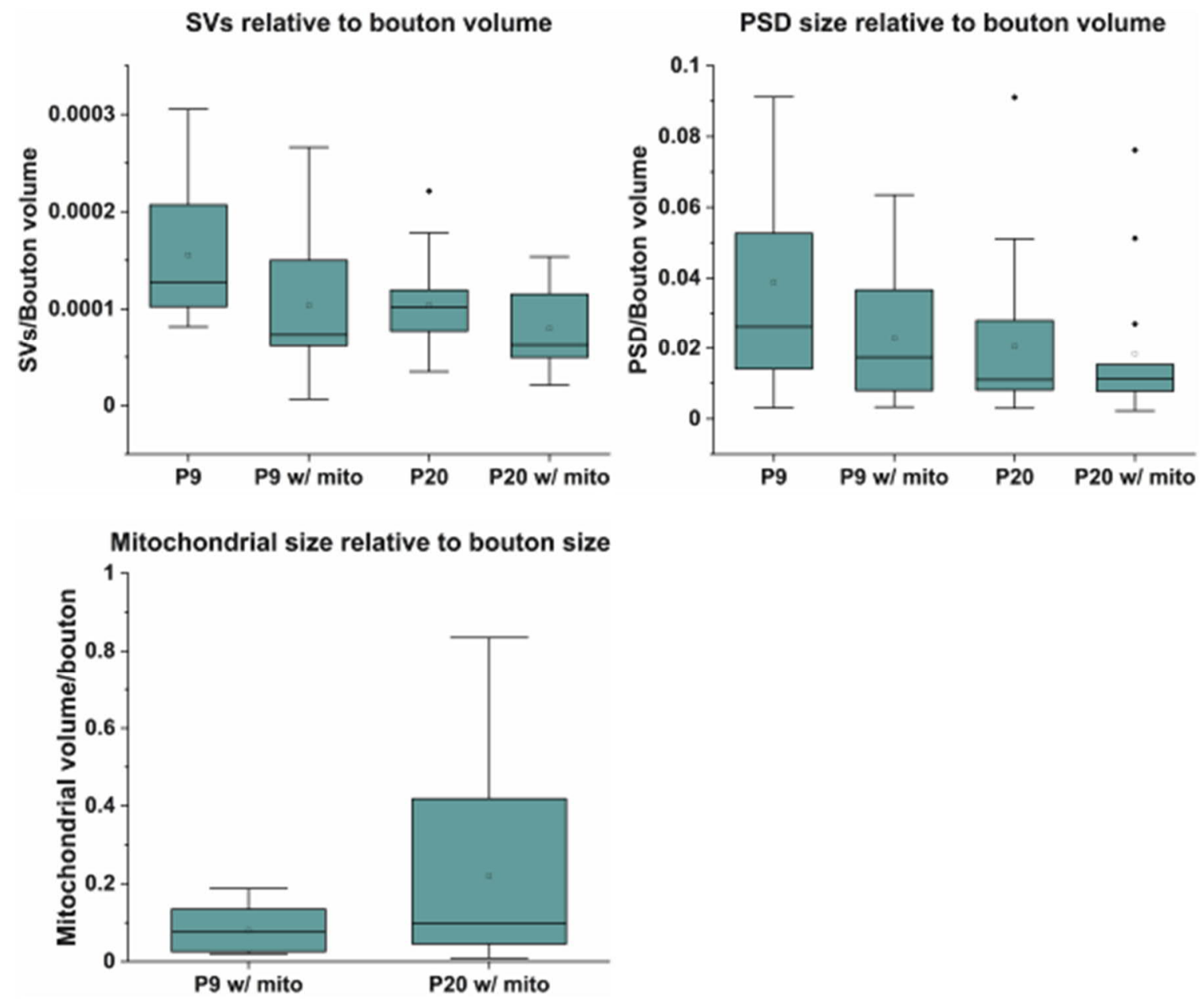

Figure 47: Box and whisker plots of features identified in FIB-SEM micrographs of the cochlear nucleus of a P9 and a P20 animal. Box plots show the ratio of the number of synaptic vesicles, the size of the PSD or the mitochondrial size to the volume of the presynaptic bouton. The boxes indicate $25^{\text {th }}$ to $75^{\text {th }}$ percentile. The small squares indicate mean. The horizontal lines indicate median. Whiskers indicate $S D$. 


\begin{tabular}{|c|c|c|c|c|c|c|}
\hline \multicolumn{7}{|l|}{ Bouton Volume } \\
\hline & $P 9$ & $P 9$ w/ mito & $P 9$ & $P 20$ & $P 9$ & $P 20 \mathrm{w} /$ mito \\
\hline Mean & 2384075.4 & 7439110.93 & 2384075.4 & 6315285.87 & 2384075 & 15170386.07 \\
\hline Variance & $1.589 \mathrm{E}+12$ & $2.7019 E+13$ & $1.5887 \mathrm{E}+12$ & $3.1091 \mathrm{E}+13$ & $1.6 \mathrm{E}+12$ & $1.58589 \mathrm{E}+14$ \\
\hline Observations & 15 & 15 & 15 & 15 & 15 & 15 \\
\hline Hypothesized Mean Difference & 0 & & 0 & & 0 & \\
\hline df & 28 & & 28 & & 28 & \\
\hline t Stat & -2.9971548 & & -2.1780313 & & -3.1775 & \\
\hline $\mathrm{P}(\mathrm{T}<=\mathrm{t})$ two-tail & 0.0064332 & & 0.03991065 & & 0.0042 & \\
\hline \multirow[t]{2}{*}{ t Critical two-tail } & 2.0686576 & & 2.06865761 & & 2.06866 & \\
\hline & $P 9 \mathrm{w} /$ mito & $P 20$ & $P 9 \mathrm{w} /$ mito & P20 w/ mito & $P 20$ & $P 20 \mathrm{w} /$ mito \\
\hline Mean & 7439110.9 & 6315285.87 & 7439110.93 & 15170386.1 & 6315286 & 15170386.07 \\
\hline Variance & $2.702 E+13$ & $3.1091 E+13$ & $2.7019 E+13$ & $1.5859 \mathrm{E}+14$ & $3.1 \mathrm{E}+13$ & $1.58589 \mathrm{E}+14$ \\
\hline Observations & 15 & 15 & 15 & 15 & 15 & 15 \\
\hline Hypothesized Mean Difference & 0 & & 0 & & 0 & \\
\hline df & 28 & & 28 & & 28 & \\
\hline t Stat & 0.5709767 & & -2.1978522 & & -2.4902 & \\
\hline $\mathrm{P}(\mathrm{T}<=\mathrm{t})$ two-tail & 0.5725701 & & 0.0363941 & & 0.01897 & \\
\hline t Critical two-tail & 2.0484071 & & 2.04840714 & & 2.04841 & \\
\hline \multicolumn{7}{|l|}{ PSD Volume } \\
\hline & $P 9$ & $P 9 \mathrm{w} /$ mito & $P 9$ & $P 20$ & $P 9$ & $P 20 \mathrm{w} /$ mito \\
\hline Mean & 76559.7 & 144771.653 & 76559.7 & 85090.8 & 76559.7 & 205277.2667 \\
\hline Variance & $3.91 \mathrm{E}+09$ & $2.0027 \mathrm{E}+10$ & 3909743480 & 4663070012 & $3.9 \mathrm{E}+09$ & 17762529438 \\
\hline Observations & 15 & 15 & 15 & 15 & 15 & 15 \\
\hline Hypothesized Mean Difference & 0 & & 0 & & 0 & \\
\hline df & 28 & & 28 & & 28 & \\
\hline t Stat & -1.4264455 & & -0.3161731 & & -2.8381 & \\
\hline $\mathrm{P}(\mathrm{T}<=\mathrm{t})$ two-tail & 0.1671738 & & 0.75472262 & & 0.00932 & \\
\hline \multirow[t]{2}{*}{ t Critical two-tail } & 2.0686576 & & 2.06865761 & & 2.06866 & \\
\hline & $P 9 \mathrm{w} /$ mito & $P 20$ & $P 9$ w/ mito & $P 20 \mathrm{w} /$ mito & $P 20$ & P20 w/ mito \\
\hline Mean & 144771.65 & 85090.8 & 144771.653 & 205277.267 & 85090.8 & 205277.2667 \\
\hline Variance & $2.003 E+10$ & 4663070012 & $2.0027 \mathrm{E}+10$ & $1.7763 \mathrm{E}+10$ & 4.7E+09 & 17762529438 \\
\hline Observations & 15 & 15 & 15 & 15 & 15 & 15 \\
\hline Hypothesized Mean Difference & 0 & & 0 & & 0 & \\
\hline df & 28 & & 28 & & 28 & \\
\hline t Stat & 1.4710232 & & -1.2054677 & & -3.1083 & \\
\hline $\mathrm{P}(\mathrm{T}<=\mathrm{t})$ two-tail & 0.1524349 & & 0.23811174 & & 0.00429 & \\
\hline t Critical two-tail & 2.0484071 & & 2.04840714 & & 2.04841 & \\
\hline \multicolumn{7}{|l|}{ Number of SVs } \\
\hline & $P 9$ & $P 9 \mathrm{w} /$ mito & $P 9$ & $P 20$ & $P 9$ & $P 20 \mathrm{w} /$ mito \\
\hline Mean & 325.6 & 738.533333 & 325.6 & 483.066667 & 325.6 & 846.2666667 \\
\hline Variance & 16965.378 & 401131.124 & 16965.3778 & 89214.2095 & 16965.4 & 122463.3524 \\
\hline Observations & 15 & 15 & 15 & 15 & 15 & 15 \\
\hline Hypothesized Mean Difference & 0 & & 0 & & 0 & \\
\hline $\mathrm{df}$ & 28 & & 28 & & 28 & \\
\hline t Stat & -2.0197012 & & -1.5624373 & & -4.4762 & \\
\hline $\mathrm{P}(\mathrm{T}<=\mathrm{t})$ two-tail & 0.0552136 & & 0.13184072 & & 0.00017 & \\
\hline \multirow[t]{2}{*}{ t Critical two-tail } & 2.0686576 & & 2.06865761 & & 2.06866 & \\
\hline & $P 9 \mathrm{w} /$ mito & $P 20$ & $P 9 \mathrm{w} /$ mito & $P 20 \mathrm{w} /$ mito & $P 20$ & $P 20 \mathrm{w} /$ mito \\
\hline Mean & 738.53333 & 483.066667 & 738.533333 & 846.266667 & 483.067 & 846.2666667 \\
\hline Variance & 401131.12 & 89214.2095 & 401131.124 & 122463.352 & 89214.2 & 122463.3524 \\
\hline Observations & 15 & 15 & 15 & 15 & 15 & 15 \\
\hline Hypothesized Mean Difference & 0 & & 0 & & 0 & \\
\hline $\mathrm{df}$ & 28 & & 28 & & 28 & \\
\hline t Stat & 1.4129567 & & -0.5766313 & & -3.0574 & \\
\hline $\mathrm{P}(\mathrm{T}<=\mathrm{t})$ two-tail & 0.1686889 & & 0.5687957 & & 0.00487 & \\
\hline t Critical two-tail & 2.0484071 & & 2.04840714 & & 2.04841 & \\
\hline \multicolumn{7}{|l|}{ Mitochondrial Volume } \\
\hline & $P 9 \mathrm{w} /$ mito & $P 20 \mathrm{w} /$ mito & & & & \\
\hline Mean & 386920.33 & 1939918.68 & & & & \\
\hline Variance & $1.922 \mathrm{E}+10$ & $4.8861 \mathrm{E}+12$ & & & & \\
\hline Observations & 15 & 15 & & & & \\
\hline Hypothesized Mean Difference & 0 & & & & & \\
\hline $\mathrm{df}$ & 28 & & & & & \\
\hline t Stat & -2.7157096 & & & & & \\
\hline $\mathrm{P}(\mathrm{T}<=\mathrm{t})$ two-tail & 0.0112024 & & & & & \\
\hline t Critical two-tail & 2.0484071 & & & & & \\
\hline
\end{tabular}


Figure 48: Statistical tests for structural features identified in FIB-SEM micrographs of a P9 and a P20 animal. All features of both conditions in both animals were compared and tested with the "t-test two sample assuming unequal variances" data analysis tool in Excel 2016. 


\section{Acknowledgements}

Finally, I want to thank all the people who accompanied and supported me throughout this thesis.

First, I want to thank Prof. Dr. Stefan Jakobs for giving me the opportunity to work on these two interesting projects in his group and for his guidance throughout the whole projects.

Next, I want to thank my two thesis advisors Prof. Dr. Peter Rehling and Prof. Dr. Reinhard Jahn for advices and helpful discussions that were a great help for this work.

I want to thank Prof. Dr. Stefan W Hell for giving me the opportunity to work in the Department for NanoBiophotonics.

I am especially grateful to Dr. Dietmar Riedel, Gudrun Heim and Dr. Dirk Wenzel for the fantastic training and teaching in sample preparation and transmission electron microscopy and the willingness for discussions and further the great work atmosphere in the facility. Both projects presented in this work would not have been possible without the support of them.

I want to thank Jasmin Hubrich, Ina Maria Herfort and Dr. Eugenio Fornasiero for the help to establish the rat hippocampal neuron cultures in our laboratory and of course Thomas Gundlach and Sascha Krause for the excellent help in the animal house and the constant provision of newborn rat puppies.

I want to thank Paola Agüí González and Prof. Dr. Silvio Rizzoli for the great help to establish and discuss the workflow for CLEM-SIMS and for providing NanoSIMS measurements and data analysis. This correlative project would not have been possible without them. Further, I want to thank Prof. Dr. Silvio Rizzoli for being a member of my examination board.

I want to thank Anika Hinze for providing the samples and the data for the in-vivo FIB-SEM analysis, Dr. Anna Steyer for FIB-SEM measurements and Prof. Dr. Carolin Wichmann for help and discussion of this work. Further, I want to thank Prof. Dr. Carolin Wichmann for being a member of my examination board.

Additionally, I want to thank Prof. Dr. Tiago Outeiro for being a member of my examination board.

I want to thank Rita Schmitz-Salue, Tanja Koenen, Sylvia Löbermann, Nicole Molitor, and Ellen Rothermel for excellent technical assistance and for keeping the lab running.

I want to thank Dr. Julia Preobraschenski, Dr. Eugenio Fornasiero and Dr. Marcelo Ganzella for the help and discussion of all neurobiology related questions of mine. 
I would also like to thank Dr. Nickels Jensen, Dr. Julia Preobraschenski, Dr. Daniel Jans, Dr. Stefan Stoldt and Jaydev Jethwa, for proofreading of this thesis.

I am also very thankful for all my colleagues in the Department of NanoBiophotonics who were all a great help in professional and personal matters during this work. Especially I want to thank Dr. Nickels Jensen, Dr. Daniel Jans and Dr. Stefan Stoldt for training and help with fluorescence microscopy.

Last, I want to thank my friends and my family for the immense love and support throughout the work. 